\begin{tabular}{|c|c|}
\hline JUN 171996 ENGINEERING data transmittal & 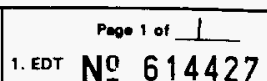 \\
\hline
\end{tabular}

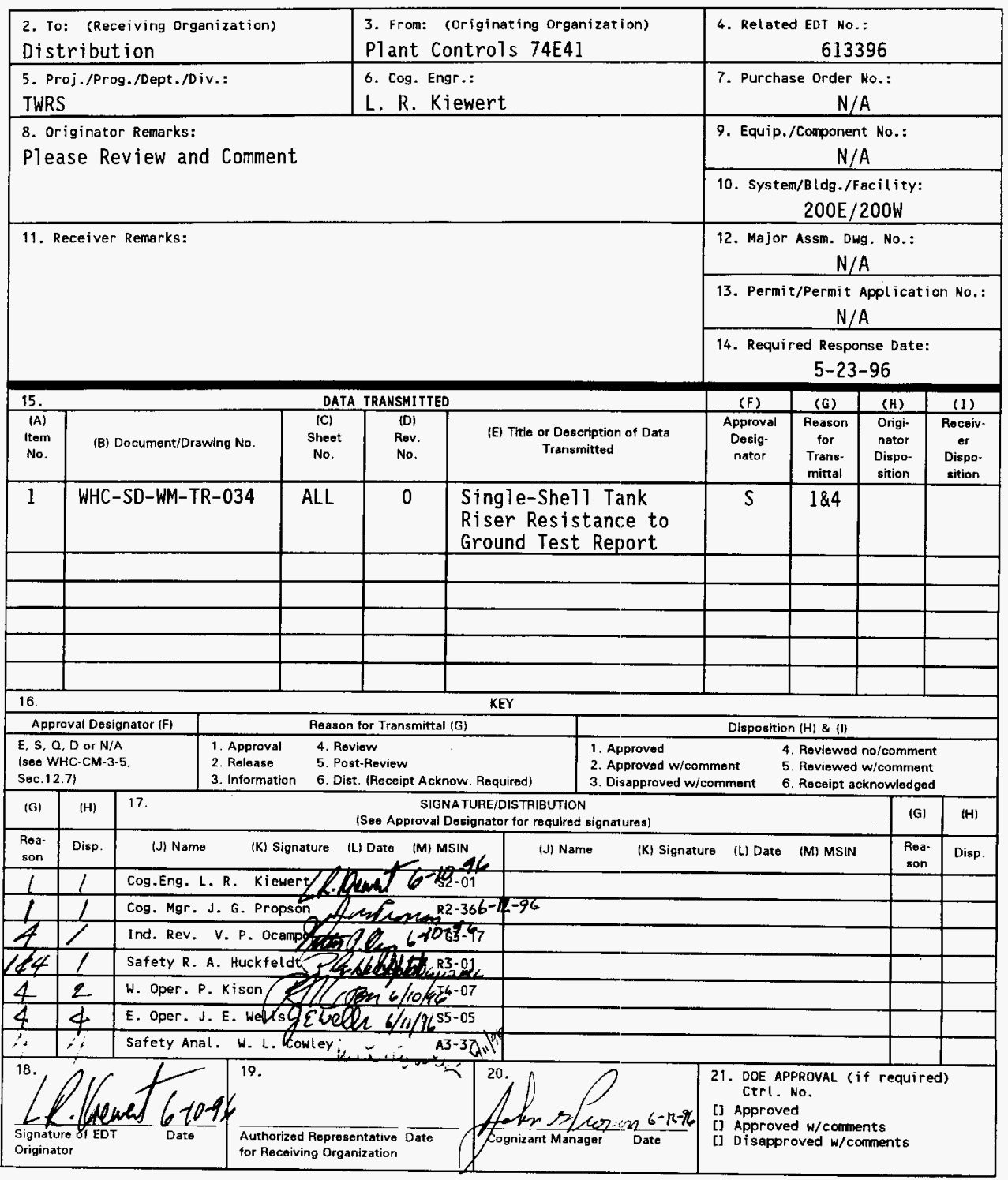

BD-7400-172-2 (04/94) GEF097 


\section{SINGLE-SHELL TANK RISER RESISTANCE TO GROUND TEST REPORT}

\section{L.R. Kiewert}

WHC, Richland, WA 99352

U.S. Department of Energy Contract DE-AC06-87RL10930

$\begin{array}{lll}\text { EDT: } & 614427 & \text { UC: } 721 \\ \text { Org Code: } & 74 E 41 & \text { Charge Code: N2148 } \\ \text { B\&R Code: } & \text { EW3120072 } & \text { Total Pages: } 197\end{array}$

Key Words: RISER RESISTANCE MEASUREMENTS

Abstract: This Test Report provides the results from Single-Shell Tank Riser to Earth Measurements which were conducted by engineering as a step towards providing closure for the Lightning Hazard Issue.

TRADEMARK DISCLAIMER. Reference herein to any specific commercial product, process, or service by trade
name, trademark, manufacturer, or otherwise, does not necessarily constitute or imply its endorsement,
recommendation, or favoring by the United states Government or any agency thereof or its contractors or recommendat ion,

Printed in the United States of America. To obtain copies of this doctment, contact: WHC/BCS Document Control Services, P.O. Box 1970, Mailstop H6-08, Richland WA 99352, Phone (509) 372-2420; Fax (509) 376-4989.
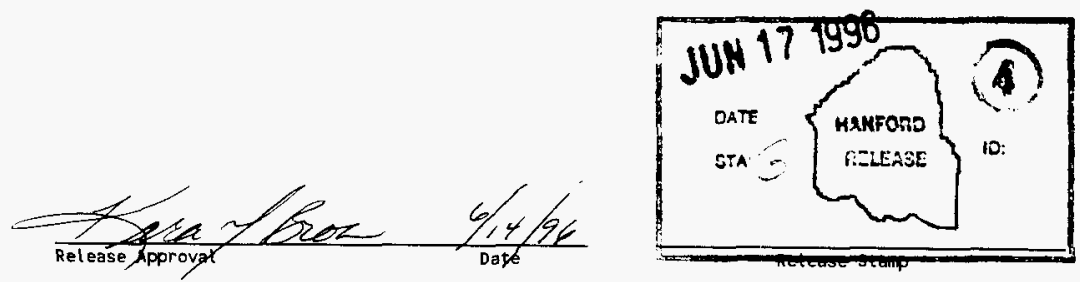

\section{Approved for Public Release}


WHC-SD-WM-TR-034, Rev. 0

TABLE OF CONTENTS

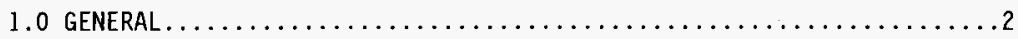

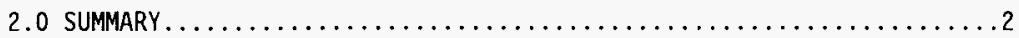

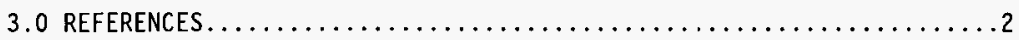

4.0 DATA INTERPRETATION

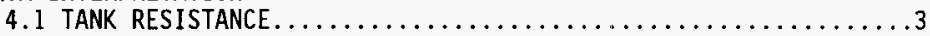

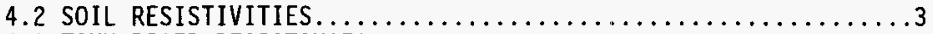

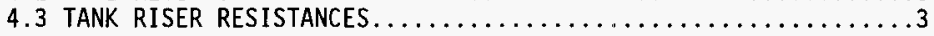

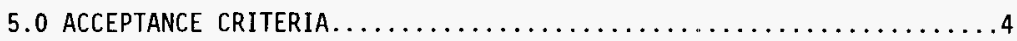

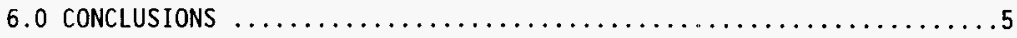

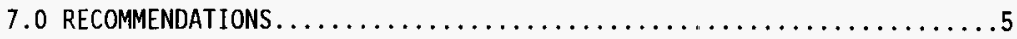

APPENDIX A

DATA COLLECTION METHODS ..........................

SOIL RESISTIVITY TEST ILLUSTRATION. ...................

SINGLE-SHELL TANK RISER RESISTANCE MEASUREMENTS ............. A-4

APPENDIX B

TANK FARM SOIL RESISTIVITY DATA SHEET $\ldots \ldots \ldots \ldots \ldots \ldots \ldots \ldots$.

APPENDIX C

RISER RESISTANCE DATA SHEETS...............

APPENDIX D

TYPICAL GROUNDING AND BONDING SCHEME............ D-1 thru D-2 
SINGLE-SHELL TANK RISER

RESISTANCE TO EARTH GROUND TEST REPORT

\subsection{GENERAL}

Between the dates of March 23, 1996 and May 9, 1996 measurements were made on all accessible risers on the 149 single shell tanks per Test Plan WHC-SD-WMTP-433. These measurements determined the resistance to earth which is of interest in evaluating the path to ground of a lightning strike through a riser. The techniques used to measure the riser resistivity are described in Appendix A. Within this report an acceptance criteria is established and riser measurements are compared to that criteria, conclusions are drawn and recommendations are made. Data sheets for each tanks measurements are found in Appendices B \& C.

\subsection{SUMMARY}

The resistance to ground of accessible risers, attached equipment and other selected structures associated with the single shell tanks were measured. Additionally any metal structure above a tank was measured for comparison with the values for the tank risers.

A qualified acceptance criteria of 5 ohms maximum is assigned to the tank risers.

1094 Risers were measured on 149 tanks

213 Risers did not meet the acceptance criteria

65 Tanks - all risers met acceptance criteria

Soil resistivities were measured for each single she 11 tank farm location.... $A, A X, B, B X, B Y, C, S, S X, T, T X, T Y, U$ and are recorded on a data sheet in Appendix B. Values varied between 15K - 50K ohm - centimeters.

\subsection{REFERENCES}

IEEE STD 142-1991, "Grounding of Industrial And Commercial Power Systems."

IEEE STD 81-1983, "Guide For Measuring Earth Resistivity, Ground Impedance, And Earth Surface Potentials of A Ground System."

NFPA 780-95, "Standard For Installation of Lightning Protection Systems"

WHC-SD-RE-TI-053, "Riser Configuration Document For Single-She11 Waste Tanks."

WHC-SD-WM-TP-433, "Single Shell Tank Riser Resistance to Ground Test Plan" 
WHC-SD-WM-TR-034, Rev. 0

\subsection{DATA INTERPRETATION}

SOIL RESISTIVITIES - varied between 15K and 50K ohm centimeters and were fairly consistent from tank farm to tank farm - the lower readings were in the vicinity of the 200 East Farms and the higher readings those found around the 200 West Farms. The soil resistivity appeared to have had little bearing on the results of the ground resistance measurements for most tank farms.

TANK RESISTANCE TO EARTH - The tanks exhibited low values of grounding resistance - (less than $1 \mathrm{ohm}$ in many cases) representative of the large area of reinforced concrete in contact with the soil. The tank ground resistance is taken to be the average value of acceptable riser resistances measured and is meaningful for determining the resistance of the riser to tank connection. Typical1y the acceptable riser resistances of a tank were measured in a narrow range with the difference attributable to the geometry of the current and voltage pins. In most cases the resistivities for different tanks within a given tank farm were similar being representative of the piping in the ground and again the location of the current and potential pins. Whenever possible the pins were located outside the tank farm fence in an undeveloped area. This was not always possible and the result in a few cases was exceptionally low readings that generally corresponded to a current path that included parallel piping and other tanks. The exact magnitude of the resistance measured is not as important as is the comparison of the different riser readings for a tank.

TANK RISER RESISTANCE TO EARTH - AlI risers and their attachment(s) were measured separately and all measurements were made to bare metal to insure no surface resistance. Results indicated that original construction tank risers with some exceptions are well connected electrically to the tanks. In most cases the acceptable riser resistance to ground for a given tank was less than 4 ohms and varied only a few hundredths of an ohm regardless of whether the riser was connected to the waste.

The original riser drawings for each tank were researched and compared with the "As Built" configuration. There are a total of 319 risers added after original construction. These risers are flagged as indicated on the Legend for the data sheets. One category of these risers was installed through corebores and metal to metal contact is incidental rather than constructed. The other category of these added risers is single or multiple risers welded to the blank flange cover of a large (usually $42^{\prime \prime)}$ unused riser. The riser connection is bolted and is typically underground.

Total risers measured..... 1094

Total original construction risers measured . . . 775

Total added risers measured....319

In most cases these added risers measured a higher resistance than the original construction risers.

The resistance between risers and attachments varied from no resistance to several thousand ohms (this is a multibolt connection with a gasket in between). It is to be noted that those risers which had recently been 
WHC-SD-WM-TR-034, Rev. 0

regasketed or otherwise reworked showed little or no resistance while the resistance exhibited by others can be attributed to corrosion buildup underneath the boltheads and nuts. The importance of this measurement is that it points out the sensitivity of the instrumentation and indicates that the riser attachment to the reinforcing steel of the tank is extremely secure where the resistivity of the riser is within a few hundredths of an ohm of the measured value on the other risers. Note that this conclusion is corraborated with photographs of construction steel details from 1947 showing rebar to riser connection.

Generally the electrical boxes, receptacles, and ground cables indicated ground resistance values that were considerably larger than the riser resistance values - another indication of the quality of the original tank riser connection to ground. The data acquisition system (TMACS) field stations are connected to most of the various thermocouple trees and to the Enraf level guages with a PVC coated rigid steel conduit - this connection provides an excellent insulated current path between the TMACS box and the tank sensor, especially the thermocouple probe. In some cases this connection is made via an underground conduit junction box with the conduits bonded together in the box. In a few cases the thermocouple probe was isolated by temporarily removing the bonding jumper and confirming that that the measured value was attributed to the riser or probe.

The thermocouple probes in some cases exhibited lower resistance than the riser - the result of high conductivity of the waste surrounding the probe coupled with the probe to flange resistance. These are designated as "Grounded Through Waste" (GTW) on the data sheets.

\subsection{ACCEPTANCE CRITERIA}

The criteria from IEEE 142-1991 of "1 to 5 ohms ground resistance for large industrial installations" provides a good fit to the data collected. All tanks that were measured exhibited resistances to ground of less than 5 ohms, and riser resistances to ground varied from less than 1 ohm to $>20 \mathrm{~K}$ ohms. The tank resistance to ground is determined by averaging the resistance values of the original construction risers which fall into a narrow band with a spread of about $10 \%$ to $20 \%$.

The general acceptance criteria for riser resistance values is 5 ohms - where the riser measurement is more than 5 ohms, the riser is flagged "Ungrounded Riser" (UGR).

Where the tank can be considered hazardous (Watchlist Tanks which contain ignitable gases or organic materials), the $5 \mathrm{ohm}$ criteria does not apply - The acceptable riser to ground resistance value is only apparent after reviewing the data for that tank. In this case acceptable readings fall into a narrow range around tank resistance value. Riser resistance values less than 5 ohms which may not be considered acceptable to safely conduct a lightning strike to ground are not flagged on the data sheets.

Although the NEC considers that a grounding resistance of 25 ohms or less is acceptable for a single made electrode - that criteria is not applicable in this case in that the tank resistances in every case has been demonstrated to 
WHC-SD-WM-TR-034, Rev. 0

be less than 5 ohms and any resistance greater than the tank resistance represents the electrode resistance (riser to tank connection).

\subsection{CONCLUSIONS}

Acceptable riser resistance values were less than 5 ohms. Using the acceptance criteria there are 84 tanks with risers which are unacceptable. Risers which do not meet the acceptance criteria are flagged. It is to be noted that most of the risers on the sixteen 200 series tanks (50,000 gallon) have high readings which indicate that the method of installing or attaching those risers to the tank was different than on the larger tanks.

A11 risers which are indicated as added after original construction should be considered questionably grounded (even when not flagged UGR).

Bonding/grounding will need to be considered for these risers when the tank is considered hazardous (Watch List Hydrogen or Organic) regardless of the measured riser resistance value. Supplementary grounding will be effective only when the grounding point chosen is close to the tank resistance value.

The results of the resistance measurements indicate that thermocouple trees present the most credible entrance path for a lightning strike into a tank because of the insulated rigid steel conduit connection to the TMACS field station. This connection multiplies the surface area over which a lightning strike could be channeled into the tank. There are 21 tanks with risers which contain thermocouple trees which have been shown to be grounded through the waste (flagged as GTW) - supplementary grounding/bonding will not create a path of less resistance in this case and it will be necessary to shield or isolate the thermocouple tree from a direct lightning strike. Additionaliy the conduit connection to TMACS will need to be isolated to prevent an indirect strike on the TMACS field station or connecting conduits from being conducted into the tank(s) waste.

Unacceptable original risers requiring review.....71 (200Series...56) Added risers requiring review........319

\subsection{RECOMMENDATIONS}

There are a number of remediation steps which can be taken to acceptably ground a tank riser/attachments to prevent arcing during an electrical short circuit or lessen the vulnerability of a tank riser from a lightning strike. Solutions initiated for lightning protection should be applied using a graded approach concentrated on those tanks which can be considered hazardous and those risers which are not under the zone of protection of another structure. Solutions for unacceptable risers utilized for grounding electrical equipment are required regardless of tank classification.

A properly grounded and bonded riser will efficiently conduct a lightning stroke to ground thus eliminating in most cases that riser/attachment as a potential source of ignition for flammable tank contents when subjected to a lightning strike. Likewise a riser which is shielded from direct lightning strikes and isolated from indirect lightning strikes can be eliminated as a 
WHC-SD-WM-TR-034, Rev. 0

hazardous target. Schemes for providing the remediation are depicted in Appendix D.

LIGHTNING ANALYSIS - Utilize NFPA 780 "Standard for the Installation of Lightning Protection Systems" zone of protection concepts for selecting risers which need grounding or other protection. This process will also selectively remove other risers from consideration as a lightning target. Riser attachments which protrude into the waste should be carefully assessed for the effectiveness of shielding or bonding.

BONDING - The results of the testing indicate that many risers are an excellent electrical ground, however the attachments may or may not be. A bonding strap (See appendix D-1) bolted to the side of the riser and to the attachment flange(s) would permanently eliminate the resistance thus providing an excellent ground path for a lightning strike. A bonding link should be utilized on all bolted joints on risers that are considered lightning targets by virtue of their physical configuration.

GROUNDING - Those risers which are indicated as ungrounded and which are used as a ground need to have a permanent low resistance ground provided in accordance with NEC Article 250 Part $H$ and K. In some cases the data sheets provide assistance for locating an alternate ground point (I.E. - ground pad adjacent to BX106).

In general those risers found to be ungrounded on hazardous tanks should be grounded for lightning protection even if they can be shown to be in the zone of protection of an another structure.

Where thermocouple trees are indicated as grounded through the waste (GTW) a parallel ground path would not provide much help in shunting off a lightning strike. If the tank is considered Watch List Hydrogen or Organic, a local air terminal (See appendix D-2) will need to be provided to intercept a direct lightning strike unless the thermocouple tree is determined to be in the zone of protection of another structure.

SHIELDING - Shielding can be utilized for lightning protection in certain cases. For example Manual Tapes can be fitted with a steel box which would surround the electrically isolated top section. The box would act as a Faraday Cage and shunt the lightning strike around the isolated tape reel to a grounded riser.

ISOLATION - Provide isolation between the TMACS field station and hazardous tanks to prevent coupling energy from an indirect lightning strike on a TMACS cabinet or conduit into the tank through a thermocouple probe or other tank sensor Removing the PVC jacket from a buried section of the conduit would serve to provide a ground path. 
WHC-SD-WM-TR-034, REV. 0

APPENDIX A

DATA COLLECTION METHODS

\section{SOIL RESISTIVITY MEASUREMENTS}

Soil resistivity was measured for each general tank farm area where ground resistance measurements were taken in order to account for any significant differences in the resistance to earth data from tank farm to tank farm. The Wenner Four Electrode Method (ASTM Standard G57-78-86) is used to measure resistivity at various depths at tank farm locations. This procedure involves the use of four electrodes which are driven in the soil along a straight line at equal spacing. The spacing determines the depth at which the soil is evaluated. Electrical current is circulated through the two outside electrodes while the resultant voltage drop is measured across the two inside electrodes. Using Ohms Law, the resistance is calculated by dividing the measured voltage drop by the applied current to yield resistance. Appendix A3 illustrates this procedure. Once the resistance of the soil at a particular depth is determined, it is converted to resistivity by multiplying the value by a constant (191.5) and the electrode spacing(depth) in feet.

RHO (ohm-cm) $=\mathrm{R} \times \mathrm{d} \times 191.5$

$R=$ measured resistance, $d=$ distance between electrodes in feet. 191.5 = constant derived from $2 \times 3.14 \times 12$ in. $/ \mathrm{ft} . \times 2.54 \mathrm{~cm} . / \mathrm{in}$.

The calculated value is the resistivity in ohm-centimeters. The soil pins are pushed into the earth by hand extending below the surface approximately 6 inches. The spacing between the pins is 10 feet for these tests. Four pins are used and they are connected to the resistance meter using insulated test leads. The actual resistance is read off the digital display and converted to resistivity in ohm- $\mathrm{cm}$. 


\section{RESISTANCE TO EARTH MEASUREMENTS}

The measurement of riser to ground resistances was conducted with a low voltage test instrument which was attached by test leads to steel pins which are driven into the ground approximately $12^{\prime \prime}$ so that the safety of underground structures beneath the pins is insured. Test current of 10 ma maximum was circulated from the riser through the tank structure, through the ground to the current electrode. The connection at the riser or other structure to be measured was made with an alligator clip or other tension clip to assure a good connection. The method of measuring riser to ground resistance was the "Fall of Potential Method" - This procedure was used for all measurements taken while conducting the test.

The fall of potential method uses a current electrode(pin) and a potential electrode(pin) arranged in a straight line away from the structure under test as indicated in Appendix A-4. Using this arrangement, current is introduced into the earth using the current electrode and the resulting change in potential between the structure and the potential electrode is measured. Resistance is plotted as a function of the distance between the structure and the potential electrode which is moved away from the structure being measured until the resistance levels out.

In practice the test is conducted in the same manner as the soil resistivity test using the same meter to supply a low voltage alternating current between the structure under test and the two pins that are driven in the ground some distance away. Typically the current pin is 10cated 500-1000' from the structure, while the potential pin is located approximately $62 \%$ of the distance to the current pin. In all cases sufficient data is gathered by moving the potential pin in a straight line towards the current pin until the resistance remains relatively constant.

When difficulties were encountered to obtain measurements for a specific tank it was necessary to use a reference ground or dead earth to obtain measurements. A typical reference ground could be buried piping. The Biddle Earth Tester was used for all testing. This unit is a 4 terminal direct digital readout ohmmeter. The tester generates a low voltage (50VAC) $135 \mathrm{~Hz}$ current (0-10ma) between the current posts. The input to the detector section of the Tester is filtered and not affected by stray A.C. or D.C. currents and converts the voltage measurements directiy into resistance. 


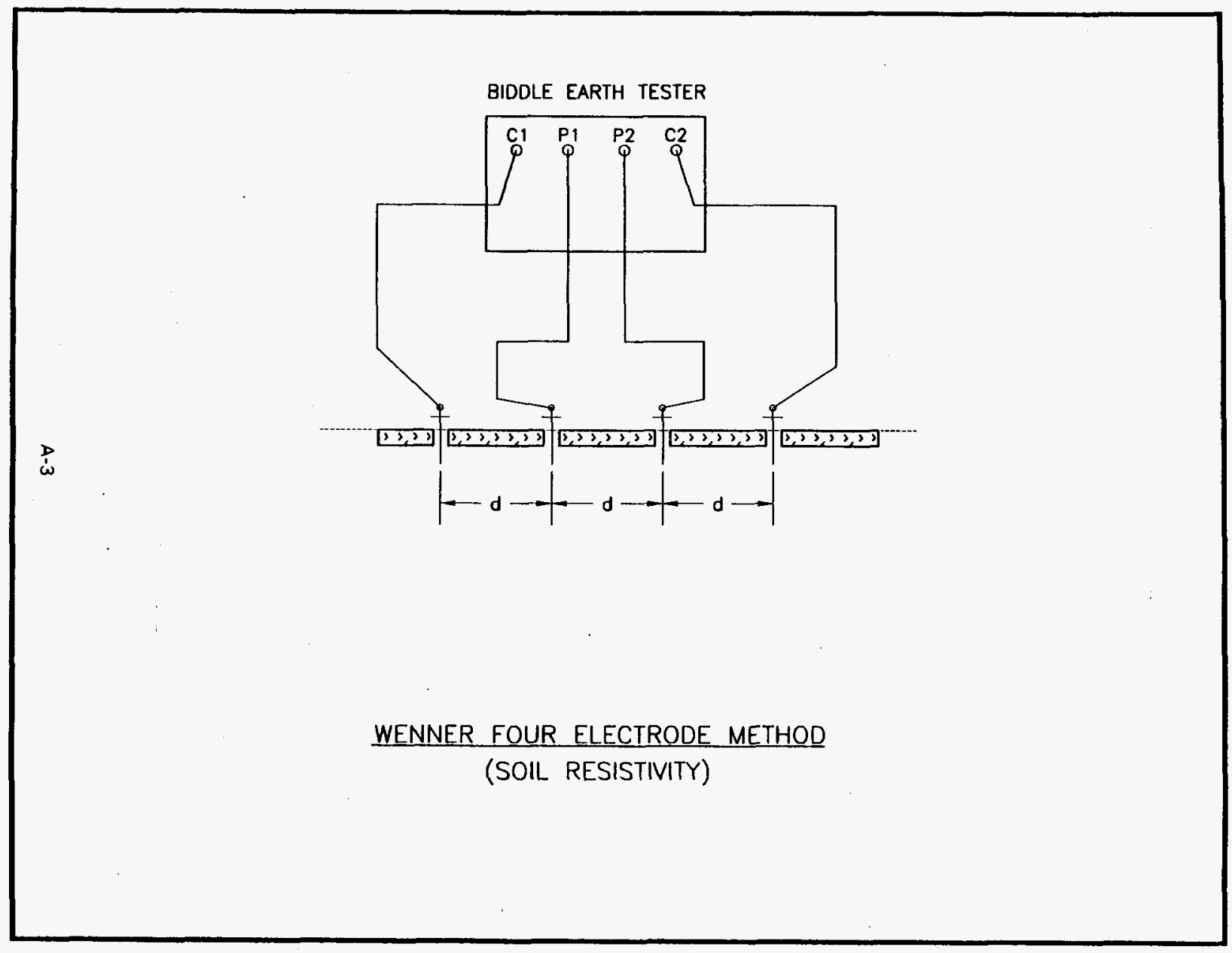


WHC-SD-WM-TR-034, Rev. 0

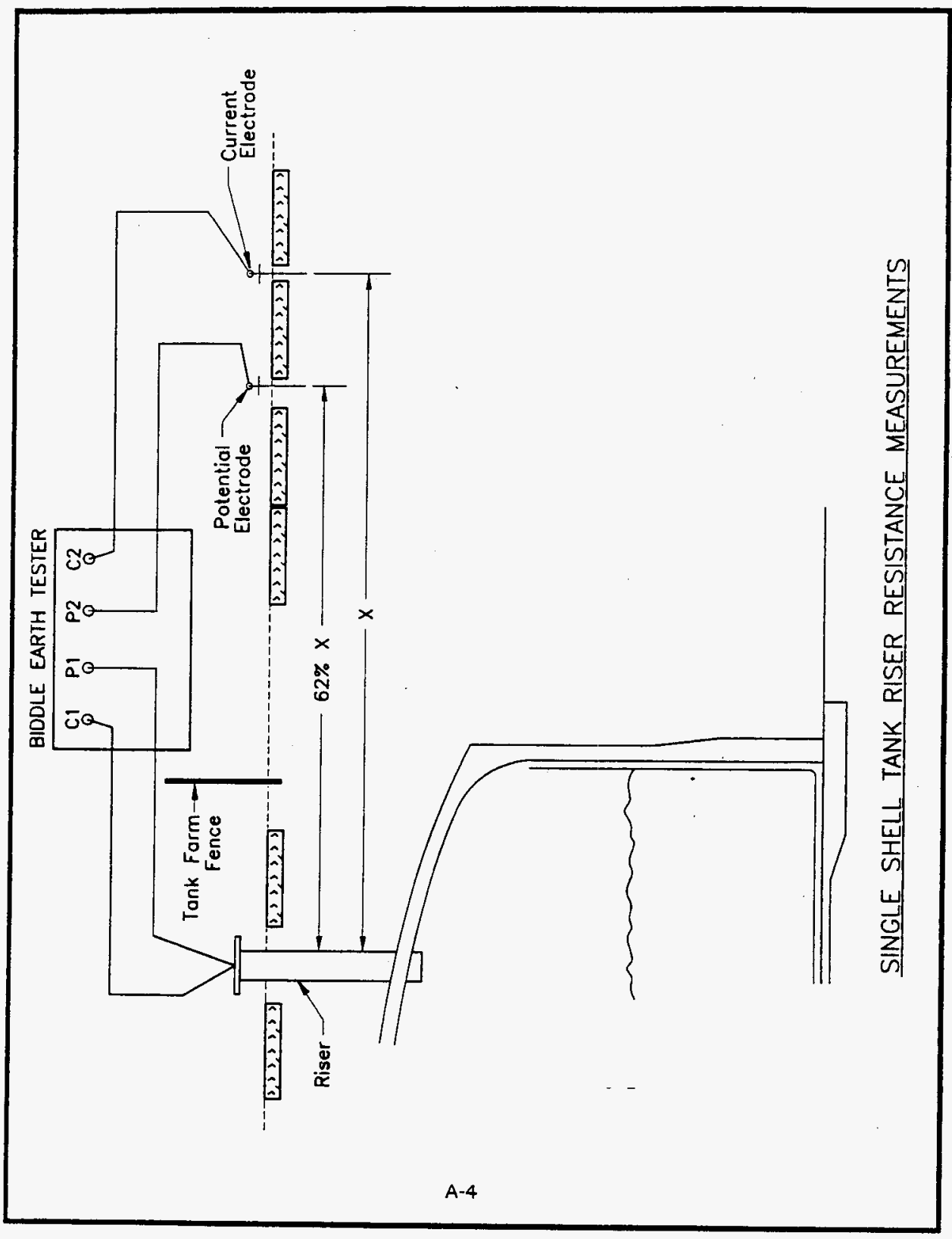




\section{TANK FARM SOIL RESISTIVITY DATA SHEET}

\begin{tabular}{|c|c|l|c|}
\hline TANK FARM & DATE & \multicolumn{1}{|c|}{ LOCATION } & RESISTIVITY \\
\hline \hline $241-\mathrm{A}$ & $5 / 9 / 96$ & SOUTH OUTSIDE & $15.7 \mathrm{~K} \Omega$ \\
\hline $241-\mathrm{AX}$ & $4 / 18 / 96$ & $\begin{array}{l}\text { NORTHEAST CORNER OUTSIDE } \\
\text { NORTHEAST CORNER INSIDE }\end{array}$ & $\begin{array}{l}21.3 \mathrm{~K} \Omega \\
35.7 \mathrm{~K} \Omega\end{array}$ \\
\hline $241-\mathrm{B}$ & $5 / 1 / 96$ & $\begin{array}{l}\text { NORTH SIDE OUTSIDE } \\
\text { NORTHEAST CORNER OUTSIDE }\end{array}$ & $\begin{array}{l}14.7 \mathrm{~K} \Omega \\
28.5 \mathrm{~K} \Omega\end{array}$ \\
\hline $241-\mathrm{BX}$ & $4 / 9 / 96$ & $\begin{array}{l}\text { WEST SIDE OUTSIDE } \\
\text { WEST SIDE OUTSIDE }\end{array}$ & $\begin{array}{l}30.8 \mathrm{~K} \Omega \\
21.8 \mathrm{~K} \Omega\end{array}$ \\
\hline $241-\mathrm{BY}$ & $4 / 29 / 96$ & WEST SIDE OUTSIDE & $28.7 \mathrm{~K} \Omega$ \\
\hline $241-\mathrm{C}$ & $4 / 16 / 96$ & $\begin{array}{l}\text { SOUTHWEST CORNER INSIDE } \\
\text { SOUTHWEST CORNER INSIDE }\end{array}$ & $\begin{array}{l}25.5 \mathrm{~K} \Omega \\
19.4 \mathrm{~K} \Omega\end{array}$ \\
\hline $241-\mathrm{S}$ & $3 / 30 / 96$ & $\begin{array}{l}\text { WEST SIDE OUTSIDE } \\
\text { SOUTH SIDE INSIDE }\end{array}$ & $\begin{array}{l}37.6 \mathrm{~K} \Omega \\
23.6 \mathrm{~K} \Omega\end{array}$ \\
\hline $241-S X$ & $3 / 30 / 96$ & $\begin{array}{l}\text { SOUTHWEST CORNER OUTSIDE } \\
\text { NORTHWEST CORNER OUTSIDE }\end{array}$ & $\begin{array}{l}34.7 \mathrm{~K} \Omega \\
24.2 \mathrm{~K} \Omega\end{array}$ \\
\hline $241-T$ & $3 / 25 / 96$ & $\begin{array}{l}\text { WEST SIDE OUTSIDE } \\
\text { NORTHEAST CORNER OUTSIDE }\end{array}$ & $\begin{array}{l}34.9 \mathrm{~K} \Omega \\
28.9 \mathrm{~K} \Omega\end{array}$ \\
\hline $241-T X$ & $3 / 28 / 96$ & $\begin{array}{l}\text { WEST SIDE OUTSIDE } \\
\text { EAST SIDE OUTSIDE }\end{array}$ & $\begin{array}{l}35.4 \mathrm{~K} \Omega \\
16.7 \mathrm{~K} \Omega\end{array}$ \\
\hline $241-T \mathrm{~T}$ & $3 / 23 / 96$ & $\begin{array}{l}\text { WEST SIDE OUTSIDE } \\
\text { EAST SIDE OUTSIDE }\end{array}$ & $\begin{array}{l}47.2 \mathrm{~K} \Omega \\
15.5 \mathrm{~K} \Omega\end{array}$ \\
\hline $241-\mathrm{U}$ & $4 / 11 / 96$ & $\begin{array}{l}\text { WEST SIDE OUTSIDE } \\
\text { NORTH SIDE OUTSIDE }\end{array}$ & $\begin{array}{l}50.4 \mathrm{~K} \Omega \\
26.6 \mathrm{~K} \Omega\end{array}$ \\
\hline
\end{tabular}

TEST EQUIP \# 785-77-47-001

CALIB DUE DATE $3-12-97$ 



\section{RISER RESISTANCE TO GROUND DATA SHEETS}

LEGEND

$\Sigma$ RISER NUMBER

(8) - RISER ADDED AFTER ORIGINAL CONSTRUCTION

$\ulcorner$ RISER NUMBER

TOA - 4", 12" OR 18" RISER ATTACHED TO 42" RISER BELOW GRADE

U.G.R. - UNGROUNDED RISER

G.T.W. - RISER/PROBE GROUNDED THROUGH WASTE 


\section{RISER RESISTANCE DATA SHEET}

TANK $A-101$
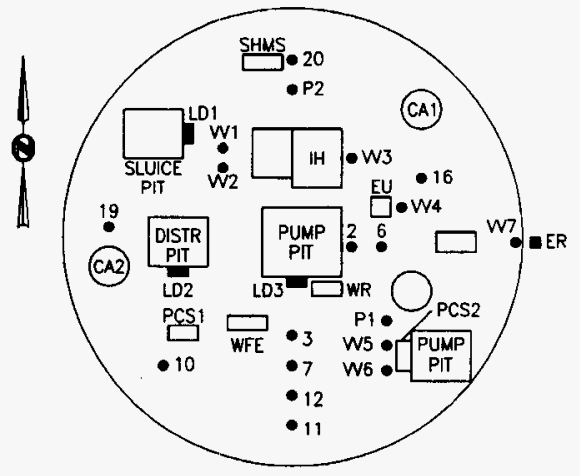

\begin{tabular}{|c|c|c|c|c|}
\hline \multirow{2}{*}{ RISER } & \multirow{2}{*}{ DESCRIPTION } & \multicolumn{2}{|c|}{ RESISTANCE(OHMS) } & \multirow{2}{*}{ COMMENTS } \\
\hline & & RISER & TOP & \\
\hline 2 & 8" THERMOCOUPLE & .06 & .06 & \\
\hline 3 & 8" DRYWELL & .05 & .23 & \\
\hline 6 & $4^{\prime \prime}$ ENRAF & .05 & .10 & \\
\hline 7 & 4" CRUST BRKR & .09 & .09 & \\
\hline 10 & 4" SPARE & .07 & .09 & \\
\hline 11 & 4" WASTE LINE & 2.83 & 2.83 & \\
\hline (12) & 4" TEMP PROBE & .09 & .09 & \\
\hline (16) & OBSV PORT & .28 & .29 & \\
\hline (19) & 4" SPARE & 43.8 & 43.8 & U.G.R. \\
\hline \multirow[t]{2}{*}{ (20) } & 4" BREATHER FLTR & .22 & .23 & \\
\hline & SHMS CABINET & & .16 & \\
\hline P2 & 1" PIPE & & 33.2 & \\
\hline $\mathbb{H H}$ & INSTRUMENT HOUSE & & .37 & \\
\hline CA1 & 4' CAISSON & & .62 & \\
\hline W3 & VALVE HANDLE & & .67 & \\
\hline \multicolumn{2}{|c|}{ CONTINUED NEXT SHEET } & \multicolumn{2}{|c|}{$\begin{array}{l}\text { TEST EQUIP \# } \\
\text { CALIB DUE DA }\end{array}$} & $\begin{array}{l}785-77-47-001 \\
3-12-97\end{array}$ \\
\hline
\end{tabular}




\section{RISER RESISTANCE DATA SHEET}

TANK A-101 CONTINUED

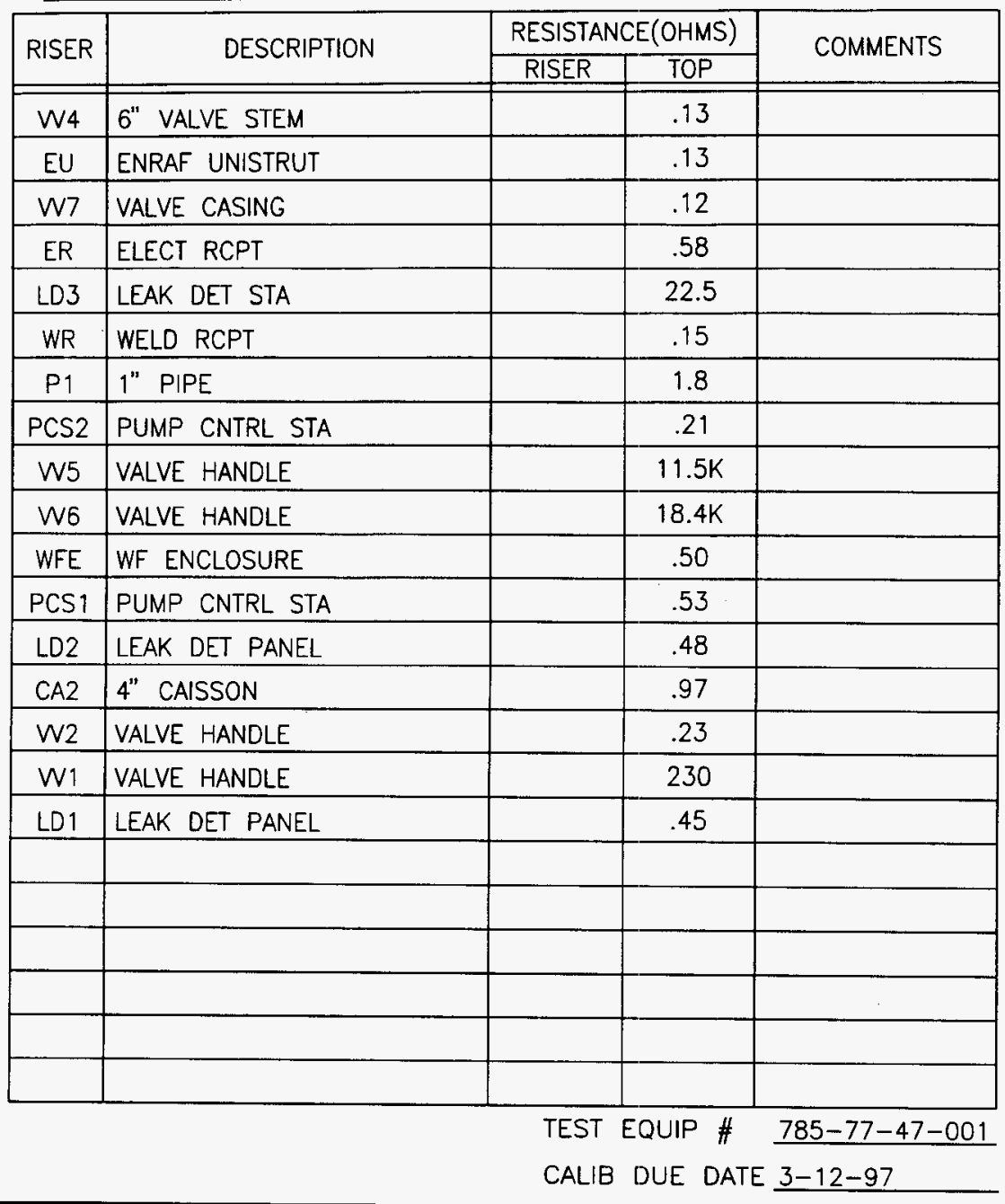




\section{RISER RESISTANCE DATA SHEET}

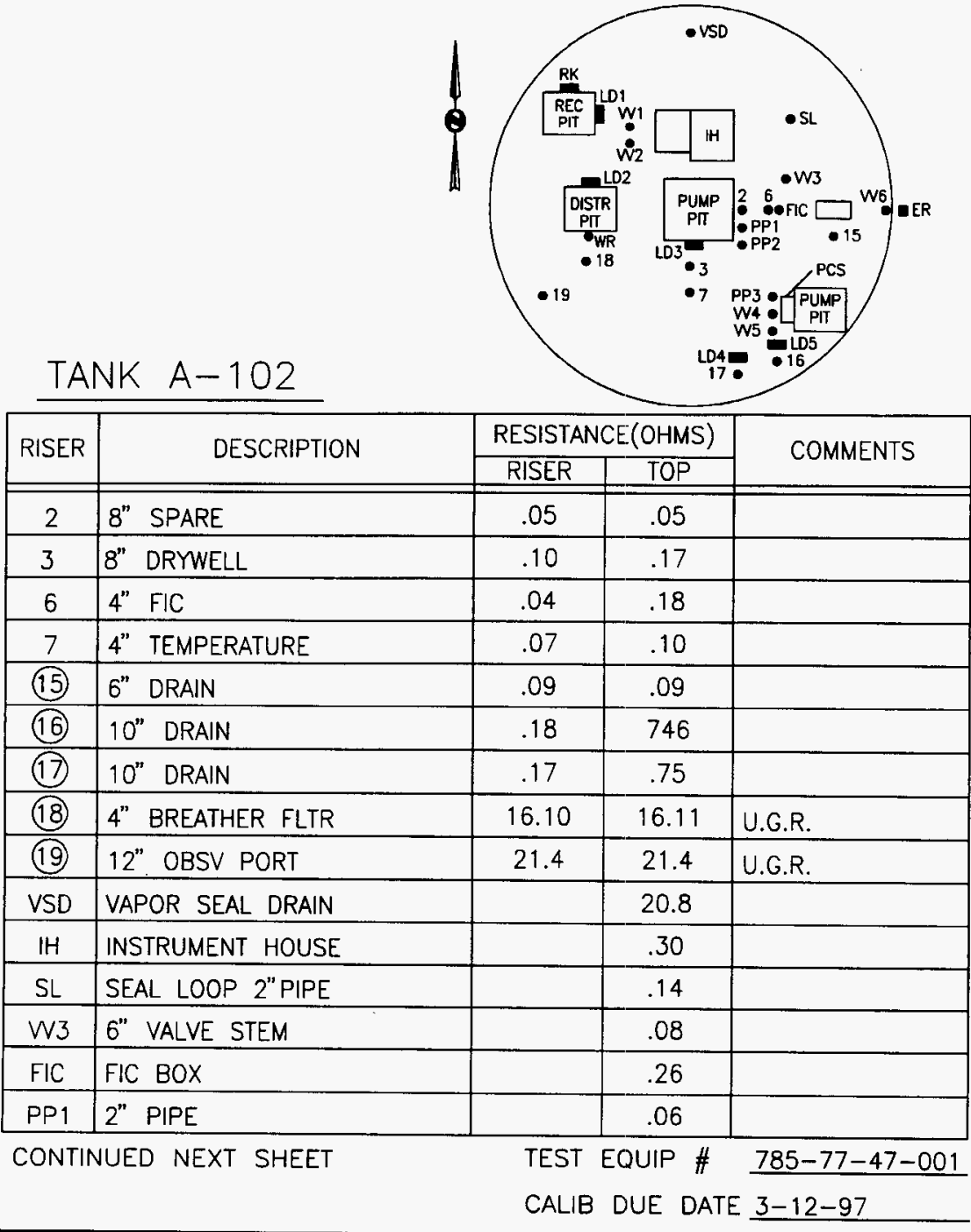




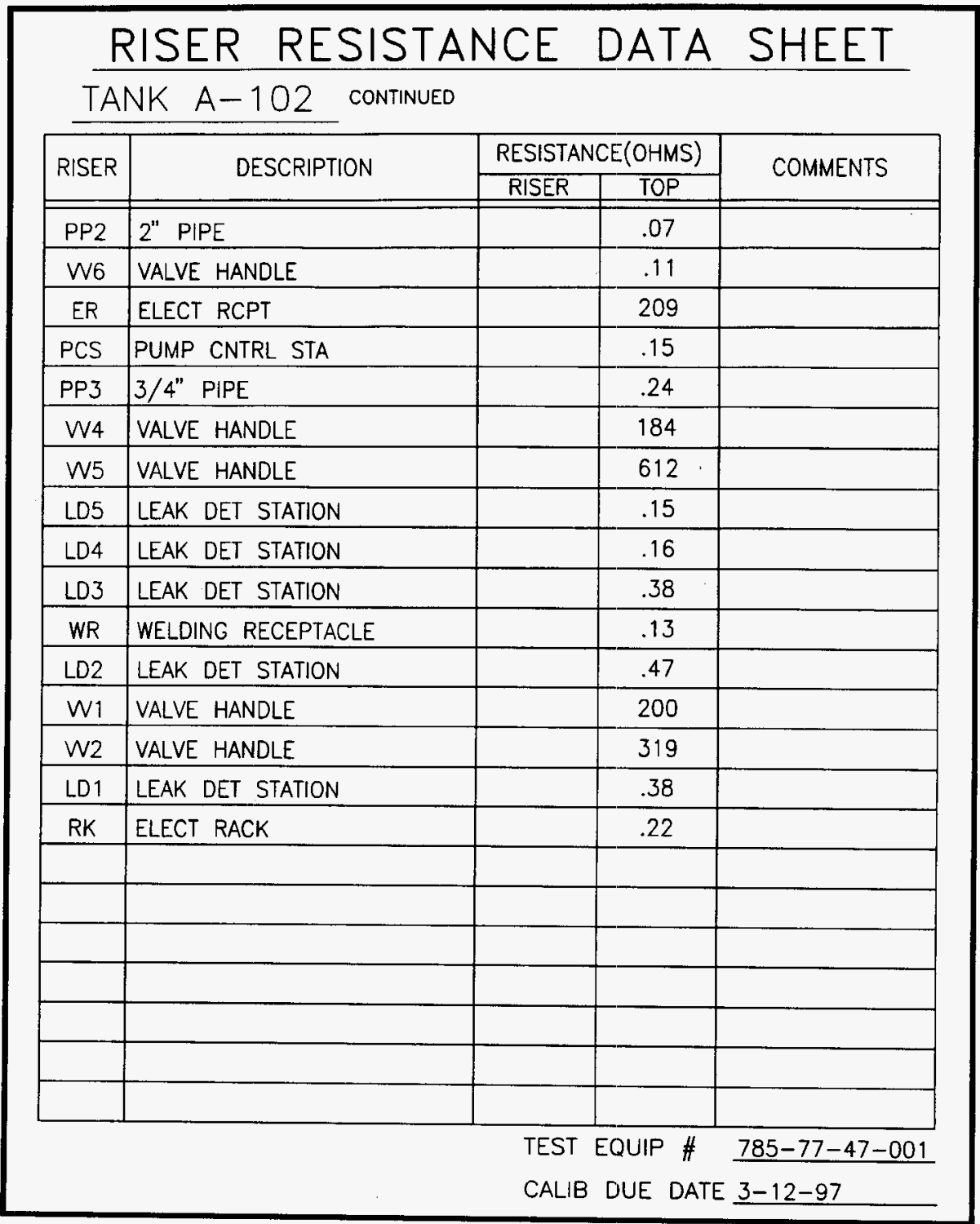




\section{RISER RESISTANCE DATA SHEET}

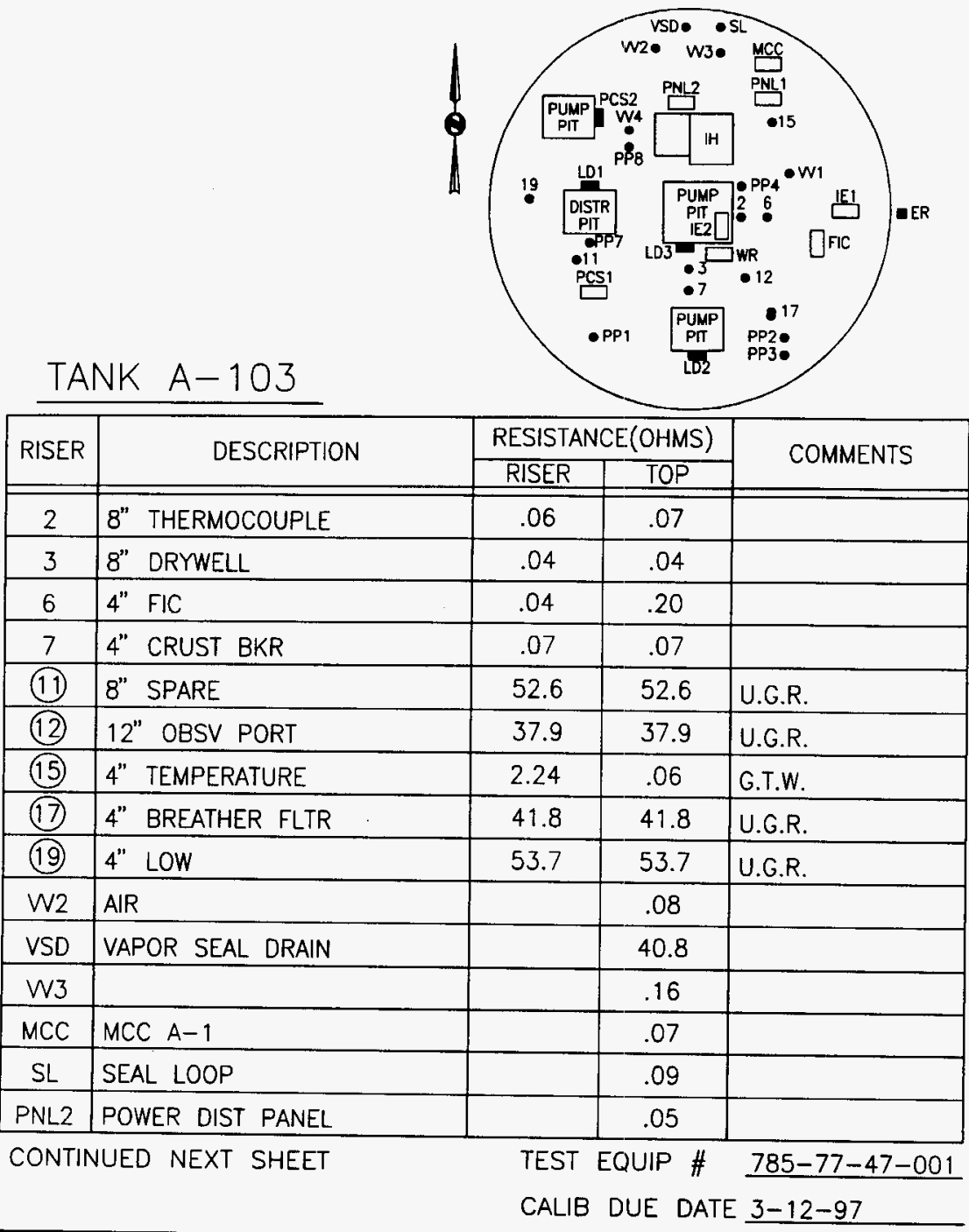




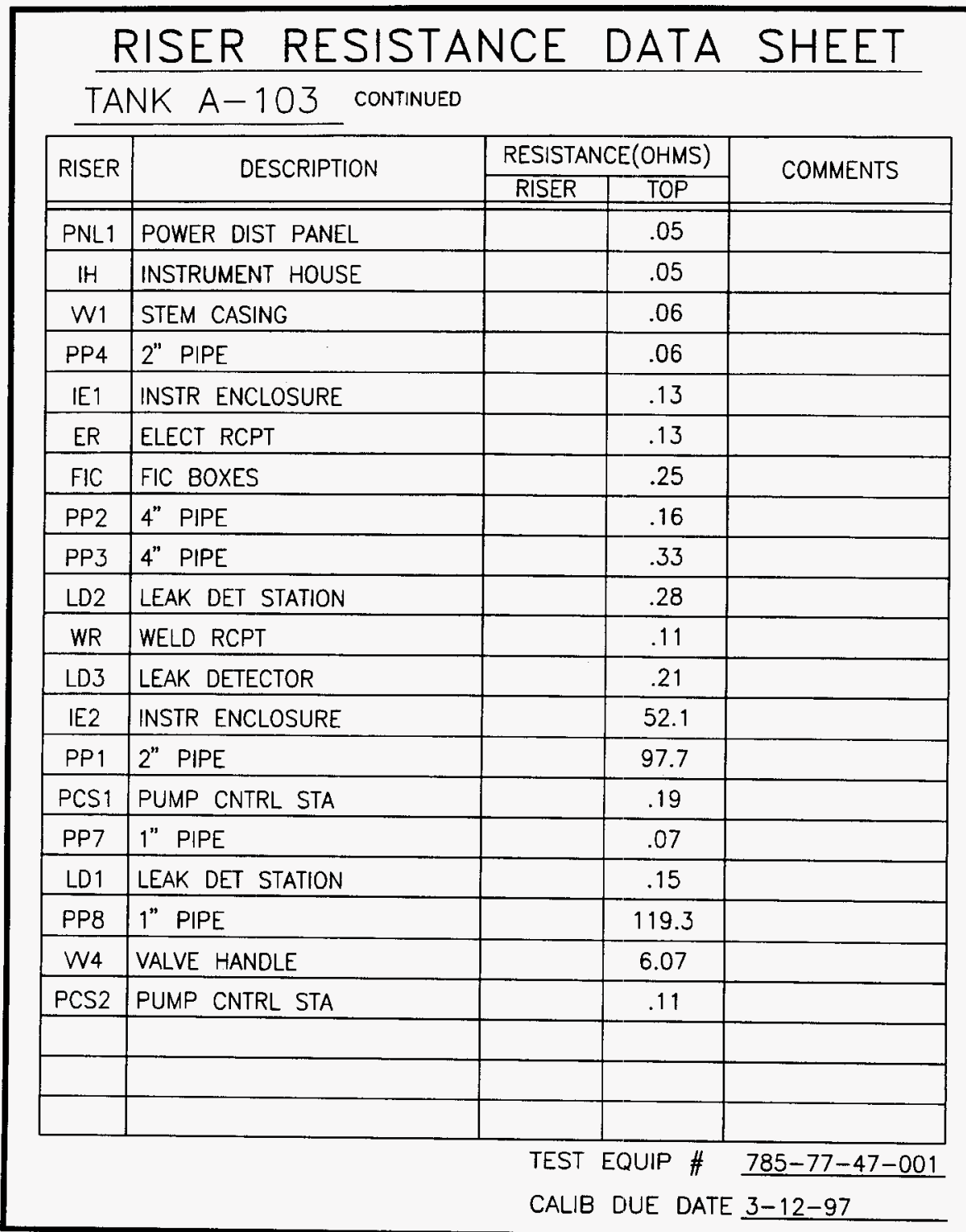




\section{RISER RESISTANCE DATA SHEET}

TANK A-104
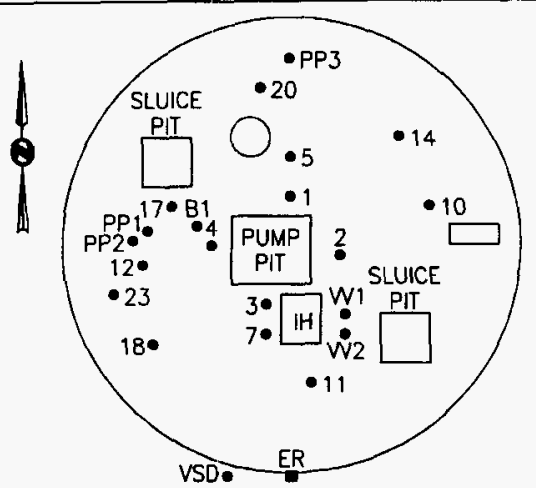

\begin{tabular}{|c|c|c|c|c|}
\hline \multirow{2}{*}{ RISER } & \multirow{2}{*}{ DESCRIPTION } & \multicolumn{2}{|c|}{ RESISTANCE(OHMS) } & \multirow{2}{*}{ COMMENTS } \\
\hline & & RISER & TOP & \\
\hline 1 & 8" SPARE & .06 & .06 & \\
\hline 2 & 8" THERMOCOUPLE & .06 & 8.59 & \\
\hline 3 & 12" AIR & .07 & .07 & \\
\hline 4 & $12^{n}$ OBSV PORT & .06 & .06 & \\
\hline 5 & $4^{\prime \prime}$ CRUST BRKR & .15 & .18 & \\
\hline 7 & 4" SPARE & .07 & .07 & \\
\hline (10) & $2.5^{n}$ SLUDGE MEAS & 24.7 & 24.8 & U.G.R. \\
\hline (11) & $2.5^{\prime \prime}$ SPARE & 25.7 & OPEN & U.G.R. \\
\hline (12) & 2.5" TEMP ELEMENT & 23.5 & 23.5 & U.G.R. \\
\hline (14) & $4^{\prime \prime} \quad$ BREATHER FLTR & 19.2 & 19.3 & U.G.R. \\
\hline (17) & 12" SPARE & 87.3 & 87.3 & U.G.R. \\
\hline (18) & 4" TEMP ELEMENT & 21.6 & 21.6 & U.G.R. \\
\hline (20) & $2.5^{\prime \prime}$ SLUDGE MEAS & 23.5 & 23.9 & U.G.R. \\
\hline (23) & $4 "$ SPARE & 23.4 & 23.5 & U.G.R. \\
\hline PP3 & 2" PIPE & & .46 & \\
\hline
\end{tabular}

CONTINUED NEXT SHEET

TEST EQUIP \# 785-77-47-001 CALIB DUE DATE $3-12-97$ 


\section{RISER RESISTANCE DATA SHEET}

TANK A-104 CONTINUED

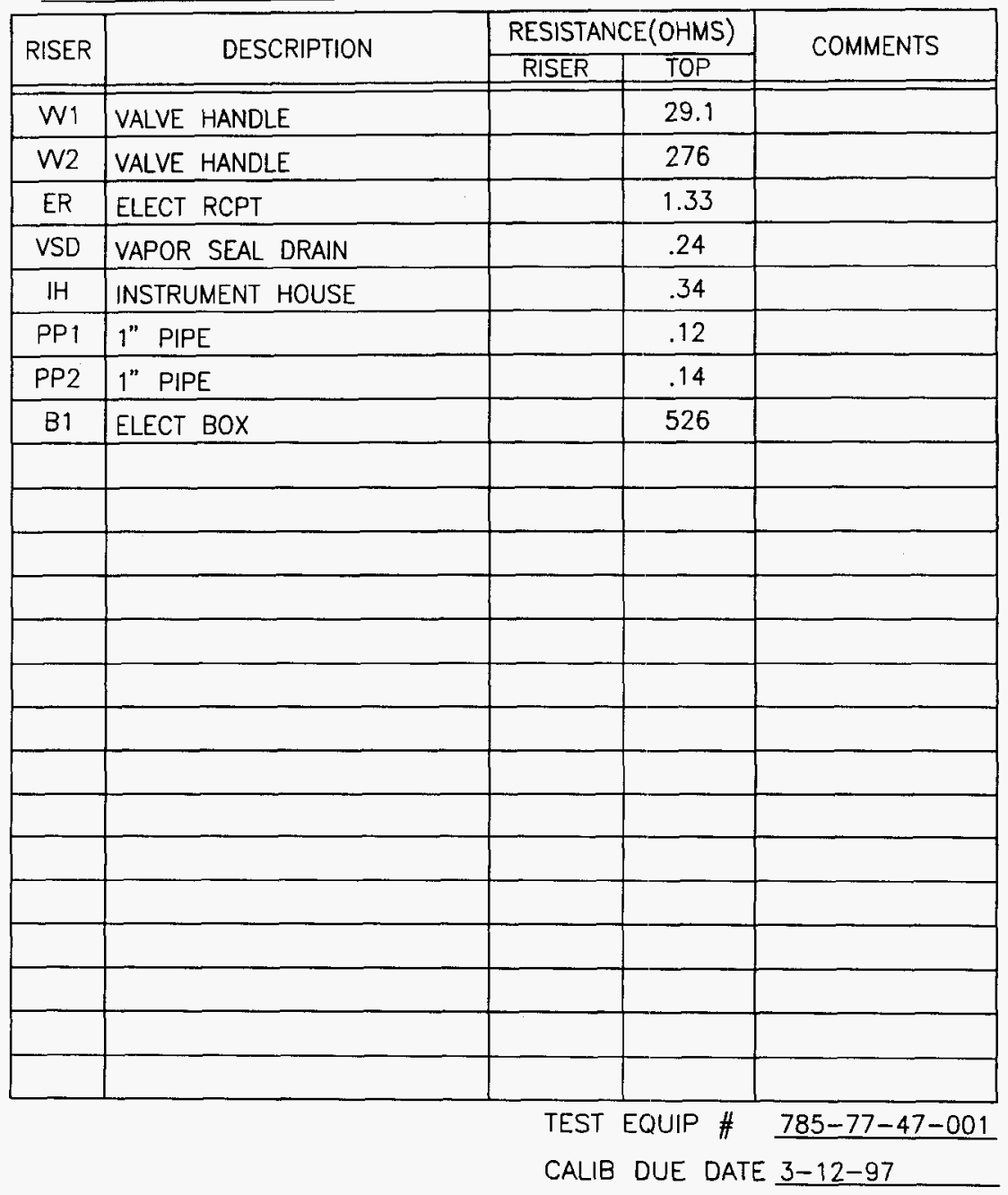




\section{RISER RESISTANCE DATA SHEET}

TANK $A-105$

\begin{tabular}{|c|c|c|c|c|}
\hline \multirow{2}{*}{ RISER } & \multirow{2}{*}{ DESCRIPTION } & \multicolumn{2}{|c|}{ RESISTANCE(OHMS) } & \multirow{2}{*}{ COMMENTS } \\
\hline & & RISER & TOP & \\
\hline 1 & 8" SPARE & .05 & .24 & \\
\hline 5 & 4" REEL & .05 & OPEN & \\
\hline 8 & 4" PRESS GAUGE & .04 & .05 & \\
\hline (9) & 8" TEMPERATURE & 22.2 & 22.2 & U.G.R. \\
\hline (14) & 4" SPARE & 16.24 & 16.24 & U.G.R. \\
\hline (15) & 4" TEMPERATURE & 189 & 189 & U.G.R. \\
\hline 16 & 4" TEMPERATURE & 76.8 & 76.8 & U.G.R. \\
\hline (17) & 4" TEMPERATURE & 254 & 254 & U.G.R. \\
\hline (18) & 4" SPARE & 15.09 & 15.09 & U.G.R. \\
\hline (19) & 4" TEMPERATURE & $3.76 \mathrm{~K}$ & $3.76 \mathrm{~K}$ & U.G.R. \\
\hline (20) & 4" SPARE & 62.6 & 62.6 & U.G.R. \\
\hline 22 & 4" TEMPERATURE & OPEN & & U.G.R. \\
\hline ES & EXHAUST STACK & & .09 & \\
\hline $\mathrm{IH}$ & INSTRUMENT HOUSE & & .17 & \\
\hline P1 & 2" PIPE & 2.10 & 2.10 & \\
\hline
\end{tabular}

TEST EQUIP \# 785-77-47-001

CALIB DUE DATE 3-12-97 


\section{RISER RESISTANCE DATA SHEET}

TANK $A-106$

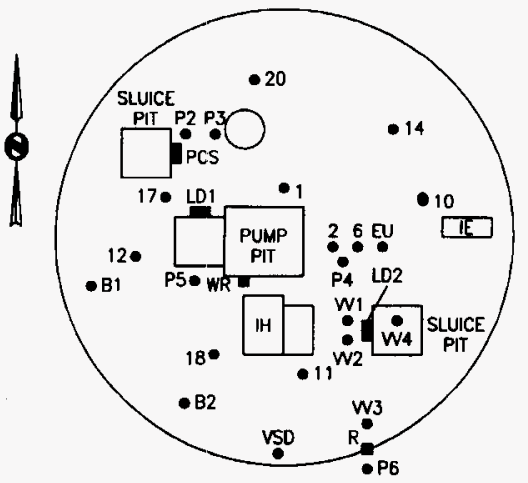

\begin{tabular}{|c|c|c|c|c|}
\hline \multirow{2}{*}{ RISER } & \multirow{2}{*}{ DESCRIPTION } & \multicolumn{2}{|c|}{ RESISTANCE(OHMS) } & \multirow{2}{*}{ COMMENTS } \\
\hline & & RISER & TOP & \\
\hline 1 & 8" DRYWELL & .02 & .03 & \\
\hline 2 & 8" THERMOCOUPLE & .13 & 1.18 & \\
\hline 6 & 4" ENRAF & .11 & .41 & \\
\hline (10) & 6" SLUDGE MEAS & 18.0 & 21.5 & U.G.R. \\
\hline (11) & 6" SLUDGE MEAS & 27.6 & 28.9 & U.G.R. \\
\hline (12) & 6" SPARE & 33.4 & 33.5 & U.G.R. \\
\hline (14) & 4" TEMPERATURE & 3.34 & .12 & G.T.W. \\
\hline (17) & 12" OBSV PORT & 22.1 & 22.5 & U.G.R. \\
\hline (18) & 4" BREATHER FLTR & 33.4 & 33.5 & U.G.R. \\
\hline 20 & 6" SPARE & .08 & 2.37 & \\
\hline IE & INSTR ENCLOSURE & & .05 & \\
\hline EU & ENRAF UNISTRUT & & .37 & \\
\hline $\mathrm{P} 4$ & 2" PIPE & & 472 & \\
\hline$W 1$ & VALVE HANDLE & & 12 & \\
\hline W2 & VALVE HANDLE & & .06 & \\
\hline
\end{tabular}

CONTINUED NEXT SHEET

TEST EQUIP \# 785-77-47-001 CALIB DUE DATE 3-12-97 


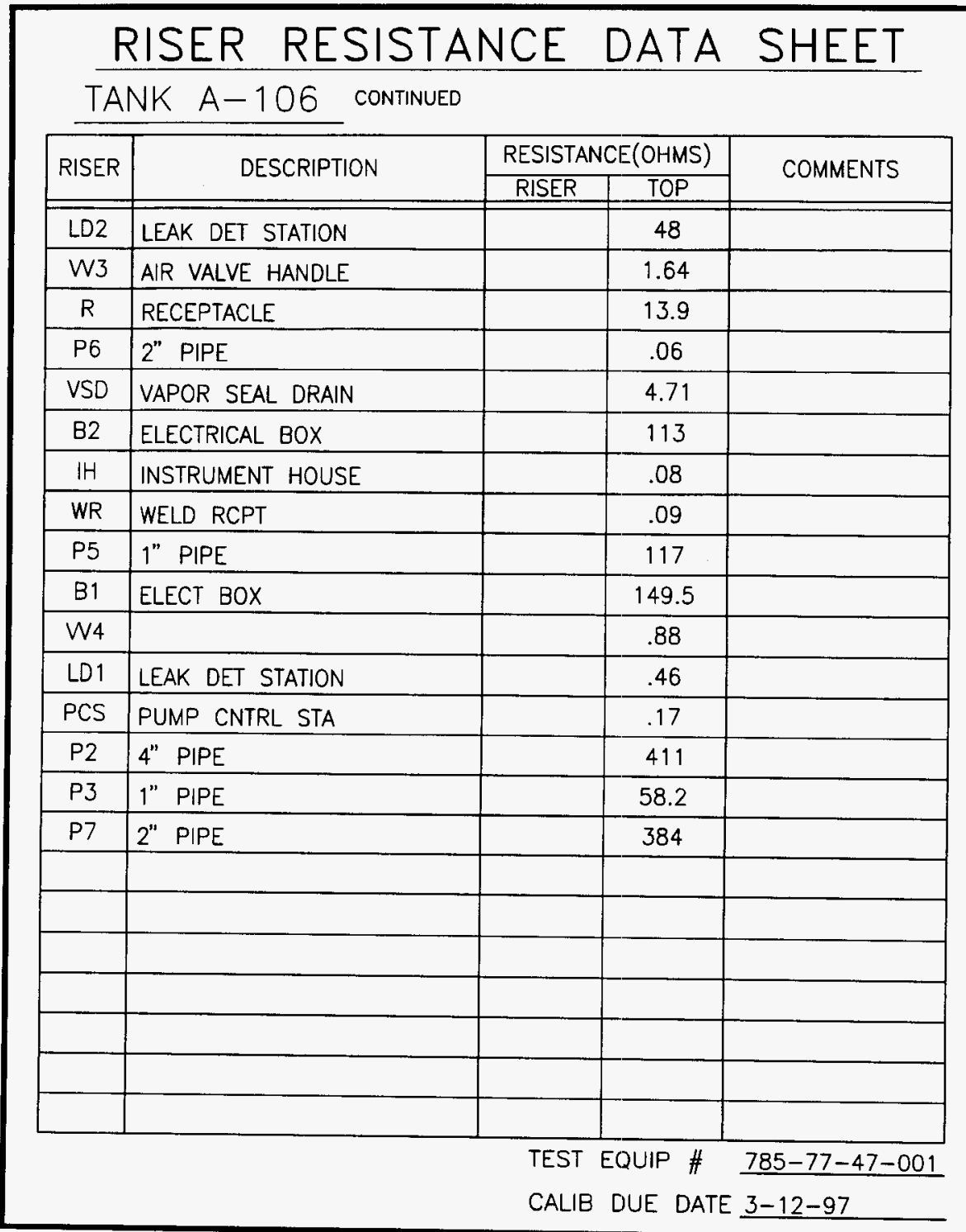


WHC-SD-WM-TR-034, REV. 0

\section{RISER RESISTANCE DATA SHEET}

TANK AX-101
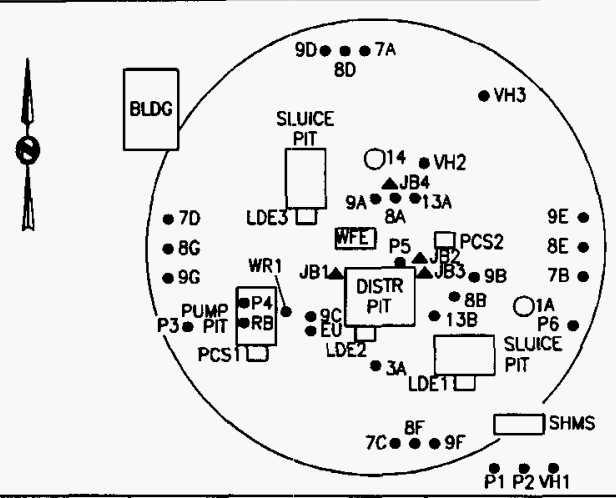

\begin{tabular}{|c|c|c|c|c|}
\hline \multirow{2}{*}{ RISER } & \multirow{2}{*}{ DESCRIPTION } & \multicolumn{2}{|c|}{ RESISTANCE(OHMS) } & \multirow{2}{*}{ COMMENTS } \\
\hline & & RISER & TOP & \\
\hline $1 \mathrm{~A}$ & 34" STEAM COIL CAISSON & & 19.31 & \\
\hline $3 \mathrm{~A}$ & 16" OBSV PORT & & & TOO HOT \\
\hline $7 \mathrm{~A}$ & 4" PROFILE TEMP & & & RISER ENCASED \\
\hline $7 \mathrm{~B}$ & 4" PROFILE TEMP & & & RISER ENCASED \\
\hline $7 C$ & 4" PROFILE TEMP & & & RISER ENCASED \\
\hline 70 & 4" PROFILE TEMP & & & RISER ENCASED \\
\hline $8 \mathrm{~A}$ & $6 "$ PLUG & .46 & 2.34 & \\
\hline $8 \mathrm{~B}$ & 6" PLUG & .45 & 48.5 & \\
\hline 80 & $6^{\prime \prime}$ PLUG & .48 & .80 & \\
\hline $8 \mathrm{E}$ & 6" PLUG & .45 & 2.17 & \\
\hline $8 \mathrm{~F}$ & $6^{\prime \prime}$ SPARE & .47 & 5.51 & \\
\hline 86 & 6" PLUG & .47 & OPEN & \\
\hline $9 A$ & 6" LOW & .47 & .53 & \\
\hline $9 B$ & $6^{\prime \prime}$ TEMP PROBE & .45 & .48 & \\
\hline $9 \mathrm{C}$ & 6" ENRAF & .50 & .41 & \\
\hline \multicolumn{2}{|c|}{ CONTINUED NEXT SHEET } & \multicolumn{3}{|c|}{$\begin{array}{l}\text { TEST EQUIP \# } \frac{785-77-47-001}{3-12-97} \\
\text { CALIB DUE DATE }\end{array}$} \\
\hline
\end{tabular}




\section{RISER RESISTANCE DATA SHEET}

TANK AX-101 CONTINUED

\begin{tabular}{|c|c|c|c|c|}
\hline \multirow{2}{*}{ RISER } & \multirow{2}{*}{ DESCRIPTION } & \multicolumn{2}{|c|}{ RESISTANCE(OHMS) } & \multirow{2}{*}{ COMMENTS } \\
\hline & & RISER & TOP & \\
\hline 90 & 6" SLUDGE MEAS & .47 & $17.5 \mathrm{~K}$ & \\
\hline $9 E$ & 6" BREATHER FLTR & .46 & .48 & \\
\hline $9 F$ & 6" SHMS CONNECT & .47 & .47 & \\
\hline $9 \mathrm{G}$ & 6" SLUDGE MEAS & .45 & .46 & \\
\hline $13 \mathrm{~A}$ & 4" WC & & & RISER ENCASED \\
\hline $13 B$ & 4" WC & & & RISER ENCASED \\
\hline 14 & 42" STEAM COIL CAISSON & & 14.83 & \\
\hline $\mathrm{VH} 3$ & VALVE & $* 140.6$ & $8.98 \mathrm{~K}$ & *CASING \\
\hline $\mathrm{VH} 2$ & VALVE HANDLE & & .46 & \\
\hline $\mathrm{JB} 4$ & $\mathrm{JBOX}(20 \times 20)$ & & 272 & \\
\hline WFE & WF ENCLOSURE & & .43 & \\
\hline P5 & AIR VALVE & & 320 & \\
\hline $\mathrm{JB} 2$ & $\mathrm{~J} B O x$ & & OPEN & \\
\hline J83 & $\mathrm{J}$ BOX & & .49 & \\
\hline PCS2 & PUMP CNTRL STA & & .68 & \\
\hline P6 & 2" PIPE W/FLANGE & .46 & .47 & \\
\hline LDE2 & LEAK DETECTION & & .58 & \\
\hline LDE 1 & LEAK DETECTION & & .61 & \\
\hline SHMS & SHMS CABINET & & .50 & \\
\hline $\mathrm{VH} 1$ & VALVE HANDLE & & 963 & \\
\hline $\mathrm{P} 2$ & 2" PIPE & & 219 & \\
\hline $\mathrm{P} 1$ & $2^{n}$ PIPE & & 218 & \\
\hline PCS1 & PUMP CNTRL STA & & .50 & \\
\hline \multicolumn{2}{|c|}{ CONTINUED NEXT SHEET } & \multicolumn{2}{|c|}{ TEST EQUIP \# } & $\begin{array}{l}785-77-47-001 \\
3-12-97\end{array}$ \\
\hline
\end{tabular}




\section{RISER RESISTANCE DATA SHEET}

TANK AX-101 CONTINUED

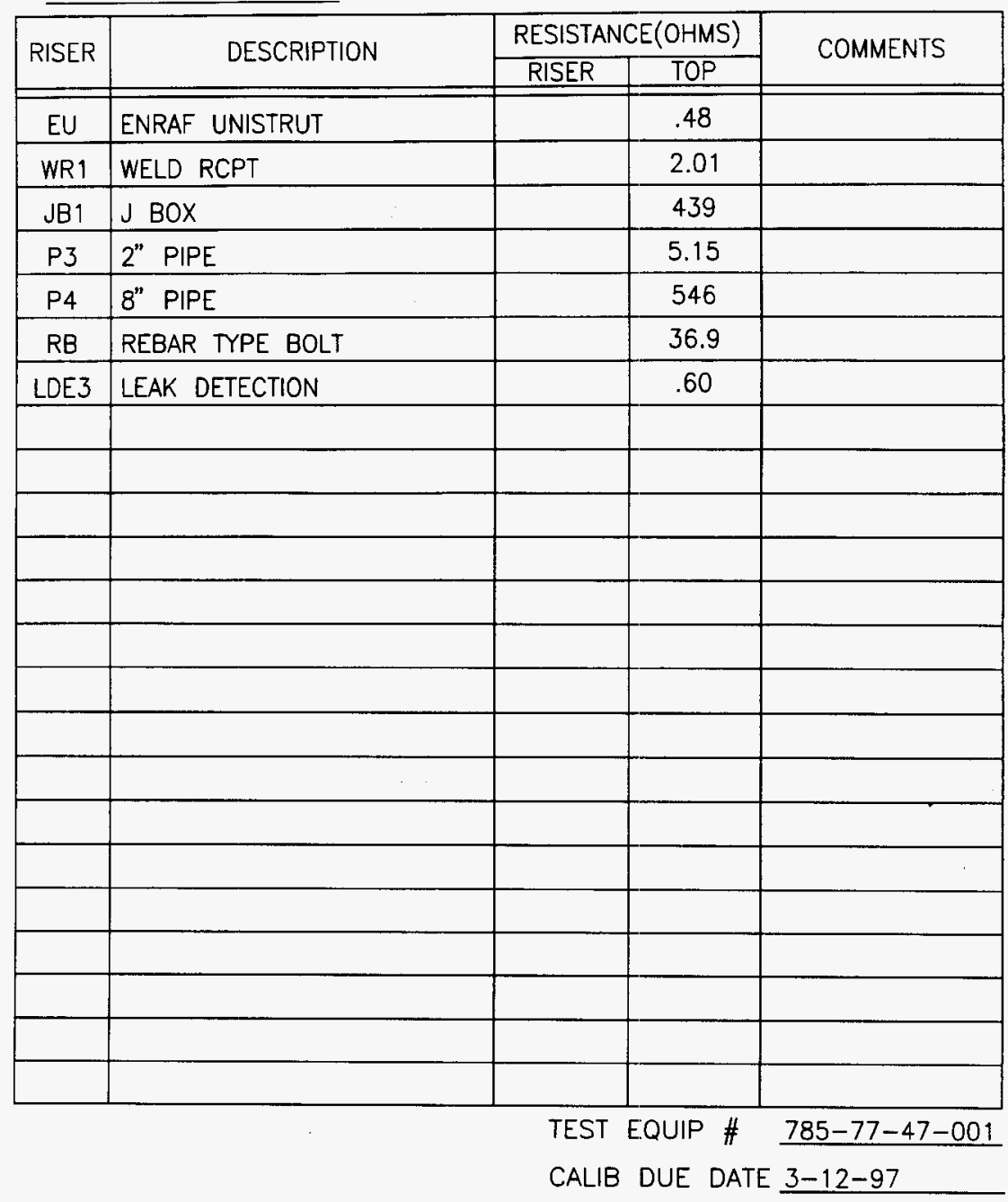




\section{RISER RESISTANCE DATA SHEET}

\begin{tabular}{|c|c|c|c|c|}
\hline \multirow{2}{*}{ RISER } & \multirow{2}{*}{ DESCRIPTION } & \multicolumn{2}{|c|}{ RESISTANCE(OHMS) } & \multirow{2}{*}{ COMMENTS } \\
\hline & & RISER & TOP & \\
\hline $3 A$ & 17" OBSV PORT & .48 & OPEN & \\
\hline $7 \mathrm{~A}$ & 4" TEMPERATURE & .47 & .60 & \\
\hline $7 \mathrm{~B}$ & 4" TEMPERATURE & .47 & .62 & \\
\hline $7 \mathrm{C}$ & 4" TEMPERATURE & .46 & .51 & \\
\hline 70 & 4" TEMPERATURE & .46 & .48 & \\
\hline $8 \mathrm{~A}$ & 6" PLUG & .46 & .46 & \\
\hline $8 \mathrm{~B}$ & 6" PLUG & .47 & $4.85 \mathrm{~K}$ & \\
\hline $8 \mathrm{C}$ & 6" PLUG & .46 & 8.44 & \\
\hline 80 & 6" PLUG & .46 & .68 & \\
\hline $8 E$ & 6" PLUG & .46 & 6.24 & \\
\hline $8 \mathrm{~F}$ & 6" PLUG & .46 & 469 & \\
\hline $8 G$ & 6" PLUG & .46 & 19.74 & \\
\hline $9 A$ & 6" SLUDGE MEAS & .46 & .46 & \\
\hline $9 \mathrm{~B}$ & 6" $\mathrm{FIC}$ & .46 & .50 & \\
\hline $9 \mathrm{C}$ & 6" TEMPERATURE & & & RISER ENCASED \\
\hline \multicolumn{2}{|c|}{ CONTINUED NEXT SHEET } & \multicolumn{3}{|c|}{$\begin{array}{l}\text { TEST EQUIP \# } 785-77-47-001 \\
\text { CALIB DUE DATE } 3-12-97\end{array}$} \\
\hline
\end{tabular}




\section{RISER RESISTANCE DATA SHEET}

TANK AX-102 CONTINUED

\begin{tabular}{|c|c|c|c|c|}
\hline \multirow{2}{*}{ RISER } & \multirow{2}{*}{ DESCRIPTION } & \multicolumn{2}{|c|}{ RESISTANCE(OHMS) } & \multirow{2}{*}{ COMMENTS } \\
\hline & & RISER & TOP & \\
\hline 90 & 6" LLR & .46 & 4.04 & \\
\hline $9 E$ & 6" BREATHER FLTR & .46 & .48 & \\
\hline $9 \mathrm{~F}$ & 6" SPARE & .47 & .51 & \\
\hline $9 G$ & 6" SLUDGE MEAS & .47 & 640 & \\
\hline $13 \mathrm{~A}$ & 4" WC & & & RISER ENCASED \\
\hline $13 \mathrm{~B}$ & 4" TEMPERATURE & .46 & .53 & \\
\hline $13 \mathrm{C}$ & 4" WC & .46 & 1.43 & \\
\hline 14 & 42" STEAM COIL CAISSON & & 16.11 & \\
\hline P2 & 1" PIPE & & $1.746 \mathrm{~K}$ & \\
\hline $\mathrm{FIC}$ & FIC BOX & & & \\
\hline LDE3 & LEAK DETECTION & & .79 & \\
\hline WR2 & WELD RCPT & & 6.68 & \\
\hline $\mathrm{P} 1$ & 2" PIPE & & .56 & \\
\hline LDE2 & LEAK DETECTION & & .67 & \\
\hline JB1 & $J B 0 \times(20 \times 20)$ & & 232 & \\
\hline BLDG & BUILDING & & .50 & \\
\hline WR1 & WELD RCPT & & 33.6 & \\
\hline PCS & PUMP CNTRL STA & & .57 & \\
\hline LDE 1 & LEAK DETECTION & & .62 & \\
\hline WR3 & WELD RCPT & & 3.35 & \\
\hline WR4 & $60 \mathrm{~A}$ RCPT & & 1.66 & \\
\hline $\mathrm{VH} 1$ & VALVE HANDLE & $* 52.52$ & 157.3 & *CASING \\
\hline & & $\begin{array}{l}\text { TEST } \\
\text { CALIB }\end{array}$ & $\begin{array}{l}\text { QUIP \# } \\
\text { DUE DAT }\end{array}$ & $\begin{aligned} & 785-77-47-001 \\
= & 3-12-97\end{aligned}$ \\
\hline
\end{tabular}




\section{RISER RESISTANCE DATA SHEET}

TANK AX-103

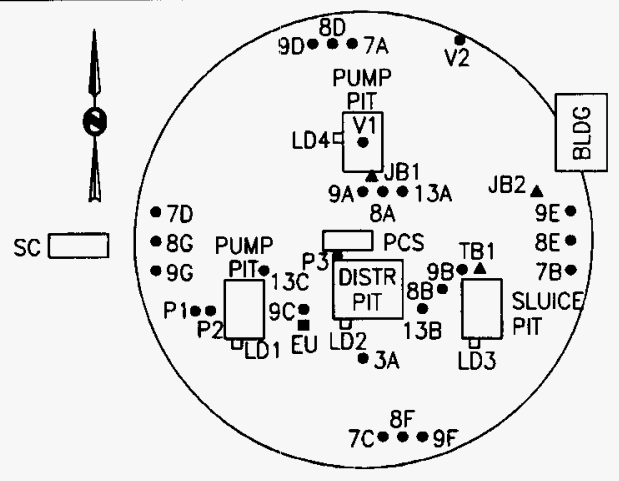

\begin{tabular}{|c|c|c|c|c|}
\hline \multirow{2}{*}{ RISER } & \multirow{2}{*}{ DESCRIPTION } & \multicolumn{2}{|c|}{ RESISTANCE(OHMS) } & \multirow{2}{*}{ COMMENTS } \\
\hline & & RISER & TOP & \\
\hline $3 A$ & $16^{\prime \prime}$ OBSV PORT & & & NO ACCESS \\
\hline $7 \mathrm{~A}$ & 4" TEMPERATURE & .50 & .55 & \\
\hline $7 \mathrm{~B}$ & 4" TEMPERATURE & .45 & .49 & \\
\hline $7 C$ & 4" TEMPERATURE & .45 & .62 & \\
\hline $7 D$ & 4" TEMPERATURE & .48 & .60 & \\
\hline $8 \mathrm{~A}$ & 6" DRYWELL & .46 & 11.7 & \\
\hline $8 \mathrm{~B}$ & $6^{\prime \prime}$ PLUG & .45 & .55 & \\
\hline 80 & 6" SPARE & .46 & .50 & \\
\hline $8 E$ & 6" SPARE & .45 & .54 & \\
\hline $8 \mathrm{~F}$ & 6" SPARE & .45 & 1.89 & \\
\hline 86 & 6" SPARE & .47 & 1.13 & \\
\hline $9 A$ & 6" SPARE & .47 & $5.6 \mathrm{~K}$ & \\
\hline $9 \mathrm{~B}$ & 6" TEMPERATURE & .46 & .58 & \\
\hline $9 \mathrm{C}$ & 6" ENRAF & .50 & .50 & \\
\hline 90 & 6" SLUDGE MEAS & .45 & 118.3 & \\
\hline
\end{tabular}

CONTINUED ON SHEET 2

TEST EQUIP \# 785-77-47-001

CALIB DUE DATE 3-12-97 


\section{RISER RESISTANCE DATA SHEET}

TANK AX-103 CONTINUED FROM SH 1

\begin{tabular}{|c|c|c|c|c|}
\hline \multirow{2}{*}{ RISER } & \multirow{2}{*}{ DESCRIPTION } & \multicolumn{2}{|c|}{ RESISTANCE(OHMS) } & \multirow{2}{*}{ COMMENTS } \\
\hline & & RISER & TOP & \\
\hline $9 \mathrm{E}$ & 6" BREATHER FLTR & .46 & .47 & \\
\hline $9 \mathrm{~F}$ & 6" SPARE & .45 & .47 & \\
\hline $9 G$ & 6" SPARE & .46 & .49 & \\
\hline $13 \mathrm{~A}$ & 4" TEMPERATURE & .46 & .53 & \\
\hline 138 & 4" WC & & & RISER ENCASED \\
\hline $13 \mathrm{C}$ & 4" TEMPERATURE & & & RISER ENCASED \\
\hline $\mathrm{V} 2$ & VALVE HANDLE & $* .65$ & 1.14 & *CASING \\
\hline LD4 & LEAK DETECTION & & .58 & \\
\hline$V_{1}$ & VALVE HANDLE & & 3.55 & \\
\hline JB1 & $24^{\prime \prime} \times 30^{\prime \prime} \mathrm{J}-\mathrm{BOX}$ & & .16 & \\
\hline JB2 & JUNCTION BOX & & 8.97 & \\
\hline BLDG & BUILDING & & .45 & \\
\hline $\mathrm{SC}$ & SHMS & & .56 & \\
\hline PCS & PUMP CONTROL STA & & .79 & \\
\hline P3 & 1" PIPE/VALVE & & 63.4 & \\
\hline TB1 & TEMP BOX & & 413 & \\
\hline $\mathrm{P} 1$ & 8" PIPE & & 50.9 & \\
\hline $\mathrm{P} 2$ & 8" PIPE & & 165.2 & \\
\hline EU & ENRAF UNISTRUT & & .50 & \\
\hline LD2 & LEAK DETECTION & & .46 & \\
\hline LD3 & LEAK DETECTION & & .54 & \\
\hline LD1 & LEAK DETECTION & & .50 & \\
\hline
\end{tabular}

TEST EQUIP \# 785-77-47-001

CALIB DUE DATE 3-12-97 


\section{RISER RESISTANCE DATA SHEET}

\begin{tabular}{|c|c|c|c|c|}
\hline \multirow{2}{*}{ RISER } & \multirow{2}{*}{ DESCRIPTION } & \multicolumn{2}{|c|}{ RESISTANCE(OHMS) } & \multirow{2}{*}{ COMMENTS } \\
\hline & & RISER & TOP & \\
\hline $3 \mathrm{~A}$ & 14" OBSV PORT & .47 & .47 & \\
\hline $7 \mathrm{~A}$ & 4" TEMPERATURE & .44 & .56 & \\
\hline $7 \mathrm{~B}$ & 4" TEMPERATURE & .46 & .47 & \\
\hline $8 \mathrm{~A}$ & 6" DRYWELL & .45 & .52 & \\
\hline $8 \mathrm{~B}$ & 6" SPARE & .46 & .52 & \\
\hline $8 \mathrm{C}$ & 6" DRYWELL & .47 & .56 & \\
\hline 80 & 6" DRYWELLL & .45 & 484 & \\
\hline $8 \mathrm{E}$ & 6" DRYWELL & .46 & .46 & \\
\hline $8 F$ & 6" PLUG & .45 & 1.82 & \\
\hline $8 G$ & 6" PLUG & & 5.14 & BOTTOM NO ACCESS \\
\hline $9 \mathrm{~A}$ & 6" REEL & .45 & .45 & \\
\hline $9 \mathrm{~B}$ & 6" FIC & .47 & .52 & \\
\hline $9 \mathrm{C}$ & 6" TEMPERATURE & .45 & 1.67 & \\
\hline $9 E$ & $6^{\prime \prime}$ BREATHER FLTR & .50 & .50 & \\
\hline $9 \mathrm{~F}$ & $6^{\prime \prime}$ & .46 & 27.6 & \\
\hline \multicolumn{2}{|c|}{ CONTINUED ON SHEET 2} & \multicolumn{3}{|c|}{$\begin{array}{l}\text { TEST EQUIP \# } \frac{785-77-47-001}{3-12-97} \\
\text { CALIB DUE DATE }\end{array}$} \\
\hline
\end{tabular}




\section{RISER RESISTANCE DATA SHEET}

TANK $A X-104$ CONTINUED FROM SHEET 1

\begin{tabular}{|c|c|c|c|c|}
\hline \multirow{2}{*}{ RISER } & \multirow{2}{*}{ DESCRIPTION } & \multicolumn{2}{|c|}{ RESISTANCE(OHMS) } & \multirow{2}{*}{ COMMENTS } \\
\hline & & RISER & TOP & \\
\hline $9 G$ & $6^{\prime \prime}$ SLUDGE MEAS & & 1.64 & BOTTOM NO ACCESS \\
\hline $13 \mathrm{~A}$ & 4" WC & & & RISER ENCASED \\
\hline $13 \mathrm{~B}$ & 4" WC & & & RISER ENCASED \\
\hline $13 \mathrm{C}$ & 4" WC & & & RISER ENCASED \\
\hline (16A) & $4^{\prime \prime} \quad B G, 2 " W / F L G$ & 42.6 & 44.7 & U.G.R. \\
\hline (16B) & 4" SPARE, 2" W/FLG & 44.2 & 44.2 & U.G.R. \\
\hline$(160)$ & 4" SPARE, 2" W/FLG & 4.70 & 4.70 & \\
\hline P7 & $3^{\prime \prime}$ PIPE & & 41.4 & \\
\hline P5 & 2" PIPE & & .53 & \\
\hline $\mathrm{Pg}$ & 2" PIPE & & $1.7 \mathrm{~K}$ & \\
\hline P8 & 2" PIPE & & $1.41 \mathrm{~K}$ & \\
\hline LD1 & LEAK DETECTION & & .52 & \\
\hline LD3 & LEAK DETECTION & & .58 & \\
\hline$V_{4}$ & VALVE HANDLE & & 12.4 & \\
\hline LD2 & LEAK DETECTION & & .57 & \\
\hline $\mathrm{P} 4$ & 1" PIPE & & 218 & \\
\hline V3 & VALVE HANDLE & & 12.7 & \\
\hline $\mathrm{V} 2$ & VALVE HANDLE & & 12.6 & \\
\hline V5 & 1" PIPE W/VALVE & & 368 & \\
\hline WR1 & WELDING RECEPTACLE & & 4.85 & \\
\hline P6 & 2" PIPE & & .52 & \\
\hline $\mathrm{FIC}$ & FIC BOX & & .48 & \\
\hline PCS & PUMP CONTROL STA & & .58 & \\
\hline & CONTINUED ON SHEET 3 & $\begin{array}{l}\text { TEST } \\
\text { CALI }\end{array}$ & $\begin{array}{l}\text { QUIP \# } \\
\text { OUE DAT }\end{array}$ & $\begin{array}{l}785-77-47-001 \\
3-12-97\end{array}$ \\
\hline
\end{tabular}




\section{RISER RESISTANCE DATA SHEET}

TANK AX-104 CONTINUED FROM SHEET 2

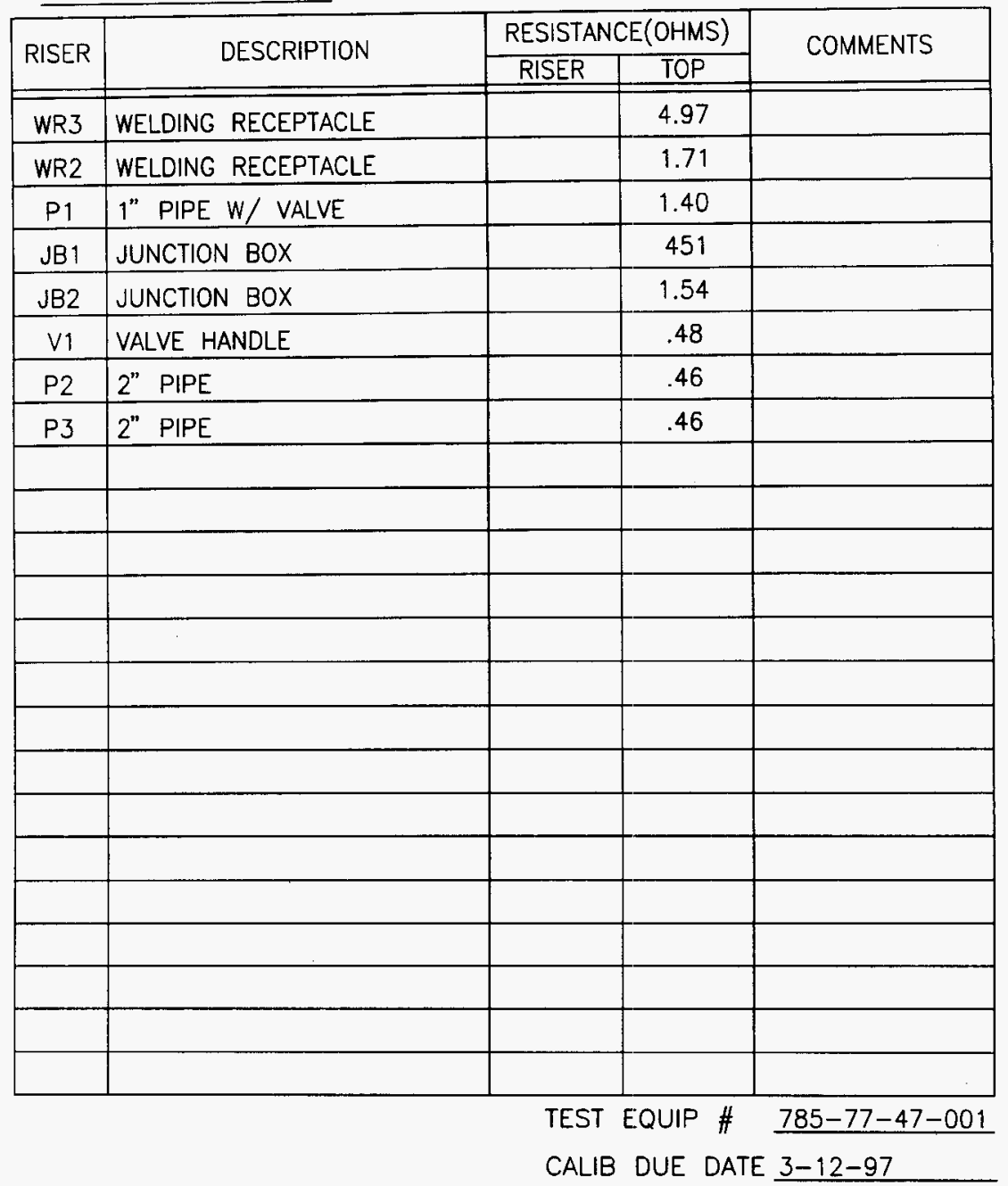




\section{RISER RESISTANCE DATA SHEET}

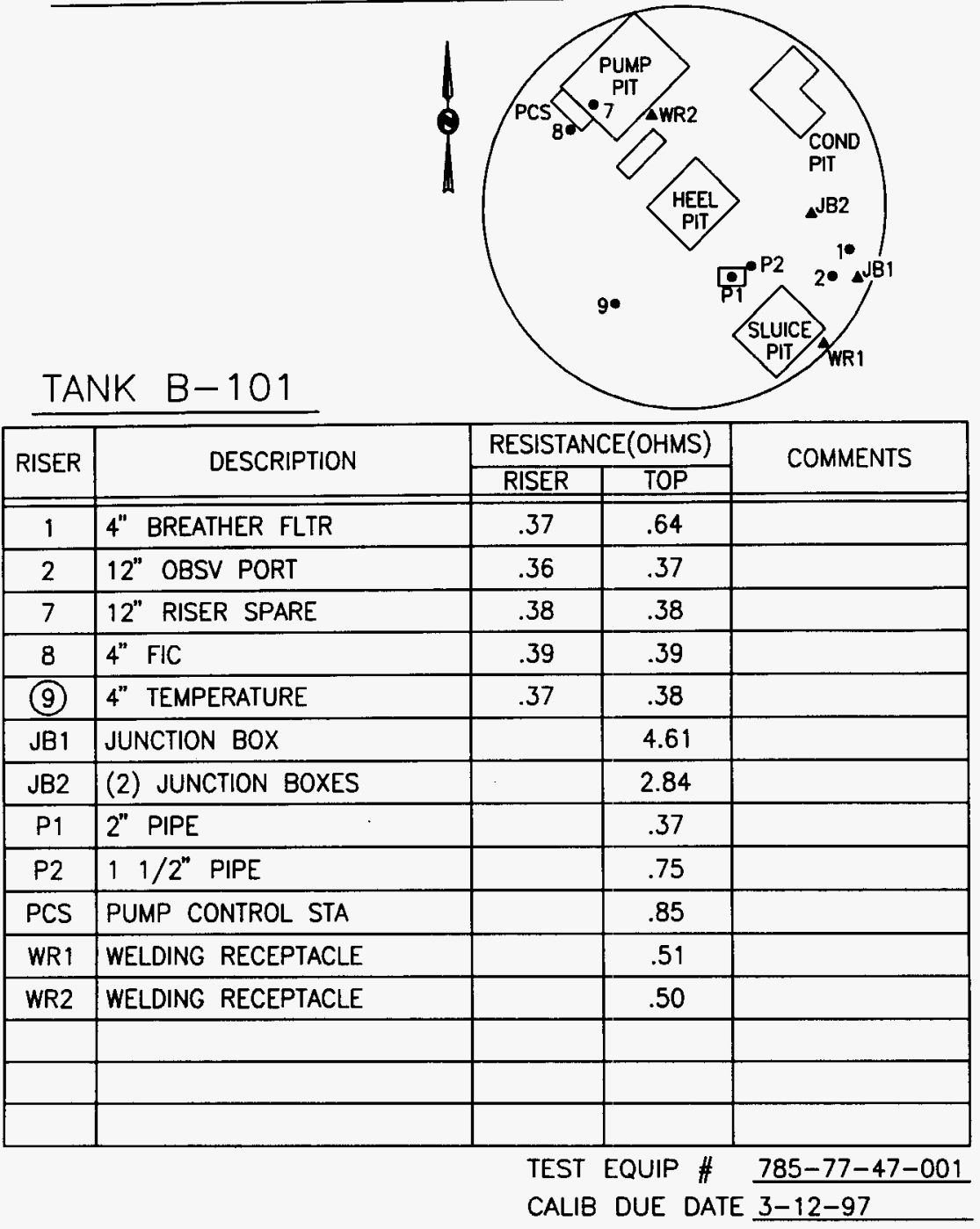


WHC-SD-WM-TR-034, REV. 0

\section{RISER RESISTANCE DATA SHEET}
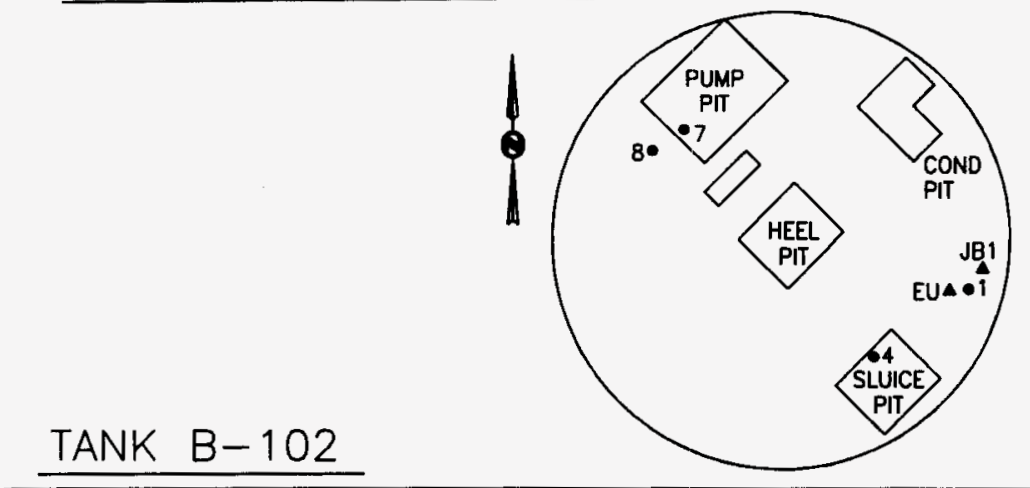

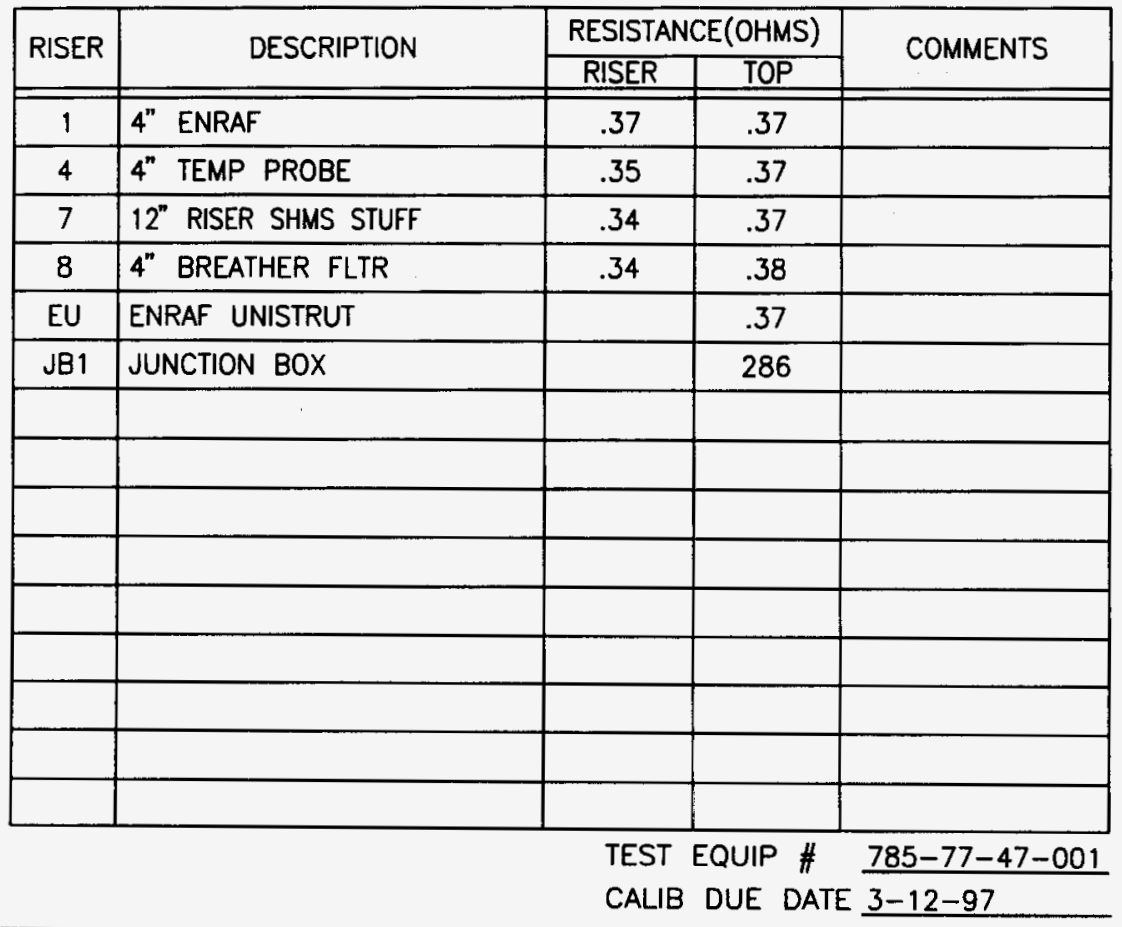


WHC-SD-WM-TR-034, REV. 0

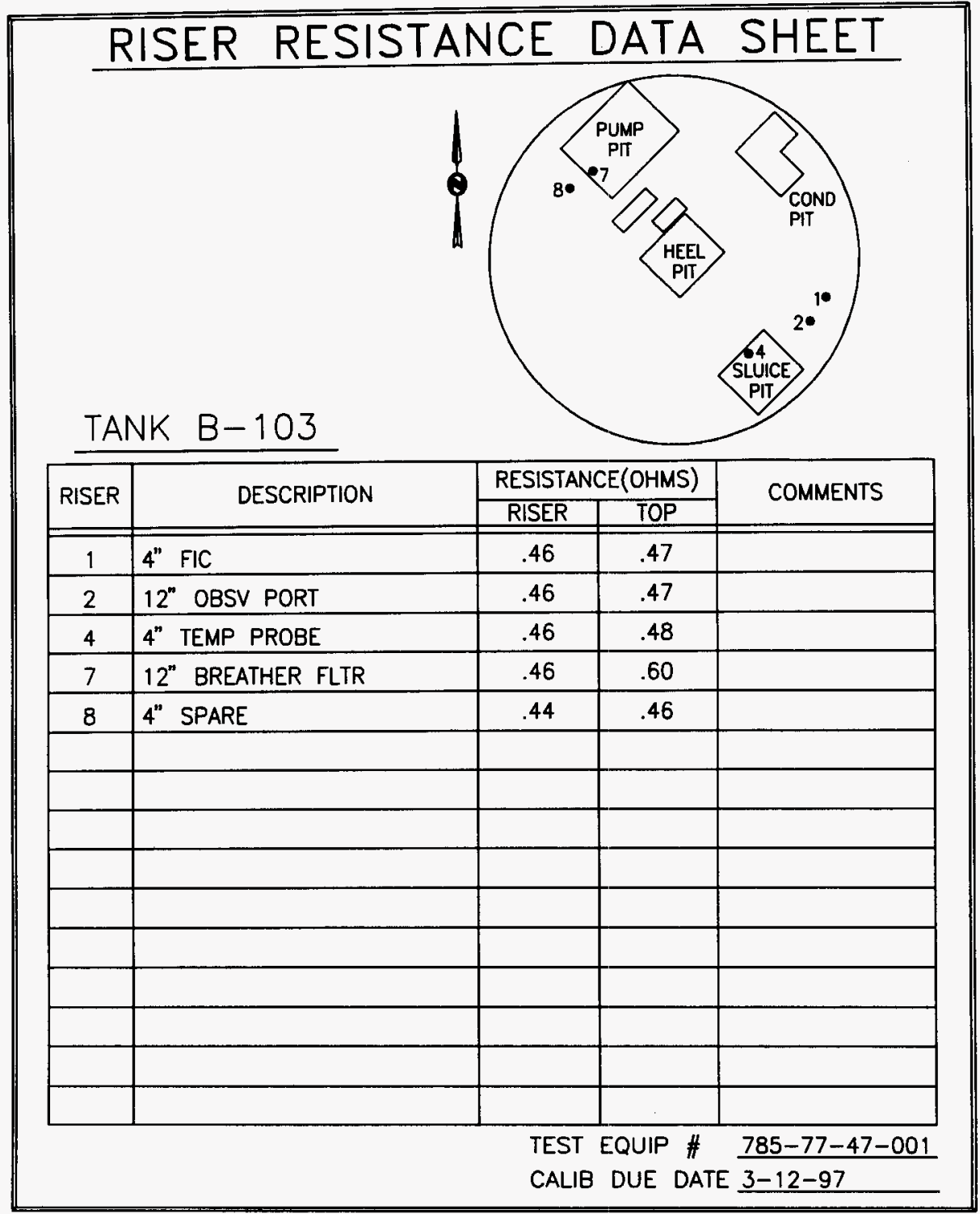




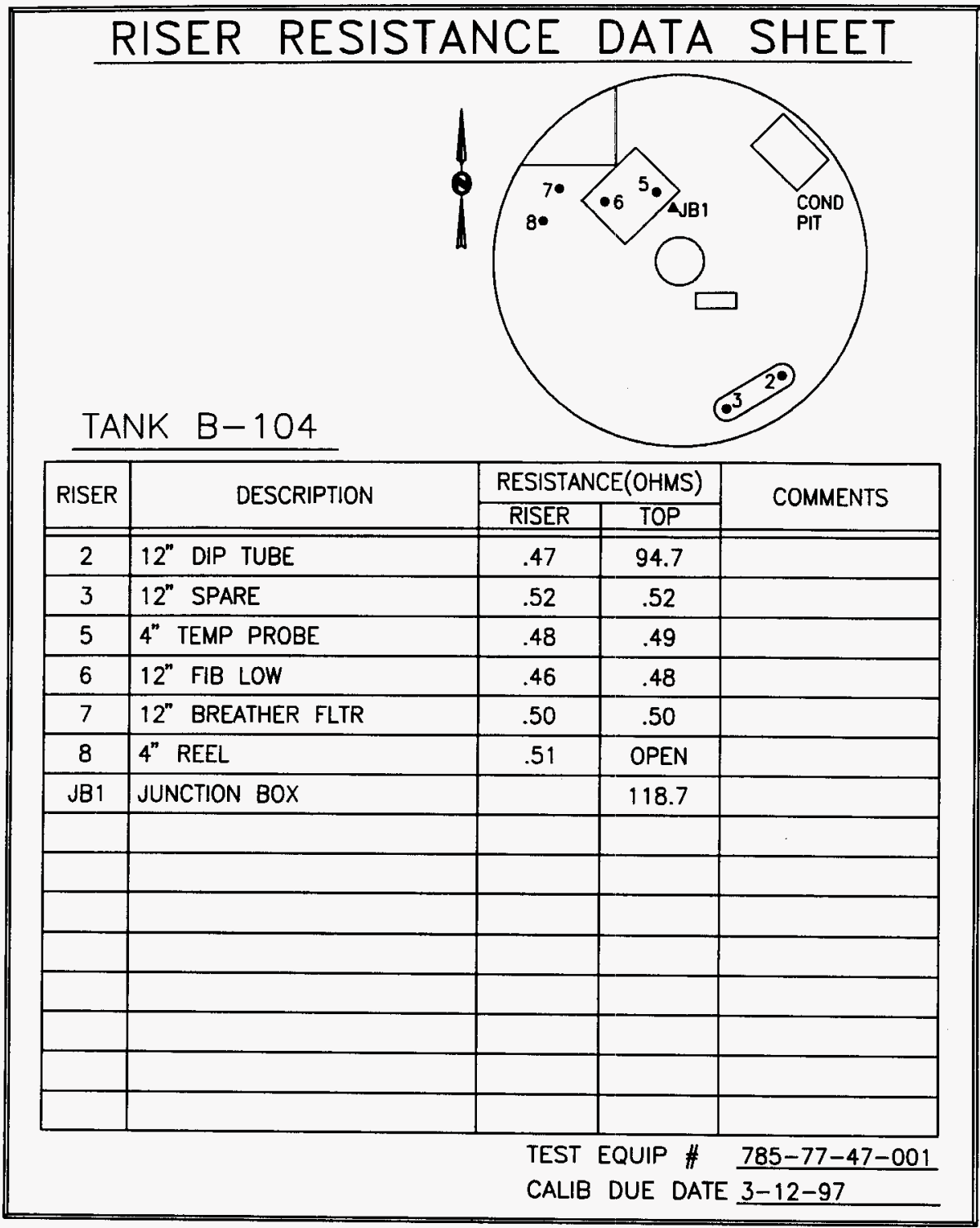


WHC-SD-WM-TR-034, REV. 0

\section{RISER RESISTANCE DATA SHEET}

\begin{tabular}{|c|c|c|c|c|}
\hline \multirow{2}{*}{ RISER } & \multirow{2}{*}{ DESCRIPTION } & \multicolumn{2}{|c|}{ RESISTANCE(OHMS) } & \multirow{2}{*}{ COMMENTS } \\
\hline & & RISER & TOP & \\
\hline 1 & 4" CAISSON & & 128 & NO ACCESS TO RISER \\
\hline 2 & 12" PUMP & 1.02 & 52.8 & \\
\hline 3 & 12" SPARE & 1.03 & 1.03 & \\
\hline 4 & 4" CAISSON & & 188.5 & NO ACCESS TO RISER \\
\hline 5 & 4" FIBERGLASS LOW & 1.01 & & \\
\hline 6 & 12" SPARE & 1.12 & 1.12 & \\
\hline 7 & 12" OBSV PORT & 1.03 & 1.07 & \\
\hline 8 & 4" BREATHER FLTR & 1.02 & 1.02 & \\
\hline (14) & 4" LIQUID LEVEL & 1.18 & 1.18 & \\
\hline (15) & 4" TEMP PROBE & 1.22 & 1.29 & BROKEN \\
\hline JB1 & (2) JUNCTION BOXES & & 128.3 & \\
\hline & & & & \\
\hline & & & & \\
\hline & & & & \\
\hline & & $\begin{array}{l}\text { TES } \\
\text { CAL }\end{array}$ & $\begin{array}{l}\text { QUIP \# } \\
\text { DUE DA }\end{array}$ & $\frac{785-77-47-001}{3-12-97}$ \\
\hline
\end{tabular}




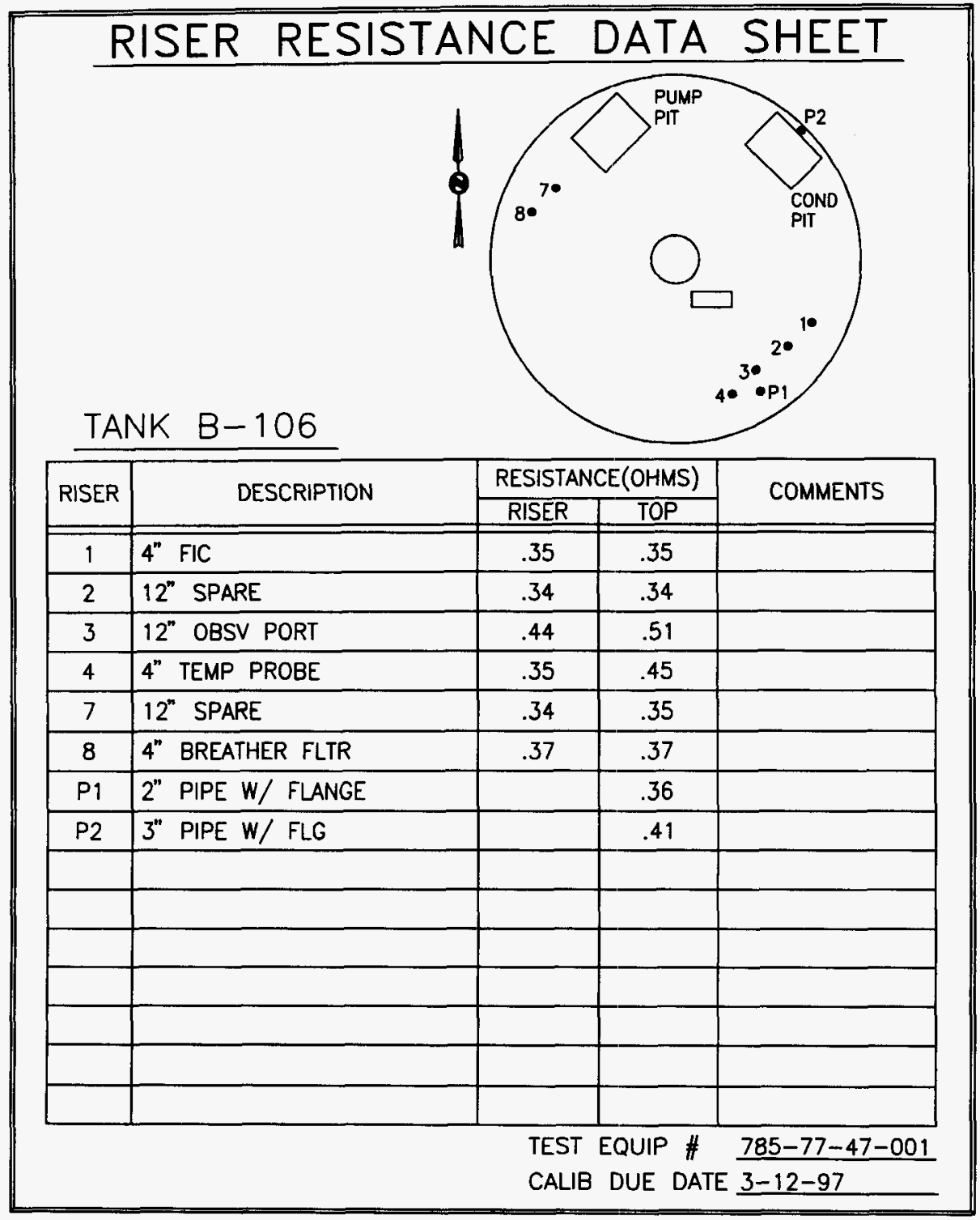


WHC-SD-WM-TR-034, REV. 0

\section{RISER RESISTANCE DATA SHEET}

\begin{tabular}{|c|c|c|c|c|}
\hline \multirow{2}{*}{ RISER } & \multirow{2}{*}{ DESCRIPTION } & \multicolumn{2}{|c|}{ RESISTANCE(OHMS) } & \multirow{2}{*}{ COMMENTS } \\
\hline & & \multicolumn{2}{|c|}{ RISER $\quad$ TOP } & \\
\hline 1 & 4" WEATHER & & & RISER ENCASED \\
\hline 2 & $12^{n}$ OBSV PORT & .49 & .57 & \\
\hline 3 & 12" TEMP PROBE & .44 & .45 & \\
\hline 4 & 4" BREATHER FLTR & .50 & 2.20 & \\
\hline 5 & 4" SPARE & .47 & .47 & \\
\hline 6 & $12^{n}$ SPARE & .49 & 27.3 & \\
\hline 7 & 4" DIP TUBE & .46 & .83 & \\
\hline 8 & 4" REEL & .45 & $8.30(P)$ & PEDESTAL \\
\hline & & & & \\
\hline & & & & \\
\hline & & & & \\
\hline & & & & \\
\hline & & & & \\
\hline & & & & \\
\hline & & & & \\
\hline & & $\begin{array}{l}\text { TES } \\
\text { CAL }\end{array}$ & $\begin{array}{l}\text { QUIP \# } \\
\text { DUE DAT }\end{array}$ & $\frac{785-77-47-0}{3-12-97}$ \\
\hline
\end{tabular}


WHC-SD-WM-TR-034, REV. 0

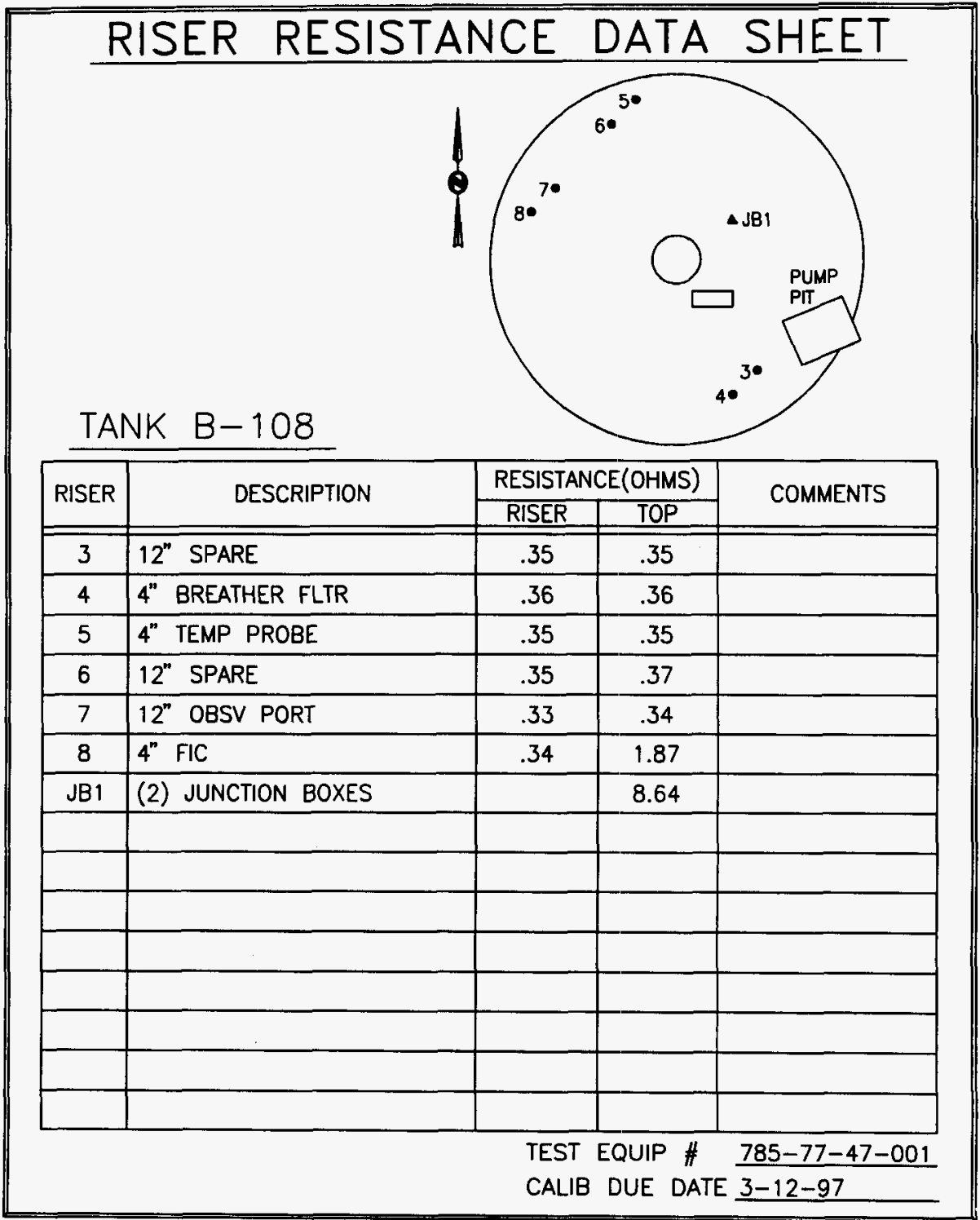


WHC-SD-WM-TR-034, REV. 0

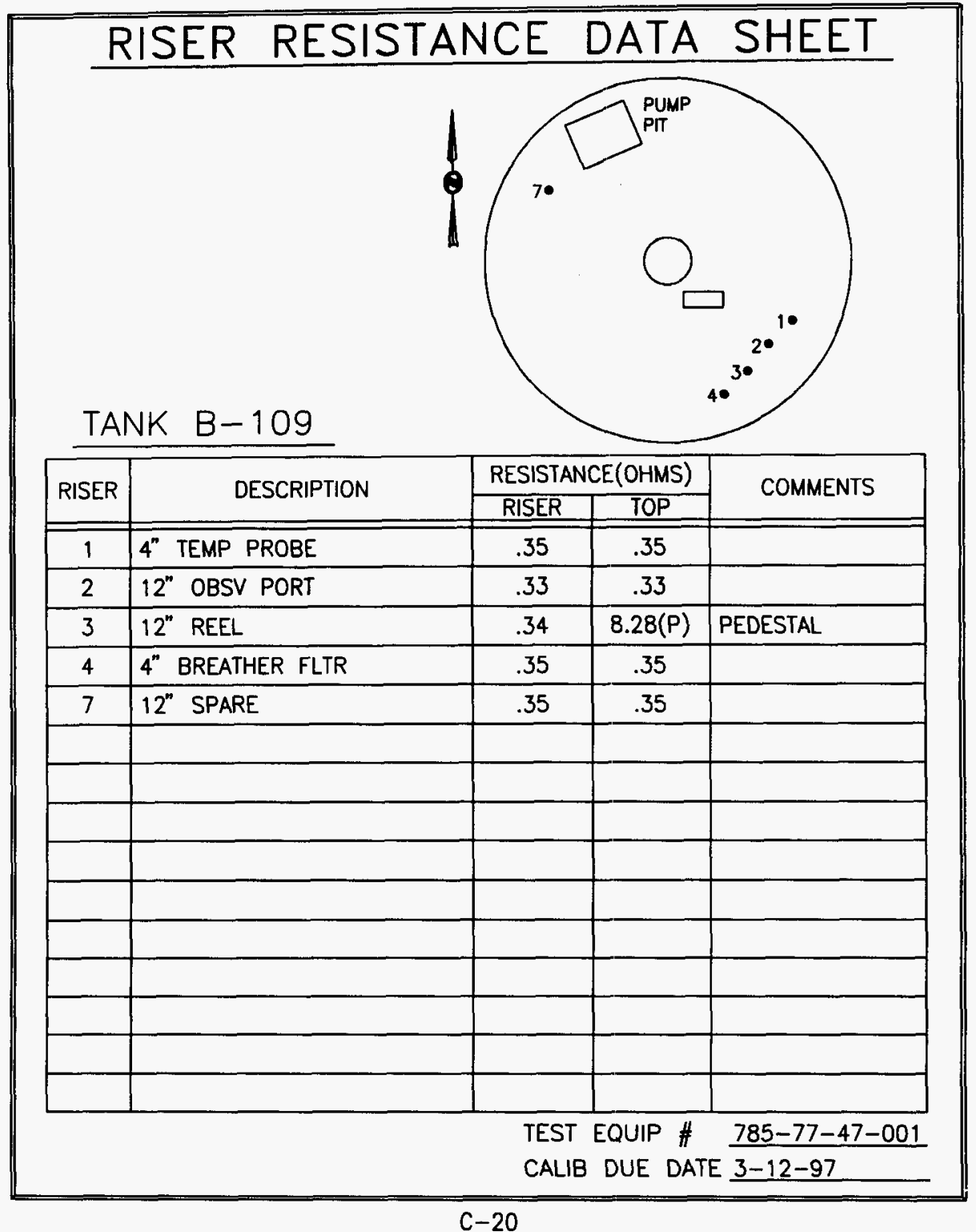


WHC-SD-WM-TR-034, REV. 0

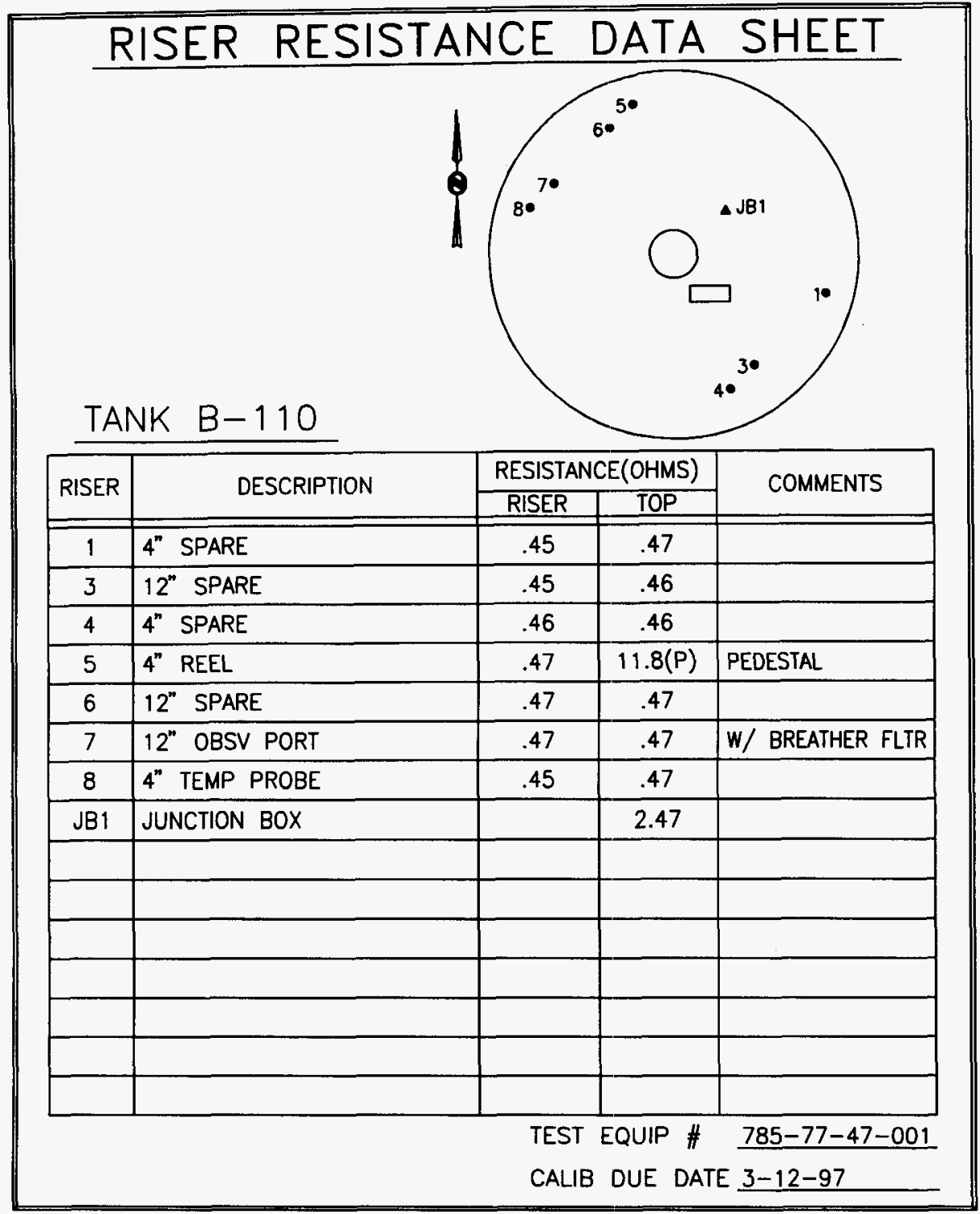


WHC-SD-WM-TR-034, REV. 0

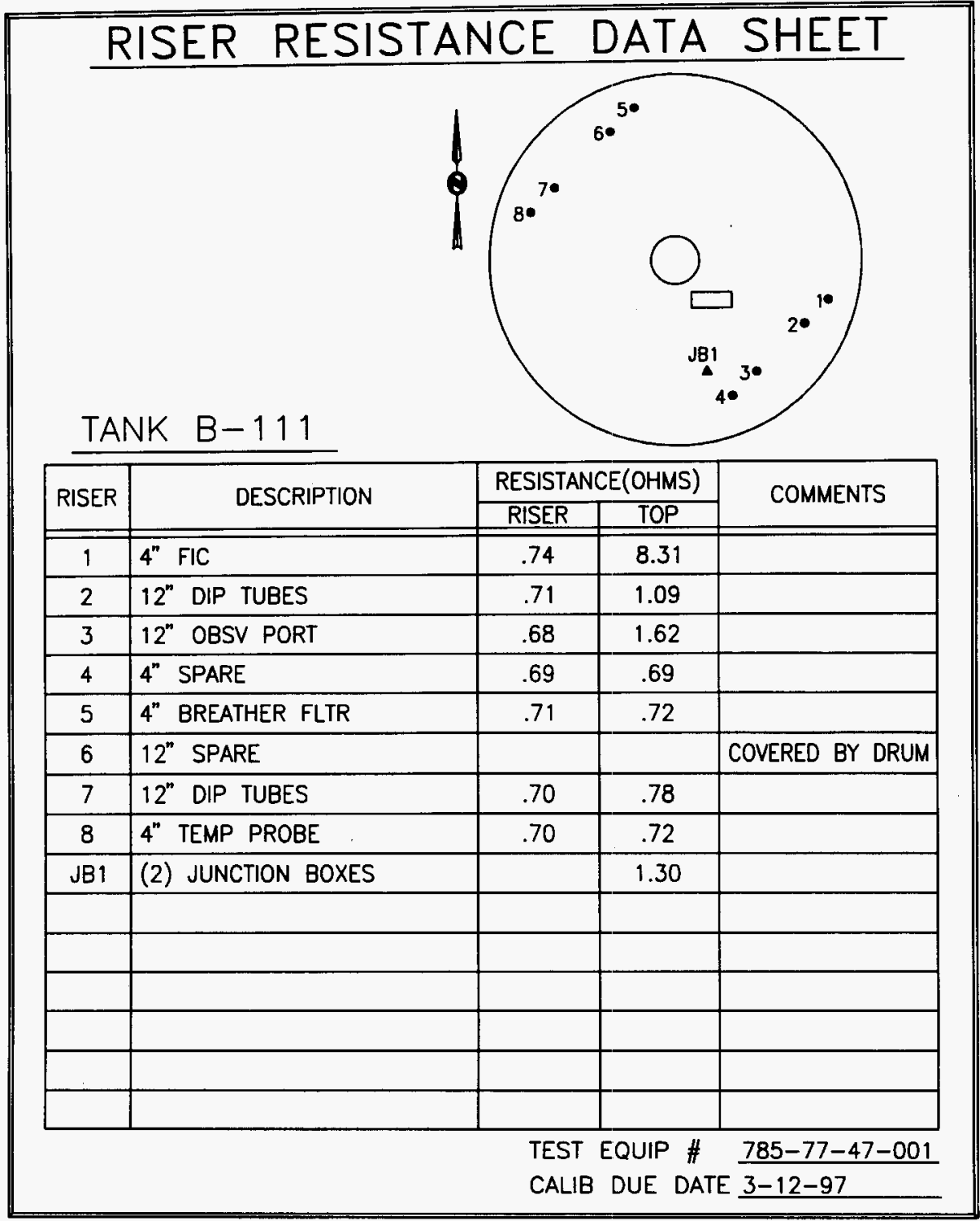


WHC-SD-WM-TR-034, REV. 0

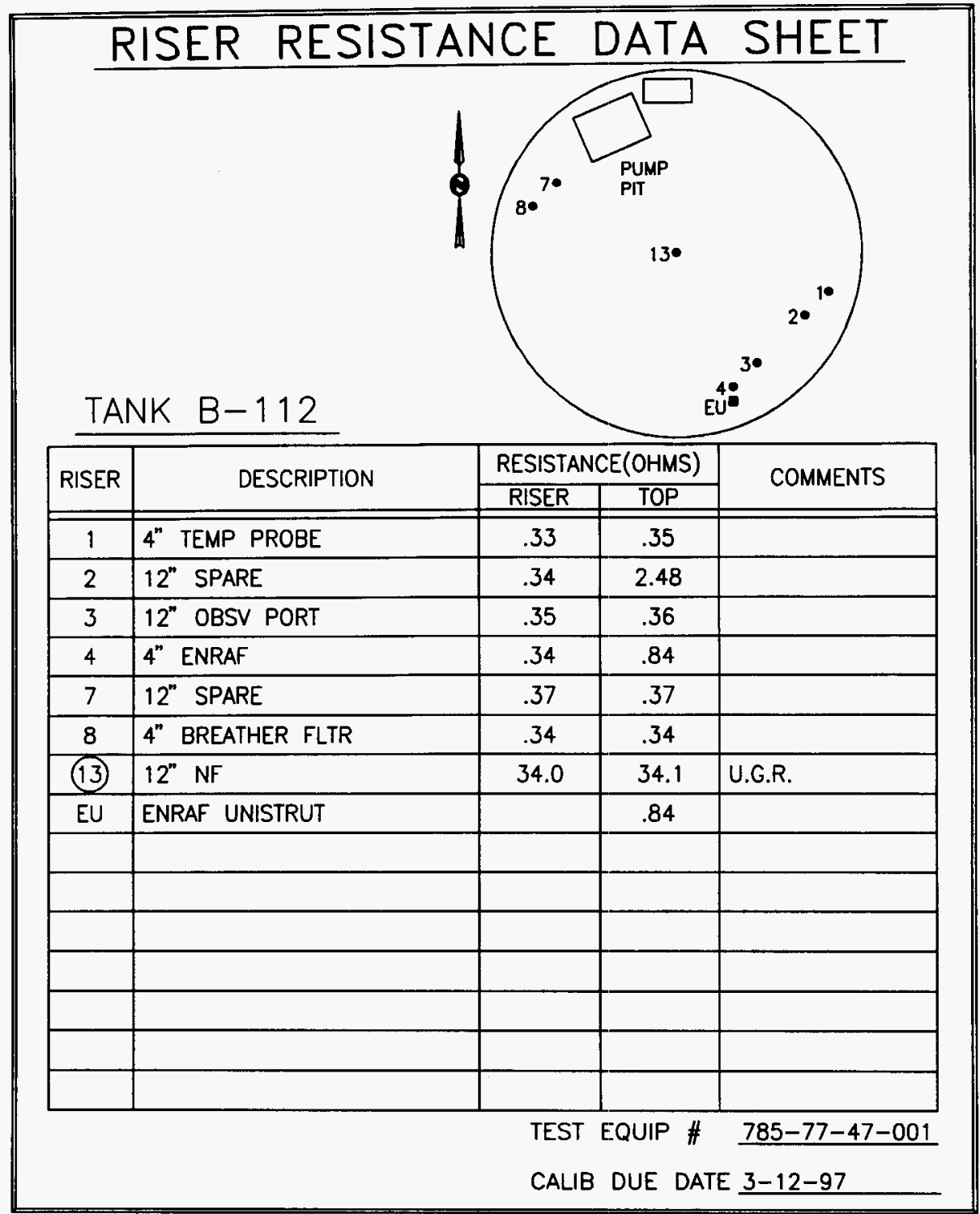


WHC-SD-WM-TR-034, REV. O

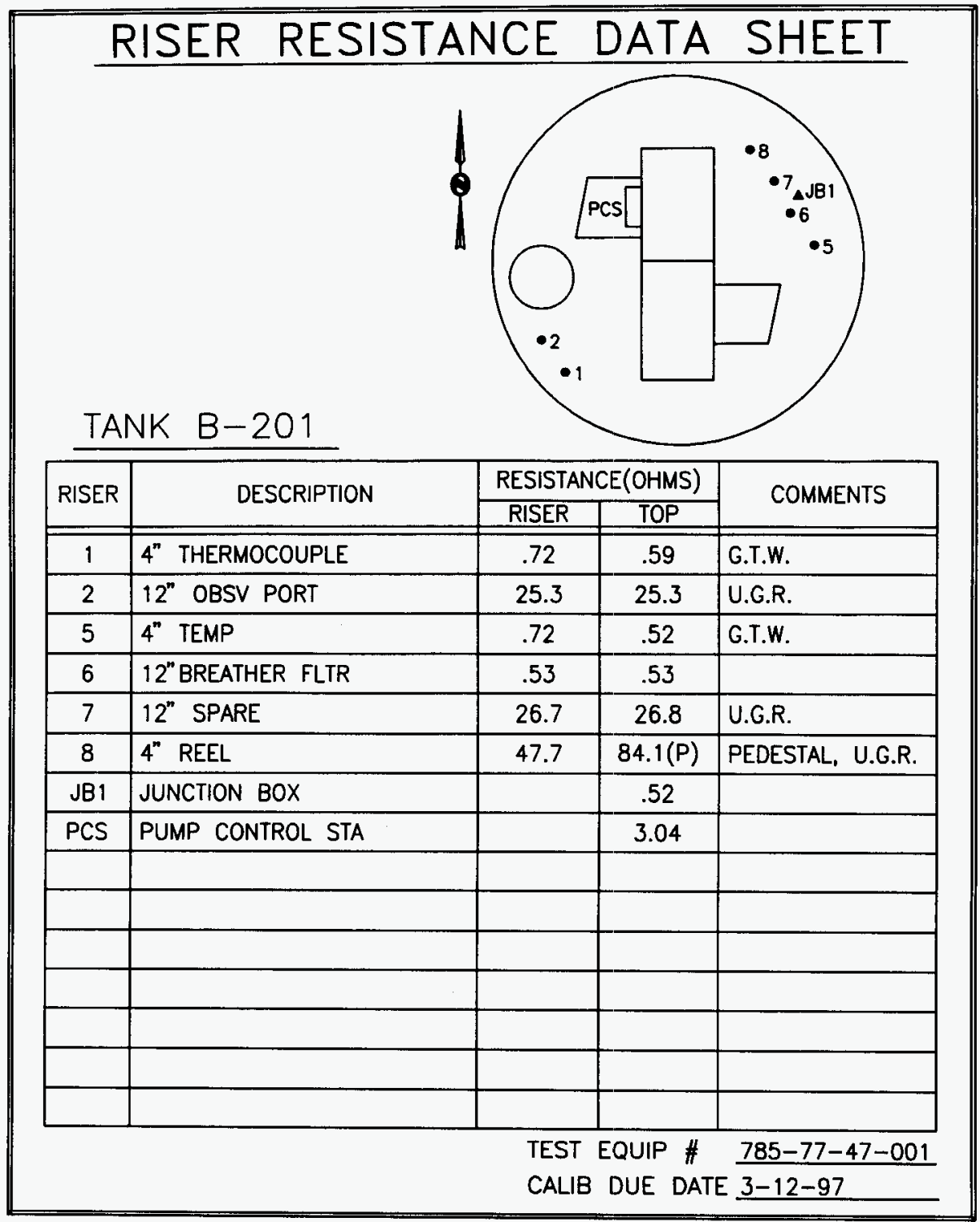




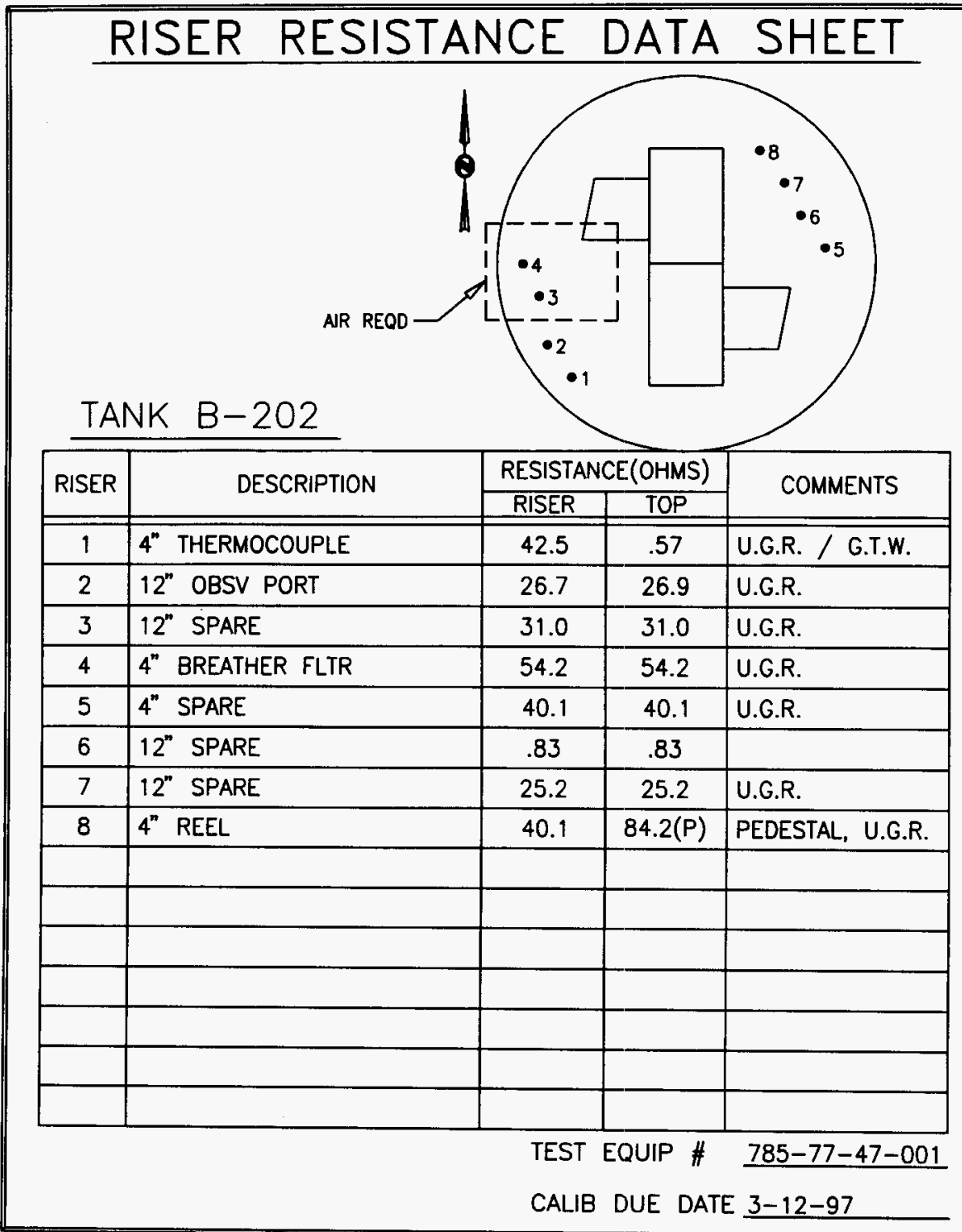


WHC-SD-WM-TR-034, REV. 0

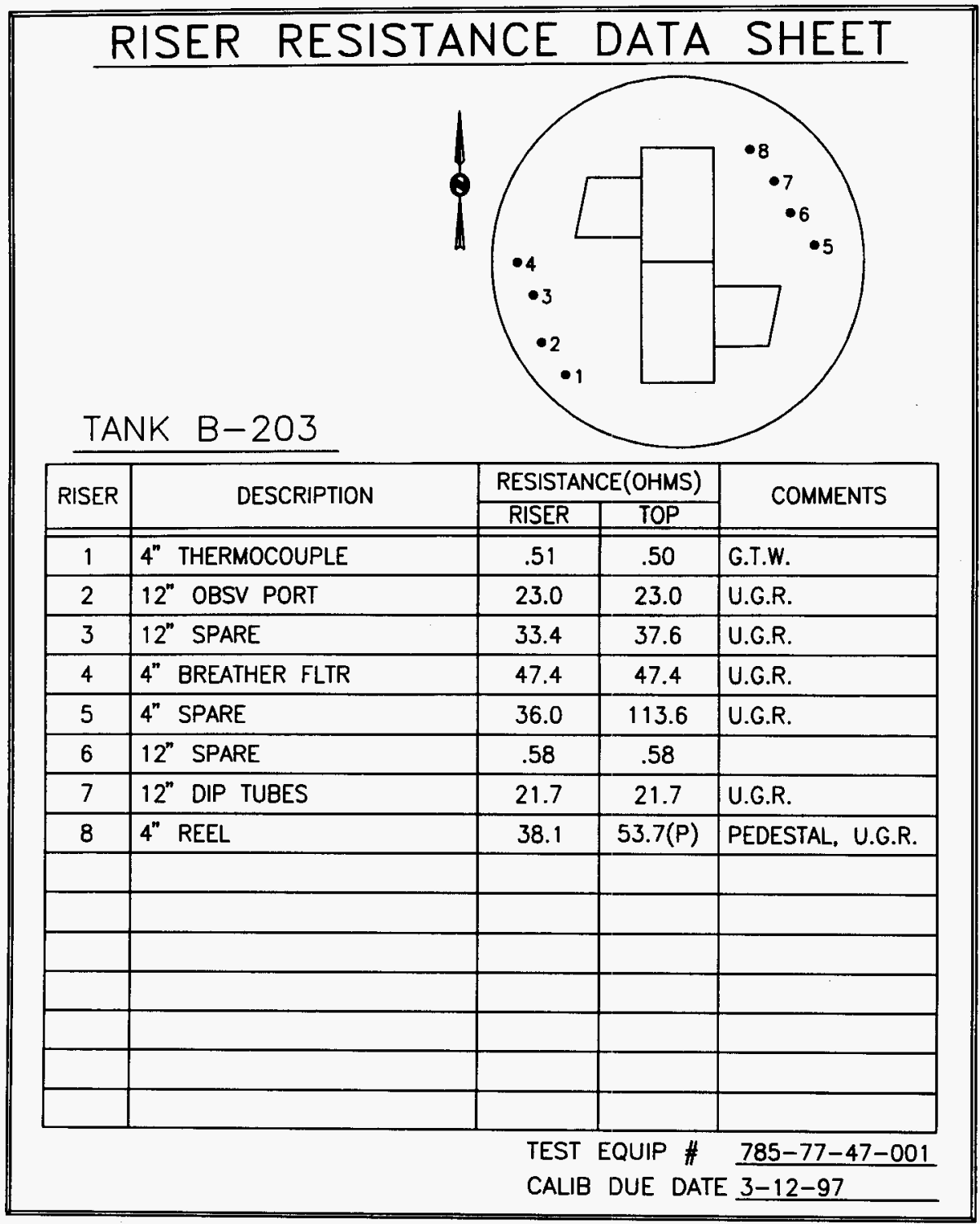


WHC-SD-WM-TR-034, REV. 0

\section{RISER RESISTANCE DATA SHEET}

TANK B-204

\begin{tabular}{|c|c|c|c|c|}
\hline \multirow{2}{*}{ RISER } & \multirow{2}{*}{ DESCRIPTION } & \multicolumn{2}{|c|}{ RESISTANCE(OHMS) } & \multirow{2}{*}{ COMMENTS } \\
\hline & & RISER & TOP & \\
\hline 1 & $4^{n} \quad$ THERMOCOUPLE & .61 & .53 & G.T.W. \\
\hline 2 & $12^{n}$ OBSV PORT & 23.0 & 23.0 & U.G.R. \\
\hline 3 & $12^{\prime \prime}$ SPARE & 24.9 & 25.0 & U.G.R. \\
\hline 4 & $4^{\prime \prime}$ BREATHER FLTR & .59. & .59 & \\
\hline 5 & $4^{n}$ SPARE & 30.8 & $3.5 \mathrm{~K}$ & U.G.R. \\
\hline 6 & $12^{n}$ SPARE & 24.0 & 24.0 & U.G.R. \\
\hline 7 & $12^{n}$ SPARE & 22.4 & 22.4 & U.G.R. \\
\hline 8 & 4" REEL & 39.8 & $83.0(P)$ & PEDESTAL, U.G.R. \\
\hline & & & & \\
\hline & & & & \\
\hline & & & & \\
\hline & & & & \\
\hline & & & & \\
\hline & & & & \\
\hline & & $\begin{array}{l}\text { TEST } \\
\text { CALI }\end{array}$ & $\begin{array}{l}\text { QUIP \# } \\
\text { DUE DAT }\end{array}$ & $\begin{array}{l}785-77-47-001 \\
3-12-97 \\
\end{array}$ \\
\hline
\end{tabular}


WHC-SD-WM-TR-034, REV. 0

RISER RESISTANCE DATA SHEET

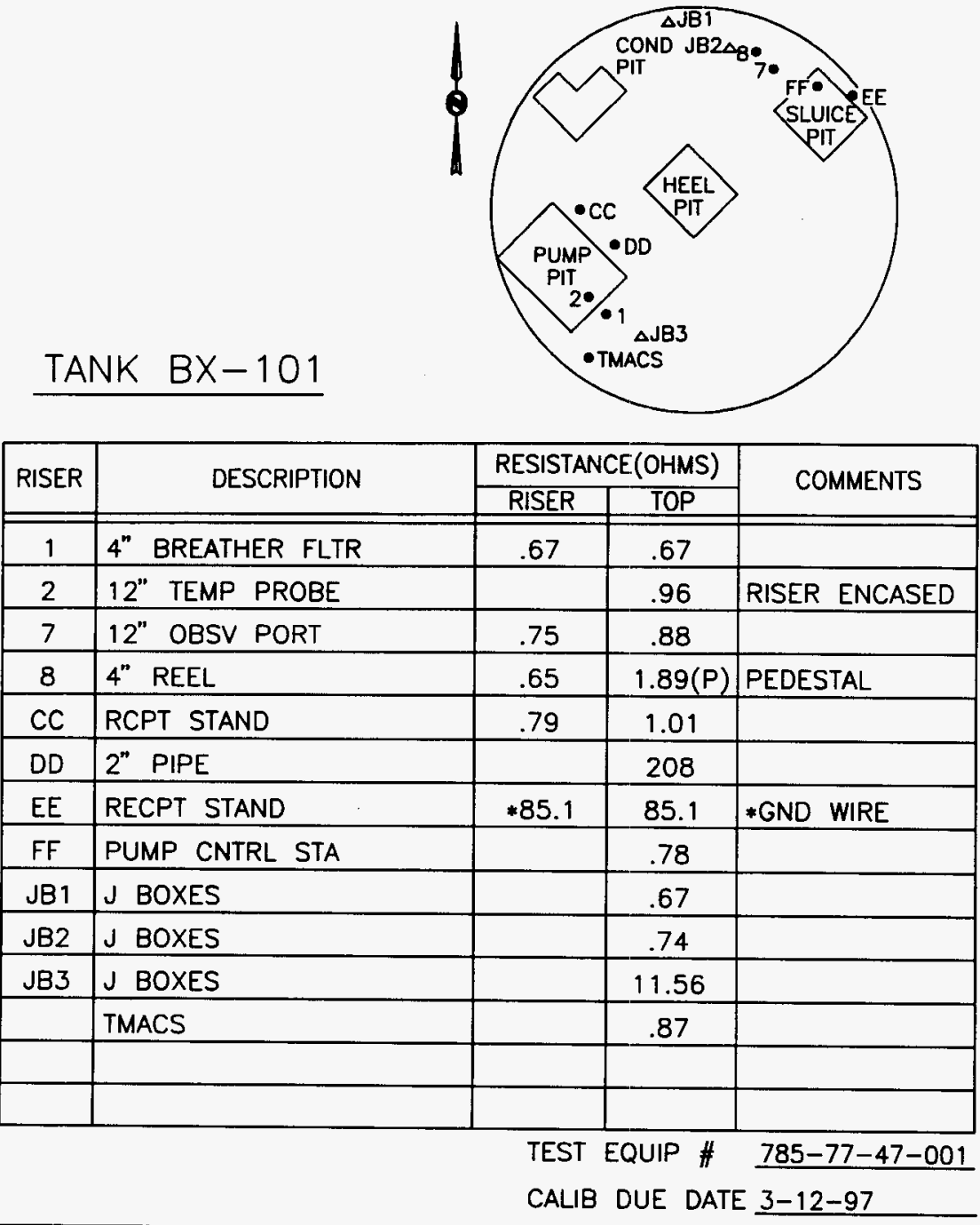




\section{RISER RESISTANCE DATA SHEET}

TANK BX-102
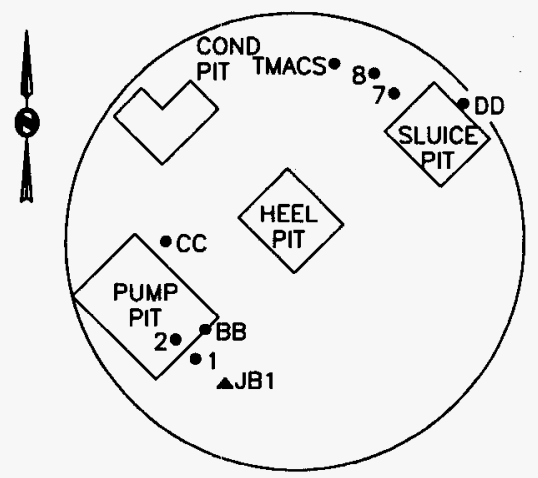

\begin{tabular}{|c|c|c|c|c|}
\hline \multirow{2}{*}{ RISER } & \multirow{2}{*}{ DESCRIPTION } & \multicolumn{2}{|c|}{ RESISTANCE(OHMS) } & \multirow{2}{*}{ COMMENTS } \\
\hline & & RISER & TOP & \\
\hline 1 & 4" TEMP PROBE & .65 & .66 & \\
\hline 2 & 12" REEL & .66 & $.66(P)$ & PEDESTAL \\
\hline 7 & $\begin{array}{ll}12 " & \text { OBSV PORT } \\
\text { AND BREATHER }\end{array}$ & .71 & .84 & \\
\hline 8 & 4" TEMP PROBE & .65 & .65 & \\
\hline $\mathrm{BB}$ & METAL ANGLE & & 80.4 & \\
\hline $\mathrm{CC}$ & RCPT STAND & $\star .77$ & .84 & *GND WIRE \\
\hline DD & RECPT STAND & $* .79$ & 7.9 & *GND WIRE \\
\hline \multirow[t]{2}{*}{ JB1 } & $J$ BOX & & 28.6 & \\
\hline & TMACS & & .73 & \\
\hline & & & & \\
\hline & & & & \\
\hline & . & & & \\
\hline & & & & \\
\hline
\end{tabular}

TEST EQUIP \# 785-77-47-001

CALIB DUE DATE 3-12-97 
WHC-SD-WM-TR-034, REV. 0

\section{RISER RESISTANCE DATA SHEET}

TANK BX-103

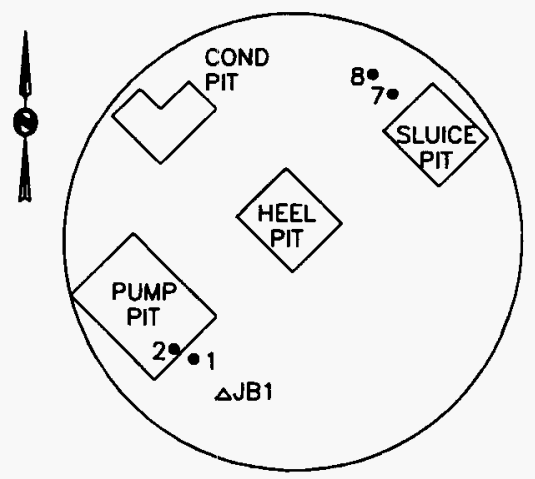

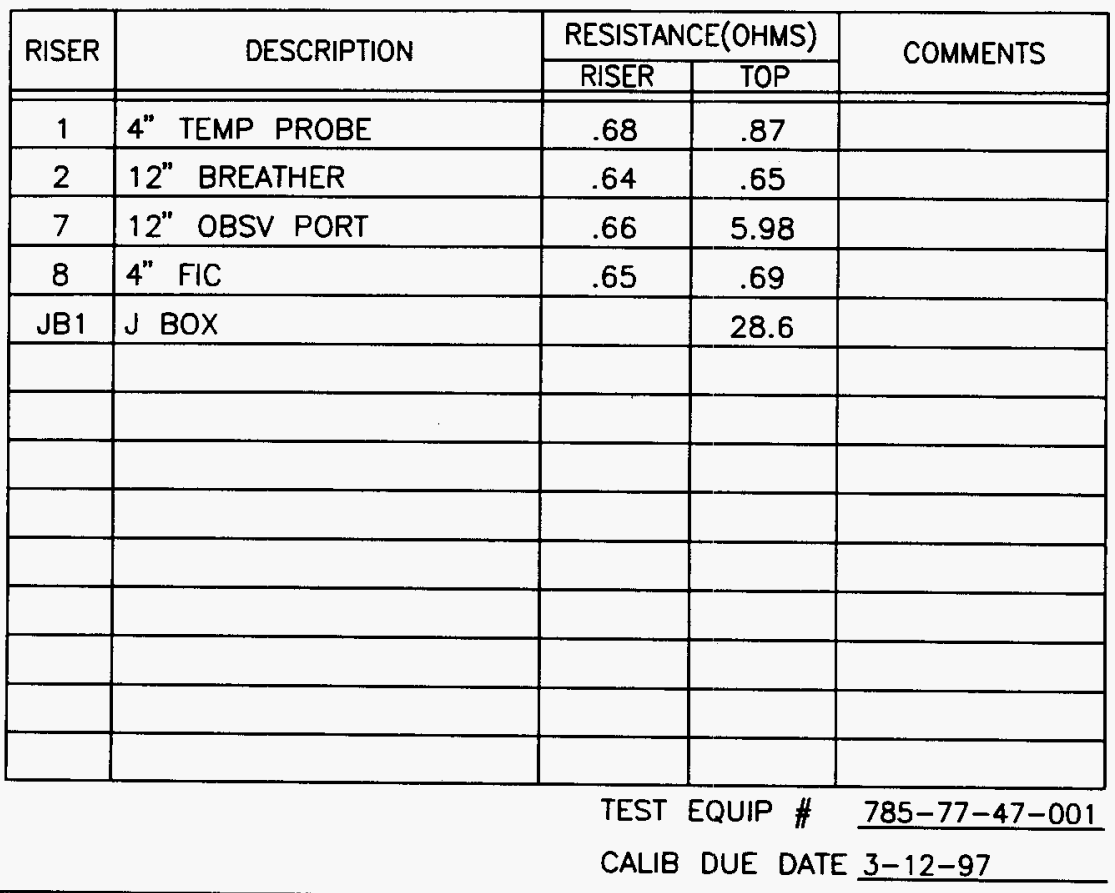


WHC-SD-WM-TR-034, REV. 0

RISER RESISTANCE DATA SHEET

TANK BX-104

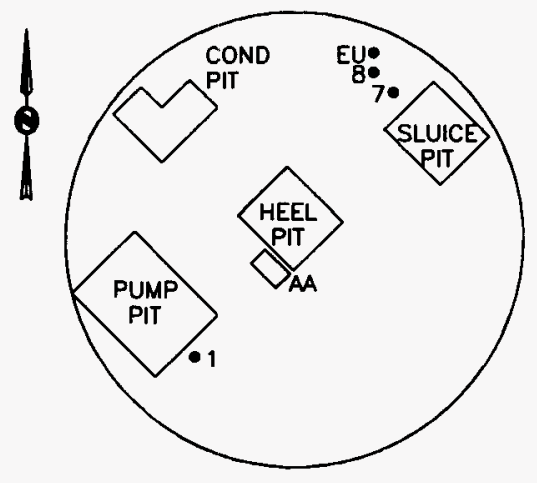

\begin{tabular}{|c|l|c|c|c|}
\hline \multirow{2}{*}{ RISER } & \multirow{2}{*}{ DESCRIPTION } & \multicolumn{2}{c|}{ RESISTANCE(OHMS) } & \multirow{2}{*}{ COMMENTS } \\
\cline { 3 - 4 } & & RISER & TOP & \\
\hline \hline 1 & $4 "$ BREATHER FLTR & .68 & .89 & \\
\hline 7 & $12^{\prime \prime}$ OBSV PORT & .70 & .70 & \\
\hline 8 & $4 "$ ENRAF & .67 & .73 & \\
\hline AA & PUMP CNTRL RACK & & .79 & \\
\hline EU & ENRAF UNISTRUT & & .79 & \\
\hline & & & & \\
\hline & & & & \\
\hline & & & & \\
\hline & & & & \\
\hline & & & & \\
\hline & & & & \\
\hline & & & & \\
\hline & & & & \\
\hline & & & & \\
\hline
\end{tabular}

TEST EQUIP \# 785-77-47-001

CALIB DUE DATE 3-12-97 
WHC-SD-WM-TR-034, REV. 0

\section{RISER RESISTANCE DATA SHEET}

TANK BX-105

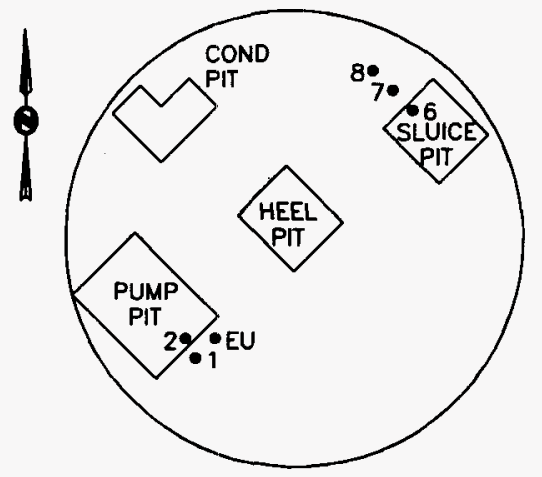

\begin{tabular}{|c|c|c|c|c|}
\hline \multirow{2}{*}{ RISER } & \multirow{2}{*}{ DESCRIPTION } & \multicolumn{2}{|c|}{ RESISTANCE(OHMS) } & \multirow{2}{*}{ COMMENTS } \\
\hline & & RISER & TOP & \\
\hline 1 & 4" ENRAF & .67 & .79 & \\
\hline 2 & 12" SPARE & .67 & .70 & \\
\hline 6 & 12" SPARE & .79 & .79 & \\
\hline 7 & 12" TEMP PROBE & .67 & .87 & \\
\hline 8 & 4" BREATHER FLTR & .68 & .75 & \\
\hline EU & ENRAF UNISTRUT & & .77 & \\
\hline & & & & \\
\hline & & & & \\
\hline & & & & \\
\hline & & & & \\
\hline & & & & \\
\hline & & & & \\
\hline & & & & \\
\hline & & & & \\
\hline & & TES & UIP \# & $35-77-47-001$ \\
\hline & & CALI & UE DA & $-12-97$ \\
\hline
\end{tabular}


WHC-SD-WM-TR-034, REV. 0

\section{RISER RESISTANCE DATA SHEET}

TANK BX-106

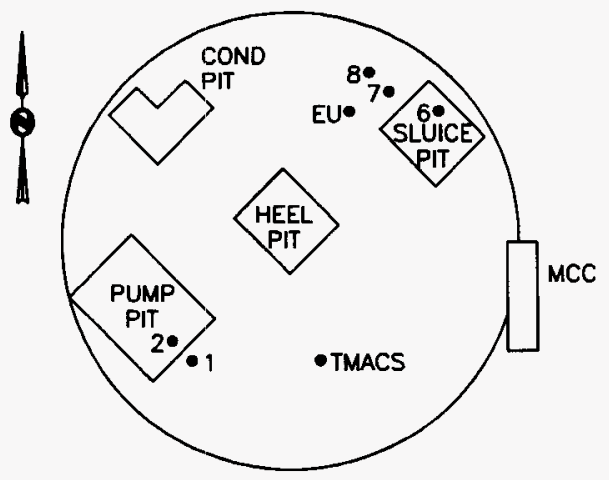

\begin{tabular}{|c|c|c|c|c|}
\hline \multirow{2}{*}{ RISER } & \multirow{2}{*}{ DESCRIPTION } & \multicolumn{2}{|c|}{ RESISTANCE(OHMS) } & \multirow{2}{*}{ COMMENTS } \\
\hline & & RISER & TOP & \\
\hline 1 & 4" THERMO TREE & .69 & .70 & \\
\hline 2 & BREATHER & .71 & 46.3 & \\
\hline 6 & 12" SPARE & .67 & .73 & \\
\hline 7 & 12" TEMP PROBE & .68 & .92 & \\
\hline 8 & 4" ENRAF & .68 & .86 & \\
\hline EU & ENRAF UNISTRUT & & .84 & \\
\hline \multirow[t]{2}{*}{$\mathrm{MCC}$} & MCC & $* .68$ & & *GND PAD \\
\hline & TMACS & & .71 & \\
\hline & & & & \\
\hline & & & & \\
\hline & & & & \\
\hline & & & & \\
\hline & & & & \\
\hline & & & & \\
\hline & & TES & UIP \# & $785-77-47-001$ \\
\hline & & CAL & UE DAT & $3-12-97$ \\
\hline
\end{tabular}


WHC-SD-WM-TR-034, REV. 0

\section{RISER RESISTANCE DATA SHEET}

TANK BX-107

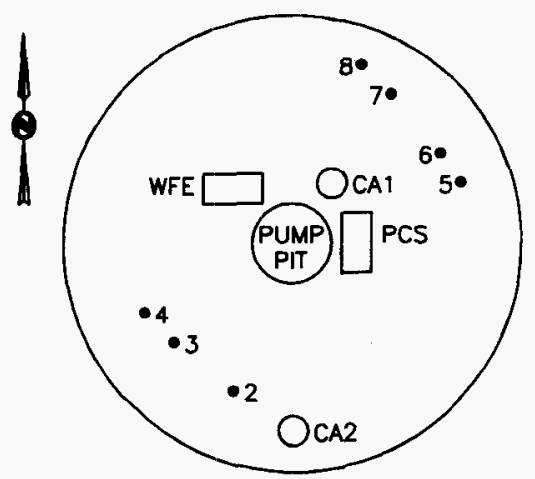

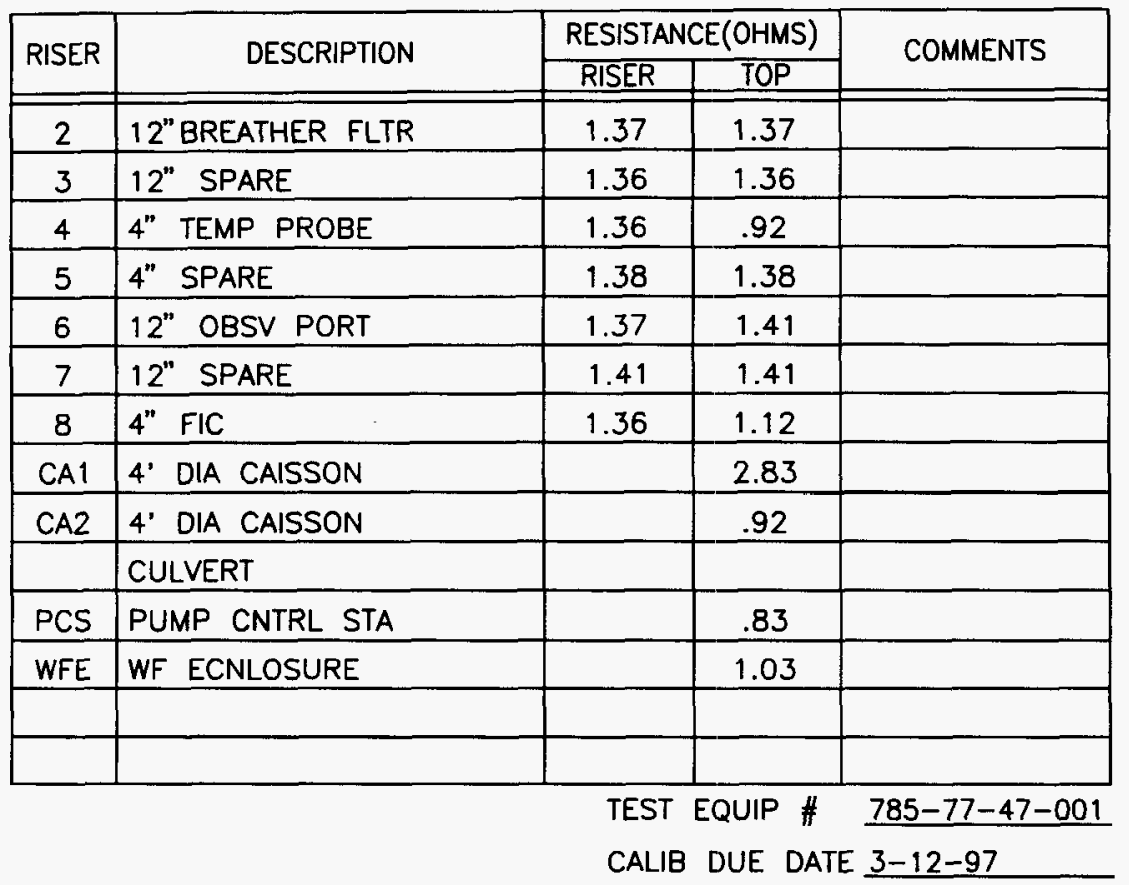




\section{RISER RESISTANCE DATA SHEET}

TANK BX-108

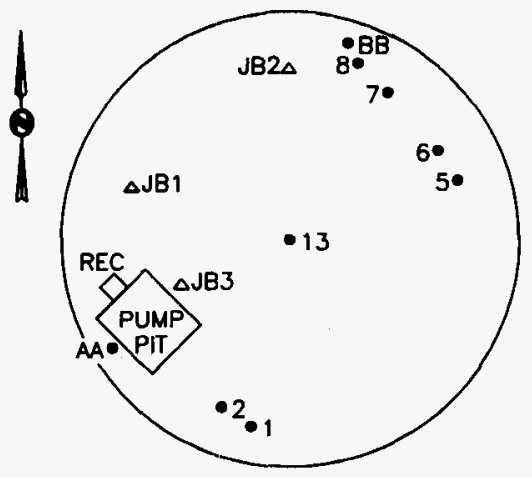

\begin{tabular}{|c|c|c|c|c|}
\hline \multirow{2}{*}{ RISER } & \multirow{2}{*}{ DESCRIPTION } & \multicolumn{2}{|c|}{ RESISTANCE(OHMS) } & \multirow{2}{*}{ COMMENTS } \\
\hline & & RISER & TOP & \\
\hline 1 & 4" BREATHER FLTR & 1.67 & 1.67 & \\
\hline 2 & 12" SPARE & 1.70 & 1.77 & \\
\hline 5 & 4" TEMP PROBE & 1.75 & 1.69 & \\
\hline 6 & 12" OBSV PORT & 1.68 & 1.82 & \\
\hline 7 & 12" REEL & 1.73 & 581 & \\
\hline 8 & 4" SPARE & 1.67 & OPEN & \\
\hline (13) & 12" SPARE & 5.56 & 5.57 & UGR \\
\hline$A A$ & HEAT TRACE CNTRL & & 2.63 & \\
\hline BB & FIC & & & \\
\hline JB1 & $J$ BOX & & & \\
\hline JB2 & J BOX & & 1.02 & \\
\hline $\mathrm{JB3}$ & PHOSPHORIC J BOX & & & \\
\hline REC & RECEPTACLE & & 238 & \\
\hline & & & & \\
\hline & & $\begin{array}{l}\text { TES } \\
\text { CAL }\end{array}$ & $\begin{array}{l}\text { QUIP \# } \\
\text { UE DA }\end{array}$ & $\begin{array}{l}785-77-47-001 \\
3-12-97\end{array}$ \\
\hline
\end{tabular}


WHC-SD-WM-TR-034, REV. 0

\section{RISER RESISTANCE DATA SHEET}

TANK BX-109

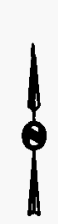

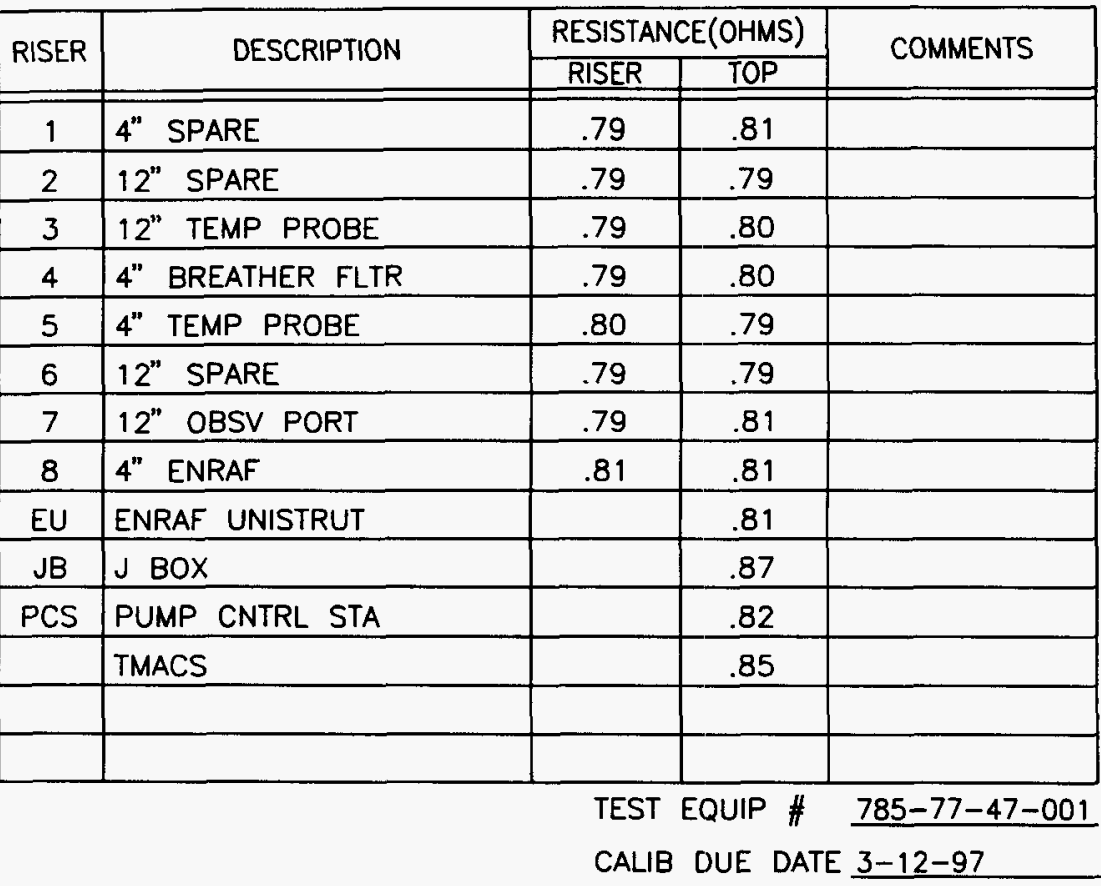


WHC-SD-WM-TR-034, REV. 0

\section{RISER RESISTANCE DATA SHEET}

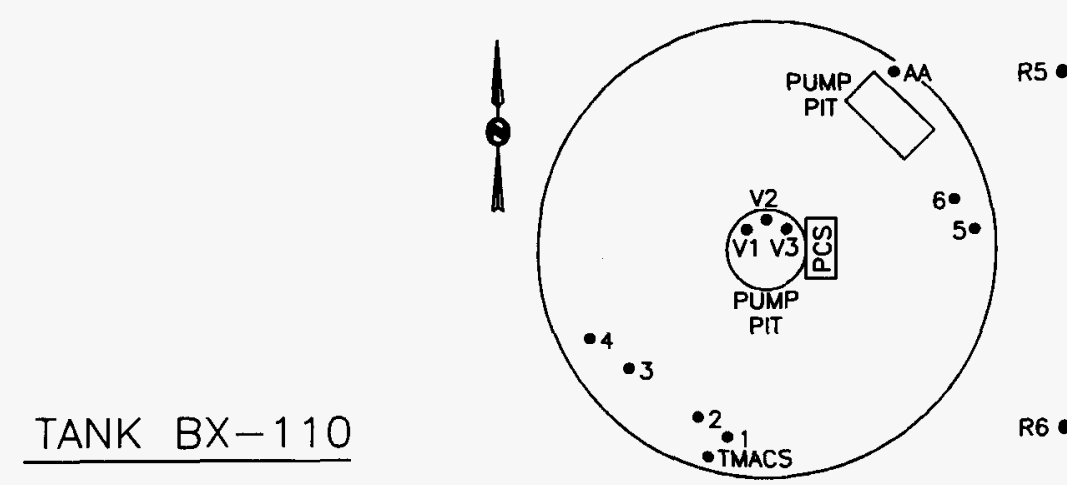

\begin{tabular}{|c|l|c|c|c|}
\hline \multirow{2}{*}{ RISER } & \multicolumn{1}{|c|}{ DESCRIPIION } & \multicolumn{2}{c|}{ RESISTANCE(OHMS) } & \multirow{2}{*}{ COMMENTS } \\
\cline { 3 - 4 } & & RISER & TOP & \\
\hline \hline 1 & $4 "$ TEMP PROBE & .83 & .83 & \\
\hline 2 & $12^{\prime \prime}$ REEL & .87 & $* 96.7$ & $*$ *PEDESTAL \\
\hline 3 & $12 "$ OBSV PORT & .86 & .85 & \\
\hline 4 & $4 "$ BREATHER FLTR & .84 & .84 & \\
\hline 6 & $12 "$ SPARE & .84 & .85 & \\
\hline 5 & $4 "$ SLUDGE MEAS & .82 & .96 & \\
\hline R5 & $4 "$ PIPE RISER & 3.67 & 3.67 & \\
\hline R6 & $4 "$ PIPE RISER & 3.67 & 3.67 & \\
\hline AA & PUMP CONTROLLER & & .84 & \\
\hline PCS & PUMP CNTRL STA & & .88 & \\
\hline & TMACS & & .99 & \\
\hline V1 & VALVE HANOLE & & OPEN & \\
\hline V2 & VALVE HANDLE & & OPEN & \\
\hline V3 & VALVE HANDLE & & 34.5 & \\
\hline
\end{tabular}

TEST EQUIP \# 785-77-47-001

CALIB DUE DATE 3-12-97 
WHC-SD-WM-TR-034, REV. 0

\section{RISER RESISTANCE DATA SHEET}

TANK BX-111
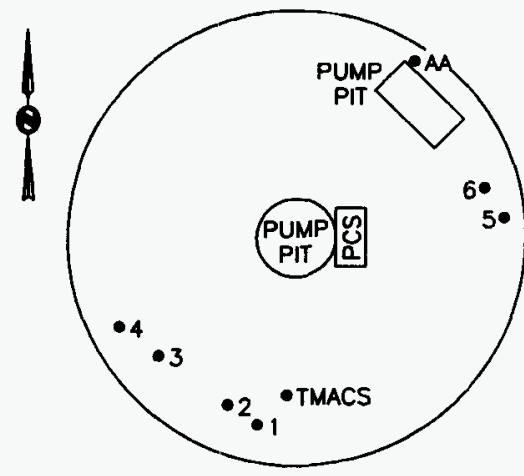

R3•

$\mathrm{R} 4 \bullet$

\begin{tabular}{|c|l|c|c|c|}
\hline \multirow{2}{*}{ RISER } & \multirow{2}{*}{ DESCRIPTION } & \multicolumn{2}{c|}{ RESISTANCE(OHMS) } & \multirow{2}{*}{ COMMENTS } \\
\cline { 3 - 4 } & & RISER & TOP & \\
\hline \hline 1 & $4 "$ & .83 & .83 & \\
\hline 2 & $12 "$ TEMP PROBE & .82 & .97 & \\
\hline 3 & $12 "$ OBSV PORT & .83 & 9330 & \\
\hline 4 & $4 "$ BREATHER FLTR & .82 & .91 & \\
\hline 5 & $4 "$ LOW & .82 & & \\
\hline 6 & $12 "$ SPARE & .82 & .82 & \\
\hline R3 & $4 "$ PIPE RISER & 3.63 & 3.63 & \\
\hline R4 & $4 "$ PIPE RISER & 3.64 & 3.72 & \\
\hline AA & PUMP CONTROLLER & & .83 & \\
\hline PCS & PUMP CNTRL STA & & .89 & \\
\hline & TMACS & & .94 & \\
\hline & & & & \\
\hline & & & & \\
\hline & & & & \\
\hline
\end{tabular}

TEST EQUIP \# 785-77-47-001

CALIB DUE DATE 3-12-97 
WHC-SD-WM-TR-034, REV. 0

\section{RISER RESISTANCE DATA SHEET}

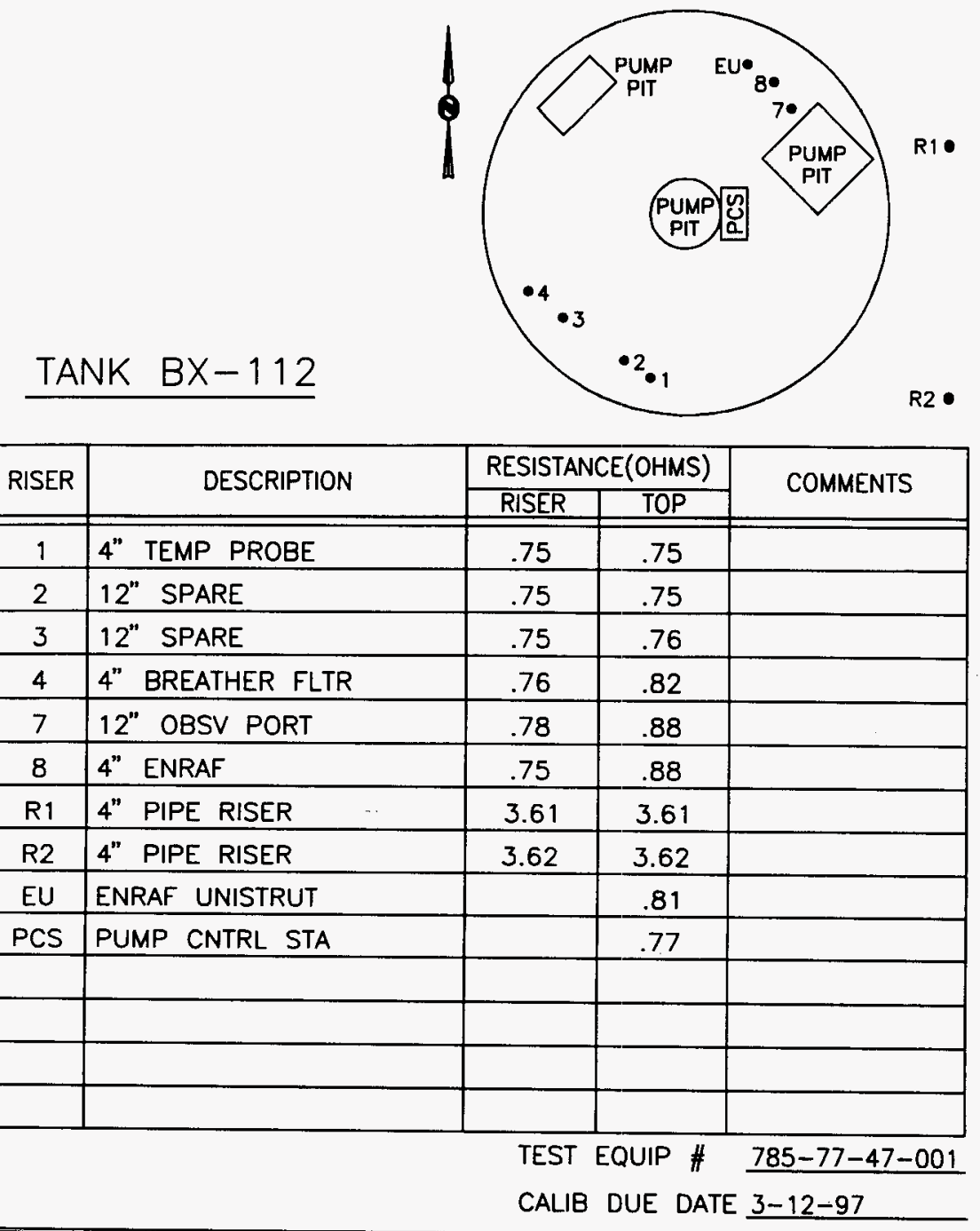




\section{RISER RESISTANCE DATA SHEET}

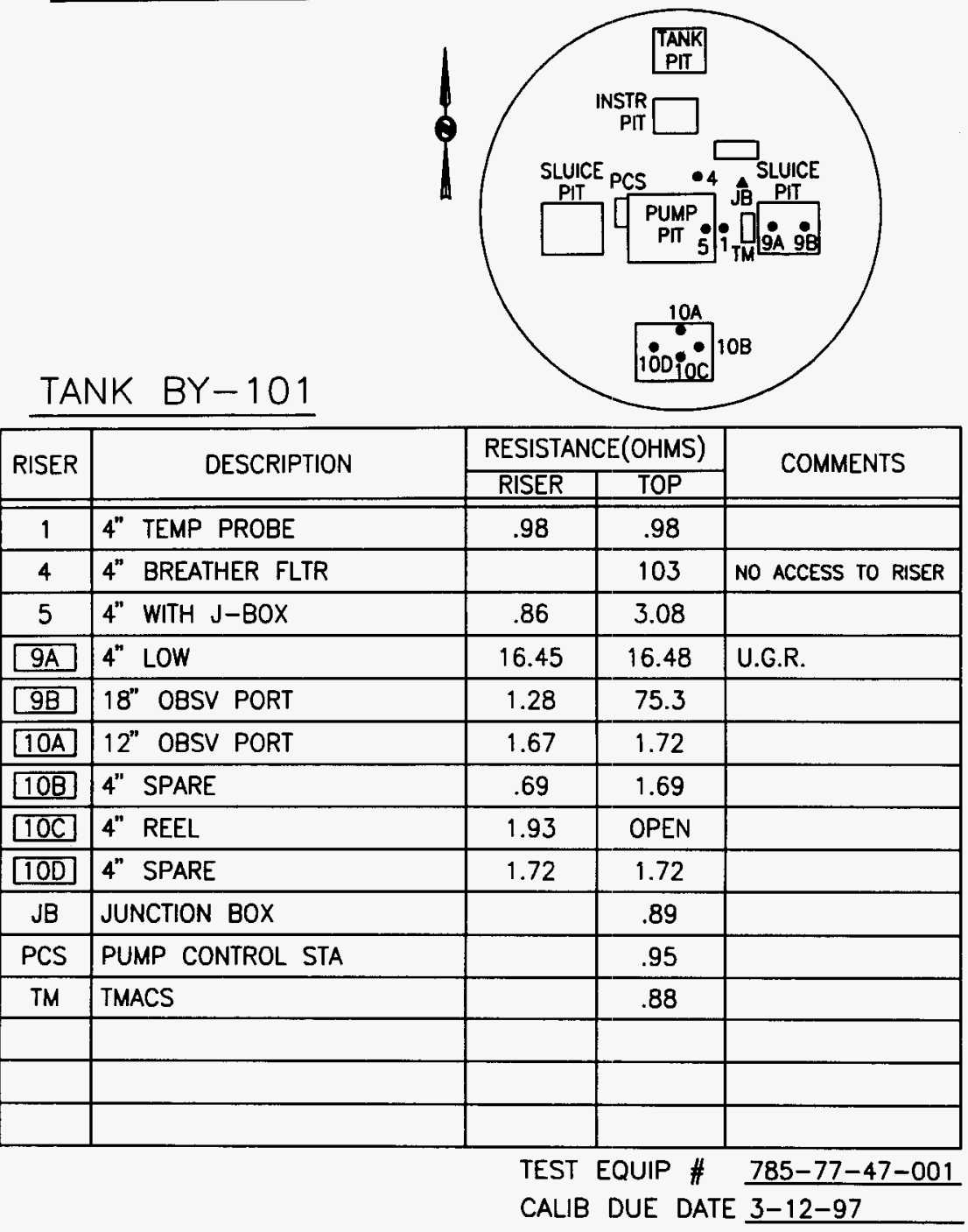




\section{RISER RESISTANCE DATA SHEET}

TANK BY-102
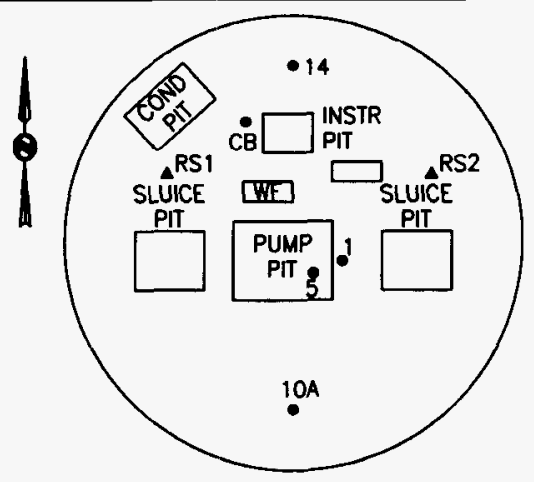

\begin{tabular}{|c|c|c|c|c|}
\hline \multirow{2}{*}{ RISER } & \multirow{2}{*}{ DESCRIPTION } & \multicolumn{2}{|c|}{ RESISTANCE(OHMS) } & \multirow{2}{*}{ COMMENTS } \\
\hline & & RISER & TOP & \\
\hline 1 & 4" LOW & 14.5 & & U.G.R. \\
\hline 5 & 4" REEL & .84 & OPEN & \\
\hline $10 A$ & 12" SHMS CONN & .85 & .88 & \\
\hline (14) & 4" BREATHER FLTR & 23.5 & 23.5 & U.G.R. \\
\hline $\mathrm{CB}$ & CONTROL BOX & & 1.25 & \\
\hline RS1 & RECEPTACLE STAND & & 1.08 & \\
\hline RS2 & RECEPTACLE STAND & & 28.3 & \\
\hline WF. & WF ENCLOSURE & & .94 & \\
\hline & & & & \\
\hline & & & & \\
\hline & & & & \\
\hline & & & & \\
\hline & & & & \\
\hline & & & & \\
\hline & & & & \\
\hline & & TES & QUIP \# & $785-77-47-001$ \\
\hline & & CALI & UE DA & $3-12-97$ \\
\hline
\end{tabular}


WHC-SD-WM-TR-034, REV. 0

\section{RISER RESISTANCE DATA SHEET}

TANK BY-103

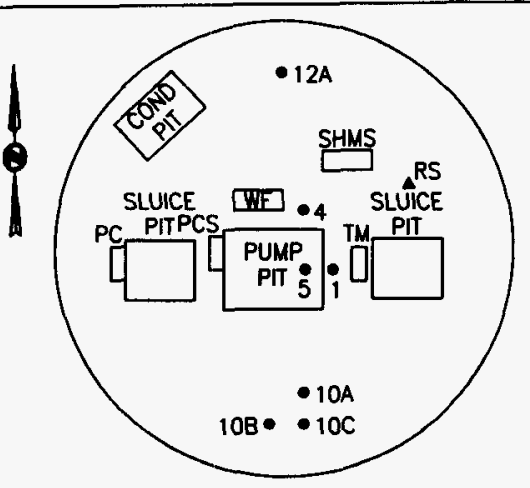

\begin{tabular}{|c|l|c|c|c|}
\hline \multirow{2}{*}{ RISER } & \multirow{2}{*}{ DESCRIPTION } & \multicolumn{2}{c|}{ RESISTANCE(OHMS) } & \multirow{2}{*}{ COMMENTS } \\
\cline { 3 - 4 } & & RISER & TOP & \\
\hline 1 & $4 "$ TEMP PROBE & .80 & .82 & \\
\hline 4 & $4 "$ TO SHMS & .81 & .91 & \\
\hline 5 & $4 "$ WITH J-BOX & .80 & .80 & \\
\hline $10 A$ & $4 "$ REEL & .81 & $.90(P)$ & PEDESTAL \\
\hline$[108$ & $12^{\prime \prime}$ OBSV PORT & .83 & .83 & \\
\hline$[10 C]$ & $4 "$ IN FIB LOW & .83 & & \\
\hline$[12 A]$ & $4 "$ BREATHER & .80 & .81 & \\
\hline PC & PUMP CONTROL & & 76.0 & \\
\hline PCS & PUMP CONTROL STA & & .81 & \\
\hline RS & RECEPTACLE STAND & & 195.1 & \\
\hline SC & SHMS & & .81 & \\
\hline TM & TMACS & & .80 & \\
\hline WF & WF ENCLOSURE & & .83 & \\
\hline & & & & \\
\hline & & & & \\
\hline
\end{tabular}

TEST EQUIP \# 785-77-47-001

CALIB DUE DATE 3-12-97 
WHC-SD-WM-TR-034, REV. 0

\section{RISER RESISTANCE DATA SHEET}

TANK BY -104

\begin{tabular}{|c|c|c|c|c|}
\hline \multirow{2}{*}{ RISER } & \multirow{2}{*}{ DESCRIPTION } & \multicolumn{2}{|c|}{ RESISTANCE(OHMS) } & \multirow{2}{*}{ COMMENTS } \\
\hline & & RISER & TOP & \\
\hline 1 & 4" TEMP PROBE & .84 & .88 & \\
\hline 4 & 4" BREATHER FLTR & .83 & .95 & \\
\hline 5 & 4" SPARE & .83 & .84 & \\
\hline 100 & 4" SHMS SETUP & .85 & .86 & \\
\hline $10 \mathrm{~A}$ & 18" OBSV PORT & .84 & .85 & \\
\hline $10 \mathrm{C}$ & 4" FIB LOW & .84 & & \\
\hline $10 \mathrm{~B}$ & 3" AIR CIRC & 325 & 325 & U.G.R. \\
\hline 11 & 8" SPARE & .84 & .84 & \\
\hline PCS & PUMP CONTROL STA & & .95 & \\
\hline TM & TMACS & & .85 & \\
\hline TPS & TEMP PROBE STAND & & .84 & \\
\hline & & & & \\
\hline & & & & \\
\hline & & & & \\
\hline & & & & \\
\hline
\end{tabular}

TEST EQUIP \# 785-77-47-001 CALIB DUE DATE 3-12-97 


\section{RISER RESISTANCE DATA SHEET}

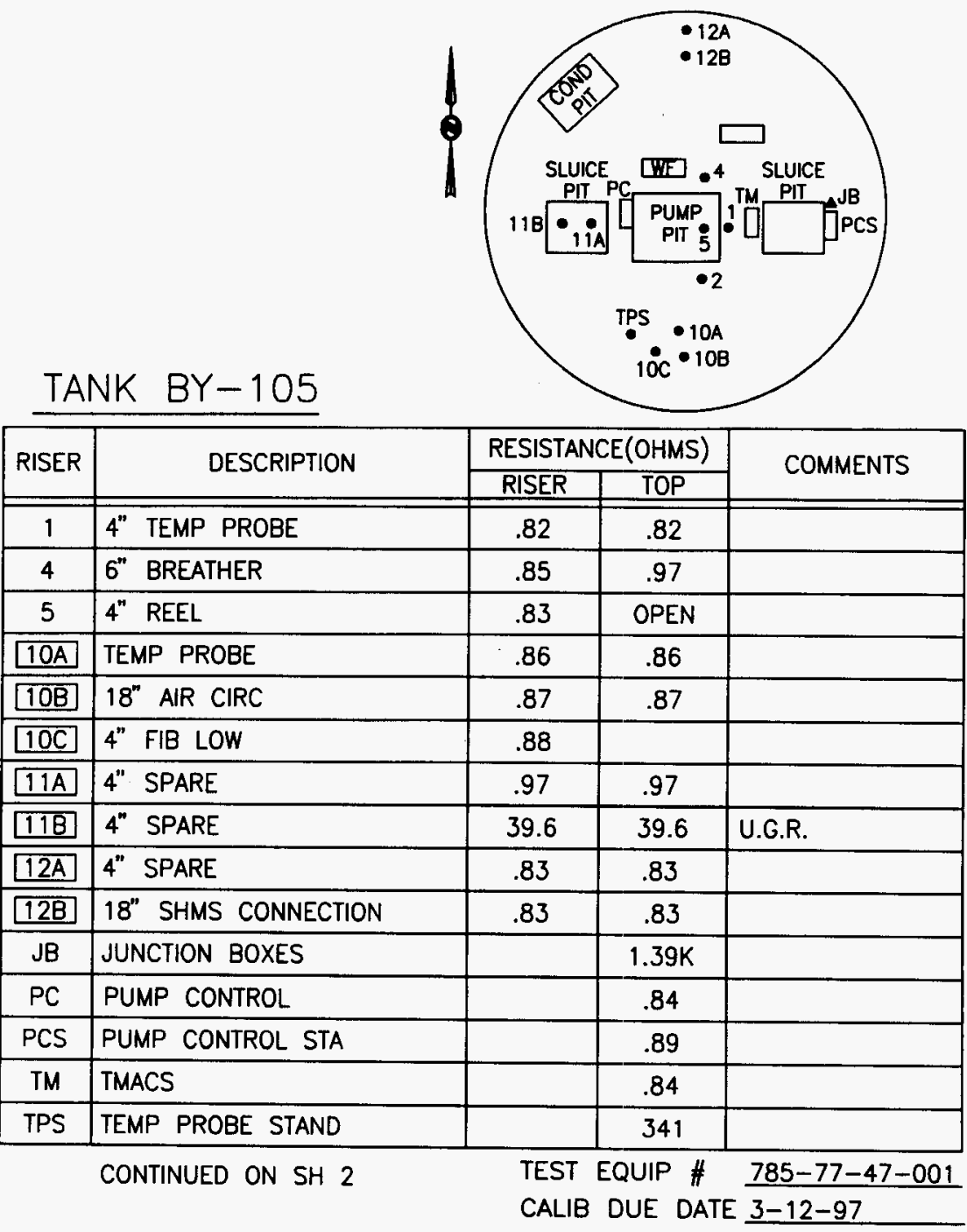




\section{RISER RESISTANCE DATA SHEET}

TANK BY-106

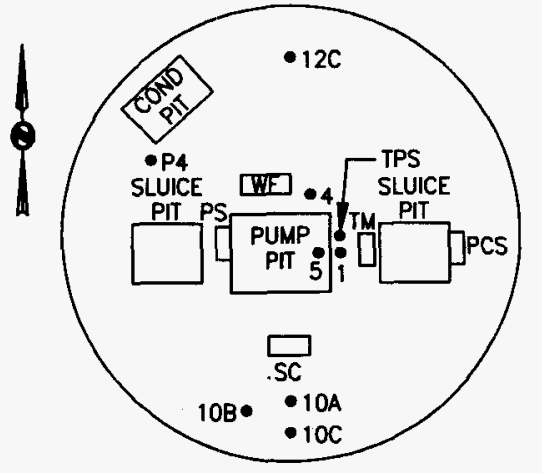

\begin{tabular}{|c|l|c|c|c|}
\hline \multirow{2}{*}{ RISER } & \multicolumn{1}{|c|}{ DESCRIPTION } & \multicolumn{2}{c|}{ RESISTANCE(OHMS) } & \multirow{2}{*}{ COMMENTS } \\
\cline { 3 - 4 } & & RISER & TOP & \\
\hline \hline 1 & $6 "$ TEMP PROBE & .84 & .88 & \\
\hline 4 & $4 "$ BREATHER & .84 & .87 & \\
\hline 5 & $4 "$ REEL & .83 & OPEN & \\
\hline $10 A$ & $18 "$ AIR CIRC & .84 & .87 & \\
\hline $10 \mathrm{~B}$ & $4 "$ SHMS CONNECTION & .84 & .86 & \\
\hline $10 \mathrm{C}$ & $4 "$ FIB LOW & .84 & & \\
\hline $12 \mathrm{C}$ & $4 "$ SPARE & .83 & 1.38 & \\
\hline P4 & $42 "$ EXHAUST DUCT & & 780 & \\
\hline PCS & PUMP CONTROL STA & & 1.04 & \\
\hline PS & PUMP STA & & .81 & \\
\hline SC & SHMS & & .82 & \\
\hline TM & TMACS & & .82 & \\
\hline TPS & TEMP PROBE STAND & & .83 & \\
\hline WF & WF ENCLOSURE & & .83 & \\
\hline & & & & \\
\hline
\end{tabular}

TEST EQUIP \# 785-77-47-001

CALIB DUE DATE 3-12-97 


\section{RISER RESISTANCE DATA SHEET}

\begin{tabular}{|c|c|c|c|c|}
\hline \multirow{2}{*}{ RISER } & \multirow{2}{*}{ DESCRIPTION } & \multicolumn{2}{|c|}{ RESISTANCE(OHMS) } & \multirow{2}{*}{ COMMENTS } \\
\hline & & RISER & TOP & \\
\hline 1 & 4" TEMP PROBE & .84 & .84 & \\
\hline 3 & 4" BREATHER FLTR & $*$ & 16.45 & *NO ACCESS \\
\hline 4 & 4" REEL & .84 & OPEN & \\
\hline 5 & 4" WITH J-BOX & .84 & .85 & \\
\hline 7 & $12^{\prime \prime}$ LOW & .82 & & \\
\hline 8 & $12^{\prime \prime}$ OBSV PORT & .84 & 82.3 & \\
\hline $9 \mathrm{~B}$ & $18^{n}$ SPARE & .98 & OPEN & \\
\hline $10 \mathrm{~B}$ & 12" DIP TUBES & .84 & .94 & \\
\hline 11 & $12^{n}$ SPARE & 1.72 & 1.75 & \\
\hline $12 \mathrm{~A}$ & 12" AIR CIRC & .84 & .84 & \\
\hline JB & JUNCTION BOX & & 16.12 & \\
\hline P1 & $3 n$ PIPE & & .88 & \\
\hline $\mathrm{P} 2$ & 3" PIPE & & .84 & \\
\hline $\mathrm{PC}$ & PUMP CONTROL & & 7.29 & \\
\hline LD & LEAK DETECTOR & & 7.29 & \\
\hline$T M$ & TMACS & & .84 & \\
\hline & & $\begin{array}{l}\text { TES } \\
\text { CALI }\end{array}$ & $\begin{array}{l}\text { QQUIP \# } \\
\text { DUE DAT }\end{array}$ & $=\frac{785-77-47-001}{3-12-97}$ \\
\hline
\end{tabular}




\section{RISER RESISTANCE DATA SHEET}

TANK BY-108
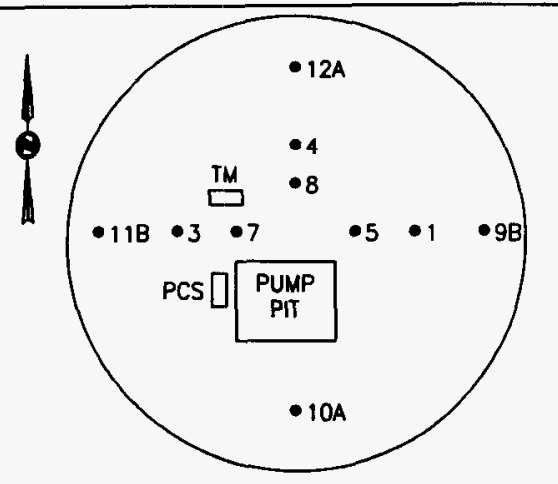

\begin{tabular}{|c|c|c|c|c|}
\hline \multirow{2}{*}{ RISER } & \multirow{2}{*}{ DESCRIPTION } & \multicolumn{2}{|c|}{ RESISTANCE(OHMS) } & \multirow{2}{*}{ COMMENTS } \\
\hline & & RISER & TOP & \\
\hline 1 & 4" BREATHER & .85 & .86 & \\
\hline 3 & 4" WITH J-BOX & .87 & .87 & \\
\hline 4 & 4" REEL & .86 & OPEN & \\
\hline 5 & 4" SPARE & .85 & .91 & \\
\hline 7 & 12" SPARE & .84 & .85 & \\
\hline 8 & 12" TЕMP BOX & .84 & .91 & \\
\hline $9 \mathrm{~B}$ & 12" SPARE & .87 & .97 & \\
\hline $10 \mathrm{~A}$ & 12" SPARE & .85 & .86 & \\
\hline $11 \mathrm{~B}$ & 4" SPARE & 1.07 & 1.97 & \\
\hline $12 A$ & 12" SHMS CONN & .84 & .87 & \\
\hline PCS & PUMP CONTROL STA & & .87 & \\
\hline TM & TMACS & & .85 & \\
\hline & & & & \\
\hline & & & & \\
\hline & & & & \\
\hline
\end{tabular}

TEST EQUIP \# 785-77-47-001

CALIB DUE DATE 3-12-97 


\section{RISER RESISTANCE DATA SHEET}

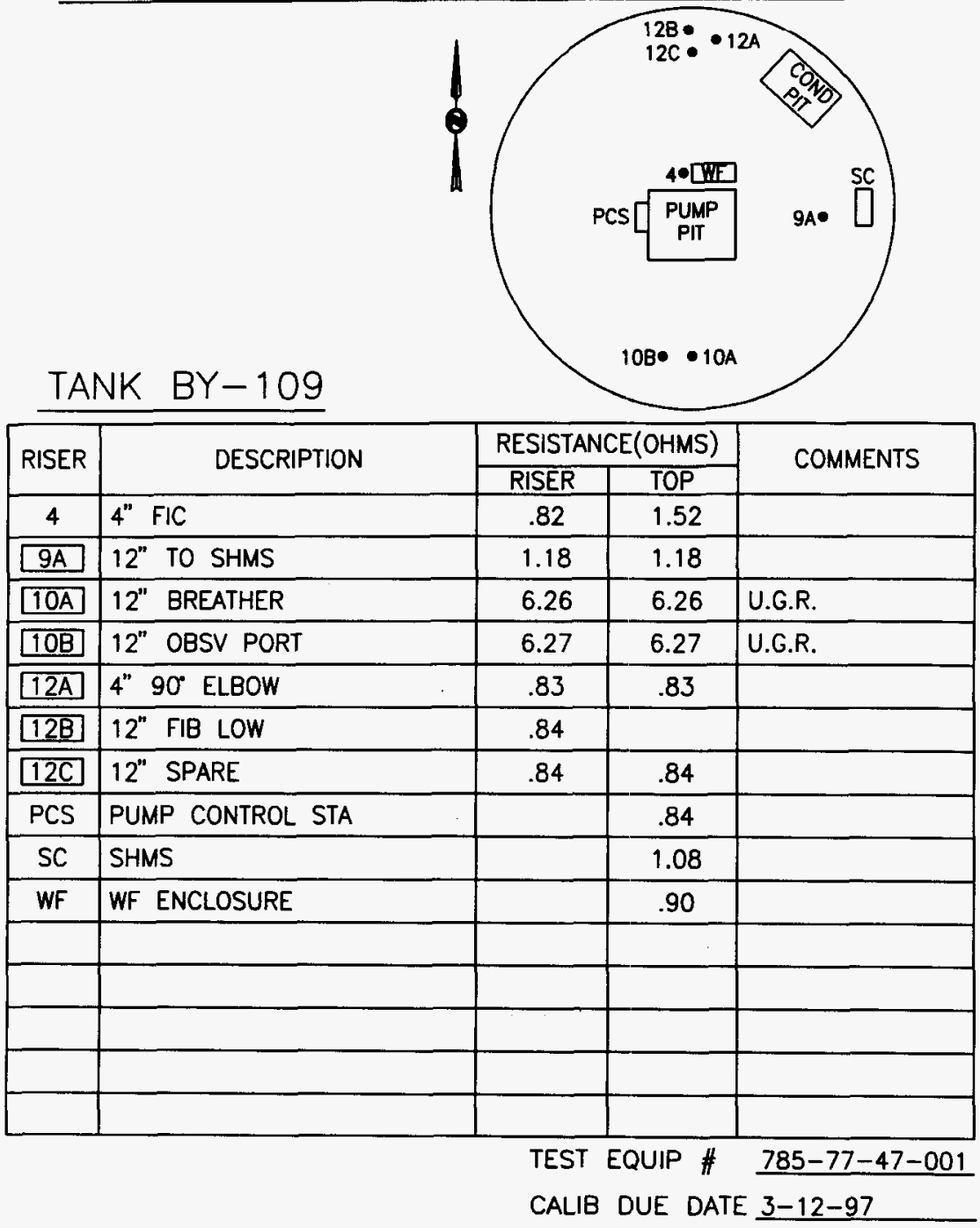




\section{RISER RESISTANCE DATA SHEET}

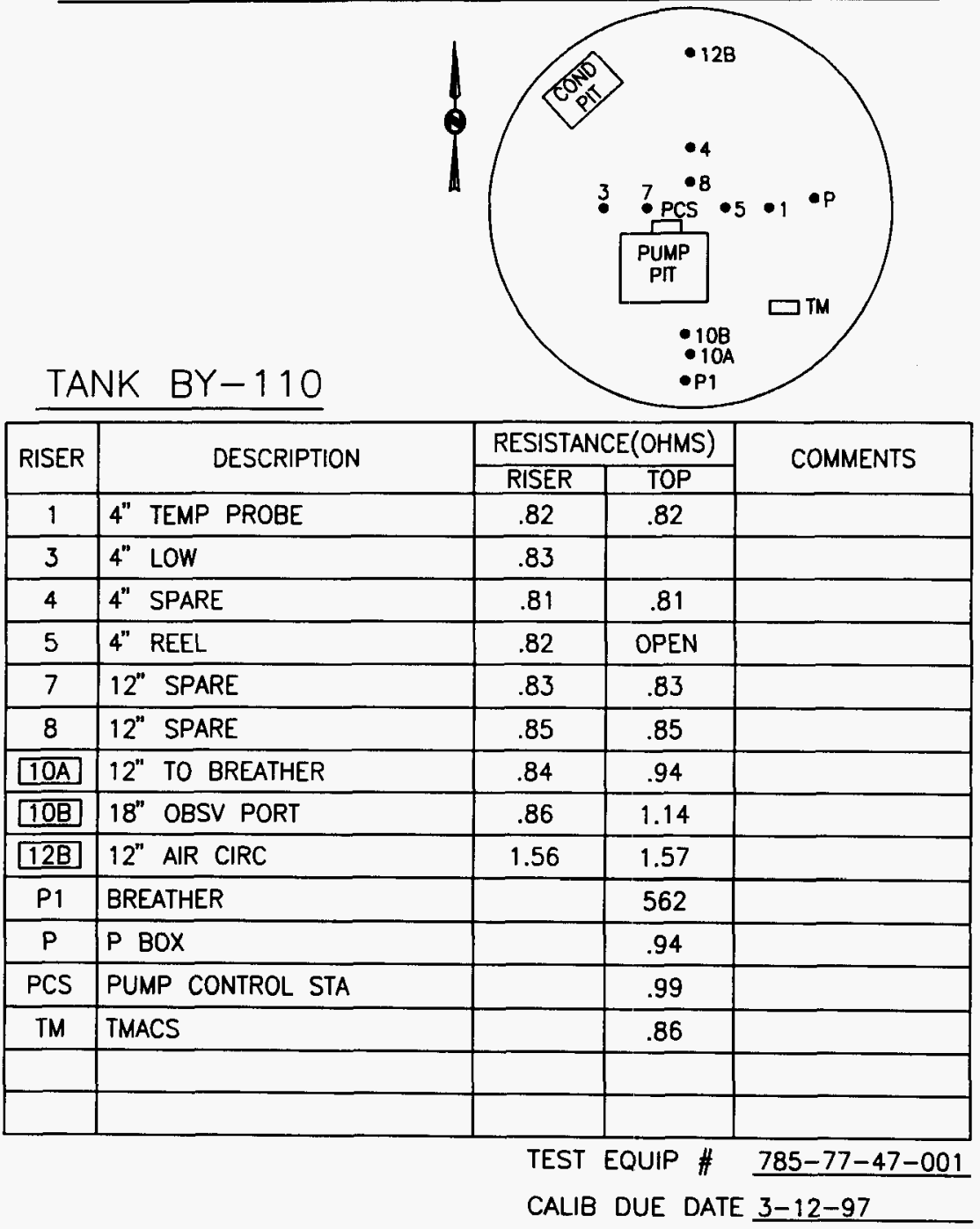




\section{RISER RESISTANCE DATA SHEET}

TANK BY-111

\begin{tabular}{|c|c|c|c|c|}
\hline \multirow{2}{*}{ RISER } & \multirow{2}{*}{ DESCRIPTION } & \multicolumn{2}{|c|}{ RESISTANCE(OHMS) } & \multirow{2}{*}{ COMMENTS } \\
\hline & & RISER & TOP & \\
\hline 1 & 4" LOW & 37.1 & & U.G.R. \\
\hline 4 & 4" BREATHER FLTR & 239 & 240 & U.G.R. \\
\hline 5 & 4" SPARE & .89 & .89 & \\
\hline $10 \mathrm{~B}$ & $12^{\prime \prime}$ AIR CIRC & 1.14 & 2.03 & \\
\hline $12 \mathrm{~A}$ & 12" OBSV PORT & 56.2 & 56.2 & U.G.R. \\
\hline (14) & 6" TEMP PROBE & 3.42 & 1.11 & G.T.W. \\
\hline (15) & 6" REEL & 45.1 & OPEN & U.G.R. \\
\hline PCS & PUMP CONTROL STA & & .85 & \\
\hline TM & TMACS & & .90 & \\
\hline & & & & \\
\hline & & & & \\
\hline & & & & \\
\hline & & & & \\
\hline & & & & \\
\hline & & & & \\
\hline & & $\begin{array}{l}\text { TEST } \\
\text { CALI }\end{array}$ & $\begin{array}{l}\text { QUIP \# } \\
\text { UE DA }\end{array}$ & $\begin{array}{l}785-77-47-001 \\
3-12-97\end{array}$ \\
\hline
\end{tabular}




\section{RISER RESISTANCE DATA SHEET}

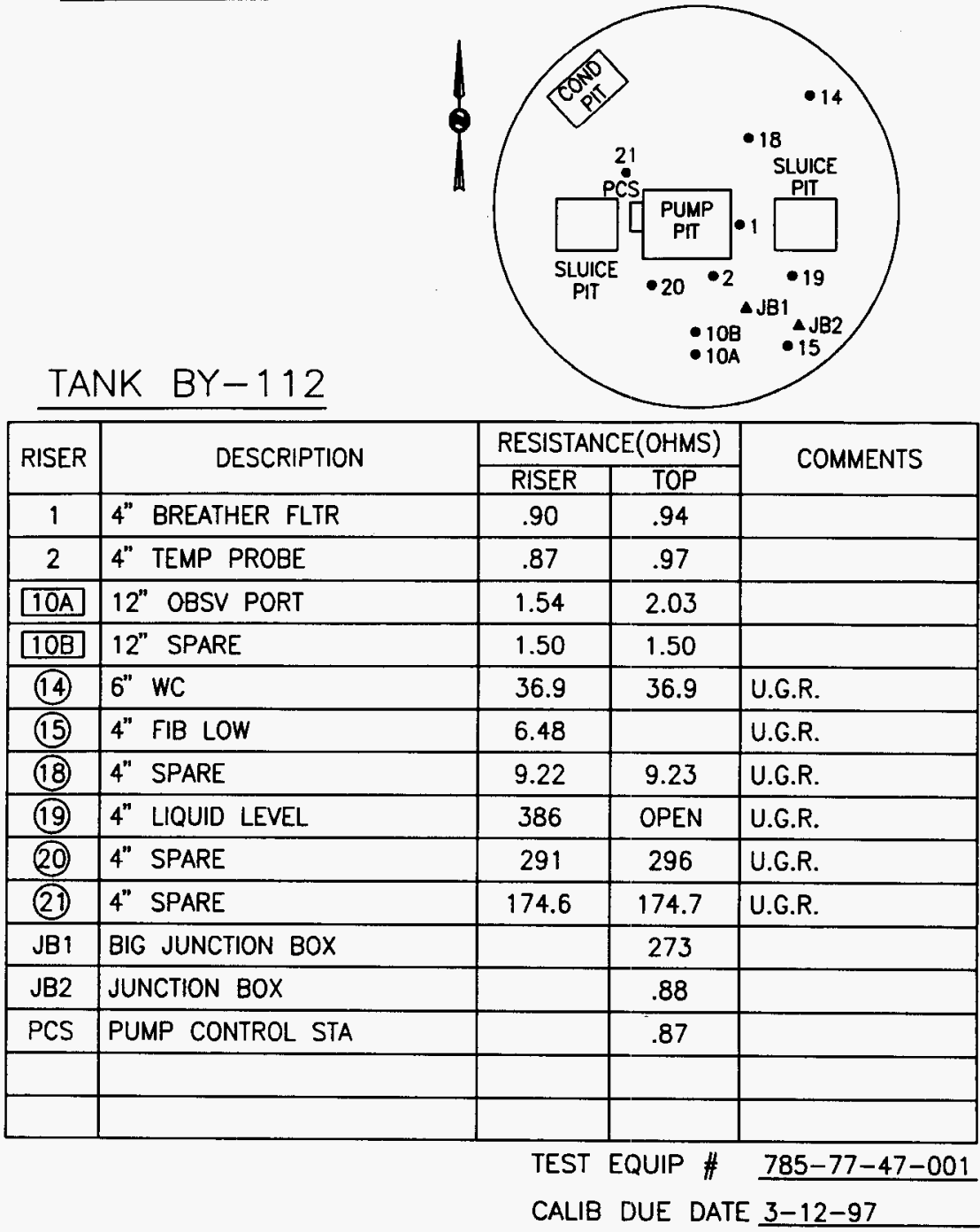


WHC-SD-WM-TR-034, REV. 0

\section{RISER RESISTANCE DATA SHEET}

TANK $\mathrm{C}-101$
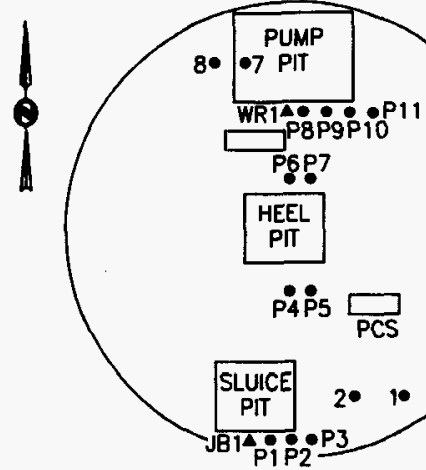

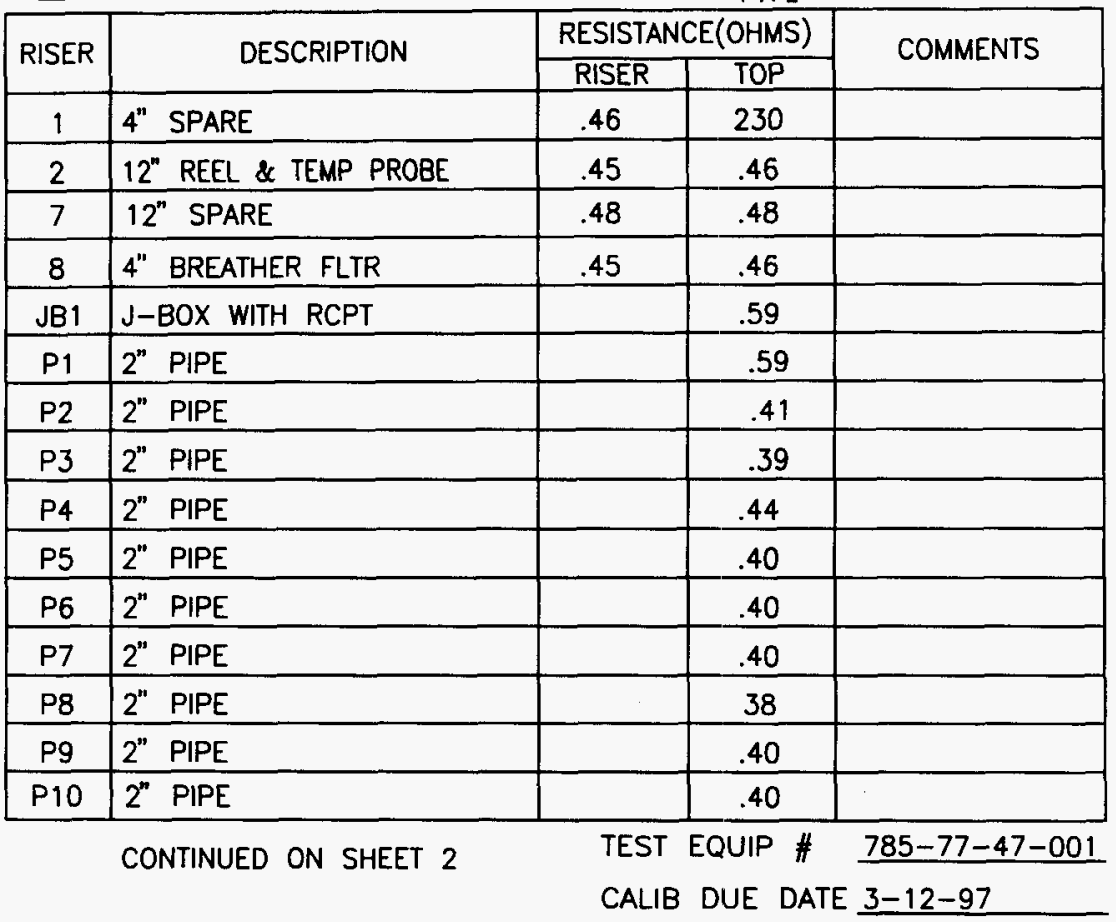




\section{RISER RESISTANCE DATA SHEET}

\section{TANK $\mathrm{C}-101$ CONTINUED FROM SHEET 1}

\begin{tabular}{|c|c|c|c|c|}
\hline \multirow{2}{*}{ RISER } & \multirow{2}{*}{ DESCRIPTION } & \multicolumn{2}{|c|}{ RESISTANCE(OHMS) } & \multirow{2}{*}{ COMMENTS } \\
\hline & & RISER & TOP & \\
\hline P11 & $2^{n}$ PIPE & & .40 & \\
\hline PCS & PUMP CONTROL STA & & .77 & \\
\hline WR1 & WELDING RECEPTACLE & & .64 & \\
\hline & & & & \\
\hline & & & & \\
\hline & & & & \\
\hline & & & & \\
\hline & & & & \\
\hline & & & & \\
\hline & & & & \\
\hline & & & & \\
\hline & & & & \\
\hline & & & & \\
\hline & & & & \\
\hline & & & & \\
\hline & & & & \\
\hline & & & & \\
\hline & & & & \\
\hline & & & & \\
\hline & & & & \\
\hline & & & & \\
\hline & & & & \\
\hline & & & & \\
\hline
\end{tabular}

TEST EQUIP \# 785-77-47-001 


\section{RISER RESISTANCE DATA SHEET}
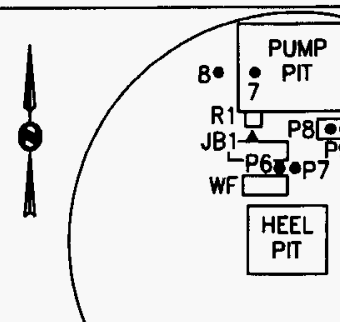

\section{P4ळP5}

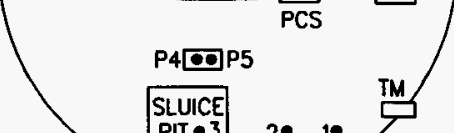

\section{TANK C-102

\begin{tabular}{|l|l}
\hline RISER & DESCRIPTION
\end{tabular}

SLUTCE

\begin{tabular}{|l|l|c|c|c|}
\hline 1 & $4 "$ & & & \\
\cline { 3 - 5 } & SPARE & .07 & .17 & \\
\hline 2 & $12^{\prime \prime}$ FIC & .07 & .09 & \\
\end{tabular}

\begin{tabular}{|l|l|}
\hline 3 & BREATHER \\
\hline 7 & $12^{\prime \prime}$ TEMP PROBE \\
\hline 8 & $4^{\prime \prime}$ SPARE \\
\hline
\end{tabular}

JB1

\begin{tabular}{|l|l|l|l|l|}
\hline P1 & 2" PIPE & & .08 & \\
\hline P2 & 2" PIPE & & .08 & \\
\hline P3 & 2" PIPE & & 81.0 & \\
\hline P4 & 2" PIPE & & .08 & \\
\hline P5 & 2" PIPE & & .13 & \\
\hline P6 & 2" PIPE & & .07 & \\
\hline P7 & 2" PIPE & & .07 & \\
\hline P8 & 2" PIPE & & .08 & \\
\hline P9 & 2" PIPE & & .08 & \\
\hline
\end{tabular}

CONTINUED ON SHEET 2

TEST EQUIP \# 785-77-47-001

CALIB DUE DATE 3-12-97 


\section{RISER RESISTANCE DATA SHEET}

TANK $\mathrm{C}-102$ CONTINUED FROM SHEET 1

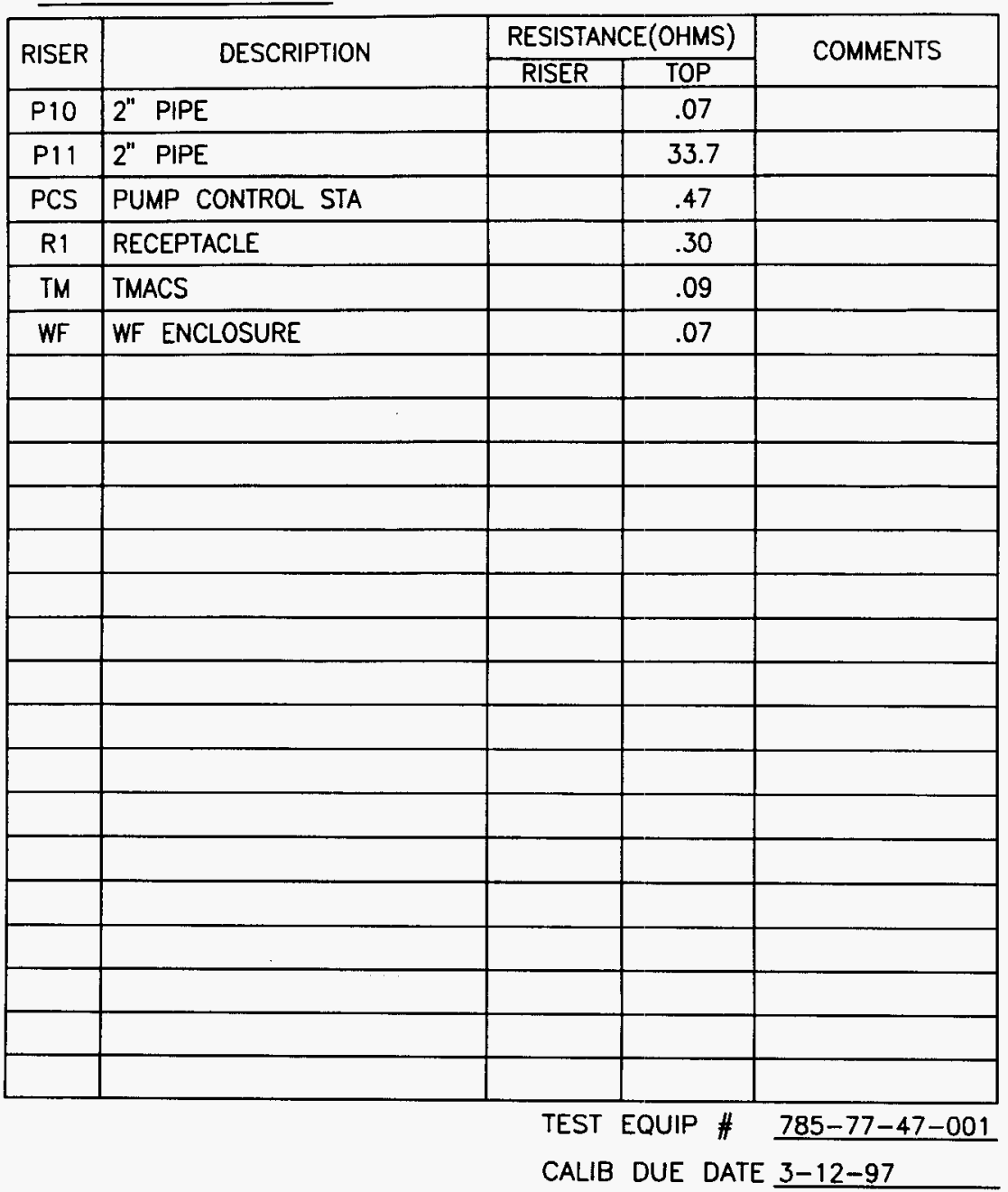


WHC-SD-WM-TR-034, REV. 0

\section{RISER RESISTANCE DATA SHEET}

TANK $\mathrm{C}-103$

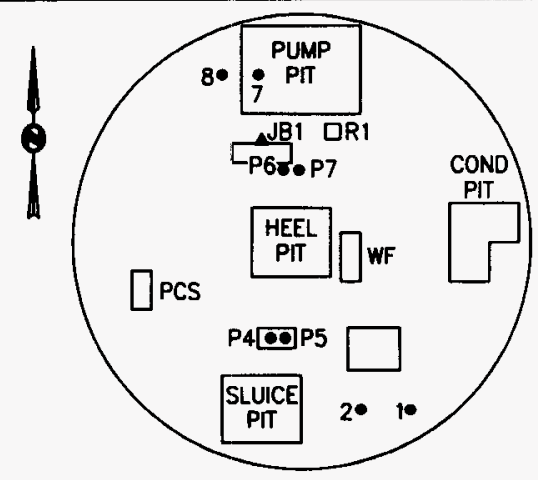

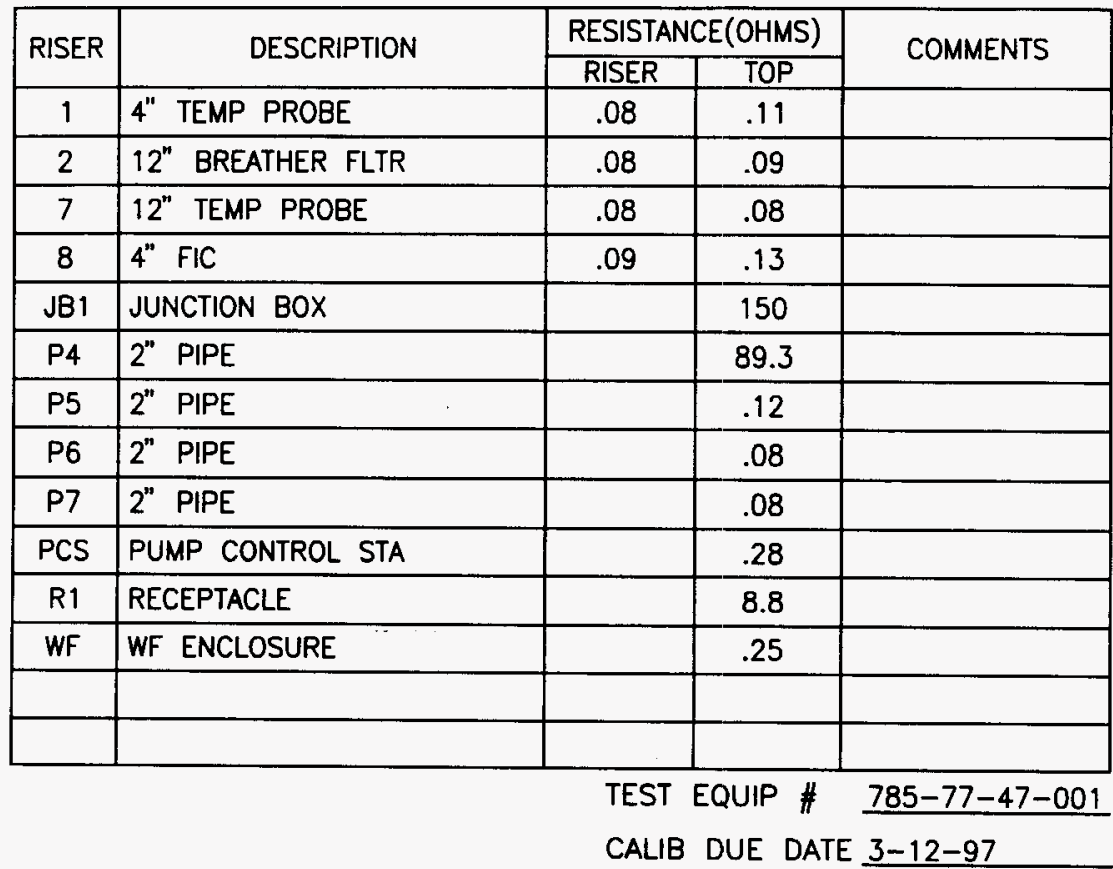




\section{RISER RESISTANCE DATA SHEET}

TANK $\mathrm{C}-104$

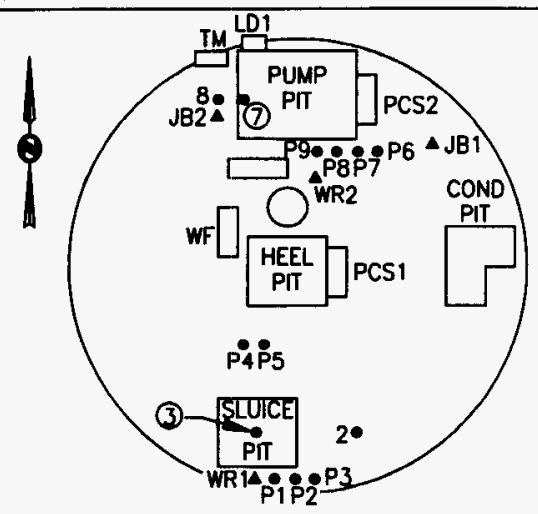

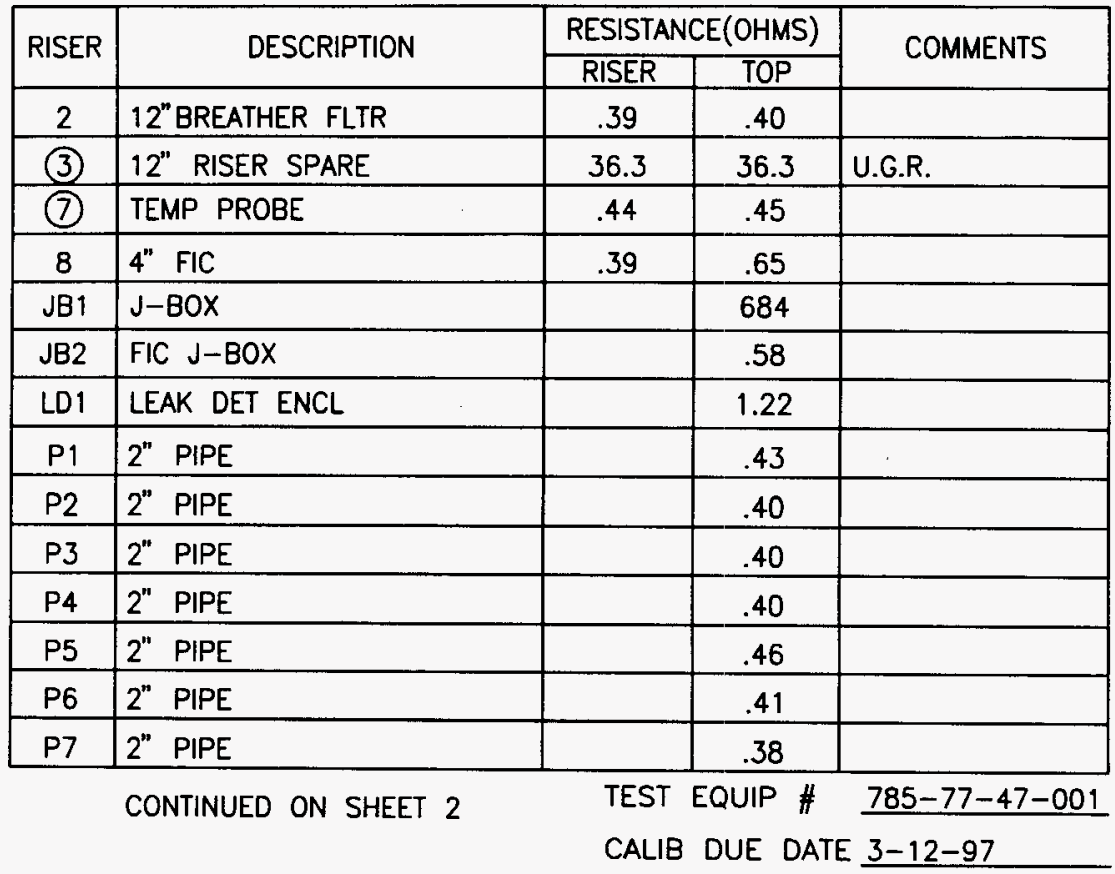


WHC-SD-WM-TR-034, REV. 0

\section{RISER RESISTANCE DATA SHEET}

TANK $\mathrm{C}-104$ CONTINUED FROM SHEET 1

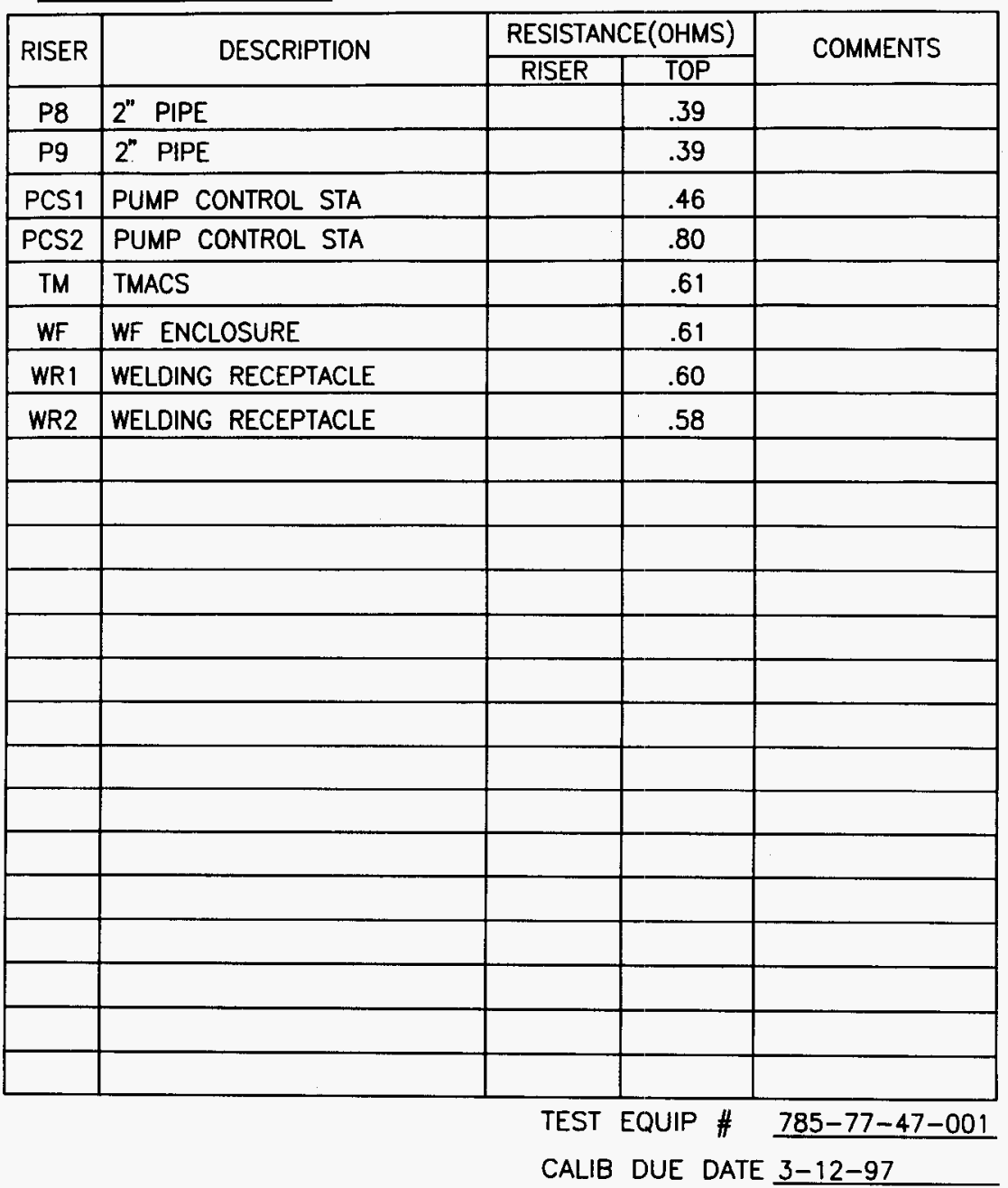




\section{RISER RESISTANCE DATA SHEET}

TANK C-105

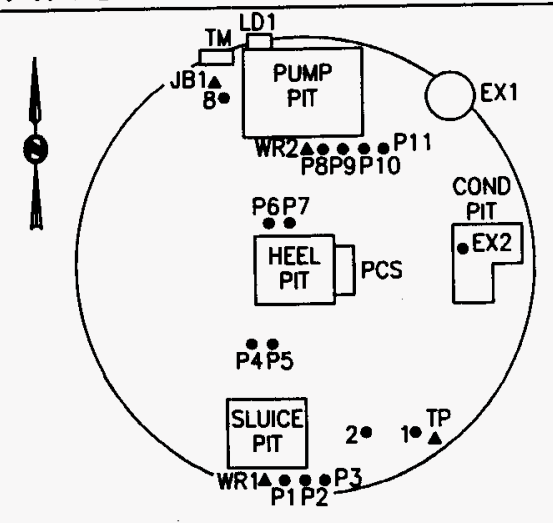

\begin{tabular}{|c|c|c|c|c|}
\hline \multirow{2}{*}{ RISER } & \multirow{2}{*}{ DESCRIPTION } & \multicolumn{2}{|c|}{ RESISTANCE(OHMS) } & \multirow{2}{*}{ COMMENTS } \\
\hline & & RISER & TOP & \\
\hline 1 & 4" TEMP PROBE & .40 & .41 & \\
\hline 2 & 12" INLET FILTER & .42 & .42 & \\
\hline 8 & 4" FIC & .40 & .42 & \\
\hline EX1 & EXHAUST & & .43 & \\
\hline $\mathrm{E} \times 2$ & EXHAUST DUCT RISER & & .40 & \\
\hline JB1 & FIC J-BOX & & .58 & \\
\hline LD1 & LEAK DET ENCL & & 1.37 & \\
\hline $\mathrm{P1}$ & $2^{n}$ PIPE & & .41 & \\
\hline $\mathrm{P} 2$ & 2" PIPE & & .42 & \\
\hline P3 & 2" PIPE & & .42 & \\
\hline P4 & 2" PIPE & & .40 & \\
\hline P5 & 2" PIPE & & .40 & \\
\hline P6 & 2" PIPE & & .40 & \\
\hline P7 & $2^{\prime \prime}$ PIPE & & .39 & \\
\hline & CONTINUED ON SHEET 2 & $\begin{array}{l}\text { TEST } \\
\text { CALII }\end{array}$ & $\begin{array}{l}\text { UIP \# } \\
\text { UE DA }\end{array}$ & $\begin{array}{l}785-77-47-001 \\
3-12-97\end{array}$ \\
\hline
\end{tabular}




\section{RISER RESISTANCE DATA SHEET}

TANK $\mathrm{C}-105$ CONTINUED From SHEET 1

\begin{tabular}{|c|c|c|c|c|}
\hline \multirow{2}{*}{ RISER } & \multirow{2}{*}{ DESCRIPTION } & \multicolumn{2}{|c|}{ RESISTANCE(OHMS) } & \multirow{2}{*}{ COMMENTS } \\
\hline & & RISER & TOP & \\
\hline P8 & $2^{n}$ PIPE & & .39 & \\
\hline P9 & $2^{n}$ PIPE & & .39 & \\
\hline P10 & $2^{\prime \prime}$ PIPE & & .39 & \\
\hline P11 & 2" PIPE & & .39 & \\
\hline PCS & PUMP CONTROL STA & & .65 & \\
\hline TM & TMACS & & .63 & \\
\hline TP & TEMP PROBE BOX & & .66 & \\
\hline WR1 & WELDING RECEPTACLE & & .53 & \\
\hline WR2 & WELDING RECEPTACLE & & 143.2 & \\
\hline & & & & \\
\hline & & & & \\
\hline & & & & \\
\hline & & & & \\
\hline & & & & \\
\hline & & & & \\
\hline & & & & \\
\hline & & & & \\
\hline & & & & \\
\hline & & & & \\
\hline & & & & \\
\hline & & & & \\
\hline & & & & \\
\hline & & & & \\
\hline & & $\begin{array}{l}\text { TEST } \\
\text { CALI }\end{array}$ & $\begin{array}{l}\text { QUIP \# } \\
\text { DUE DA }\end{array}$ & $\begin{array}{l}785-77-47-001 \\
3-12-97\end{array}$ \\
\hline
\end{tabular}




\section{RISER RESISTANCE DATA SHEET}

TANK C-106
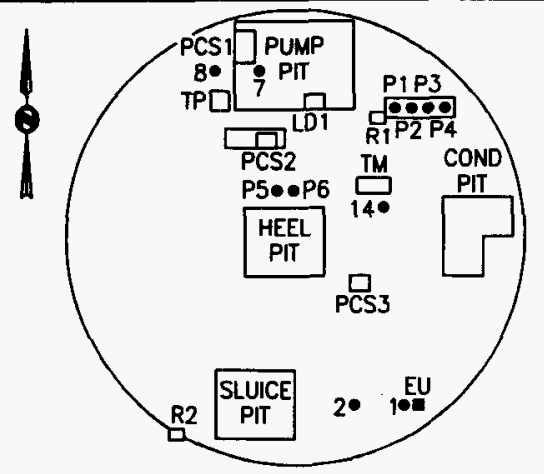

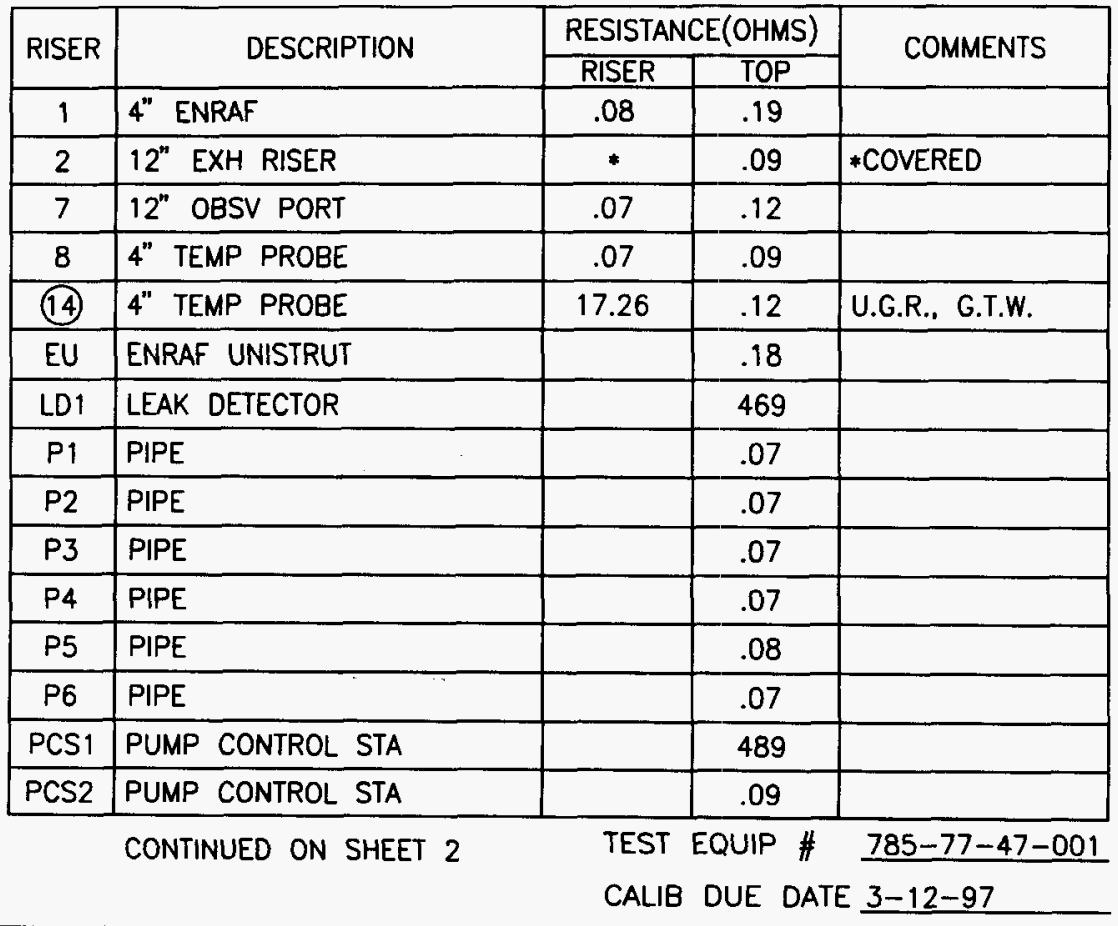




\section{RISER RESISTANCE DATA SHEET}

TANK C-106 CONTINUED FROM SHEET 1

\begin{tabular}{|c|c|c|c|c|}
\hline \multirow{2}{*}{ RISER } & \multirow{2}{*}{ DESCRIPTION } & \multicolumn{2}{|c|}{ RESISTANCE(OHMS) } & \multirow{2}{*}{ COMMENTS } \\
\hline & & RISER & TOP & \\
\hline PCS3 & PUMP CONTROL STA & & .24 & \\
\hline R1 & RECEPTACLE & & .10 & \\
\hline R2 & RECEPTACLE & & 156 & \\
\hline TM & TMACS & & .20 & \\
\hline TP & TEMP PROBE BOX & & 2.71 & \\
\hline & & & & \\
\hline & & & & \\
\hline & & & & \\
\hline & & & & \\
\hline & & & & \\
\hline & & & & \\
\hline & & & & \\
\hline & & & & \\
\hline & & & & \\
\hline & & & & \\
\hline & & & & \\
\hline & & & & \\
\hline & & & & \\
\hline & & & & \\
\hline & & & & \\
\hline & & & & \\
\hline & & & & \\
\hline & & & & \\
\hline
\end{tabular}

TEST EQUIP \# 785-77-47-001 CALIB DUE DATE $3-12-97$ 


\section{RISER RESISTANCE DATA SHEET}

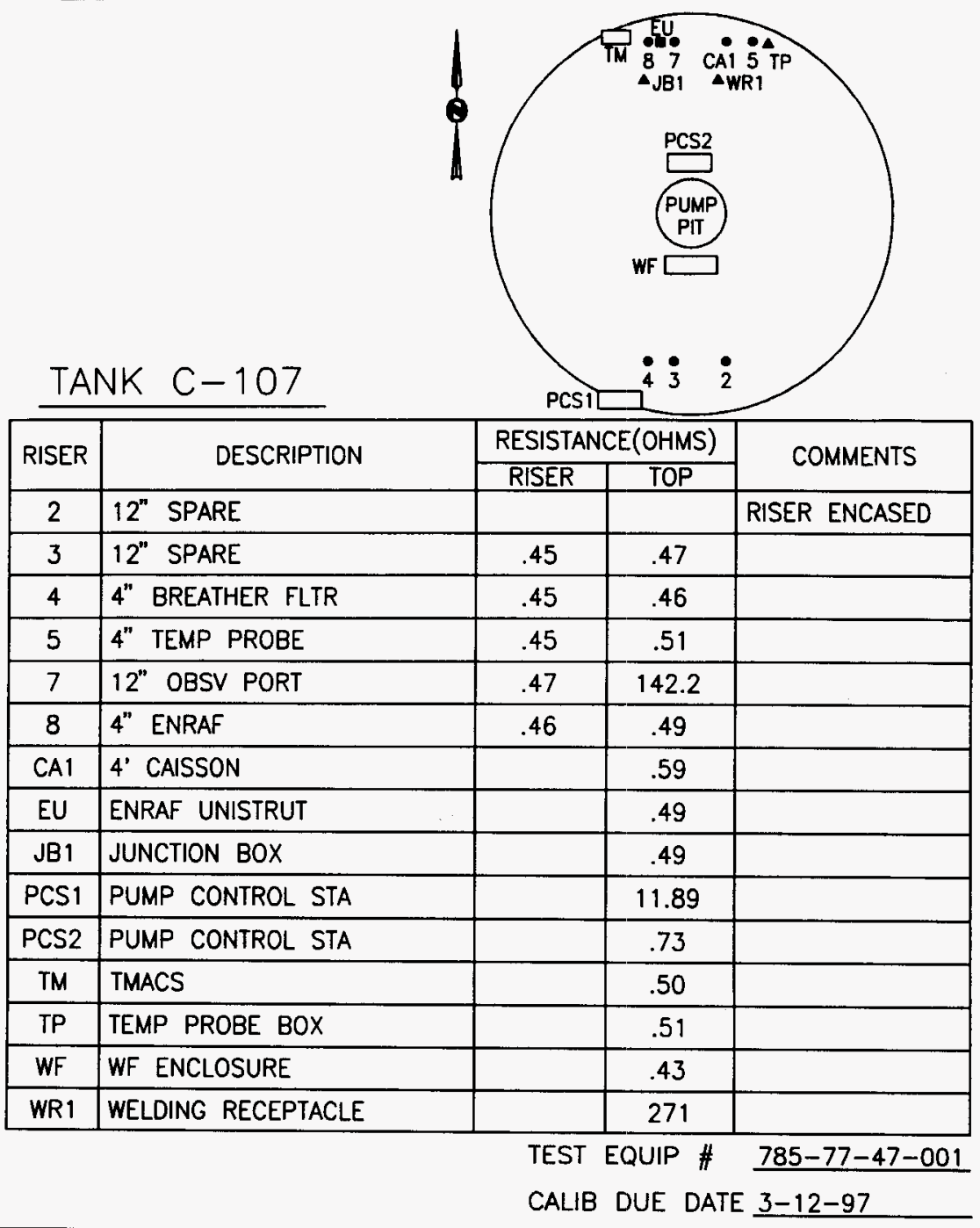


WHC-SD-WM-TR-034, REV. 0

\section{RISER RESISTANCE DATA SHEET}

TANK $\mathrm{C}-108$

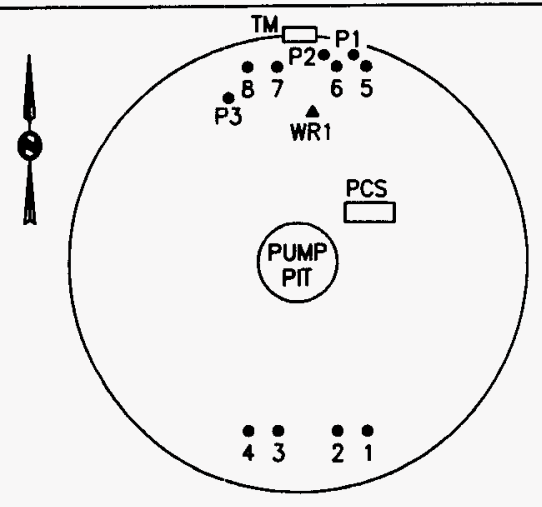

\begin{tabular}{|c|l|c|c|c|}
\hline \multirow{2}{*}{ RISER } & \multirow{2}{*}{ DESCRIPTION } & \multicolumn{2}{c|}{ RESISTANCE(OHMS) } & \multirow{2}{*}{ COMMENTS } \\
\cline { 3 - 4 } & & RISER & TOP & \\
\hline 1 & $4^{\prime \prime}$ THERMO TREE & .44 & .44 & \\
\hline 2 & $12^{n}$ DIP TUBES & .43 & .44 & \\
\hline 3 & $12^{n}$ SPARE & .44 & .44 & \\
\hline 4 & $4^{\prime \prime}$ BREATHER FLTR & .44 & .67 & \\
\hline 5 & $4^{n}$ TEMP PROBE & .45 & .59 & \\
\hline 6 & $12^{\prime \prime}$ SPARE & .52 & .54 & \\
\hline 7 & $12^{\prime \prime}$ OBSV PORT & .49 & .70 & \\
\hline 8 & $4^{n}$ REEL & .44 & $.50(P)$ & PEDESTAL \\
\hline P1 & $2^{\prime \prime}$ PIPE & & .43 & \\
\hline P2 & $2^{n}$ PIPE & & .43 & \\
\hline P3 & $1^{\prime \prime}$ CONDUIT & & .48 & \\
\hline PCS & PUMP CONTROL STA & & .75 & \\
\hline TM & TMACS & & .47 & \\
\hline WR1 & WELDING RECEPTACLE & & .83 & \\
\hline
\end{tabular}

TEST EQUIP \# 785-77-47-001

CALIB DUE DATE 3-12-97 
WHC-SD-WM-TR-034, REV. 0

\section{RISER RESISTANCE DATA SHEET}

TANK $\mathrm{C}-109$

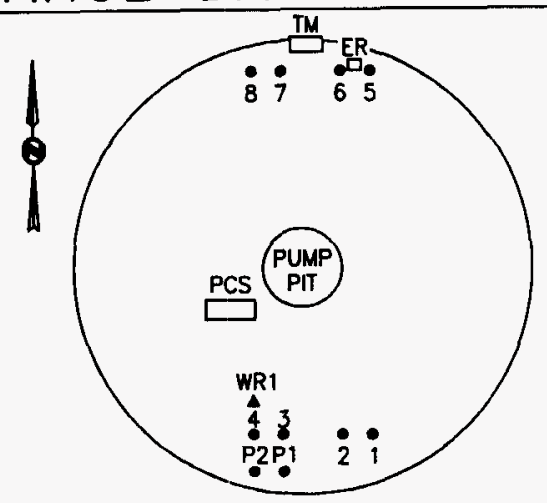

\begin{tabular}{|c|c|c|c|c|}
\hline \multirow{2}{*}{ RISER } & \multirow{2}{*}{ DESCRIPTION } & \multicolumn{2}{|c|}{ RESISTANCE(OHMS) } & \multirow{2}{*}{ COMMENTS } \\
\hline & & RISER & TOP & \\
\hline 1 & 4" REEL & .43 & $.49(P)$ & PEDESTAL \\
\hline 2 & $12^{\prime \prime}$ SPARE & .42 & .43 & \\
\hline 3 & $12^{n}$ TEMP PROBE & .43 & .43 & \\
\hline 4 & 4" BREATHER FLTR & .42 & .43 & \\
\hline 5 & $4^{n}$ SPARE & .43 & 6.47 & \\
\hline 6 & 12" SPARE & .41 & .41 & \\
\hline 7 & $12^{\prime \prime}$ OBSV PORT & .42 & .42 & \\
\hline 8 & 4" TEMP PROBE & .44 & .45 & \\
\hline$E R$ & ELEC RACK & & .45 & \\
\hline P1 & 2" PIPE & & .44 & \\
\hline $\mathrm{P} 2$ & 2" PIPE & & .45 & \\
\hline PCS & PUMP CONTROL STA & & .65 & \\
\hline TM & TMACS & & .45 & \\
\hline WR1 & WELDING RECEPTACLE & & .90 & \\
\hline
\end{tabular}

TEST EQUIP \# 785-77-47-001

CALIB DUE DATE 3-12-97 
WHC-SD-WM-TR-034, REV. 0

\section{RISER RESISTANCE DATA SHEET}

TANK $\mathrm{C}-110$

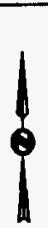

\begin{tabular}{|c|c|c|c|c|}
\hline \multirow{2}{*}{ RISER } & \multirow{2}{*}{ DESCRIPTION } & \multicolumn{2}{|c|}{ RESISTANCE(OHMS) } & \multirow{2}{*}{ COMMENTS } \\
\hline & & RISER & TOP & \\
\hline 1 & $4^{\prime \prime}$ SLUDGE MEAS & .42 & .43 & \\
\hline 2 & $12^{\prime \prime}$ SPARE & .42 & .43 & \\
\hline 3 & 12"BREATHER FLTR & .44 & .46 & \\
\hline 4 & 4" REEL & .43 & $.43(P)$ & PEDESTAL \\
\hline 5 & $4^{\prime \prime}$ SPARE & .43 & .43 & \\
\hline 6 & 12" SPARE & .42 & .52 & \\
\hline 7 & $12^{\prime \prime}$ OBSV PORT & .43 & .43 & \\
\hline 8 & $4^{\prime \prime}$ TEMP PROBE & .42 & .43 & \\
\hline P1 & $2^{\prime \prime}$ PIPE & & .42 & \\
\hline $\mathrm{P} 2$ & 2" PIPE & & .42 & \\
\hline P3 & 1" CONDUIT & & .59 & \\
\hline PCS & PUMP CONTROL STA & & .45 & \\
\hline & PUMP PIT & & $1.6 \mathrm{~K}$ & \\
\hline WF & WF ENCLOSURE & & .44 & \\
\hline WR1 & WELDING RECEPTACLE & & .80 & \\
\hline
\end{tabular}

TEST EQUIP \# 785-77-47-001

CALIB DUE DATE 3-12-97 


\section{RISER RESISTANCE DATA SHEET}

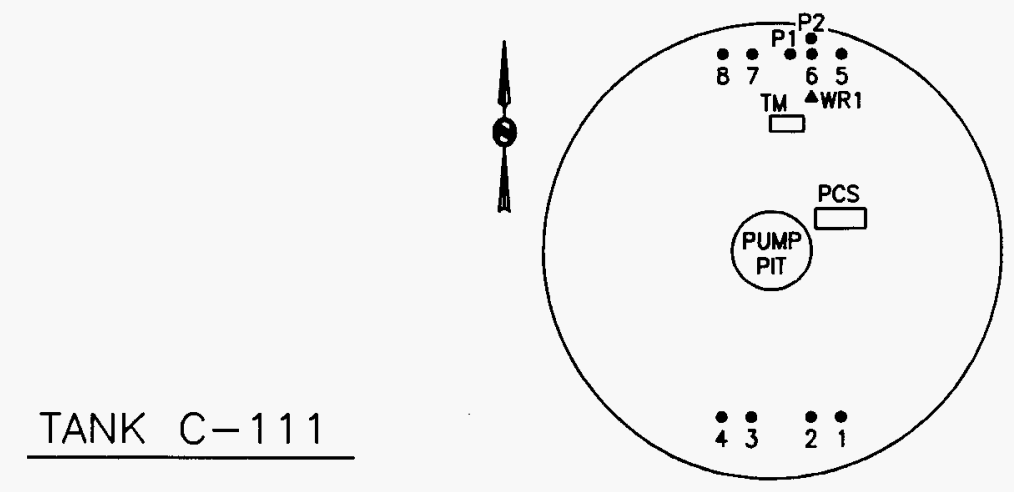

\begin{tabular}{|c|c|c|c|c|}
\hline \multirow{2}{*}{ RISER } & \multirow{2}{*}{ DESCRIPTION } & \multicolumn{2}{|c|}{ RESISTANCE(OHMS) } & \multirow{2}{*}{ COMMENTS } \\
\hline & & RISER & TOP & \\
\hline 1 & 4" SPARE & .46 & .54 & \\
\hline 2 & 12" SPARE & .44 & .44 & \\
\hline 3 & 12" BREATHER & .44 & .49 & \\
\hline 4 & $4 "$ SPARE & .46 & .56 & \\
\hline 5 & 4" TEMP PROBE & .44 & .48 & \\
\hline 6 & $12^{\prime \prime}$ OBSV PORT & .44 & .47 & \\
\hline 7 & 12" SPARE & .45 & .55 & \\
\hline 8 & 4" REEL & .44 & 138.8 & \\
\hline $\mathrm{P1}$ & 2" PIPE & & .45 & \\
\hline $\mathrm{P} 2$ & 2" PIPE & & .45 & \\
\hline PCS & PUMP CONTROL STA & & .73 & \\
\hline TM & TMACS & & .45 & \\
\hline WR1 & WELDING RECEPTACLE & & .86 & \\
\hline & & & & \\
\hline
\end{tabular}

TEST EQUIP \# 785-77-47-001 CALIB DUE DATE $\underline{3-12-97}$ 


\section{RISER RESISTANCE DATA SHEET}

TANK $\mathrm{C}-112$

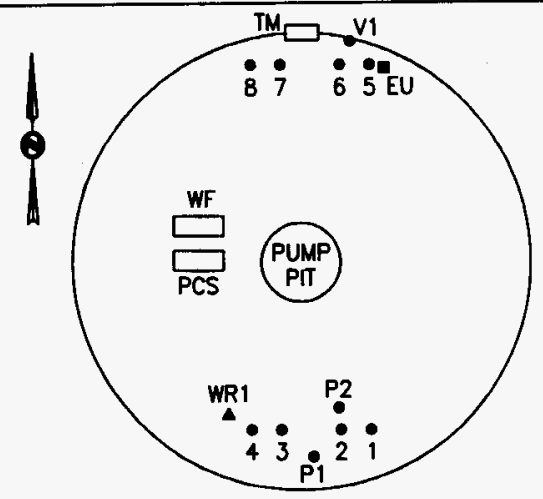

\begin{tabular}{|c|l|c|c|c|}
\hline \multirow{2}{*}{ RISER } & \multicolumn{1}{|c|}{ DESCRIPTION } & \multicolumn{2}{c|}{ RESISTANCE(OHMS) } & \multirow{2}{*}{ COMMENTS } \\
\cline { 3 - 4 } & \multicolumn{1}{|c|}{} & RISER & TOP & \\
\hline 1 & 4 " TEMP PROBE & .41 & .61 & \\
\hline 2 & $12^{\prime \prime}$ SPARE & .41 & .42 & \\
\hline 3 & $12^{\prime \prime}$ SPARE & 70 & 70 & U.G.R. \\
\hline 4 & $4 "$ BREATHER FLTR & .43 & .48 & \\
\hline 5 & $4 "$ ENRAF & .41 & .44 & \\
\hline 6 & $12 "$ SPARE & .41 & .42 & \\
\hline 7 & $12 "$ OBSV PORT & .41 & .44 & \\
\hline 8 & $4 "$ TEMP PROBE & .42 & .43 & \\
\hline EU & ENRAF UNISTRUT & & .44 & \\
\hline P1 & 2" PIPE & & .43 & \\
\hline P2 & 2" PIPE & & .41 & \\
\hline PCS & PUMP CONTROL STA & & 1.17 & \\
\hline & PUMP PIT & & 1.03 & \\
\hline TM & TMACS & & .43 & \\
\hline
\end{tabular}

CONTINUED ON SHEET 2

TEST EQUIP \# 785-77-47-001

CALIB DUE DATE 3-12-97 


\section{RISER RESISTANCE DATA SHEET}

TANK $\mathrm{C}-112$ CONTINUED FROM SHEET 1

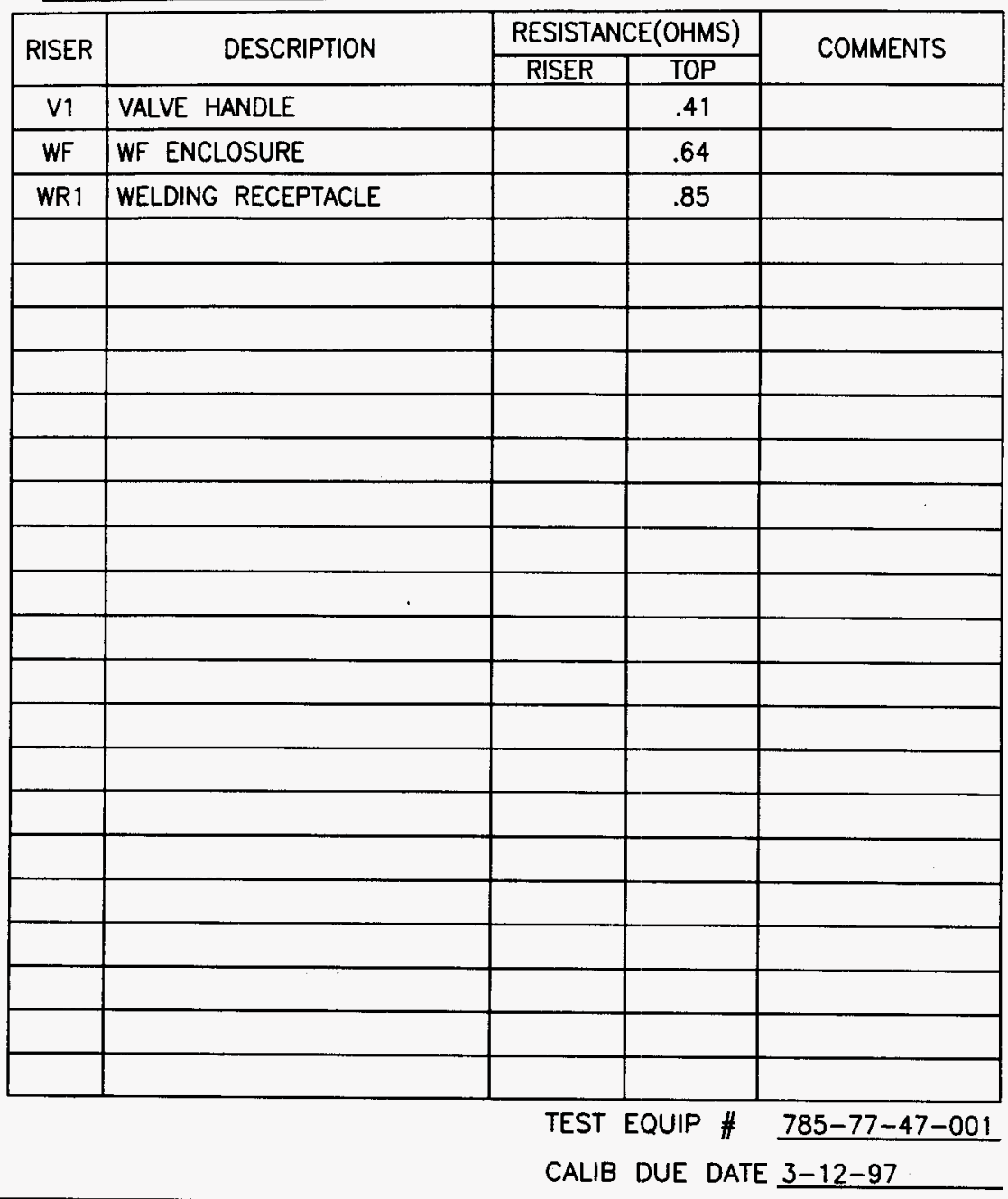




\section{RISER RESISTANCE DATA SHEET}

TANK $\quad \mathrm{C}-201$

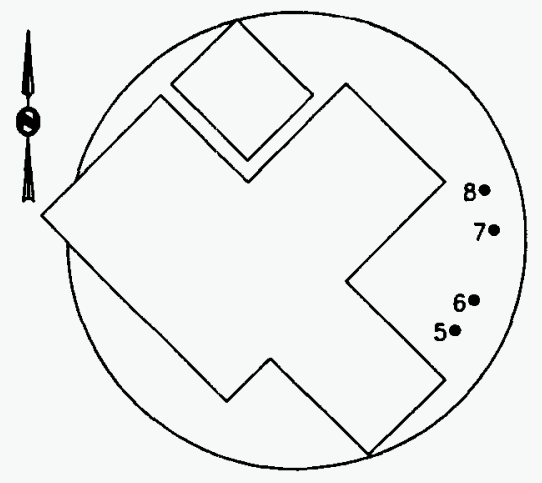

\begin{tabular}{|c|c|c|c|c|}
\hline \multirow{2}{*}{ RISER } & \multirow{2}{*}{ DESCRIPTION } & \multicolumn{2}{|c|}{ RESISTANCE(OHMS) } & \multirow{2}{*}{ COMMENTS } \\
\hline & & RISER & TOP & \\
\hline 5 & 4" REEL & .99 & & \\
\hline 6 & 12" TEMPERATURE & .51 & .53 & \\
\hline 7 & 12" OBSV PORT & 21.2 & 21.2 & U.G.R. \\
\hline 8 & 4" BREATHER FLTR & .53 & .67 & \\
\hline & & & & \\
\hline & & & & \\
\hline & & & & \\
\hline & & & & \\
\hline & & & & \\
\hline & & & & \\
\hline & & & & \\
\hline & & & & \\
\hline & & & & \\
\hline & & & & \\
\hline & & $\begin{array}{l}\text { TES } \\
\text { CAL }\end{array}$ & $\begin{array}{l}\text { UIP \# } \\
\text { UE DA }\end{array}$ & $\begin{array}{l}785-77-47-001 \\
3-12-97\end{array}$ \\
\hline
\end{tabular}




\section{RISER RESISTANCE DATA SHEET}

TANK C-202

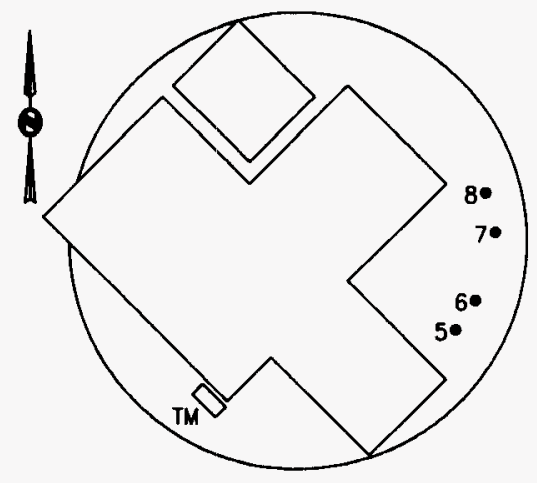

\begin{tabular}{|c|c|c|c|c|}
\hline \multirow{2}{*}{ RISER } & \multirow{2}{*}{ DESCRIPTION } & \multicolumn{2}{|c|}{ RESISTANCE(OHMS) } & \multirow{2}{*}{ COMMENTS } \\
\hline & & RISER & TOP & \\
\hline 5 & 4" REEL & 36.6 & & U.G.R. \\
\hline 6 & 12" TEMPERATURE & .52 & .52 & \\
\hline 7 & 12" OBSV PORT & .54 & 1.41 & \\
\hline 8 & $\begin{array}{lll}\text { 4" } & \text { BREATHER FLTR } \\
\end{array}$ & .58 & .59 & \\
\hline \multirow[t]{2}{*}{ TM } & TMACS & & .51 & \\
\hline & .. & & & \\
\hline & & & & \\
\hline & & & & \\
\hline & & & & \\
\hline & & & & \\
\hline & & & & \\
\hline & & & & \\
\hline & & & & \\
\hline & & TEST & UIP \# & $785-77-47-001$ \\
\hline & & CALI & JE DAT & $3-12-97$ \\
\hline
\end{tabular}




\section{RISER RESISTANCE DATA SHEET}

TANK C-203

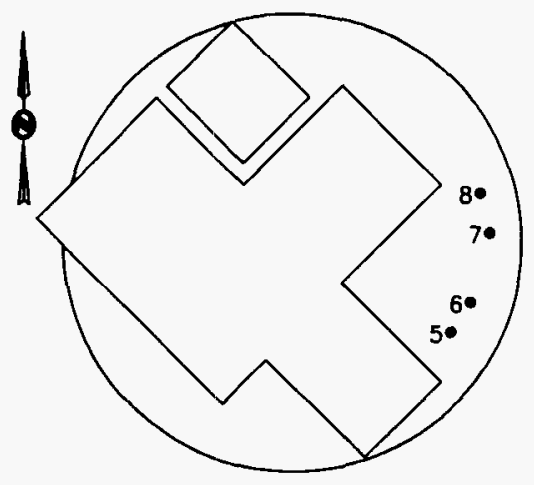

\begin{tabular}{|c|c|c|c|c|}
\hline \multirow{2}{*}{ RISER } & \multirow{2}{*}{ DESCRIPTION } & \multicolumn{2}{|c|}{ RESISTANCE(OHMS) } & \multirow{2}{*}{ COMMENTS } \\
\hline & & RISER & TOP & \\
\hline 5 & 4" REEL. & .58 & 7.45 & \\
\hline 6 & 12" TEMPERATURE & .52 & 2.24 & \\
\hline 7 & $12^{\prime \prime}$ OBSV PORT & 21.7 & 21.7 & U.G.R. \\
\hline 8 & 4" BREATHER FLTR & .56 & .56 & \\
\hline & & & & \\
\hline & & & & \\
\hline & & & & \\
\hline & & & & \\
\hline & & & & \\
\hline & & & & \\
\hline & & & & \\
\hline & & & & \\
\hline & & & & \\
\hline & & & & \\
\hline & & $\begin{array}{l}\text { TES } \\
\text { CAL }\end{array}$ & $\begin{array}{l}\text { UIP \# } \\
\text { UE DA }\end{array}$ & $\begin{array}{l}785-77-47-001 \\
3-12-97\end{array}$ \\
\hline
\end{tabular}


WHC-SD-WM-TR-034, REV. 0

\section{RISER RESISTANCE DATA SHEET}

TANK C-204

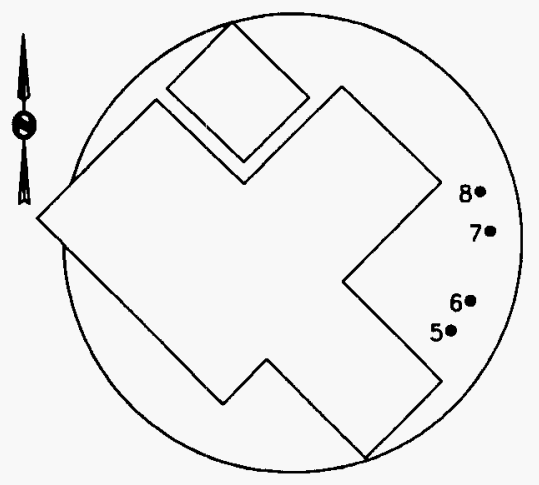

\begin{tabular}{|c|c|c|c|c|}
\hline \multirow{2}{*}{ RISER } & \multirow{2}{*}{ DESCRIPTION } & \multicolumn{2}{|c|}{ RESISTANCE(OHMS) } & \multirow{2}{*}{ COMMENTS } \\
\hline & & RISER & TOP & \\
\hline 5 & 4" REEL & .53 & $7.45(P)$ & PEDESTAL \\
\hline 6 & 12" TEMPERATURE & 24.3 & 24.3 & U.G.R. \\
\hline 7 & 12" OBSV PORT & 22.5 & 22.5 & U.G.R. \\
\hline 8 & 4" BREATHER FLTR & .52 & .61 & \\
\hline & & & & \\
\hline & & & & \\
\hline & & & & \\
\hline & & & & \\
\hline & & & & \\
\hline & & & & \\
\hline & & & & \\
\hline & & & & \\
\hline & & & & \\
\hline & & & & \\
\hline
\end{tabular}

TEST EQUIP \# 785-77-47-001

CALIB DUE DATE 3-12-97 


\section{RISER RESISTANCE DATA SHEET}

TANK S-101

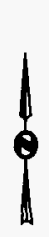

\begin{tabular}{|c|c|c|c|c|}
\hline \multirow{2}{*}{ RISER } & \multirow{2}{*}{ DESCRIPTION } & \multicolumn{2}{|c|}{ RESISTANCE(OHMS) } & \multirow{2}{*}{ COMMENTS } \\
\hline & & RISER & TOP & \\
\hline 2 & 4" LOW & .23 & & \\
\hline 3 & 4" ENRAF & .22 & .50 & \\
\hline 4 & 4" SPARE & .21 & .21 & \\
\hline 6 & 12" OBSV PORT & .24 & 3.79 & \\
\hline 7 & 12" EXHAUST DUCT & .21 & .22 & \\
\hline 8 & 12" PLUG & .22 & .30 & \\
\hline (11) & 4" SLUDGE MEAS & 31.3 & 31.4 & U.G.R. \\
\hline (14) & 4" TEMP PROBE & .21 & .21 & \\
\hline (16) & 4" BREATHER FLTR & 54.6 & 54.6 & U.G.R. \\
\hline $\mathrm{JB}$ & PROBE J-BOX & & 177.4 & \\
\hline PCS & PUMP CONTROL STA. & & 2.09 & \\
\hline EU & ENRAF UNISTRUT & & .49 & \\
\hline WF & WF ENCLOSURE & & .54 & \\
\hline
\end{tabular}

TEST EQUIP \# 785-77-47-001

CALIB DUE DATE 3-12-97 


\section{RISER RESISTANCE DATA SHEET}

\begin{tabular}{|c|c|c|c|c|}
\hline \multirow{2}{*}{ RISER } & \multirow{2}{*}{ DESCRIPTION } & \multicolumn{2}{|c|}{ RESISTANCE(OHMS) } & \multirow{2}{*}{ COMMENTS } \\
\hline & & RISER & TOP & \\
\hline 1 & 4" BREATHER FLTR & .18 & .18 & \\
\hline 2 & 4" ENRAF & .18 & .41 & \\
\hline 3 & 4" TEMP PROBE & .19 & .24 & \\
\hline 5 & $12 "$ LOW & .19 & & \\
\hline 6 & $12 "$ EXHAUST DUCT & .22 & .22 & \\
\hline 7 & $12^{\prime \prime}$ OBSV PORT & .22 & .20 & \\
\hline (11) & 4" SLUDGE MEAS & 36.0 & 36.0 & U.G.R. \\
\hline (14) & 4" SLUDGE MEAS & 48.6 & 48.5 & U.G.R. \\
\hline (16) & $4^{\prime \prime}$ SLUDGE MEAS & 27.6 & 27.8 & U.G.R. \\
\hline PCS & PUMP CONTROL STA & & 1.83 & \\
\hline JB1 & PROBE $\triangleleft-B O X$ & & 1.56 & \\
\hline $\mathrm{P} 2$ & $1 "$ PIPING & & .12 & \\
\hline & CONTINUED ON SHEET 2 & $\begin{array}{l}\text { TES } \\
\text { CAL }\end{array}$ & $\begin{array}{l}\text { EQUIP \# } \\
\text { DUE DA }\end{array}$ & $\begin{array}{l}785-77-47-001 \\
3-12-97\end{array}$ \\
\hline
\end{tabular}




\section{RISER RESISTANCE DATA SHEET}

TANK S-102 CONTINUED FROM SHEET 1

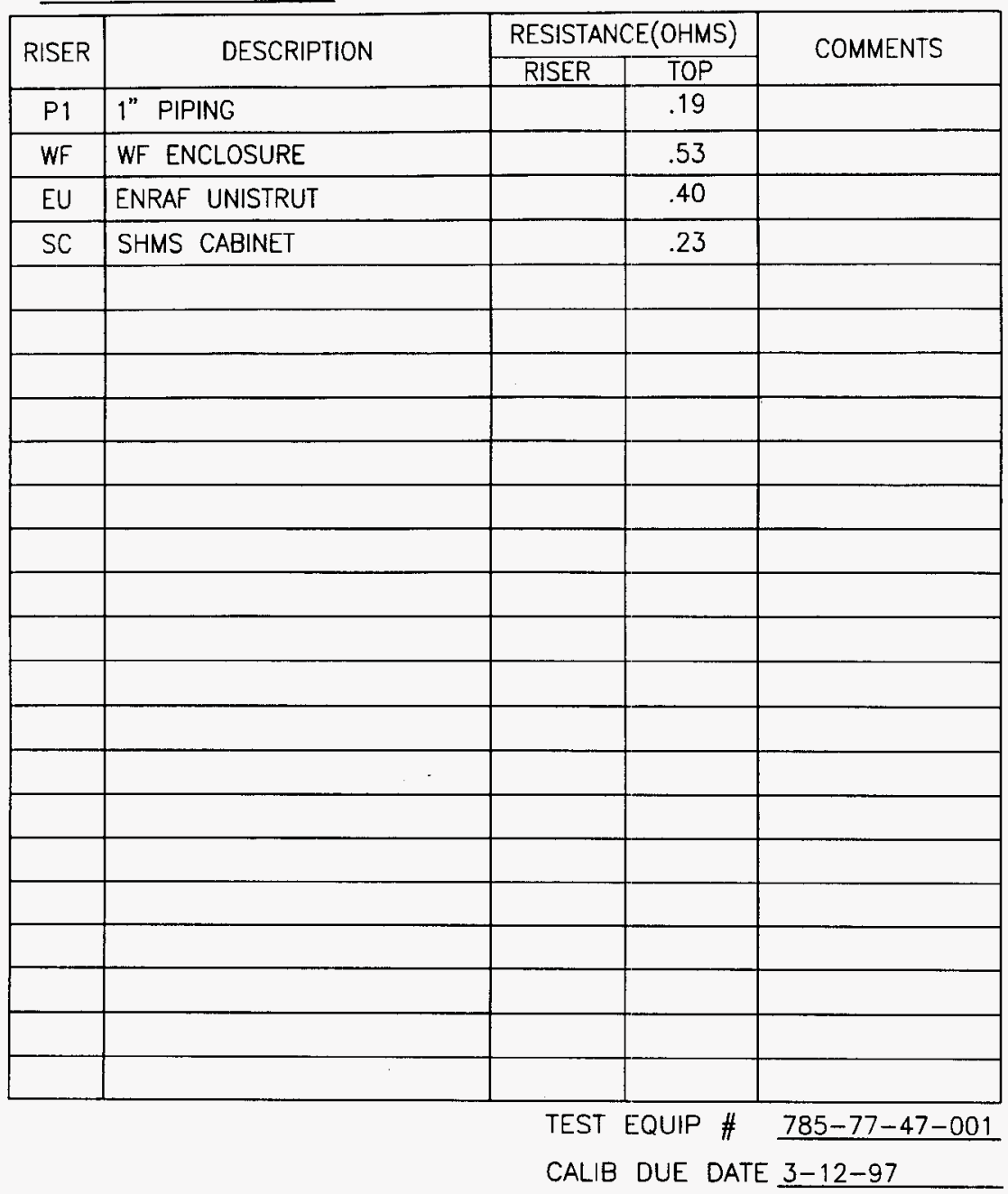




\section{RISER RESISTANCE DATA SHEET}

TANK S-103

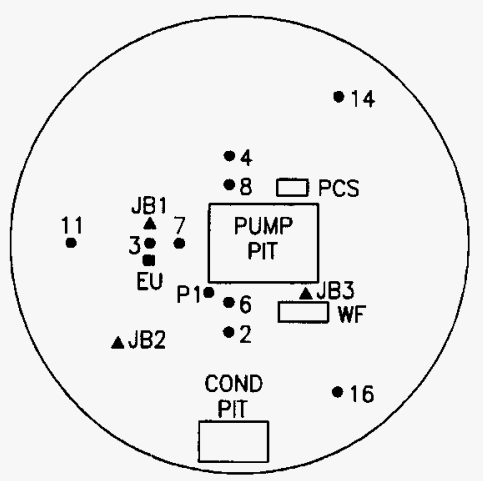

\begin{tabular}{|c|c|c|c|c|}
\hline \multirow{2}{*}{ RISER } & \multirow{2}{*}{ DESCRIPTION } & \multicolumn{2}{|c|}{ RESISTANCE(OHMS) } & \multirow{2}{*}{ COMMENTS } \\
\hline & & RISER & TOP & \\
\hline 2 & 4" LOW & .13 & & \\
\hline 3 & 4" ENRAF & .14 & .17 & \\
\hline 4 & 4" TEMP PROBE & .12 & .18 & \\
\hline 6 & $12^{\prime \prime}$ PLUG & .16 & .16 & \\
\hline 7 & 12" EXHAUST DUCT & .13 & .14 & \\
\hline 8 & 12" OBSV PORT & .12 & 1.26 & \\
\hline (11) & 4" SLUDGE MEAS & 37 & 37 & U.G.R. \\
\hline (14) & 4" BREATHER FLTR & 28.5 & 28.5 & U.G.R. \\
\hline (16) & 4" SLUDGE MEAS & 37.8 & 37.8 & U.G.R. \\
\hline PCS & PUMP CONTROL STA & & 1.85 & \\
\hline JB1 & PROBE J-BOX & & 3.42 & \\
\hline EU & ENRAF UNISTRUT & & .17 & \\
\hline JB2 & JUNCTION BOXES & & .27 & \\
\hline$P 1$ & PIPE & & $1.5 \mathrm{~K}$ & \\
\hline & CONTINUED ON SHEET 2 & $\begin{array}{l}\text { TEST } \\
\text { CALI }\end{array}$ & $\begin{array}{l}\text { QUIP \# } \\
\text { UE DAT }\end{array}$ & $\begin{array}{l}785-77-47-001 \\
3-12-97\end{array}$ \\
\hline
\end{tabular}




\section{RISER RESISTANCE DATA SHEET}

TANK S-103 CONTINUED FROM SHEET 1

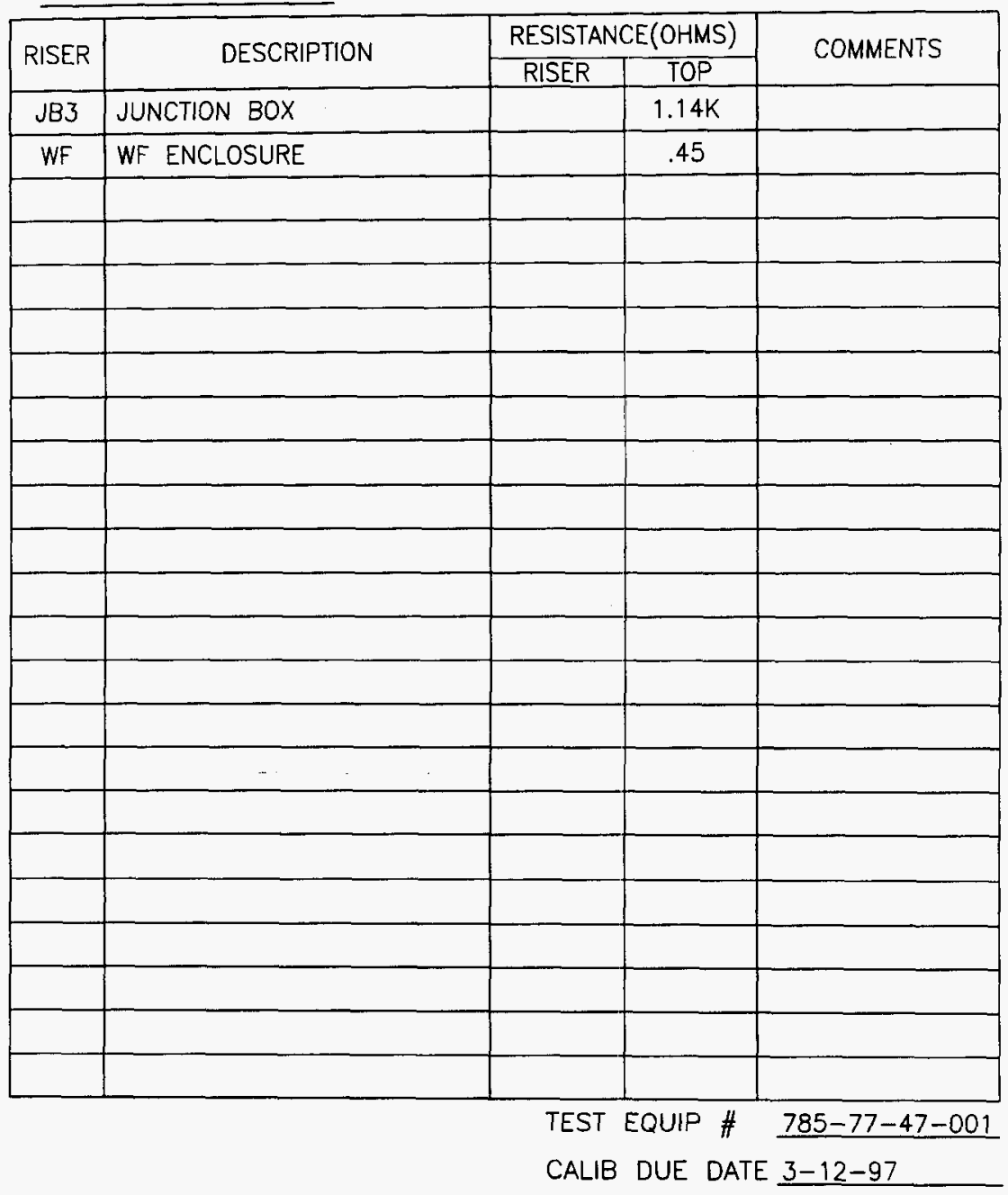




\section{RISER RESISTANCE DATA SHEET}

TANK S-104

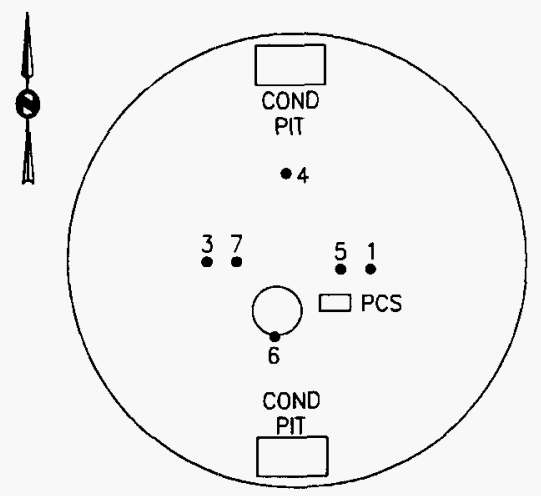

\begin{tabular}{|c|l|c|c|c|}
\hline \multirow{2}{*}{ RISER } & \multirow{2}{*}{ DESCRIPTION } & \multicolumn{2}{c|}{ RESISTANCE(OHMS) } & \multirow{2}{*}{ COMMENTS } \\
\cline { 3 - 4 } & & RISER & TOP & \multirow{2}{*}{} \\
\hline 1 & $4 "$ SPARE & .13 & .20 & \\
\hline 3 & $4 "$ BREATHER FLTR & .13 & .29 & \\
\hline 4 & $4 "$ TEMP PROBE & .15 & .29 & \\
\hline 5 & $12 "$ REEL & .13 & $1.78(\mathrm{P})$ & PEDESTAL \\
\hline 6 & $4 "$ SPARE & .14 & .14 & \\
\hline 7 & $12 "$ OBSV PORT & .12 & .21 & \\
\hline PCS & PUMP CONTROL STA & & .53 & \\
\hline & & & & \\
\hline & & & & \\
\hline & & & & \\
\hline
\end{tabular}

TEST EQUIP \# 785-77-47-001

CALIB DUE DATE $3-12-97$ 


\section{RISER RESISTANCE DATA SHEET}

TANK S-105

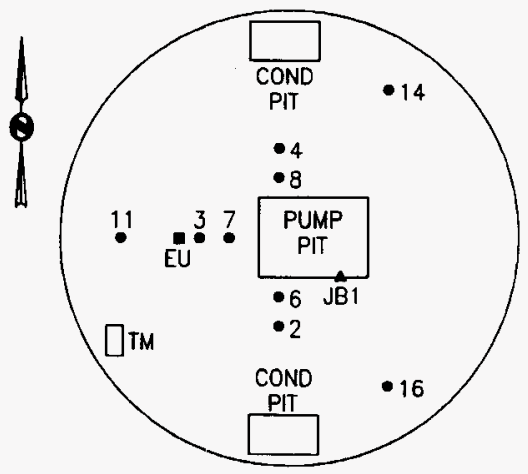

\begin{tabular}{|c|l|c|c|c|}
\hline \multirow{2}{*}{ RISER } & \multirow{2}{*}{ DESCRIPTION } & \multicolumn{2}{c|}{ RESISTANCE(OHMS) } & \multirow{2}{*}{ COMMENTS } \\
\cline { 3 - 4 } & & RISER & TOP & \\
\hline 2 & $4 "$ SAMPLE LINE & .16 & .16 & \\
\hline 3 & $4 "$ ENRAF & .15 & .38 & \\
\hline 4 & $4 "$ TEMP PROBE & .16 & .15 & \\
\hline 6 & $12 "$ SPARE & .17 & .18 & \\
\hline 7 & $12 "$ EXHAUST DUCT & .16 & .16 & \\
\hline 8 & $12 "$ OBSV PORT & .16 & .46 & \\
\hline (11) & $4 "$ SLUDGE MEAS & 29.7 & 29.7 & U.G.R. \\
\hline (14) & $4 "$ LOW & 41.8 & & U.G.R. \\
\hline (16) & $4 "$ BREATHER FLTR & 32.8 & 32.8 & U.G.R. \\
\hline EU & ENRAF UNISTRUT & & .54 & \\
\hline JB1 & JUNCTION BOX & & .47 & \\
\hline TM & TMACS & & .16 & \\
\hline
\end{tabular}

TEST EQUIP \# 785-77-47-001

CALIB DUE DATE 3-12-97 


\section{RISER RESISTANCE DATA SHEET}

TANK S-106

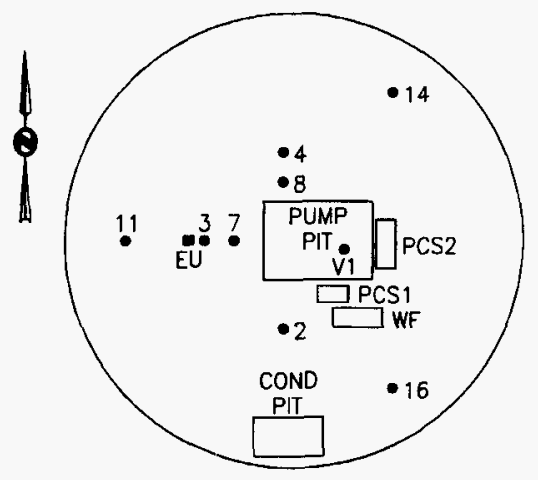

\begin{tabular}{|c|c|c|c|c|}
\hline \multirow{2}{*}{ RISER } & \multirow{2}{*}{ DESCRIPTION } & \multicolumn{2}{|c|}{ RESISTANCE(OHMS) } & \multirow{2}{*}{ COMMENTS } \\
\hline & & RISER & TOP & \\
\hline 2 & 4" TEMP PROBE & .16 & .16 & \\
\hline 3 & $4^{\prime \prime}$ ENRAF & .18 & .17 & \\
\hline 4 & 4" LOW & .18 & & \\
\hline 7 & 12" EXHAUST DUCT & .24 & .40 & \\
\hline 8 & 12" OBSV PORT & .17 & 1.45 & \\
\hline (11) & 4" SLUDGE MEAS & 37.5 & 37.5 & U.G.R. \\
\hline (14) & 4" SLUDGE MEAS & 36.8 & 37.8 & U.G.R. \\
\hline (16) & 4" BREATHER FLTR & 35.3 & 35.3 & U.G.R. \\
\hline$\overline{E U}$ & ENRAF UNISTRUT & & .16 & \\
\hline $\mathrm{V} 1$ & VALVE HANDLES & & & \\
\hline PCS2 & PUMP CONTROL SKID & & .18 & \\
\hline PCS1 & PUMP CONTROL STA & & .14 & \\
\hline WF & WF ENCLOSURE & & .32 & \\
\hline
\end{tabular}

TEST EQUIP \# 785-77-47-001

CALIB DUE DATE 3-12-97 
RISER RESISTANCE DATA SHEET

TANK S-107
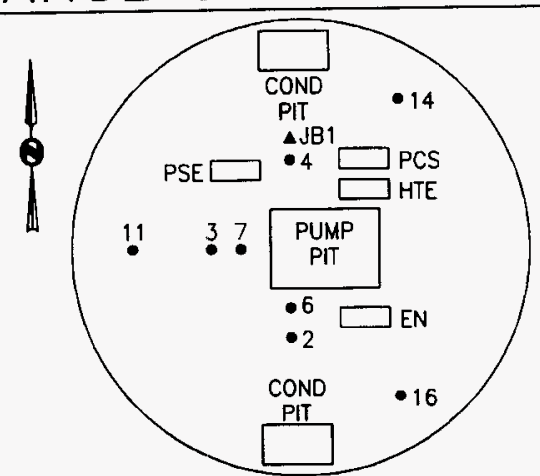

\begin{tabular}{|c|c|c|c|c|}
\hline \multirow{2}{*}{ RISER } & \multirow{2}{*}{ DESCRIPTION } & \multicolumn{2}{|c|}{ RESISTANCE(OHMS) } & \multirow{2}{*}{ COMMENTS } \\
\hline & & RISER & TOP & \\
\hline 2 & 4" BREATHER & .11 & .17 & \\
\hline 3 & 4" ENRAF & .12 & .66 & \\
\hline 4 & 4" TEMP PROBE & .11 & .15 & \\
\hline 6 & 12" OBSV PORT & .11 & .11 & \\
\hline 7 & $12^{\prime \prime}$ SPARE & .11 & .12 & \\
\hline (11) & 4" SLUDGE MEAS & 29.3 & 29.3 & U.G.R. \\
\hline (14) & 4" SLUDGE MEAS & 34.1 & 122.8 & U.G.R. \\
\hline (16) & 4" SPARE & 29.4 & 29.3 & U.G.R. \\
\hline PSE & $\begin{array}{l}\text { POWER SUPPLY \& } \\
\text { FLOW METER ENCL }\end{array}$ & & .23 & \\
\hline JB1 & PROBE J-BOX & & .22 & \\
\hline PCS & PUMP CONTROL SKID & & .23 & \\
\hline HTE & $\begin{array}{l}\text { HEAT TRACE \& } \\
\text { PUMP CONTROL ENCL }\end{array}$ & & .31 & \\
\hline EN & ENCLOSURE & & .57 & \\
\hline & & $\begin{array}{l}\text { TEST } \\
\text { CALI }\end{array}$ & $\begin{array}{l}\text { QUIP \# } \\
\text { DUE DA }\end{array}$ & $\frac{785-77-47-001}{3-12-97}$ \\
\hline
\end{tabular}




\section{RISER RESISTANCE DATA SHEET}

TANK S-108

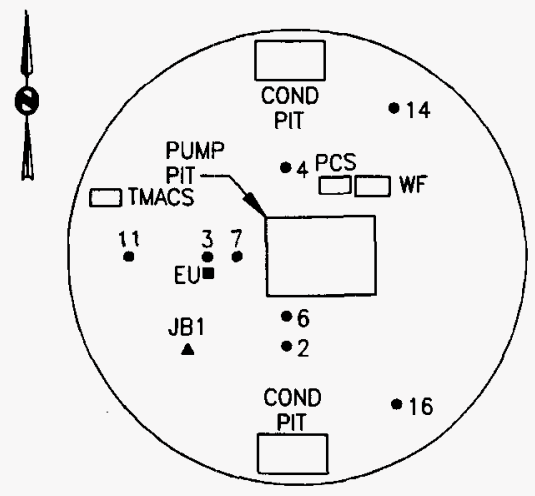

\begin{tabular}{|c|l|c|c|c|}
\hline \multirow{2}{*}{ RISER } & \multirow{2}{*}{ DESCRIPTION } & \multicolumn{2}{c|}{ RESISTANCE(OHMS) } & \multirow{2}{*}{ COMMENTS } \\
\cline { 3 - 4 } & & RISER & TOP & \\
\hline 2 & $4 "$ REEL & .22 & $5.06 \mathrm{~K}$ & \\
\hline 3 & $4 "$ ENRAF & .14 & .87 & \\
\hline 4 & $4 "$ TEMP PROBE & .14 & .15 & \\
\hline 6 & $12 "$ SPARE & .14 & .15 & \\
\hline 7 & $12 "$ OBSV PORT & .14 & 667 & \\
\hline$(11)$ & $4 "$ SLUDGE MEAS & 38.4 & 38.4 & U.G.R. \\
\hline (14) & $4^{\prime \prime}$ BREATHER FLTR & 47.1 & 47.2 & U.G.R. \\
\hline (16) & 4" LOW & 35.6 & & U.G.R. \\
\hline PCS & PUMP CONTROL STA & & .20 & \\
\hline WF & WF ENCLOSURE & & .20 & \\
\hline
\end{tabular}

CONTINUED ON SHEET 2

TEST EQUIP \# 785-77-47-001

CALIB DUE DATE 3-12-97 


\section{RISER RESISTANCE DATA SHEET}

TANK S-108 CONTINUED FroM SHEET 1

\begin{tabular}{|c|c|c|c|c|}
\hline \multirow{2}{*}{ RISER } & \multirow{2}{*}{ DESCRIPTION } & \multicolumn{2}{|c|}{ RESISTANCE(OHMS) } & \multirow{2}{*}{ COMMENTS } \\
\hline & & RISER & TOP & \\
\hline & TMACS & & .11 & \\
\hline EU & ENRAF UNISTRUT & & .91 & \\
\hline JB1 & FIC JUNCTION BOXES & & .45 & \\
\hline & & & & \\
\hline & & & & \\
\hline & & & & \\
\hline & & & & \\
\hline & & & & \\
\hline & & & & \\
\hline & & & & \\
\hline & & & & \\
\hline & & & & \\
\hline & & & & \\
\hline & & & & \\
\hline & & & & \\
\hline & & & & \\
\hline & & & & \\
\hline & & & & \\
\hline & & & & \\
\hline & & & & \\
\hline & & & & \\
\hline & & & & \\
\hline & & & & \\
\hline & & TES & UIP \# & $85-77-47-001$ \\
\hline & & CAL & UE DA & $-12-97$ \\
\hline
\end{tabular}




\section{RISER RESISTANCE DATA SHEET}

TANK S-109

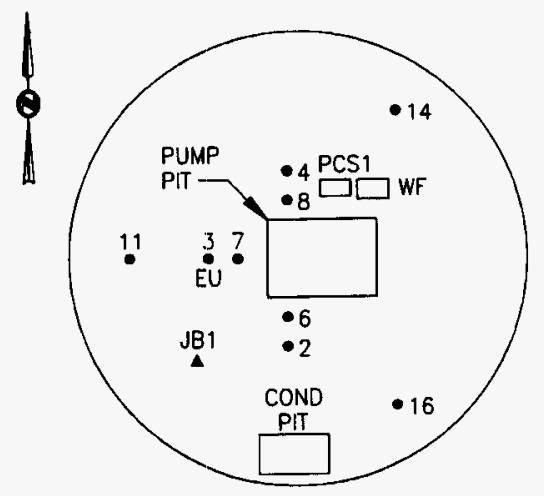

\begin{tabular}{|c|c|c|c|c|}
\hline \multirow{2}{*}{ RISER } & \multirow{2}{*}{ DESCRIPTION } & \multicolumn{2}{|c|}{ RESISTANCE(OHMS) } & \multirow{2}{*}{ COMMENTS } \\
\hline & & RISER & TOP & \\
\hline 2 & 4" BREATHER FLTR & .12 & .44 & \\
\hline 3 & 4" ENRAF & .11 & .29 & \\
\hline 4 & 4" TEMP PROBE & .12 & .12 & \\
\hline 6 & 12" OBSV PORT & .12 & .13 & \\
\hline 7 & 12" SPARE & .11 & .12 & \\
\hline 8 & $12 "$ LOW & .13 & & \\
\hline (11) & 4" SLUDGE MEAS & 42.1 & 42.1 & U.G.R. \\
\hline (14) & 4" SLUDGE MEAS & 32.4 & 32.4 & U.G.R. \\
\hline (16) & $4^{n}$ SLUDGE MEAS & 33.4 & 33.4 & U.G.R. \\
\hline JB1 & JUNCTION BOXES & & 2.92 & \\
\hline
\end{tabular}

CONTINUED ON SHEET 2

TEST EQUIP \# 785-77-47-001 CALIB DUE DATE 3-12-97 


\section{RISER RESISTANCE DATA SHEET}

TANK S-109 CONTINUED FROM SHEET 1

\begin{tabular}{|c|c|c|c|c|}
\hline \multirow{2}{*}{ RISER } & \multirow{2}{*}{ DESCRIPTION } & \multicolumn{2}{|c|}{ RESISTANCE(OHMS) } & \multirow{2}{*}{ COMMENTS } \\
\hline & & RISER & TOP & \\
\hline$\overline{E U}$ & ENRAF UNISTRUT & & 1.22 & \\
\hline PCS1 & PUMP CONTROL STA & & .19 & \\
\hline WF & WF ENCLOSURE & & .19 & \\
\hline & & & & \\
\hline & & & & \\
\hline & & & & \\
\hline & & & & \\
\hline & & & & \\
\hline & & & & \\
\hline & & & & \\
\hline & & & & \\
\hline & & & & \\
\hline & & & & \\
\hline & & & & \\
\hline & & & & \\
\hline & & & & \\
\hline & & & & \\
\hline & & & & \\
\hline & & & & \\
\hline & & & & \\
\hline & & & & \\
\hline & & & & \\
\hline & & & & \\
\hline & & $\begin{array}{l}\text { TEST } \\
\text { CALI }\end{array}$ & $\begin{array}{l}\text { QUIP \# } \\
\text { UE DAT }\end{array}$ & $\begin{array}{l}85-77-47-001 \\
-12-97\end{array}$ \\
\hline
\end{tabular}




\section{RISER RESISTANCE DATA SHEET}

TANK S-110

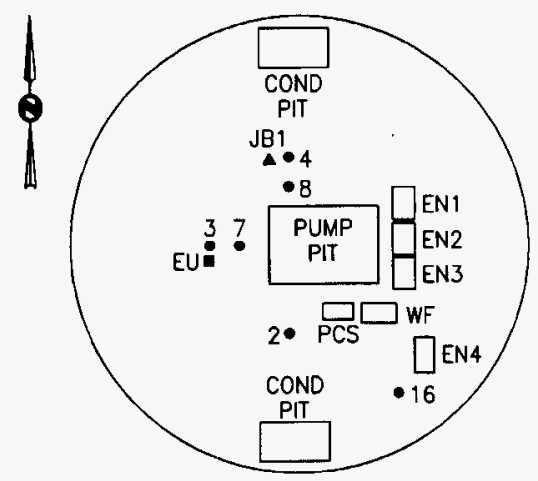

\begin{tabular}{|c|c|c|c|c|}
\hline \multirow{2}{*}{ RISER } & \multirow{2}{*}{ DESCRIPTION } & \multicolumn{2}{|c|}{ RESISTANCE(OHMS) } & \multirow{2}{*}{ COMMENTS } \\
\hline & & RISER & TOP & \\
\hline 2 & 4" LOW & .17 & & \\
\hline 3 & 4" ENRAF & .11 & .28 & \\
\hline 4 & 4" TEMP PROBE & .11 & .11 & \\
\hline 7 & 12" OBSV PORT & .11 & .12 & \\
\hline 8 & 12" SPARE & .14 & .14 & \\
\hline (16) & 4" BREATHER FLTR & .18 & .18 & \\
\hline JB1 & PROBE J-BOX & & .28 & \\
\hline EU & ENRAF UNISTRUT & & .27 & \\
\hline EN1 & ENCLOSURE & & .16 & \\
\hline EN2 & ENCLOSURE & & .16 & \\
\hline EN3 & ENCLOSURE & & .16 & \\
\hline & CONTINUED ON SHEET 2 & $\begin{array}{l}\text { TEST } \\
\text { CALI }\end{array}$ & $\begin{array}{l}\text { UIP \# } \\
\text { JE DAT }\end{array}$ & $\begin{array}{l}785-77-47-001 \\
3-12-97\end{array}$ \\
\hline
\end{tabular}




\section{RISER RESISTANCE DATA SHEET}

TANK $S-110$ CONTINUED FROM SHEET 1

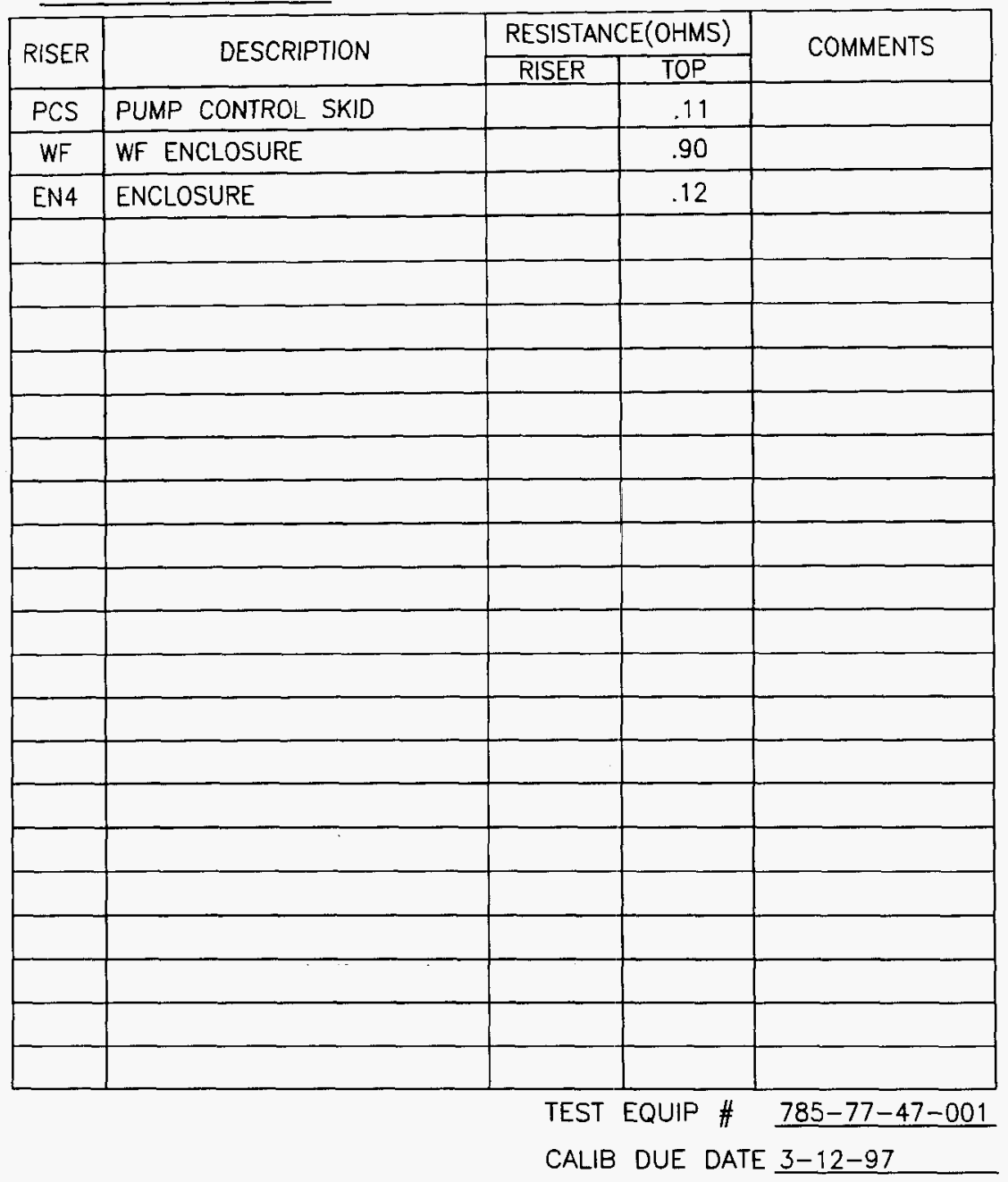




\section{RISER RESISTANCE DATA SHEET}

TANK S-111

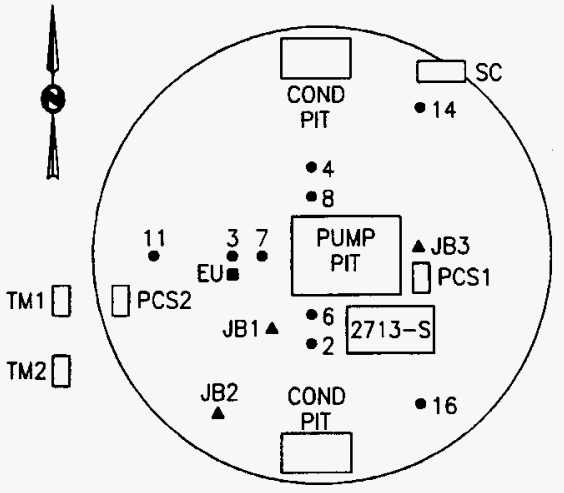

\begin{tabular}{|c|c|c|c|c|}
\hline \multirow{2}{*}{ RISER } & \multirow{2}{*}{ DESCRIPTION } & \multicolumn{2}{|c|}{ RESISTANCE(OHMS) } & \multirow{2}{*}{ COMMENTS } \\
\hline & & RISER & TOP & \\
\hline 2 & $4^{\prime \prime} \mathrm{FIC}$ & .12 & .11 & \\
\hline 3 & 4" ENRAF & .13 & .21 & \\
\hline 4 & 4" TEMP PROBE & .11 & .13 & \\
\hline 6 & 12" SPARE & .11 & .11 & \\
\hline 7 & 12" SPARE & .11 & .12 & \\
\hline 8 & 12" OBSV PORT & .10 & .14 & \\
\hline (11) & 4" SPARE & .12 & .29 & \\
\hline (14) & 4" BREATHER FLTR & .17 & .18 & \\
\hline (16) & 4" LOW & 29.7 & & U.G.R. \\
\hline $\mathrm{SC}$ & SHMS CABINET & & .38 & \\
\hline
\end{tabular}

CONTINUED ON SHEET 2

TEST EQUIP \# 785-77-47-001

CALIB DUE DATE 3-12-97 


\section{RISER RESISTANCE DATA SHEET}

TANK S-111 CONTINUED FROM SHEET 1

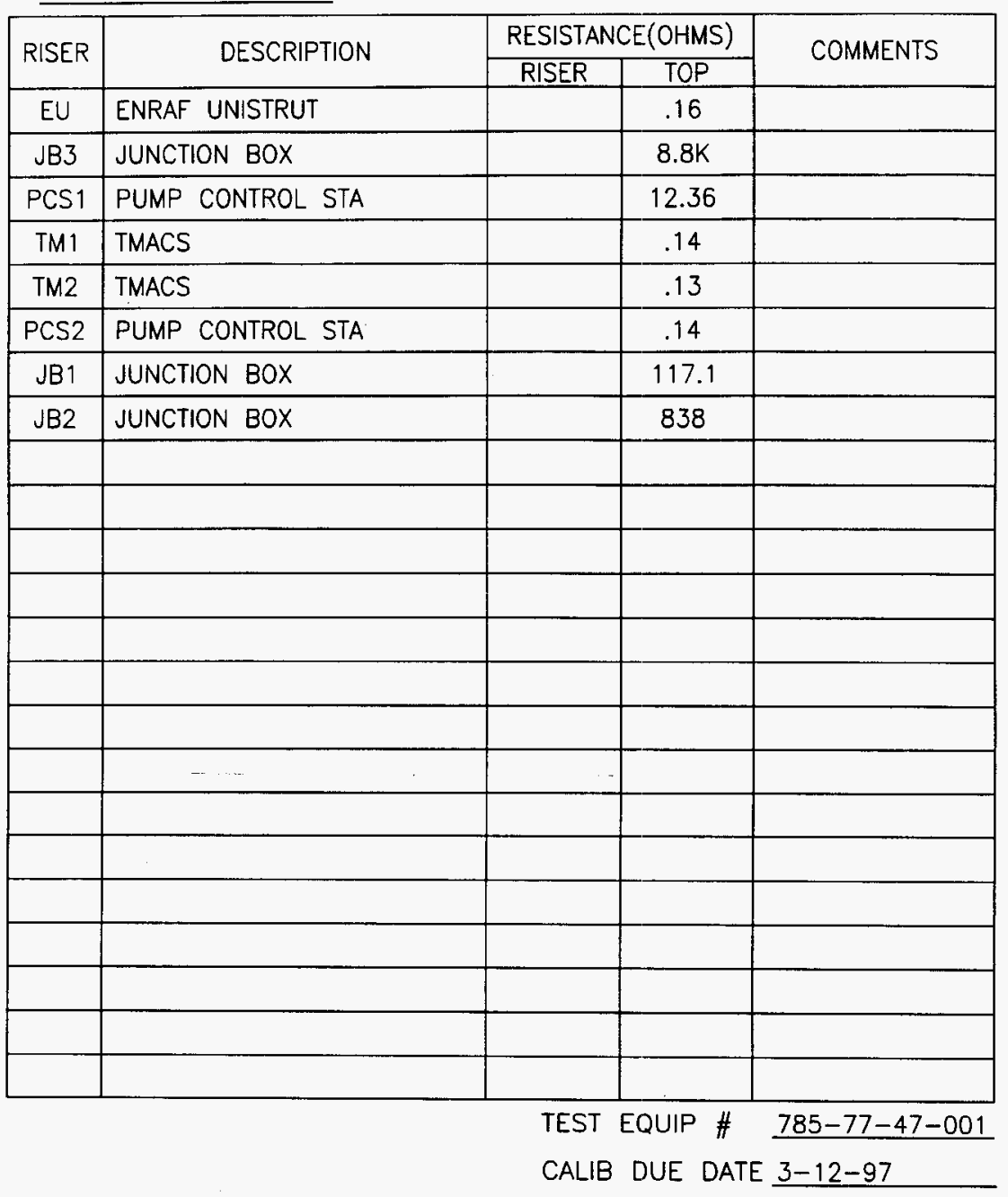




\section{RISER RESISTANCE DATA SHEET}

TANK S-112

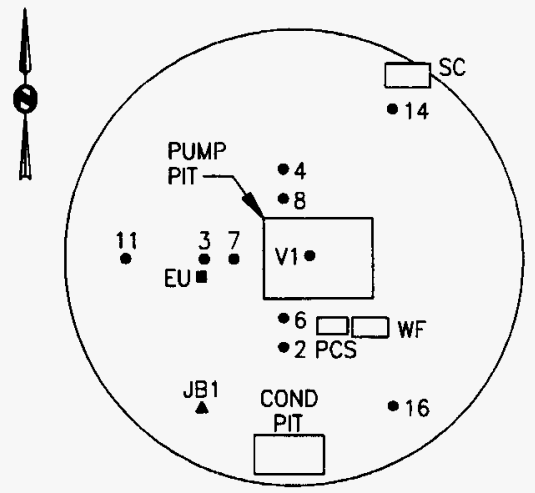

\begin{tabular}{|c|c|c|c|c|}
\hline \multirow{2}{*}{ RISER } & \multirow{2}{*}{ DESCRIPTION } & \multicolumn{2}{|c|}{ RESISTANCE(OHMS) } & \multirow{2}{*}{ COMMENTS } \\
\hline & & RISER & TOP & \\
\hline 2 & 4" SPARE & .11 & 52.9 & \\
\hline 3 & 4" FIC & .12 & .36 & \\
\hline 4 & 4" TEMP PROBE & .12 & .11 & \\
\hline 6 & 12" OBSV PORT & .11 & .17 & \\
\hline 7 & 12" SPARE & .10 & .25 & \\
\hline 8 & 12" LOW & .11 & .11 & \\
\hline (11) & 4" SLUDGE MEAS & 27.3 & 27.3 & U.G.R. \\
\hline (14) & 4" BREATHER FLTR & .20 & .20 & \\
\hline (16) & 4" SLUDGE MEAS & 29.8 & $3.3 \mathrm{~K}$ & U.G.R. \\
\hline $\mathrm{SC}$ & SHMS CABINET & & .12 & \\
\hline & CONTINUED ON SHEET 2 & $\begin{array}{l}\text { TES } \\
\text { CAL }\end{array}$ & $\begin{array}{l}\text { UIP \# } \\
\text { UE DA }\end{array}$ & $\frac{785-77-47-001}{3-12-97}$ \\
\hline
\end{tabular}




\section{RISER RESISTANCE DATA SHEET}

TANK S-112 CONTINUED FROM SHEEt 1

\begin{tabular}{|c|c|c|c|c|}
\hline \multirow{2}{*}{ RISER } & \multirow{2}{*}{ DESCRIPTION } & \multicolumn{2}{|c|}{ RESISTANCE(OHMS) } & \multirow{2}{*}{ COMMENTS } \\
\hline & & RISER & TOP & \\
\hline$E U$ & ENRAF UNISTRUT & & .75 & \\
\hline$V_{1}$ & VALVE HANDLE & & 930 & \\
\hline PCS & PUMP CONTROL STA & & .28 & \\
\hline WF & WF ENCLOSURE & & .24 & \\
\hline JB1 & JUNCTION BOXES & & .23 & \\
\hline & & & & \\
\hline & & & & \\
\hline & & & & \\
\hline & & & & \\
\hline & & & & \\
\hline & & & & \\
\hline & & & & \\
\hline & & & & \\
\hline & & & & \\
\hline & & & & \\
\hline & & & & \\
\hline & & & & \\
\hline & & & & \\
\hline & & & & \\
\hline & & & & \\
\hline & & & & \\
\hline & & & & \\
\hline & & & & \\
\hline
\end{tabular}

TEST EQUIP \# 785-77-47-001 CALIB DUE DATE $\underline{3-12-97}$ 


\section{RISER RESISTANCE DATA SHEET}
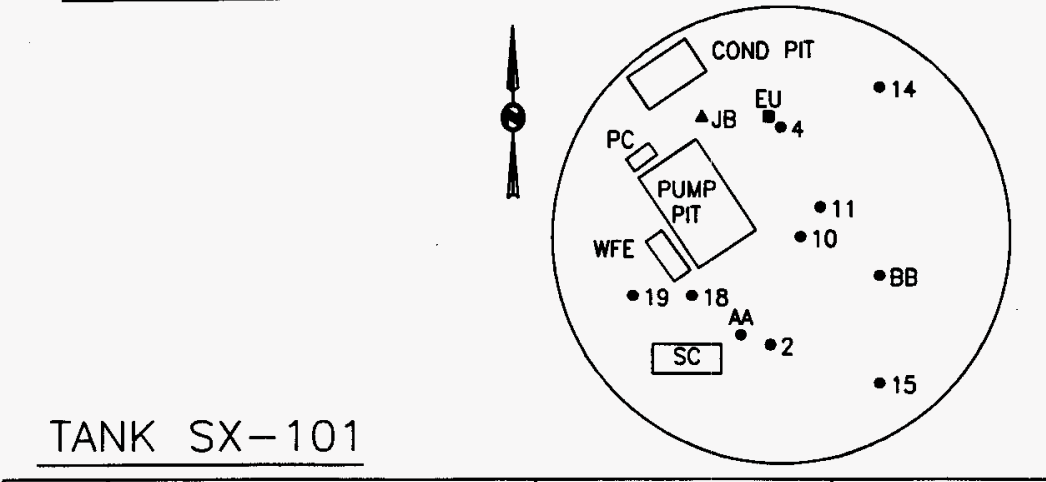

\begin{tabular}{|c|c|c|c|c|}
\hline \multirow{2}{*}{ RISER } & \multirow{2}{*}{ DESCRIPTION } & \multicolumn{2}{|c|}{ RESISTANCE(OHMS) } & \multirow{2}{*}{ COMMENTS } \\
\hline & & RISER & TOP & \\
\hline 2 & $4^{n}$ SPARE & .13 & .13 & \\
\hline 4 & $4^{\prime \prime}$ ENRAF & .12 & .67 & \\
\hline 10 & $3^{\prime \prime}$ SPARE & .13 & 9.55 & \\
\hline 11 & 8" FLANGE & .16 & .18 & \\
\hline 14 & 4" FIBERGLASS LOW & 37.8 & & U.G.R. \\
\hline (15) & 4" TEMP PROBE & .31 & .33 & \\
\hline & TEMP STAND & & .29 & \\
\hline 18 & 10"BREATHER FLTR & .38 & .41 & \\
\hline (19) & 12" OBSV PORT & 38.9 & 47.3 & U.G.R. \\
\hline AA & VALVE STAND & & .15 & \\
\hline (83) & 6" SPARE & 32.5 & 32.5 & U.G.R. \\
\hline EU & ENRAF UNISTRUT & & .66 & \\
\hline $\mathrm{JB}$ & $\mathrm{J} \mathrm{BOX}$ & & $1.196 \mathrm{~K}$ & \\
\hline$P C$ & PUMP CONTROL & & .29 & \\
\hline $\mathrm{SC}$ & SHMS CABINET & & .24 & \\
\hline \multicolumn{2}{|c|}{ CONTINUED NEXT SHEET } & \multicolumn{2}{|c|}{ TEST EQUIP \# } & $\begin{aligned} & \frac{785-77-47-001}{3-12-97} \\
= & 3-12\end{aligned}$ \\
\hline
\end{tabular}




\section{RISER RESISTANCE DATA SHEET}

\section{TANK SX-101 CONTINUED}

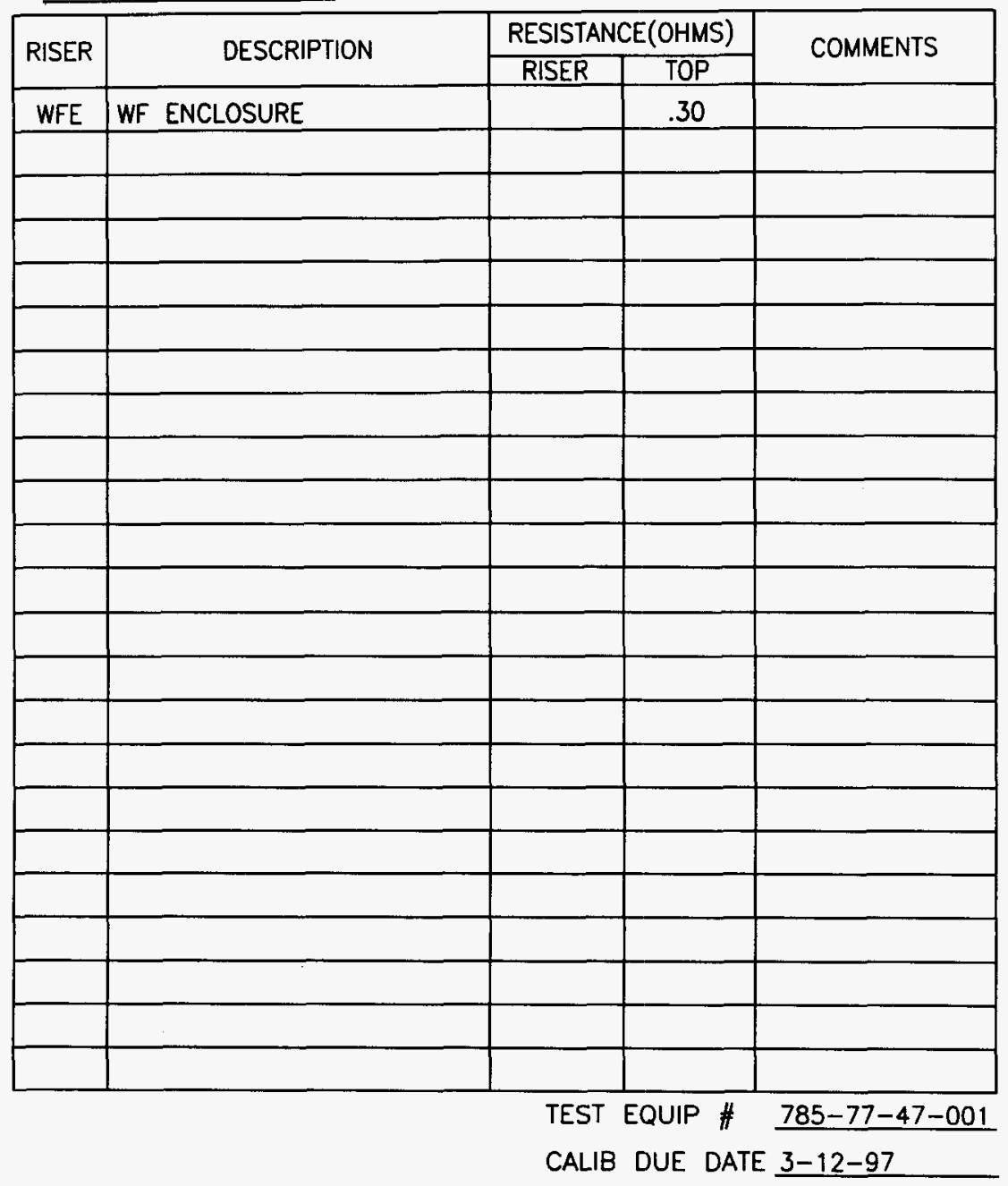




\section{RISER RESISTANCE DATA SHEET}

TANK SX-102

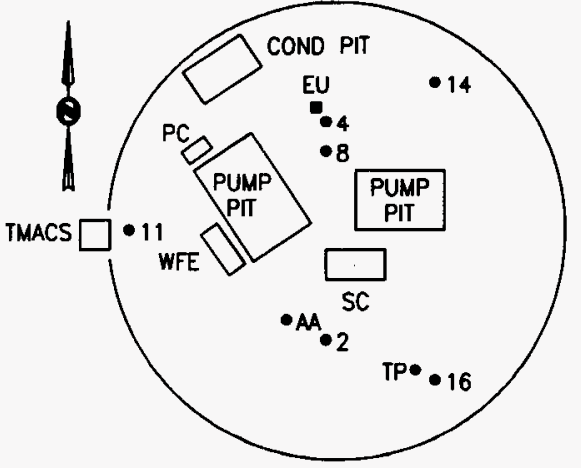

\begin{tabular}{|c|c|c|c|c|}
\hline \multirow{2}{*}{ RISER } & \multirow{2}{*}{ DESCRIPTION } & \multicolumn{2}{|c|}{ RESISTANCE(OHMS) } & \multirow{2}{*}{ COMMENTS } \\
\hline & & RISER & TOP & \\
\hline 2 & 4" BREATHER FLTR & .13 & .21 & \\
\hline 4 & 4" ENRAF & .17 & .49 & \\
\hline 8 & $12^{\prime \prime}$ OBSV PORT & .12 & .28 & \\
\hline (11) & 4" SPARE & 27.2 & 27.1 & U.G.R. \\
\hline (14) & 4" FIBERGLASS LOW & 23.3 & & U.G.R. \\
\hline (16) & 4" TEMP PROBE & 29.1 & .21 & U.G.R./G.T.W. \\
\hline$A A$ & VALVE STAND & & .16 & \\
\hline EU & ENRAF UNISTRUT & & .49 & \\
\hline $\mathrm{PC}$ & PUMP CONTROL & & .28 & \\
\hline \multirow[t]{2}{*}{$\mathrm{SC}$} & SHMS CABINET & & .14 & \\
\hline & TMACS & & .18 & \\
\hline TP & TEMP STAND & & .30 & \\
\hline WFE & WF ENCLOSURE & & .31 & \\
\hline & & & & \\
\hline & & & & \\
\hline & & $\begin{array}{l}\text { TES } \\
\text { CALI }\end{array}$ & $\begin{array}{l}\text { UIP \# } \\
\text { UE DAT }\end{array}$ & $\begin{array}{l}785-77-47-001 \\
3-12-97\end{array}$ \\
\hline
\end{tabular}




\section{RISER RESISTANCE DATA SHEET}

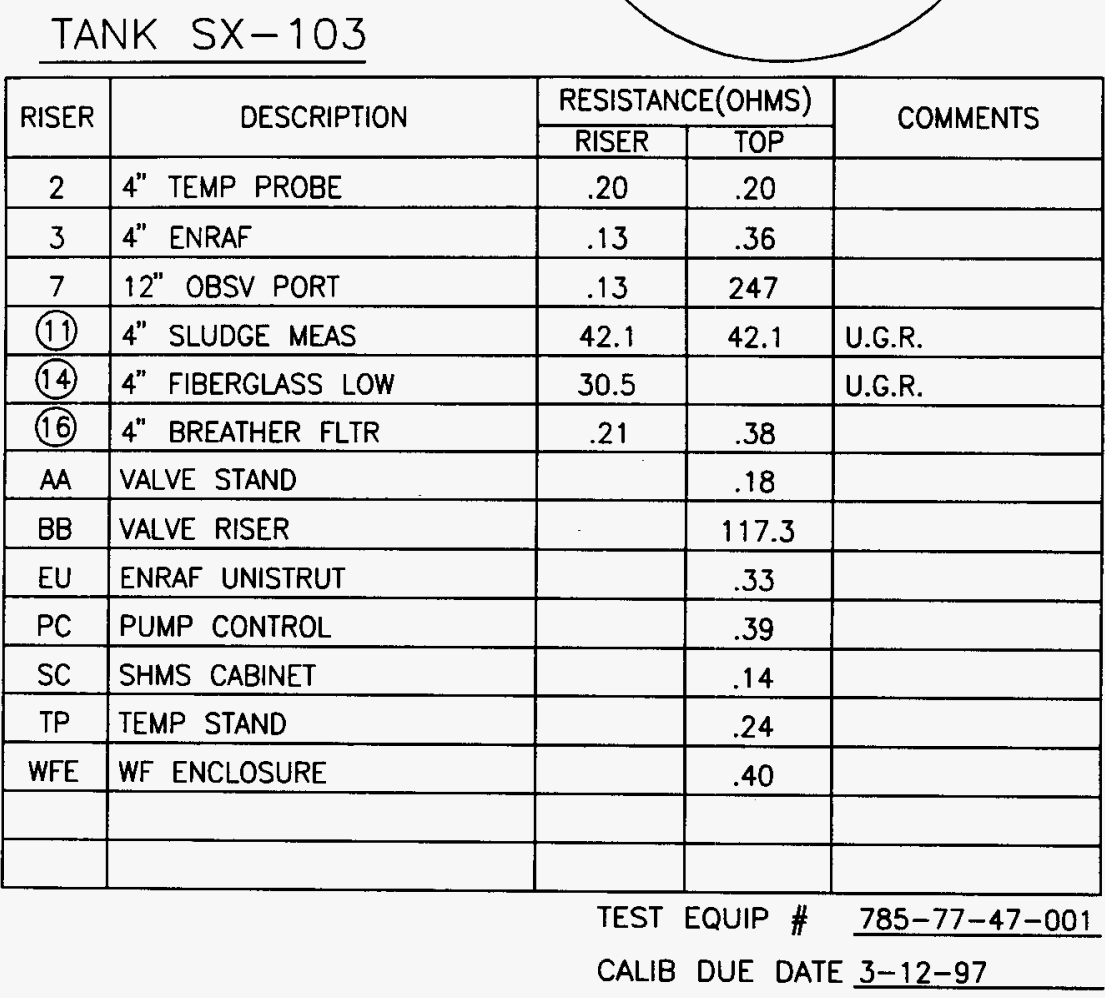


WHC-SD-WM-TR-034, REV. 0

\section{RISER RESISTANCE DATA SHEET}

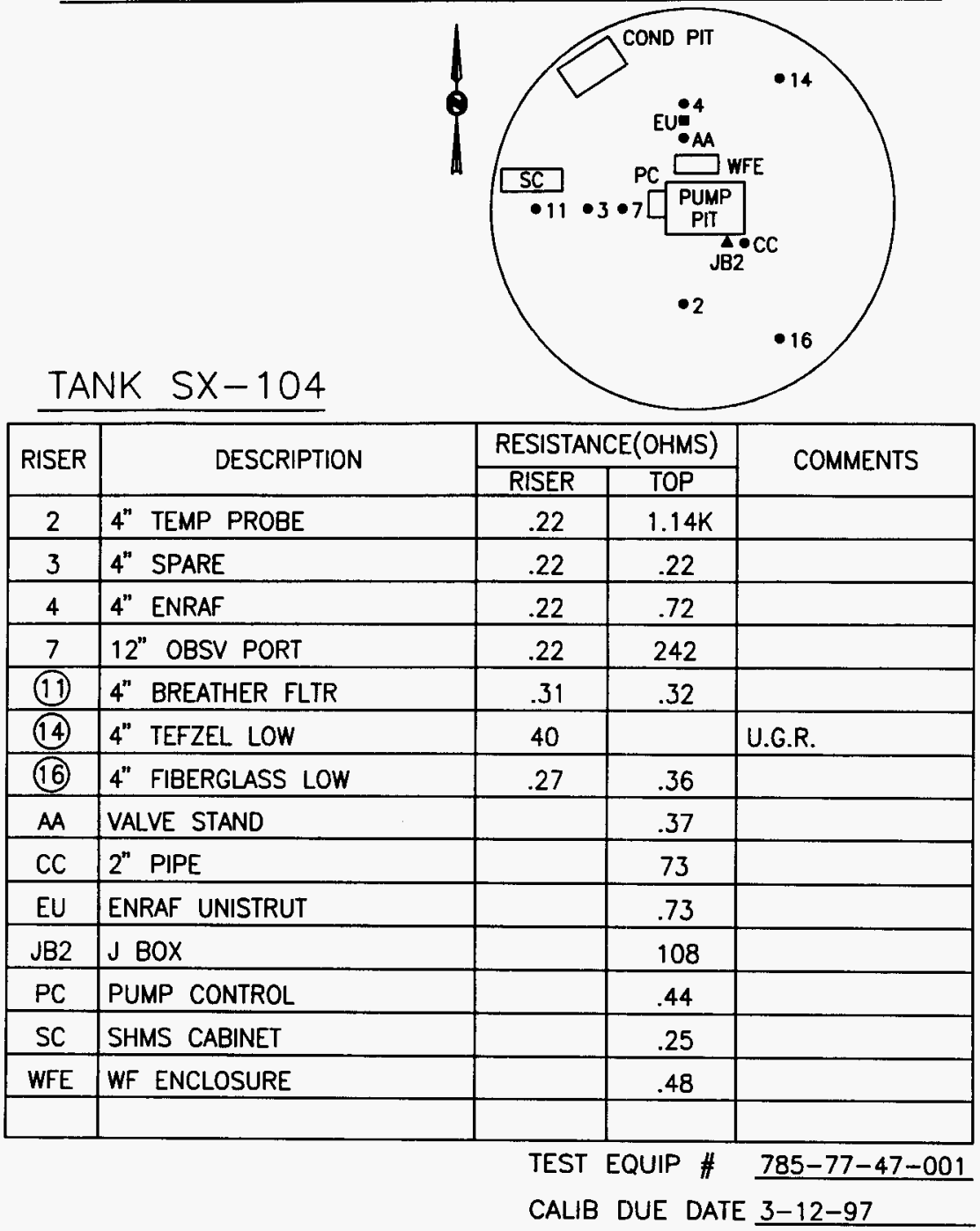


WHC-SD-WM-TR-034, REV. 0

\section{RISER RESISTANCE DATA SHEET}

\begin{tabular}{|c|c|c|c|c|}
\hline \multirow{2}{*}{ RISER } & \multirow{2}{*}{ DESCRIPTION } & \multicolumn{2}{|c|}{ RESISTANCE(OHMS) } & \multirow{2}{*}{ COMMENTS } \\
\hline & & RISER & TOP & \\
\hline 2 & 4" TEMP PROBE & .22 & .22 & \\
\hline 3 & 4" SPARE & .22 & 752 & \\
\hline 4 & 4" ENRAF & .21 & .56 & \\
\hline 6 & 12" OBSV PORT & .21 & .48 & \\
\hline 7 & $12^{n} \mathrm{BM}$ & .21 & 122 & \\
\hline (11) & 4" BREATHER FLTR & .34 & .34 & \\
\hline (12) & 4" SPARE & 2.16 & 2.17 & \\
\hline (14) & 4" SPARE & 41 & 41 & U.G.R. \\
\hline (15) & $2.5^{\prime \prime}$ SPARE & 40 & 40 & U.G.R. \\
\hline (16) & 4" FIBERGLASS LOW & 48 & & U.G.R. \\
\hline AA & VALVE STAND & & .26 & \\
\hline $\mathrm{BB}$ & VALVE HANDLE & & OPEN & \\
\hline $\mathrm{CC}$ & $2^{\prime \prime}$ PIPE & & 262 & \\
\hline COB1 & CLEAN OUT BOX & & .21 & \\
\hline EU & ENRAF UNISTRUT & & .56 & \\
\hline \multicolumn{2}{|c|}{ CONTINUED NEXT SHEET } & \multicolumn{3}{|c|}{$\begin{array}{l}\text { TEST EQUIP \# } 785-77-47-001 \\
\text { CALIB DUE DATE } 3-12-97\end{array}$} \\
\hline
\end{tabular}




\section{RISER RESISTANCE DATA SHEET}

TANK SX-105 CONTINUED

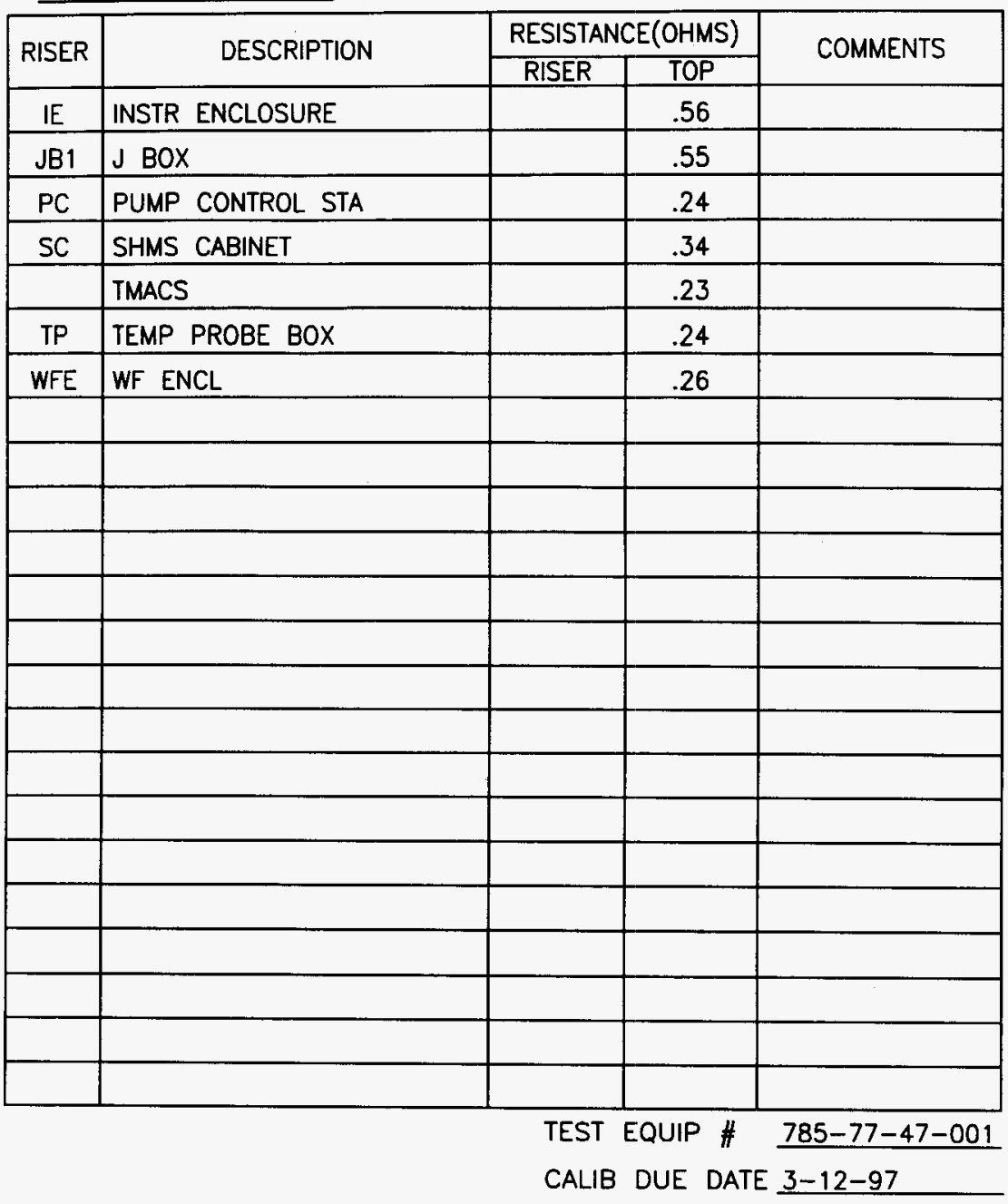


WHC-SD-WM-TR-034, REV. 0

\section{RISER RESISTANCE DATA SHEET}

TANK SX-106

\begin{tabular}{|c|c|c|c|c|}
\hline \multirow{2}{*}{ RISER } & \multirow{2}{*}{ DESCRIPTION } & \multicolumn{2}{|c|}{ RESISTANCE(OHMS) } & \multirow{2}{*}{ COMMENTS } \\
\hline & & RISER & TOP & \\
\hline 2 & 4" SPARE & .12 & .47 & \\
\hline 3 & 4" ENRAF & .12 & .15 & \\
\hline 6 & $12^{\prime \prime}$ OBSV PORT & .13 & .17 & \\
\hline 7 & 12" SPARE & .13 & 116.6 & \\
\hline (11) & 4" BREATHER FLTR & .21 & .21 & \\
\hline (14) & $4^{n}$ SPARE & 34.5 & 34.5 & U.G.R. \\
\hline (16) & 2.5" TEMP PROBE & .17 & .18 & \\
\hline $\mathrm{BB}$ & TEMP STAND & & .18 & \\
\hline EU & ENRAF UNISTRUT & & .13 & \\
\hline $\mathrm{PC}$ & PUMP CONTROL & & .53 & \\
\hline SC & SHMS CABINET & & .15 & \\
\hline $\mathrm{VH}$ & VALVE HANDLE & & 68.6 & \\
\hline WFE & WF ENCLOSURE & & .55 & \\
\hline & & & & \\
\hline & & & & \\
\hline
\end{tabular}

TEST EQUIP \# 785-77-47-001

CALIB DUE DATE 3-12-97 
WHC-SD-WM-TR-034, REV. 0

\section{RISER RESISTANCE DATA SHEET}

TANK SX-107
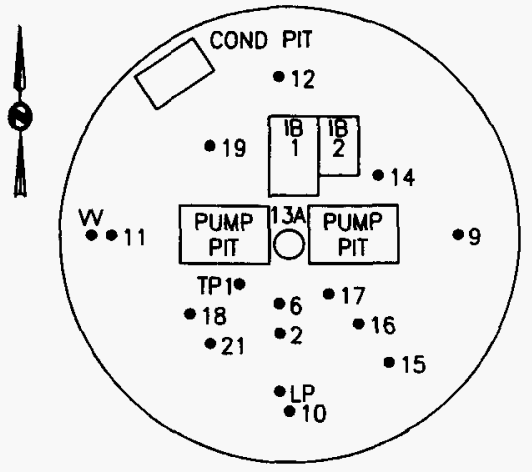

\begin{tabular}{|c|c|c|c|c|}
\hline \multirow{2}{*}{ RISER } & \multirow{2}{*}{ DESCRIPTION } & \multicolumn{2}{|c|}{ RESISTANCE(OHMS) } & \multirow{2}{*}{ COMMENTS } \\
\hline & & RISER & TOP & \\
\hline 2 & 4" REEL & .21 & OPEN & \\
\hline 6 & $12^{\prime \prime}$ OBSV PORT & .21 & 12.34 & \\
\hline (9) & $2.5^{\prime \prime}$ TEMP PROBE & 35 & 35 & U.G.R. \\
\hline (10) & 2.5" TEMP PROBE & .28 & .28 & \\
\hline (11) & $2.5^{n}$ TEMP PROBE & 3.46 & 3.46 & \\
\hline (12) & 2.5" TEMP PROBE & 3.99 & 3.99 & \\
\hline $13 \mathrm{~A}$ & 18" INLET FILTER & .21 & .21 & \\
\hline (14) & $2.5^{\prime \prime}$ TEMP PROBE & .30 & .30 & \\
\hline (15) & $2.5^{n}$ TEMP PROBE & 3.47 & 3.47 & \\
\hline (16) & 12" SPARE & 58 & 59 & U.G.R. \\
\hline (17) & 12" SPARE & 67 & 737 & U.G.R. \\
\hline (18) & 2.5" TEMP PROBE & 3.72 & 3.72 & \\
\hline (19) & $2.5^{n}$ TEMP PROBE & 75 & & U.G.R. \\
\hline (21) & 18" EXH PORT & .21 & .21 & \\
\hline$|\mathrm{B}|$ & INSTRUMENT BLDG & & .40 & \\
\hline \multicolumn{2}{|c|}{ CONTINUED NEXT SHEET } & \multicolumn{2}{|c|}{$\begin{array}{l}\text { TEST EQUIP \# } \\
\text { CALIB DUE DA }\end{array}$} & $\begin{array}{l}785-77-47-001 \\
3-12-97\end{array}$ \\
\hline
\end{tabular}




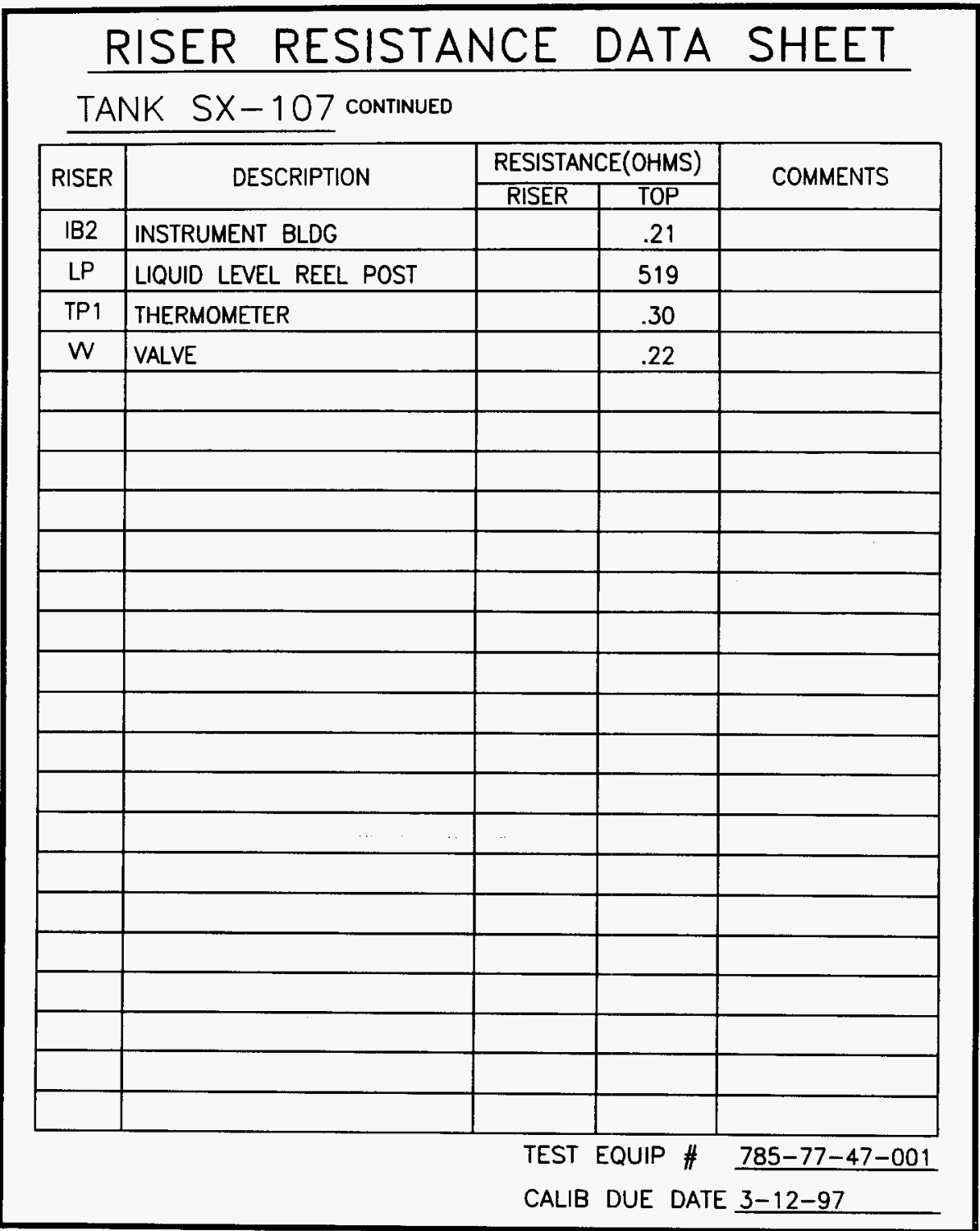




\section{RISER RESISTANCE DATA SHEET}

TANK SX-108
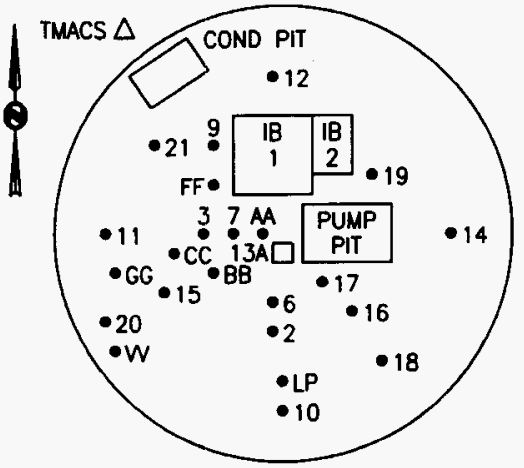

\begin{tabular}{|c|c|c|c|c|}
\hline \multirow{2}{*}{ RISER } & \multirow{2}{*}{ DESCRIPTION } & \multicolumn{2}{|c|}{ RESISTANCE(OHMS) } & \multirow{2}{*}{ COMMENTS } \\
\hline & & RISER & TOP & \\
\hline 2 & 4" REEL & 638 & 637 & U.G.R. \\
\hline 3 & 4" W/ THERMOMETER & .22 & .22 & \\
\hline 6 & 12" WEATHER & $*$ & 7.84 & NO ACCESS TO RISER \\
\hline 7 & $12^{n}$ SPARE & .22 & .22 & \\
\hline (9) & $2.5^{\prime \prime}$ TEMP PROBE & .25 & .25 & \\
\hline (10) & 2.5" TEMP PROBE & 21.9 & 21.9 & U.G.R. \\
\hline (11) & $2.5^{n}$ TEMP PROBE & 7.5 & 3.73 & U.G.R. \\
\hline (12) & $2.5^{\prime \prime}$ TEMP PROBE & 2.97 & 2.97 & \\
\hline $13 \mathrm{~A}$ & 18" INLET FILTER & .20 & .20 & \\
\hline (14) & 2.5" TEMP PROBE & 3.95 & 3.95 & \\
\hline (15) & $2.5^{\prime \prime}$ TEMP PROBE & 3.09 & 3.09 & \\
\hline 16 & $12^{\prime \prime}$ SPARE & 39.8 & 39.8 & U.G.R. \\
\hline (17) & 12" OBSV PORT & 44.5 & 44.5 & U.G.R. \\
\hline (18) & $2.5^{\prime \prime}$ TEMP PROBE & 2.53 & 2.53 & \\
\hline (19) & $2.5^{\prime \prime}$ TEMP PROBE & 22 & 22 & U.G.R. \\
\hline \multicolumn{2}{|c|}{ CONTINUED NEXT SHEET } & \multicolumn{2}{|c|}{ TEST EQUIP \# } & $\begin{array}{l}785-77-47-001 \\
3-12-97\end{array}$ \\
\hline
\end{tabular}




\section{RISER RESISTANCE DATA SHEET}

TANK SX -108 CONTINUED

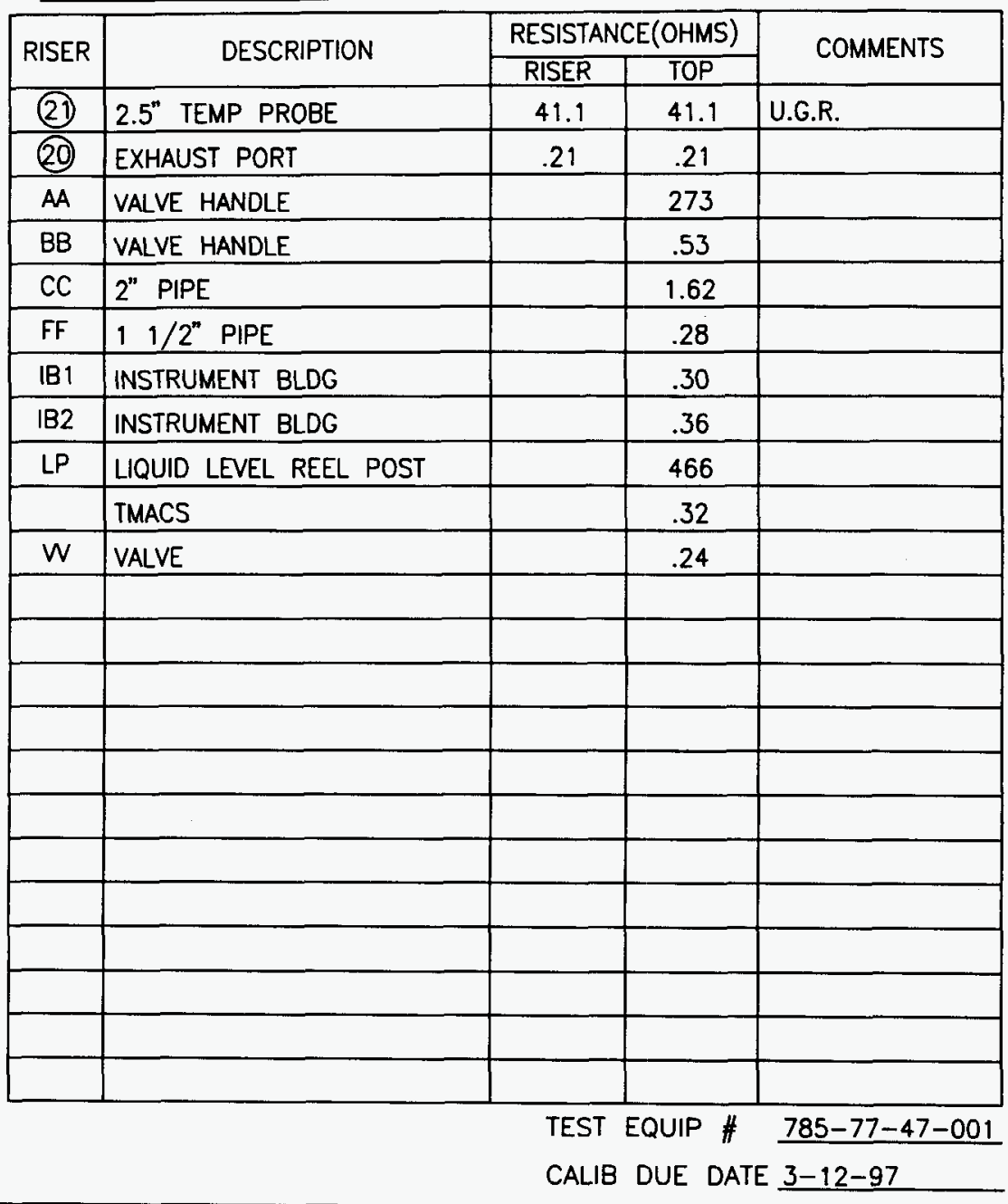




\section{RISER RESISTANCE DATA SHEET}

TANK SX-109
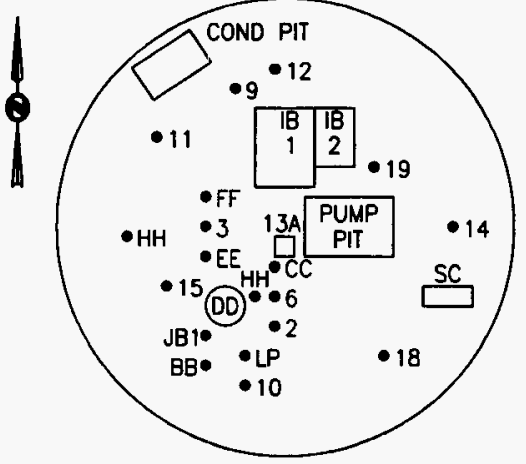

\begin{tabular}{|c|c|c|c|c|}
\hline \multirow{2}{*}{ RISER } & \multirow{2}{*}{ DESCRIPTION } & \multicolumn{2}{|c|}{ RESISTANCE(OHMS) } & \multirow{2}{*}{ COMMENTS } \\
\hline & & RISER & TOP & \\
\hline 2 & 4" REEL & .20 & OPEN & \\
\hline 3 & 4" SPARE W/THERMOMETER & .22 & .22 & \\
\hline 6 & 12" EXH PORT & .21 & .21 & \\
\hline (9) & $2.5^{\prime \prime}$ TEMP PROBE & .23 & .23 & \\
\hline (10) & $2.5^{n}$ TEMP PROBE & .34 & .34 & \\
\hline (11) & $2.5^{n}$ TEMP PROBE & .29 & .32 & \\
\hline (12) & $2.5^{\prime \prime}$ TEMP PROBE & .30 & .32 & \\
\hline $13 \mathrm{~A}$ & $18^{n}$ INLET FILTER & .22 & .22 & \\
\hline (14) & $2.5^{n}$ TEMP PROBE & .32 & .32 & \\
\hline (15) & $2.5^{\prime \prime}$ TEMP PROBE & .25 & .25 & \\
\hline (18) & 2.5" TEMP PROBE & .38 & .38 & \\
\hline (19) & 2.5" TEMP PROBE & .53 & .53 & \\
\hline BB & TEMP PROBE & & 35 & \\
\hline $\mathrm{CC}$ & VALVE HANDLE & & .33 & \\
\hline DD & $4^{\prime \prime}$ CULVERT & & .49 & \\
\hline \multicolumn{2}{|c|}{ CONTINUED NEXT SHEET } & \multicolumn{2}{|c|}{$\begin{array}{l}\text { TEST EQUIP \# } \\
\text { CALIB DUE DAT }\end{array}$} & $\begin{array}{l}85-77-47-001 \\
-12-97\end{array}$ \\
\hline
\end{tabular}




\section{RISER RESISTANCE DATA SHEET}

TANK SX-109 CONTINUED

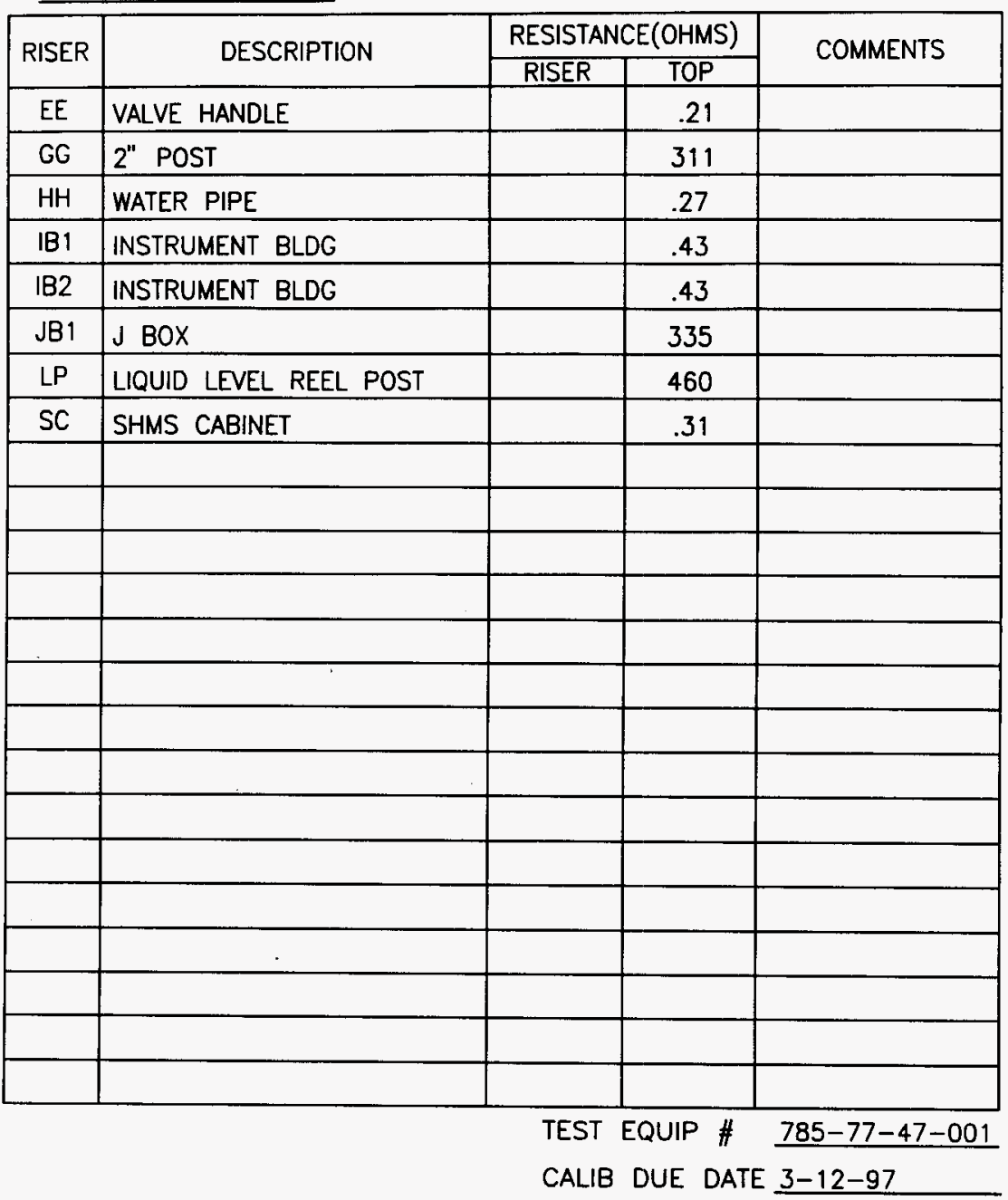




\section{RISER RESISTANCE DATA SHEET}

TANK SX-110
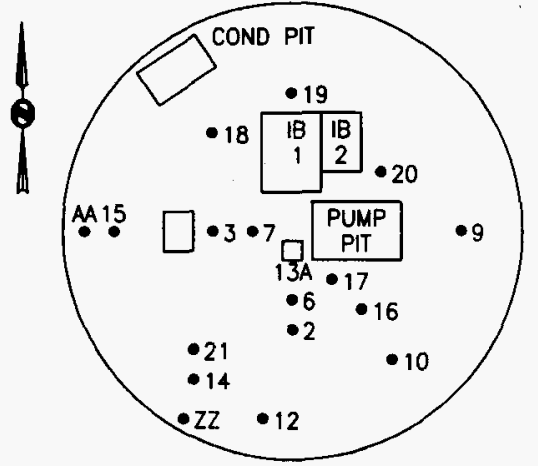

\begin{tabular}{|c|c|c|c|c|}
\hline \multirow{2}{*}{ RISER } & \multirow{2}{*}{ DESCRIPTION } & \multicolumn{2}{|c|}{ RESISTANCE(OHMS) } & \multirow{2}{*}{ COMMENTS } \\
\hline & & RISER & TOP & \\
\hline 2 & 4" REEL & .21 & OPEN & \\
\hline 3 & 4" TEMP PROBE & .22 & .23 & \\
\hline 6 & $12^{\prime \prime}$ & .22 & 1.55 & \\
\hline 7 & 12" DIP TUBE & .21 & 1.64 & \\
\hline (9) & $2.5^{n}$ TEMP PROBE & .23 & 8.5 & \\
\hline (10) & $2.5^{\prime \prime}$ TEMP PROBE & 9.8 & 3.2 & U.G.R. \\
\hline (12) & 2.5" TEMP PROBE & 240 & 230 & U.G.R. \\
\hline $13 A$ & 18" INLET FILTER & .22 & .22 & \\
\hline (14) & $2.5^{n}$ TEMP PROBE & 6.87 & 3.26 & G.T.W./U.G.R. \\
\hline (15) & 2.5" TEMP PROBE & 2.96 & 2.93 & \\
\hline 16 & 12" SPARE & .22 & .22 & \\
\hline 17 & 12" OBSV PORT & .23 & .38 & \\
\hline (18) & 2.5" TEMP PROBE & 27 & 2.81 & G.T.W./U.G.R. \\
\hline (19) & $2.5^{n}$ TEMP PROBE & 51 & 2.75 & G.T.W./U.G.R. \\
\hline (20) & 2.5" TEMP PROBE & 23 & 240 & U.G.R. \\
\hline
\end{tabular}

CONTINUED NEXT SHEET

TEST EQUIP \# 785-77-47-001

CALIB DUE DATE $3-12-97$ 


\section{RISER RESISTANCE DATA SHEET}

TANK SX-110 CONTINUED

\begin{tabular}{|c|c|c|c|c|}
\hline \multirow{2}{*}{ RISER } & \multirow{2}{*}{ DESCRIPTION } & \multicolumn{2}{|c|}{ RESISTANCE(OHMS) } & \multirow{2}{*}{ COMMENTS } \\
\hline & & RISER & TOP & \\
\hline (21) & EXHAUST PORT & .22 & .22 & \\
\hline AA & VALVE & & .26 & \\
\hline IB1 & INSTRUMENT BLDG & & .53 & \\
\hline IB2 & INSTRUMENT BLDG & & .53 & \\
\hline$Z Z$ & $1.5^{n} \mathrm{PIPE}$ & & 5.74 & \\
\hline & & & & \\
\hline & & & & \\
\hline & & & & \\
\hline & & & & \\
\hline & & & & \\
\hline & & & & \\
\hline & & & & \\
\hline & & & & \\
\hline & & & & \\
\hline & & & & \\
\hline & & & & \\
\hline & & & & \\
\hline & & & & \\
\hline & & & & \\
\hline & & & & \\
\hline & & & & \\
\hline & & & & \\
\hline & & & & \\
\hline
\end{tabular}

TEST EQUIP \# 785-77-47-001

CALIB DUE DATE 3-12-97 
WHC-SD-WM-TR-034, REV. 0

\section{RISER RESISTANCE DATA SHEET}

\begin{tabular}{|c|c|c|c|}
\hline \multirow{2}{*}{ RISER } & \multirow{2}{*}{ DESCRIPTION } & \multicolumn{2}{|c|}{ RESISTANCE(OHMS) } \\
\hline & & RISER & \multirow{2}{*}{$\begin{array}{l}\text { TOP } \\
\text { OPEN }\end{array}$} \\
\hline 2 & 4" REEL & $1.66 \mathrm{~K}$ & \\
\hline 3 & 4" WITH THERMOMETER & .21 & 5.37 \\
\hline 6 & 12" SPARE & .60 & .60 \\
\hline 7 & $12^{\prime \prime}$ SPARE & .22 & .34 \\
\hline (9) & 2.5" TEMP PROBE & 2.07 & 2.07 \\
\hline (10) & $2.5^{\prime \prime}$ TEMP PROBE & 2.26 & 2.64 \\
\hline (11) & $2.5^{\prime \prime}$ TEMP PROBE & 2.26 & 2.22 \\
\hline (12) & 2.5" TEMP PROBE & 1.33 & 1.33 \\
\hline $13 \mathrm{~A}$ & $18^{\prime \prime}$ INLET FILTER & .23 & .23 \\
\hline (14) & 2.5" TEMP PROBE & 7.23 & 7.23 \\
\hline (15) & 2.5" TEMP PROBE & 2.27 & 2.27 \\
\hline 16 & $12^{\prime \prime}$ OBSV PORT & .21 & 61 \\
\hline 17 & $12^{\prime \prime}$ SPARE & .21 & .21 \\
\hline (18) & 2.5" TEMP PROBE & 2.09 & 2.09 \\
\hline (19) & $2.5^{\prime \prime}$ TEMP PROBE & .58 & .58 \\
\hline 20 & 12" EXH PORT & .22 & .22 \\
\hline \multicolumn{2}{|c|}{ CONTINUED NEXT SHEET } & \multicolumn{2}{|c|}{$\begin{array}{l}\text { TEST EQUIP \# } \\
\text { CALIB DUE DAT }\end{array}$} \\
\hline
\end{tabular}




\section{RISER RESISTANCE DATA SHEET}

\section{TANK SX-111 CONTINUED}

\begin{tabular}{|c|c|c|c|c|}
\hline \multirow{2}{*}{ RISER } & \multirow{2}{*}{ DESCRIPTION } & \multicolumn{2}{|c|}{ RESISTANCE(OHMS) } & \multirow{2}{*}{ COMMENTS } \\
\hline & & RISER & TOP & \\
\hline BB & VALVE HANDLE & & .23 & \\
\hline $\mathrm{DD}$ & VALVE & & .22 & \\
\hline ENC & INSTR ENCLOSURE & & .24 & \\
\hline 181 & INSTRUMENT BLDG & & .24 & \\
\hline IB2 & INSTRUMENT BLDG & & .24 & \\
\hline LP & LIQUID LEVEL REEL POST & & 404 & \\
\hline \multirow[t]{2}{*}{ PC } & PUMP CONTROL & & .23 & \\
\hline & TMACS & & .27 & \\
\hline & & & & \\
\hline & & & & \\
\hline & & & & \\
\hline & & & & \\
\hline & & & & \\
\hline & & & & \\
\hline & & & & \\
\hline & & & & \\
\hline & & & & \\
\hline & & & & \\
\hline & & & & \\
\hline & & & & \\
\hline & & & & \\
\hline & & & & \\
\hline & & & & \\
\hline & & $\begin{array}{l}\text { TES } \\
\text { CALI }\end{array}$ & $\begin{array}{l}\text { UIP \# } \\
\text { UE DA }\end{array}$ & $\begin{array}{l}85-77-47- \\
-12-97\end{array}$ \\
\hline
\end{tabular}




\section{RISER RESISTANCE DATA SHEET}

TANK SX-112

\begin{tabular}{|c|c|c|c|c|}
\hline \multirow{2}{*}{ RISER } & \multirow{2}{*}{ DESCRIPTION } & \multicolumn{2}{|c|}{ RESISTANCE(OHMS) } & \multirow{2}{*}{ COMMENTS } \\
\hline & & RISER & TOP & \\
\hline 2 & 4" REEL & .21 & OPEN & \\
\hline 3 & 4" WITH THERMOMETER & & .22 & RISER ENCASED \\
\hline 6 & 12" OBSV PORT & .22 & $1.91 \mathrm{~K}$ & \\
\hline (9) & $2.5^{n}$ TEMP PROBE & .49 & .49 & \\
\hline (10) & 2.5" TEMP PROBE & .51 & .51 & \\
\hline (11) & 2.5" TEMP PROBE & 2.02 & 2.02 & \\
\hline (12) & $2.5^{n}$ TEMP PROBE & 1.66 & 1.66 & \\
\hline $13 \mathrm{~A}$ & 18" INLET FILTER & .23 & .23 & \\
\hline (14) & 2.5" TEMP PROBE & 1.36 & 1.36 & \\
\hline (15) & 2.5" TEMP PROBE & .29 & .29 & \\
\hline (18) & $2.5^{n}$ TEMP PROBE & .81 & .81 & \\
\hline (19) & $2.5^{\prime \prime}$ TEMP PROBE & 4.8 & 4.79 & \\
\hline (20) & $12^{\prime \prime}$ EXH PORT & .21 & .21 & \\
\hline AA & VALVE & & .31 & \\
\hline $\mathrm{CC}$ & FLANGE & & 293 & \\
\hline \multicolumn{2}{|c|}{ CONTINUED NEXT SHEET } & \multicolumn{2}{|c|}{$\begin{array}{l}\text { TEST EQUIP \# } \\
\text { CALIB DUE DAT }\end{array}$} & $\begin{array}{l}785-77-47-001 \\
3-12-97\end{array}$ \\
\hline
\end{tabular}

CONTINUED NEXT SHEET

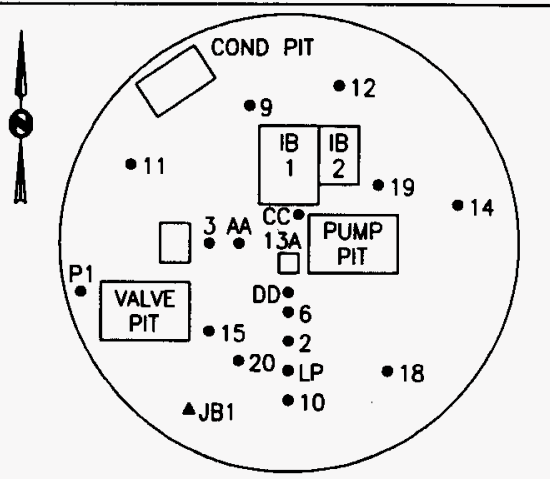




\section{RISER RESISTANCE DATA SHEET}

TANK SX-112 CONTINUED

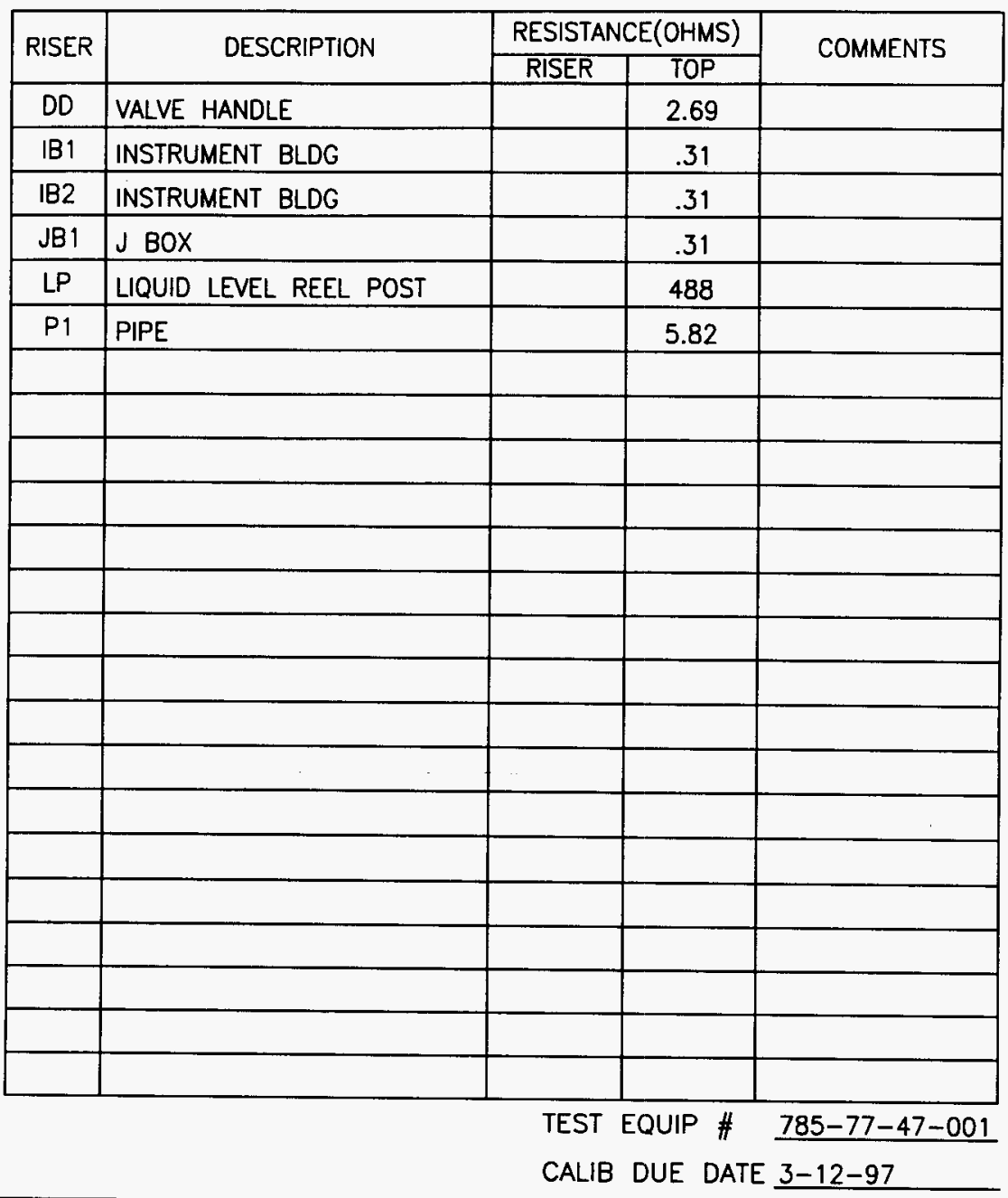




\section{RISER RESISTANCE DATA SHEET}

TANK SX-113

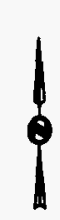

\begin{tabular}{|c|c|c|c|c|}
\hline \multirow{2}{*}{ RISER } & \multirow{2}{*}{ DESCRIPTION } & \multicolumn{2}{|c|}{ RESISTANCE(OHMS) } & \multirow{2}{*}{ COMMENTS } \\
\hline & & RISER & TOP & \\
\hline 2 & 4" REEL & .22 & OPEN & \\
\hline 3 & $\begin{array}{l}\text { 4" TEMP PROBE WITH } \\
\text { THERMOMETER }\end{array}$ & .23 & .23 & \\
\hline 4 & 4" SPARE/BOX & .22 & .22 & \\
\hline 6 & $12^{n}$ BREATHER FLTR & .24 & .22 & \\
\hline 7 & 12" OBSV PORT & .22 & 1.83 & \\
\hline $\mathrm{CC}$ & $11 / 2^{n}$ PIPE/BOX & & 8.16 & \\
\hline JB1 & $\mathrm{J} B O X$ & & 562 & \\
\hline LP & LIQUID LEVEL REEL POST & & 553 & \\
\hline & & & & \\
\hline & & & & \\
\hline & & & & \\
\hline & & & & \\
\hline & & & & \\
\hline & & & & \\
\hline
\end{tabular}

TEST EQUIP \#

CALIB DUE DATE 3-12-97 


\section{RISER RESISTANCE DATA SHEET}

TANK SX-114

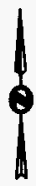

COND PIT

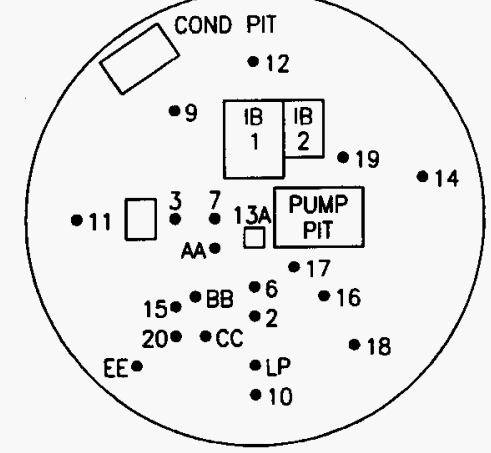

\begin{tabular}{|c|c|c|c|c|}
\hline \multirow{2}{*}{ RISER } & \multirow{2}{*}{ DESCRIPTION } & \multicolumn{2}{|c|}{ RESISTANCE(OHMS) } & \multirow{2}{*}{ COMMENTS } \\
\hline & & RISER & TOP & \\
\hline 2 & 4" REEL & .22 & OPEN & \\
\hline 3 & 4" THERMOMETER & .22 & .22 & \\
\hline 6 & 12" SPRINKLER & .23 & 909 & \\
\hline 7 & 12" SPARE & * & 2.06 & *NO ACCESS TO RISER \\
\hline (9) & $2.5^{\prime \prime}$ TEMP PROBE & .26 & .26 & \\
\hline (10) & $2.5^{n}$ TEMP PROBE & .27 & .27 & \\
\hline (11) & $2.5^{\prime \prime}$ TEMP PROBE & .27 & .27 & \\
\hline (12) & 2.5" TEMP PROBE & .32 & .32 & \\
\hline $13 \mathrm{~A}$ & 18" INLET FILTER & .21 & .21 & \\
\hline (14) & $2.5^{n}$ TEMP PROBE & .29 & .29 & \\
\hline (15) & $2.5^{n}$ TEMP PROBE & .27 & .27 & \\
\hline (16) & 12" PLUG & .23 & .32 & \\
\hline (17) & $23^{\prime \prime}$ OBSV PORT & 30 & 218 & U.G.R. \\
\hline (18) & $2.5^{\prime \prime}$ TEMP PROBE & .32 & .32 & \\
\hline (19) & 2.5" TEMP PROBE & .26 & .25 & \\
\hline \multirow{2}{*}{\multicolumn{2}{|c|}{ CONTINUED NEXT SHEET }} & \multicolumn{2}{|c|}{ TEST EQUIP } & $785-77-47-001$ \\
\hline & & \multicolumn{3}{|c|}{ CALIB DUE DATE $3-12-97$} \\
\hline
\end{tabular}




\section{RISER RESISTANCE DATA SHEET}

TANK SX-114 CONTINUED

\begin{tabular}{|c|c|c|c|c|}
\hline \multirow{2}{*}{ RISER } & \multirow{2}{*}{ DESCRIPTION } & \multicolumn{2}{|c|}{ RESISTANCE(OHMS) } & \multirow{2}{*}{ COMMENTS } \\
\hline & & RISER & TOP & \\
\hline 20 & $24^{\prime \prime}$ EXH PORT & .22 & .22 & \\
\hline AA & VALLE HANDLE & & .32 & \\
\hline $\mathrm{BB}$ & $11 / 2^{n}$ PIPE & & 14.12 & \\
\hline $\mathrm{CC}$ & TEMP PROBE & & .22 & \\
\hline$E E$ & VALVE & & .23 & \\
\hline LP & LIQUID LEVEL REEL POST & & 429 & \\
\hline IB1 & INSTRUMENT BLDG & & .25 & \\
\hline IB2 & INSTRUMENT BLDG & & .25 & \\
\hline & & & & \\
\hline & & & & \\
\hline & & & & \\
\hline & & & & \\
\hline & & & & \\
\hline & & & & \\
\hline & & & & \\
\hline & & & & \\
\hline & & & & \\
\hline & & & & \\
\hline & & & & \\
\hline & & & & \\
\hline & & & & \\
\hline & & & & \\
\hline & & & & \\
\hline & & TEST & QUIP \# & $785-77-47-001$ \\
\hline & & CALI & OUE DAT & $3-12-97$ \\
\hline
\end{tabular}


WHC-SD-WM-TR-034, REV. 0

\section{RISER RESISTANCE DATA SHEET}

TANK SX-115

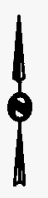

COND PIT

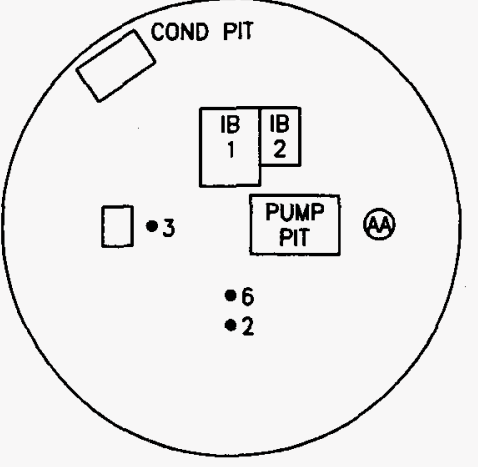

\begin{tabular}{|c|c|c|c|c|}
\hline \multirow{2}{*}{ RISER } & \multirow{2}{*}{ DESCRIPTION } & \multicolumn{2}{|c|}{ RESISTANCE(OHMS) } & \multirow{2}{*}{ COMMENTS } \\
\hline & & RISER & TOP & \\
\hline 2 & 4" REEL & .22 & OPEN & \\
\hline 3 & 4" BREATHER FLTR & .21 & .63 & \\
\hline 6 & $12^{n}$ OBSV PORT & .27 & .27 & \\
\hline$A A$ & VALVE & & .20 & \\
\hline IB1 & INSTRUMENT BLDG & & .27 & \\
\hline IB2 & INSTRUMENT BLDG & & .27 & \\
\hline & & & & \\
\hline & & & & \\
\hline & & & & \\
\hline & & & & \\
\hline & & & & \\
\hline & & & & \\
\hline & & & & \\
\hline & & & & \\
\hline & & TES & XUIP \# & $785-77-47-001$ \\
\hline & & CAL & UE DA & $3-12-97$ \\
\hline
\end{tabular}




\section{RISER RESISTANCE DATA SHEET}

\begin{tabular}{|c|c|c|c|c|}
\hline \multirow{2}{*}{ RISER } & \multirow{2}{*}{ DESCRIPTION } & \multicolumn{2}{|c|}{ RESISTANCE(OHMS) } & \multirow{2}{*}{ COMMENTS } \\
\hline & & RISER & TOP & \\
\hline 1 & 4" ENRAF & 1.21 & 1.57 & \\
\hline 2 & 12" OBSV PORT & 1.21 & 1.22 & \\
\hline 7 & $\begin{array}{l}12 " \text { BREATHER } \\
\text { MANUAL TAPE }\end{array}$ & 1.22 & 1.23 & \\
\hline 8 & 4" TEMP PROBE & 1.22 & 1.24 & \\
\hline EU & ENRAF UNISTRUT & & 1.64 & \\
\hline WR & $\begin{array}{l}\text { WELDING RCPT } \\
\text { MTG HARDWARE }\end{array}$ & & 40.1 & \\
\hline EE & ELEC ENCLOSURE & & 4.68 & \\
\hline RS & RECEPTACLE STAND & & 4.75 & \\
\hline WF & WF CABINET & & 1.35 & \\
\hline PCS & PUMP CONTROL STA & & 1.23 & \\
\hline$T M$ & TMACS & & 1.24 & \\
\hline & & $\begin{array}{l}\text { TES } \\
\text { CAL }\end{array}$ & $\begin{array}{l}\text { QUIP \# } \\
\text { OUE DA }\end{array}$ & $\begin{array}{l}785-77-47-001 \\
3-12-97\end{array}$ \\
\hline
\end{tabular}




\section{RISER RESISTANCE DATA SHEET}

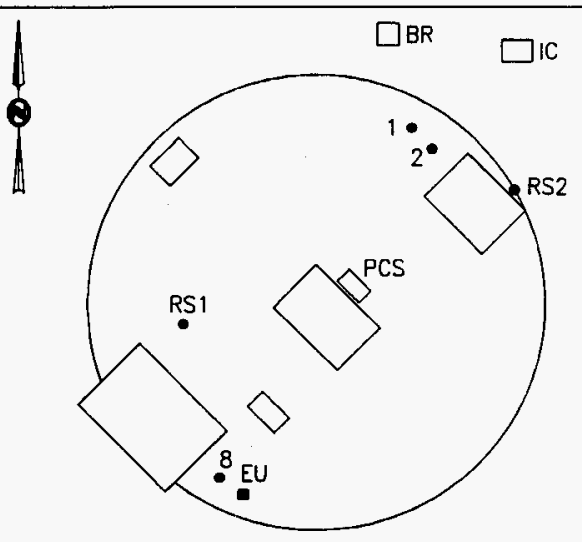

TANK T-102

\begin{tabular}{|l|l|c|c|c|}
\hline \multirow{2}{*}{ RISER } & \multirow{2}{*}{ DESCRIPTION } & \multicolumn{2}{c|}{ RESISTANCE(OHMS) } & \multirow{2}{*}{ COMMENTS } \\
\cline { 3 - 4 } & & RISER & TOP & \\
\hline \hline 1 & $4 "$ BREATHER & 1.21 & 3.68 & \\
\hline 2 & $12^{\prime \prime}$ OBSV PORT & 1.22 & 1.22 & \\
\hline 8 & $4 "$ ENRAF & 1.22 & 1.22 & \\
\hline BR & BURIED RAIL & & 13.87 & \\
\hline IC & INSTR CABINET & & 4.70 & \\
\hline RS2 & RECEPTACLE STAND & & 4.70 & \\
\hline PCS & PUMP CONTROL STA & & 143.9 & \\
\hline RS1 & RECEPTACLE STAND & & 4.7 & \\
\hline EU & ENRAF UNISTRUT & & 1.22 & \\
\hline & & & & \\
\hline
\end{tabular}

TEST EQUIP \# 785-77-47-001

CALIB DUE DATE 3-12-97 


\section{RISER RESISTANCE DATA SHEET}

TANK T-103

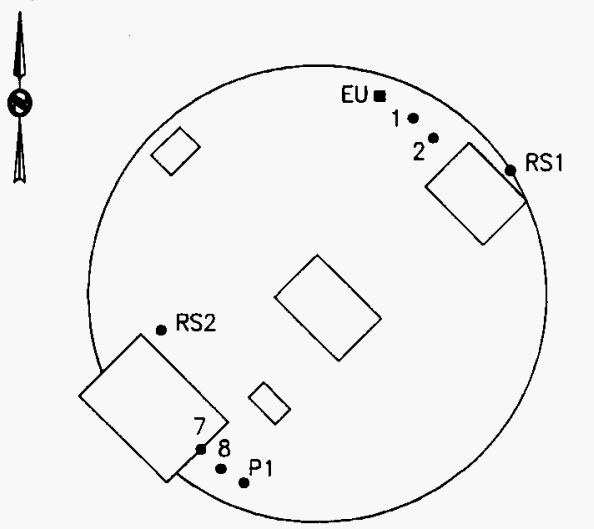

\begin{tabular}{|c|c|c|c|c|}
\hline \multirow{2}{*}{ RISER } & \multirow{2}{*}{ DESCRIPTION } & \multicolumn{2}{|c|}{ RESISTANCE(OHMS) } & \multirow{2}{*}{ COMMENTS } \\
\hline & & RISER & TOP & \\
\hline 1 & 4" ENRAF & .35 & .70 & \\
\hline 2 & 12" OBSV PORT & .35 & .35 & \\
\hline 7 & 12" BREATHER & .41 & .50 & \\
\hline 8 & 4" TEMP PROBE & .35 & .33 & \\
\hline EU & ENRAF UNISTRUT & & .69 & \\
\hline RS1 & RECEPTACLE STAND & & 10.68 & \\
\hline RS2 & RECEPTACLE STAND & & 10.60 & \\
\hline P1 & 3/4" PIPE & & 4.56 & \\
\hline & & & & \\
\hline & & & & \\
\hline
\end{tabular}

TEST EQUIP \# 785-77-47-001 CALIB DUE DATE 3-12-97 


\section{RISER RESISTANCE DATA SHEET}

TANK T-104
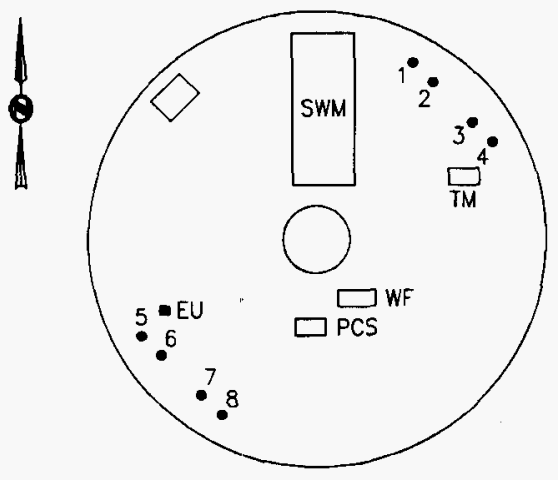

\begin{tabular}{|c|c|c|c|c|}
\hline \multirow{2}{*}{ RISER } & \multirow{2}{*}{ DESCRIPTION } & \multicolumn{2}{|c|}{ RESISTANCE(OHMS) } & \multirow{2}{*}{ COMMENTS } \\
\hline & & RISER & TOP & \\
\hline 1 & 4" LOW BM & .86 & & \\
\hline 2 & $12 "$ DIP TUBE & .85 & .85 & \\
\hline 3 & 12" TEMP VPR PROBE & .89 & 1.39 & \\
\hline 4 & 4" TEMP PROBE & .86 & & $\begin{array}{l}\text { BONDING JUMPER } \\
\text { TO SWM }\end{array}$ \\
\hline 5 & 4" ENRAF & .89 & .92 & \\
\hline 6 & 12" OBSV PORT & .90 & 1.00 & \\
\hline 7 & 12" CONC PLUG & .90 & 12.37 & \\
\hline 8 & 4" BREATHER & 1.00 & 76.2 & \\
\hline SWM & $\begin{array}{l}\text { SALTWELL MONITORING } \\
\text { \& EQUUPMENT } \\
\end{array}$ & & .86 & \\
\hline TM & TMACS & & .91 & \\
\hline EU & ENRAF UNISTRUT & & .96 & \\
\hline WF & WF CABINET & & 1.27 & \\
\hline PCS & PUMP CONTROL STA & & .93 & \\
\hline
\end{tabular}

TEST EQUIP \# 785-77-47-001

CALIB DUE DATE 3-12-97 


\section{RISER RESISTANCE DATA SHEET}

TANK T-105

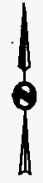

\begin{tabular}{|c|c|c|c|c|}
\hline \multirow{2}{*}{ RISER } & \multirow{2}{*}{ DESCRIPTION } & \multicolumn{2}{|c|}{ RESISTANCE(OHMS) } & \multirow{2}{*}{ COMMENTS } \\
\hline & & RISER & $\mathrm{TOP}$ & \\
\hline 1 & 4" ENRAF & 1.10 & 1.02 & \\
\hline 2 & 12" OBSV PORT & 1.10 & 17.76 & \\
\hline 3 & $12^{\prime \prime}$ FLG W/ LEAD & 1.11 & 1.13 & \\
\hline 4 & 4" TEMP (CUT) & 1.11 & 1.12 & \\
\hline 5 & 4" BREATHER & 1.11 & 1.13 & \\
\hline 6 & 12" RECIRC DIP TUBE & 1.10 & 1.11 & \\
\hline 7 & 12" FLG W/ LEAD & & & TEMP COVER \\
\hline 8 & 4" SPARE & 1.10 & 4.10 & \\
\hline EU & ENRAF UNISTRUT & & 1.07 & \\
\hline
\end{tabular}

TEST EQUIP \# 785-77-47-001

CALIB DUE DATE 3-12-97 


\section{RISER RESISTANCE DATA SHEET}

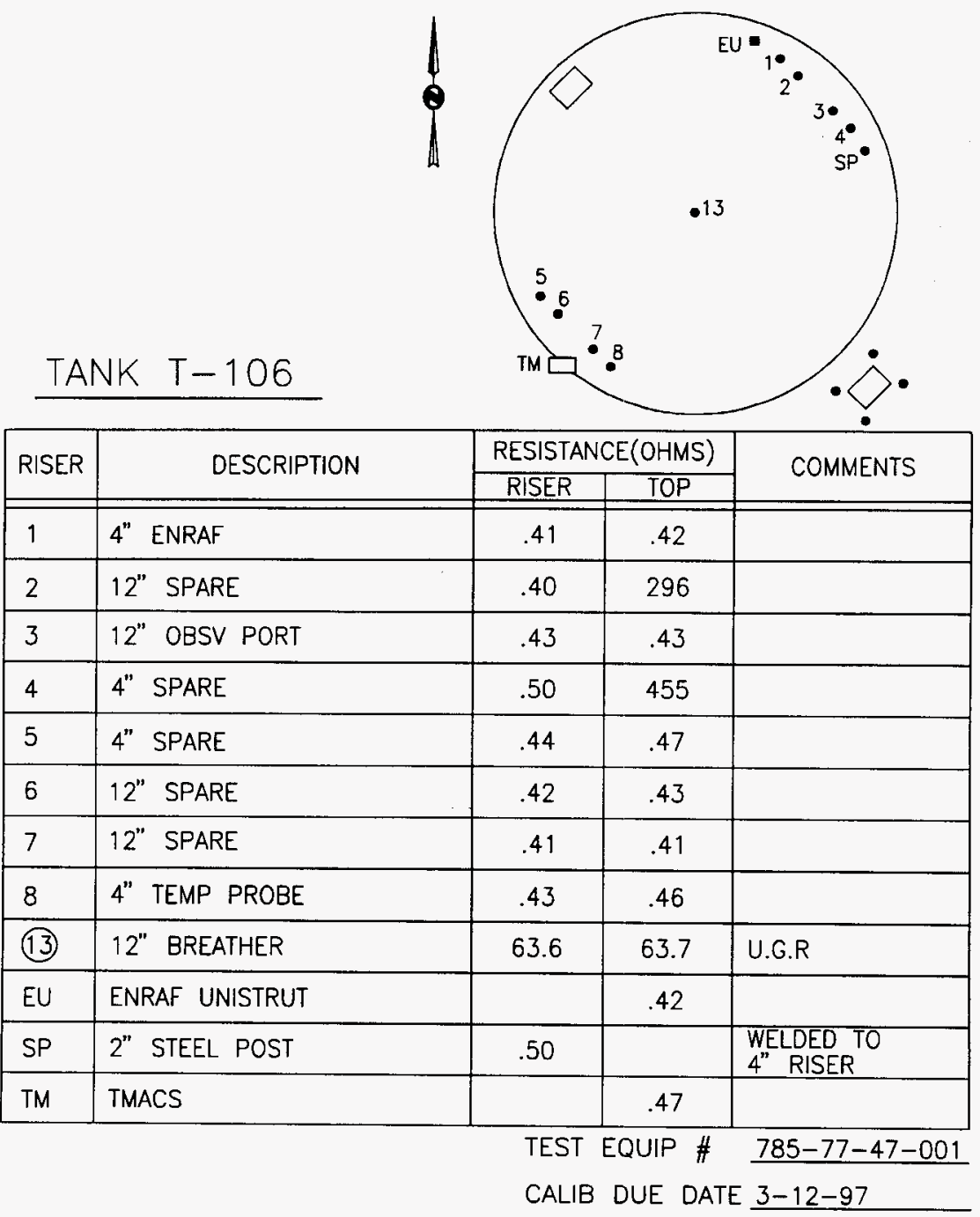




\section{RISER RESISTANCE DATA SHEET}

\begin{tabular}{|l|l|c|c|c|}
\hline \multicolumn{3}{|c|}{} & & \\
\hline
\end{tabular}




\section{RISER RESISTANCE DATA SHEET}

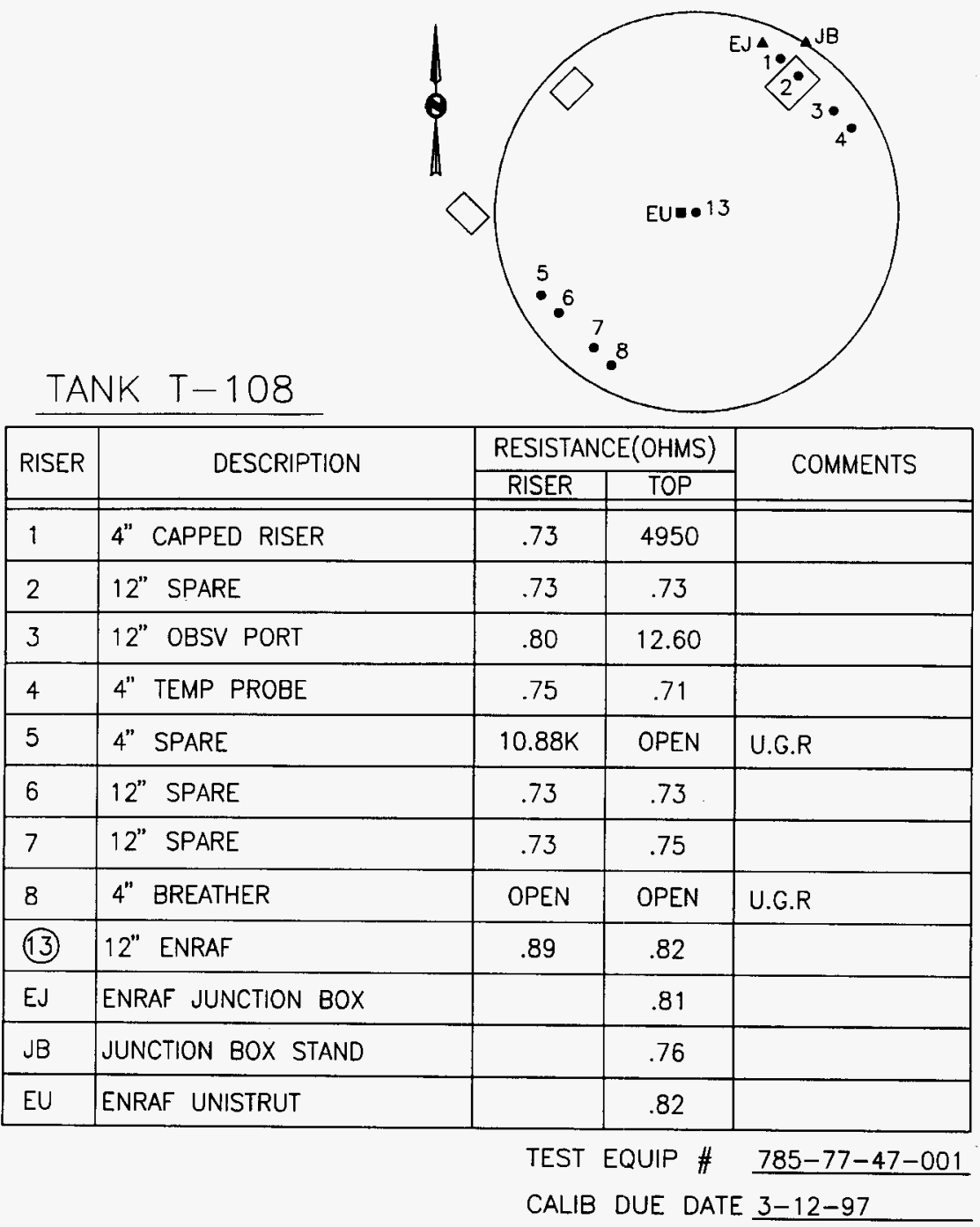




\section{RISER RESISTANCE DATA SHEET}

TANK T-109
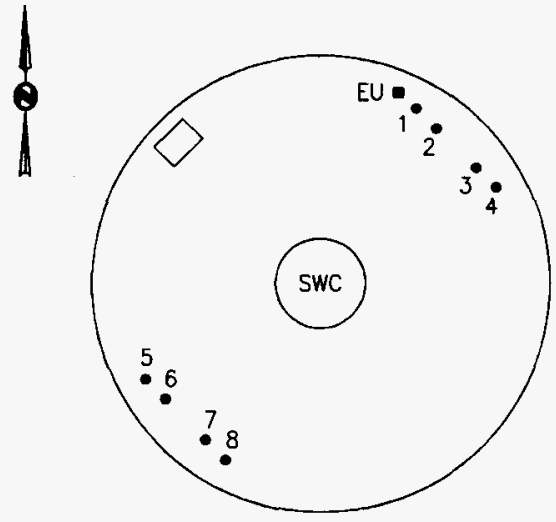

\begin{tabular}{|l|l|c|c|c|}
\hline \multirow{2}{*}{ RISER } & \multirow{2}{*}{ DESCRIPTION } & \multicolumn{2}{|c|}{ RESISTANCE(OHMS) } & \multirow{2}{*}{ COMMENTS } \\
\cline { 3 - 4 } & & RISER & TOP & \multirow{2}{*}{ COP } \\
\hline \hline 1 & $4 "$ ENRAF & .82 & .86 & \\
\hline 2 & $12 "$ DIP TUBES & .80 & .81 & \\
\hline 3 & $12 "$ BREATHER & .80 & .93 & \\
\hline 4 & $4 "$ SPARE & .82 & .87 & \\
\hline 5 & $4 "$ SPARE & .81 & .81 & \\
\hline 6 & $12 "$ SPARE & .83 & .83 & \\
\hline 7 & $12 "$ SPARE & .83 & .84 & \\
\hline 8 & $4 "$ TEMP PROBE & .80 & .81 & \\
\hline EU & ENRAF UNISTRUT & & .86 & \\
\hline SWC & SALTWELL COVER & & 24.4 & \\
\hline
\end{tabular}

TEST EQUIP \# 785-77-47-001

CALIB DUE DATE 3-12-97 


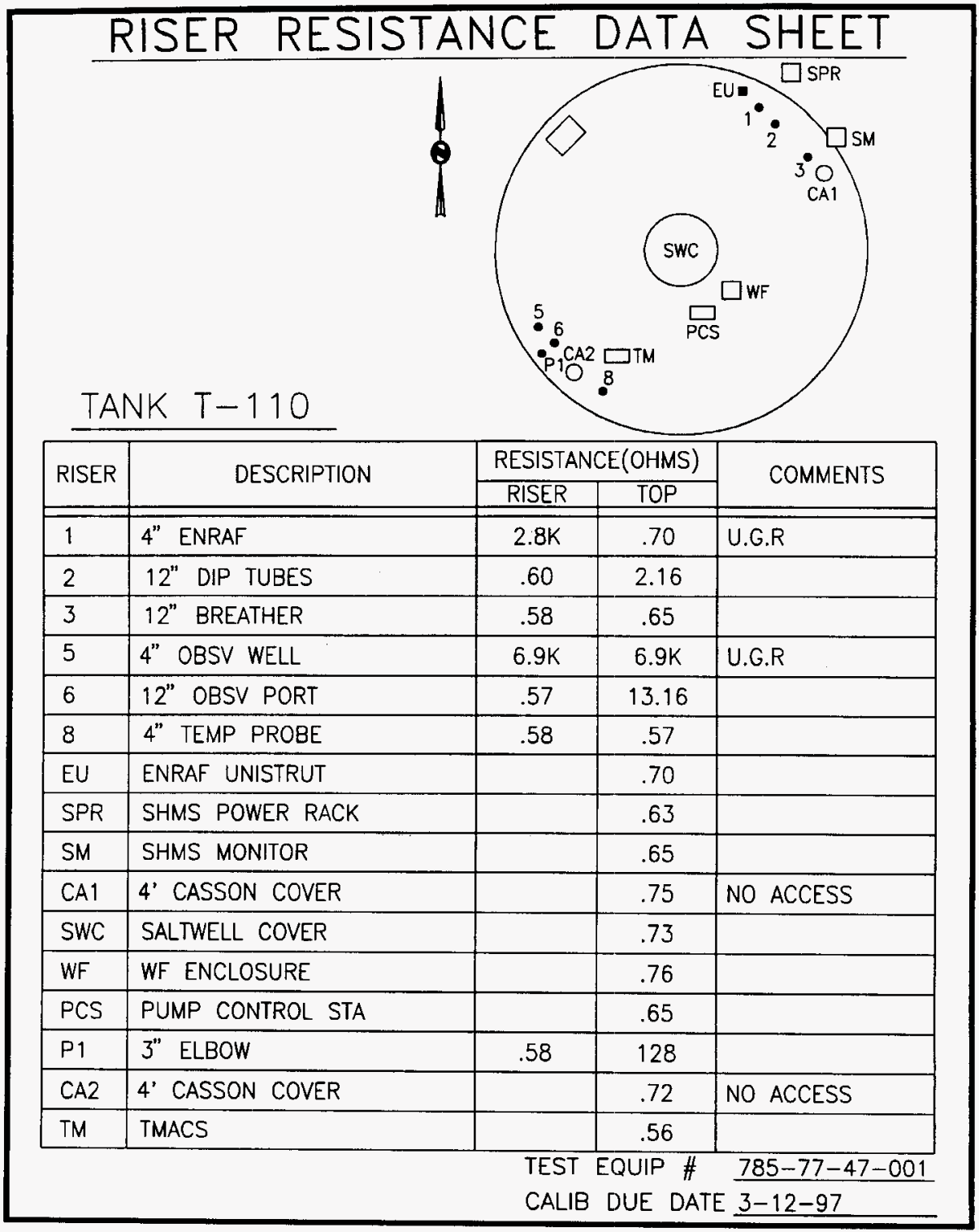




\section{RISER RESISTANCE DATA SHEET}

\begin{tabular}{|c|c|c|c|c|}
\hline \multirow{2}{*}{ RISER } & \multirow{2}{*}{ DESCRIPTION } & \multicolumn{2}{|c|}{ RESISTANCE(OHMS) } & \multirow{2}{*}{ COMMENTS } \\
\hline & & RISER & TOP & \\
\hline 2 & 12" SPARE & .50 & .52 & \\
\hline 3 & $\begin{array}{l}12^{\prime \prime} \text { OBSV PORT/ } \\
\text { BREATHER }\end{array}$ & .51 & .51 & \\
\hline 4 & 4" ENRAF & 1690 & $4.6 *$ & *FIRST SPOOL U.G.R. \\
\hline 5 & 4" TEMP PROBE & .69 & .32 & G.T.W. \\
\hline 6 & 12" SPARE & .51 & .51 & \\
\hline 7 & 12" LOW & .53 & .54 & \\
\hline $\mathrm{JB}$ & JUNCTION BOXES & & .45 & \\
\hline P1 & 3" ELBOW & .30 & .30 & \\
\hline EU & ENRAF UNISTRUT & & .49 & \\
\hline SWC & SALTWELL COVER & & .46 & \\
\hline PCS & PUMP CONTROL STA & & .40 & \\
\hline WF & WF CABINET & & .59 & \\
\hline$R C$ & RECEPTACLE & & 18.95 & \\
\hline TM & TMACS & & .28 & \\
\hline
\end{tabular}

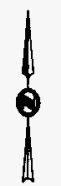

$\mathrm{RC}$
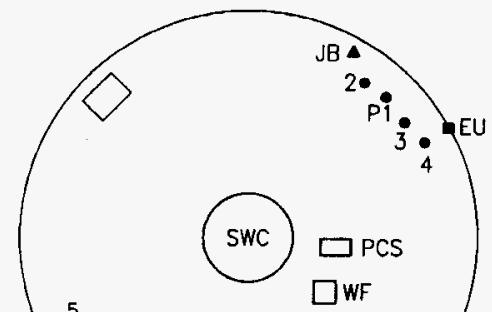

$\square$ PCS

$\square$ WF

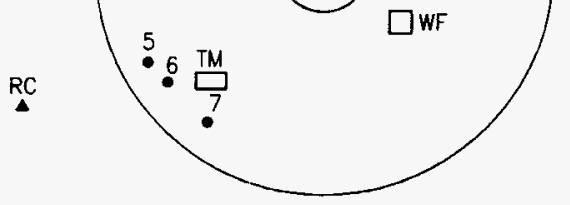

TEST EQUIP \# 785-77-47-001

CALIB DUE DATE 3-12-97 


\section{RISER RESISTANCE DATA SHEET}

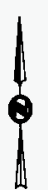

TANK T-112

\begin{tabular}{|c|c|c|c|c|}
\hline \multirow{2}{*}{ RISER } & \multirow{2}{*}{ DESCRIPTION } & \multicolumn{2}{|c|}{ RESISTANCE(OHMS) } & \multirow{2}{*}{ COMMENTS } \\
\hline & & RISER & TOP & \\
\hline 1 & 4" CAPPED & OPEN & OPEN & U.G.R. \\
\hline 2 & $12 "$ OBSV PORT & .48 & .48 & \\
\hline 3 & 12" RECIRC DIP TUBES & .47 & .47 & \\
\hline 4 & 4" SPARE & 732 & 732 & U.G.R \\
\hline 5 & 4" SPARE & .47 & .48 & \\
\hline 6 & $12^{\prime \prime}$ BREATHER & .47 & 14.86 & \\
\hline 7 & 12" PLUGGED & .47 & .63 & \\
\hline 8 & 4" TEMP PROBE & .46 & .46 & \\
\hline (13) & 12" ENRAF & .70 & & \\
\hline EJ & ENRAF JUNCTION BOX & & .66 & \\
\hline P1 & 3" ELBOW & .43 & 93.8 & \\
\hline $\mathrm{P} 2$ & 2" PIPE & .49 & & \\
\hline EU & ENRAF UNISTRUT & .68 & & \\
\hline $\mathrm{CS}$ & CONTROL STAND & & 45.1 & \\
\hline TM & TMACS & & .43 & \\
\hline
\end{tabular}

TEST EQUIP \# 785-77-47-001

CALIB DUE DATE 3-12-97 


\section{RISER RESISTANCE DATA SHEET}

TANK T-201

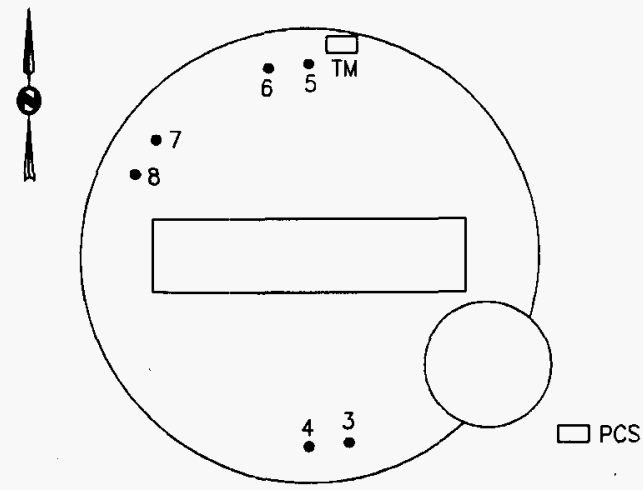

\begin{tabular}{|l|l|c|c|l|}
\hline \multirow{2}{*}{ RISER } & \multirow{2}{*}{ DESCRIPTION } & \multicolumn{2}{c|}{ RESISTANCE(OHMS) } & \multirow{2}{*}{ COMMENTS } \\
\cline { 3 - 4 } & & RISER & TOP & \\
\hline 3 & $12 "$ SPARE & 32.8 & 87.0 & U.G.R \\
\hline 4 & $4 "$ LIQ LEVEL REEL & 54.5 & 7900 & U.G.R \\
\hline 5 & $4 "$ TEMP PROBE & .93 & .89 & G.T.W. \\
\hline 6 & $12 "$ BREATHER & 34.4 & 34.4 & U.G.R \\
\hline 7 & $12 "$ OBSV PORT & 32 & 747 & U.G.R \\
\hline 8 & $4 "$ SPARE & 43.5 & 494 & U.G.R \\
\hline TM & TMACS & & .90 & \\
\hline PCS & PUMP CONTROL STA & & 66.4 & \\
\hline & & & & \\
\hline & & & & \\
\hline
\end{tabular}

TEST EQUIP \# 785-77-47-001

CALIB DUE DATE 3-12-97 


\section{RISER RESISTANCE DATA SHEET}

TANK T-202

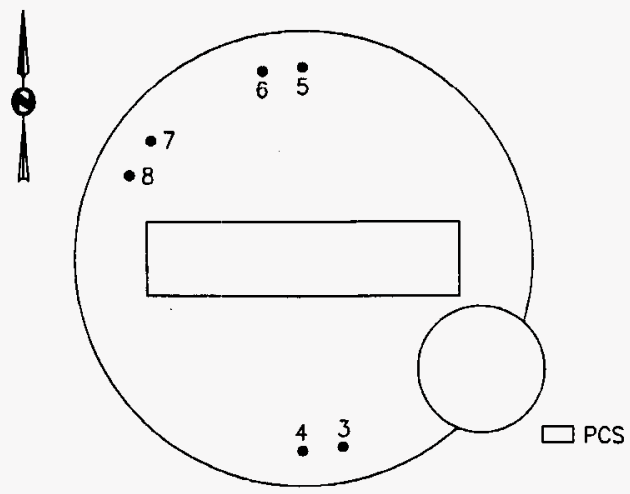

\begin{tabular}{|l|l|c|c|l|}
\hline \multirow{2}{*}{ RISER } & \multirow{2}{*}{ DESCRIPTION } & \multicolumn{2}{c|}{ RESISTANCE(OHMS) } & \multirow{2}{*}{ COMMENTS } \\
\cline { 3 - 4 } & & RISER & TOP & \\
\hline \hline 3 & $12 "$ SPARE & 39.6 & 2060 & U.G.R. \\
\hline 4 & $4 "$ LIQ LEVEL REEL & 8030 & OPEN & U.G.R. \\
\hline 6 & $12 "$ BREATHER & 1.04 & 1.05 & \\
\hline 5 & $4 "$ TEMP PROBE & 1.02 & .91 & \\
\hline 7 & $12 "$ OBSV PORT & 1.14 & 1.18 & \\
\hline 8 & $4 "$ SPARE & OPEN & OPEN & U.G.R. \\
\hline PCS & PUMP CONTROL STA & & 73.2 & \\
\hline & & & & \\
\hline & & & & \\
\hline & & & & \\
\hline
\end{tabular}

TEST EQUIP \# 785-77-47-001

CALIB DUE DATE $3-12-97$ 


\section{RISER RESISTANCE DATA SHEET}

TANK T-203

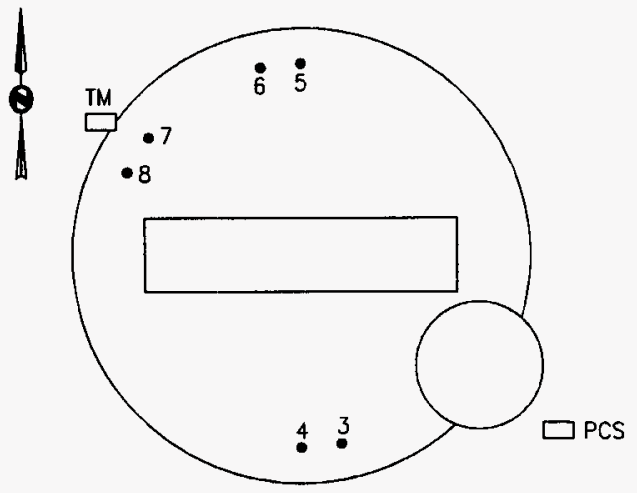

\begin{tabular}{|c|c|c|c|c|}
\hline \multirow{2}{*}{ RISER } & \multirow{2}{*}{ DESCRIPTION } & \multicolumn{2}{|c|}{ RESISTANCE(OHMS) } & \multirow{2}{*}{ COMMENTS } \\
\hline & & RISER & TOP & \\
\hline 3 & 12" SPARE & 1.1 & 746 & \\
\hline 4 & 4" LIQ LEVEL REEL & $20 K$ & OPEN & U.G.R. \\
\hline 5 & 4" TEMP PROBE & 60.7 & 1140 & U.G.R. \\
\hline 6 & 12" BREATHER & 1.09 & 1.15 & \\
\hline 7 & 12" OBSV PORT & 1.10 & 1.37 & \\
\hline 8 & 4" THERMOCOUPLE & 1.08 & .92 & G.T.W. \\
\hline TM & TMACS & & .88 & \\
\hline PCS & PUMP CONTROL STA & & 25.9 & \\
\hline & & & & \\
\hline & & & & \\
\hline
\end{tabular}

TEST EQUIP \# 785-77-47-001

CALIB DUE DATE 3-12-97 


\section{RISER RESISTANCE DATA SHEET}

TANK T-204

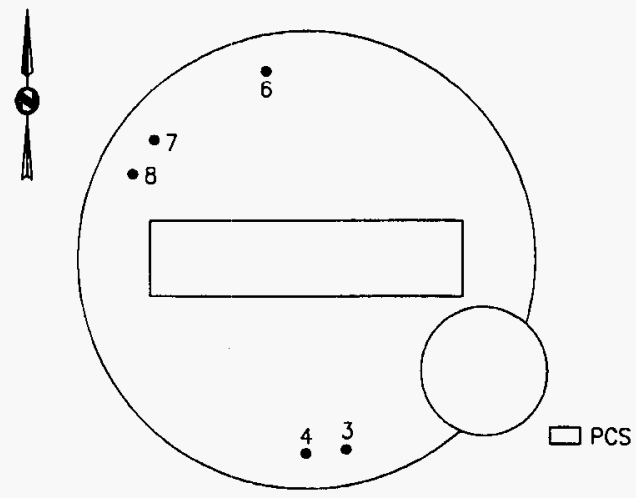

\begin{tabular}{|l|l|c|c|l|}
\hline \multirow{2}{*}{ RISER } & \multirow{2}{*}{ DESCRIPTION } & \multicolumn{2}{|c|}{ RESISTANCE(OHMS) } & \multirow{2}{*}{ COMMENTS } \\
\cline { 3 - 4 } & & RISER & TOP & \\
\hline 3 & $12 "$ DIP TUBES & 40.0 & 41.5 & U.G.R. \\
\hline 4 & $4 "$ LIQ LEVEL REEL & 91.9 & OPEN & U.G.R. \\
\hline 6 & $12 "$ BREATHER & 38.3 & 39.5 & U.G.R. \\
\hline 7 & $12 "$ OBSV PORT & 53.8 & 522 & U.G.R. \\
\hline 8 & $4 "$ THERMOCOUPLE & .70 & .59 & G.T.W. \\
\hline PCS & PUMP CONTROL STA & & 89.8 & \\
\hline & & & & \\
\hline & & & & \\
\hline & & & & \\
\hline & & & & \\
\hline
\end{tabular}

TEST EQUIP \# 785-77-47-001

CALIB DUE DATE 3-12-97 
WHC-SD-WM-TR-034, REV. 0

\section{RISER RESISTANCE DATA SHEET}

TANK TX-101

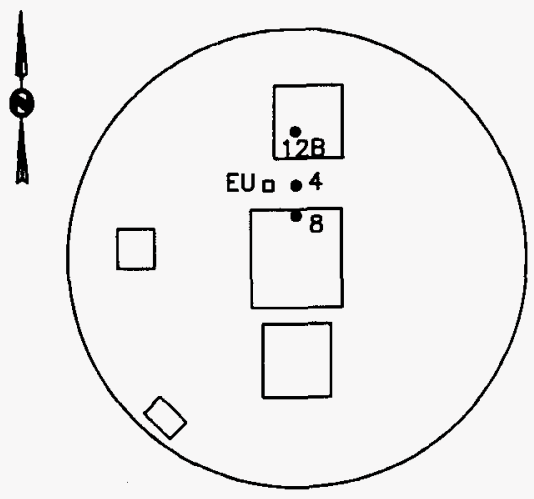

\begin{tabular}{|c|l|c|c|c|}
\hline \multirow{2}{*}{ RISER } & \multirow{2}{*}{ DESCRIPTION } & \multicolumn{2}{|c|}{ RESISTANCE(OHMS) } & \multirow{2}{*}{ COMMENTS } \\
\cline { 3 - 5 } & & RISER & TOP & \\
\hline \hline 4 & $4 "$ ENRAF & 2.51 & 2.60 & \\
\hline 8 & $4 "$ BREATHER & 2.49 & 2.51 & \\
\hline 128 & $12 "$ OBSV PORT & 30.7 & 941 & U.G.R. \\
\hline EU & ENRAF UNISTRUT & & 2.63 & \\
\hline & & & & \\
\hline & & & & \\
\hline & & & & \\
\hline & & & & \\
\hline & & & & \\
\hline & & & & \\
\hline
\end{tabular}

TEST EQUIP \# 785-77-47-001

CALIB DUE DATE $3-12-97$ 


\section{RISER RESISTANCE DATA SHEET}

TANK TX-102

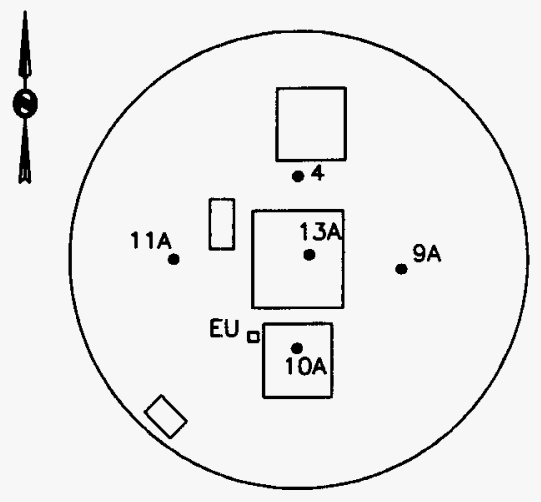

\begin{tabular}{|c|c|c|c|c|}
\hline \multirow{2}{*}{ RISER } & \multirow{2}{*}{ DESCRIPTION } & \multicolumn{2}{|c|}{ RESISTANCE(OHMS) } & \multirow{2}{*}{ COMMENTS } \\
\hline & & RISER & TOP & \\
\hline 4 & 4" TEMP PROBE & 2.46 & 2.46 & \\
\hline $9 \mathrm{~A}$ & 4" BREATHER & 2.46 & 2.46 & \\
\hline $10 \mathrm{~A}$ & 4" LLR TAPE & 17.94 & OPEN & U.G.R \\
\hline $11 \mathrm{~A}$ & 4" FIBERGLASS LOW & 2.81 & & \\
\hline $13 A$ & 12" OBSV PORT & 2.49 & 2.60 & \\
\hline EU & ENRAF UNISTRUT & & 490 & \\
\hline & & & & \\
\hline & & & & \\
\hline & & & & \\
\hline & & & & \\
\hline
\end{tabular}

TEST EQUIP \# 785-77-47-001 CALIB DUE DATE 3-12-97 
WHC-SD-WM-TR-034, REV. 0

\section{RISER RESISTANCE DATA SHEET}

TANK TX-103

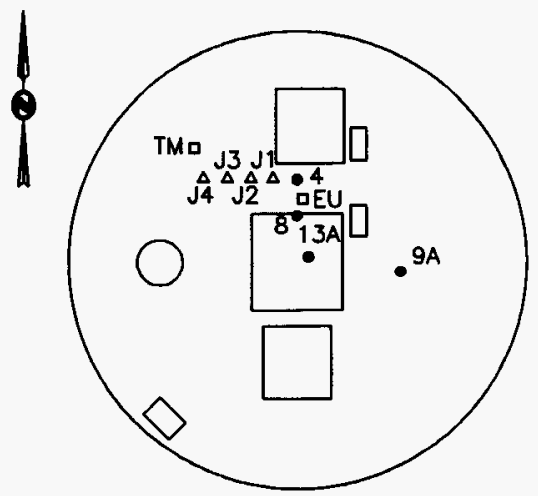

\begin{tabular}{|c|l|c|c|c|}
\hline \multirow{2}{*}{ RISER } & \multicolumn{1}{|c|}{ DESCRIPTION } & \multicolumn{2}{c|}{ RESISTANCE(OHMS) } & \multirow{2}{*}{ COMMENTS } \\
\cline { 3 - 4 } & & RISER & TOP & \\
\hline 4 & $4 "$ TEMP PROBE & .55 & .55 & \\
\hline 8 & $4 "$ ENRAF & .55 & .67 & \\
\hline 9A & $4 "$ BREATHER & .56 & .56 & \\
\hline 13A & $12 "$ OBSV PORT & .55 & .55 & \\
\hline EU & ENRAF UNISTRUT & & .66 & \\
\hline J1 & $8 \times 8 \times 6$ JB & & .67 & \\
\hline J2 & $8 \times 8 \times 6$ JB & & .70 & \\
\hline J3 & $8 \times 8 \times 6$ JB & & .65 & \\
\hline J4 & $8 \times 8 \times 6$ JB & & .68 & \\
\hline TM & TMACS & & .67 & \\
\hline
\end{tabular}

TEST EQUIP \# 785-77-47-001

CALIB DUE DATE 3-12-97 


\section{RISER RESISTANCE DATA SHEET}

TANK TX-104

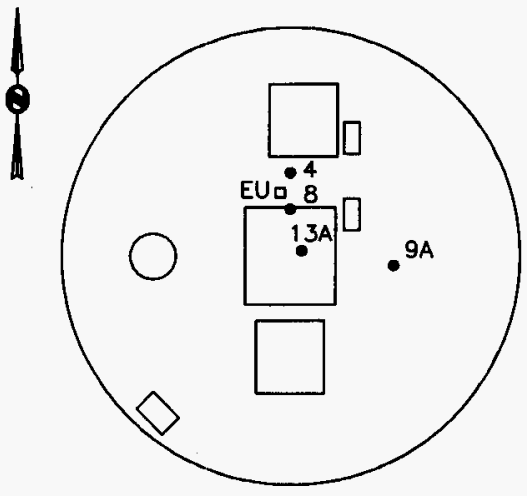

\begin{tabular}{|c|l|c|c|c|}
\hline \multirow{2}{*}{ RISER } & \multirow{2}{*}{ DESCRIPTION } & \multicolumn{2}{c|}{ RESISTANCE(OHMS) } & \multirow{2}{*}{ COMMENTS } \\
\cline { 1 - 3 } & & RISER & TOP & \multirow{2}{*}{ CO } \\
\hline 4 & $4 "$ TEMP PROBE & .89 & .89 & \\
\hline 8 & $4 "$ ENRAF & .57 & .85 & \\
\hline 9A & $4 "$ BREATHER & .54 & .54 & \\
\hline $13 \mathrm{~A}$ & $12^{\prime \prime}$ OBSV PORT & 4.68 & 4.68 & \\
\hline EU & ENRAF UNISTRUT & & .84 & \\
\hline & & & & \\
\hline & & & & \\
\hline & & & & \\
\hline & & & & \\
\hline & & & & \\
\hline
\end{tabular}

TEST EQUIP \# 785-77-47-001 CALIB DUE DATE 3-12-97 


\section{RISER RESISTANCE DATA SHEET}

TANK TX-105

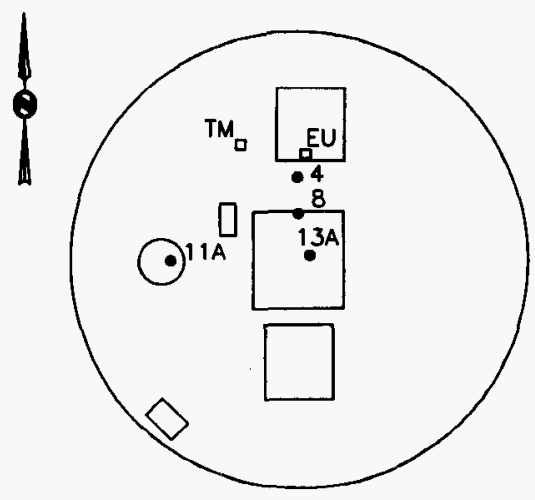

\begin{tabular}{|c|l|c|c|c|}
\hline \multirow{2}{*}{ RISER } & DESCRIPTION & \multicolumn{2}{c|}{ RESISTAN } & \multirow{2}{*}{ COMMENTS } \\
\cline { 3 - 4 } & & RISER & TOP & \multirow{2}{*}{} \\
\hline 4 & $4 "$ CAPPED RISER & 2.74 & 2.74 & \\
\hline 8 & $4 "$ LIQ LEVEL REEL & 3.12 & OPEN & \\
\hline $11 \mathrm{~A}$ & $4 "$ BREATHER & 2.78 & 2.82 & \\
\hline $13 \mathrm{~A}$ & $12^{\prime \prime}$ OBSV PORT & 2.52 & 3.10 & \\
\hline EU & ENRAF UNISTRUT & & 2.66 & \\
\hline TM & TMACS & & 3.69 & \\
\hline & & & & \\
\hline & & & & \\
\hline & & & & \\
\hline & & & & \\
\hline
\end{tabular}

TEST EQUIP \# 785-77-47-001

CALIB DUE DATE $3-12-97$ 


\section{RISER RESISTANCE DATA SHEET}

TANK TX-106

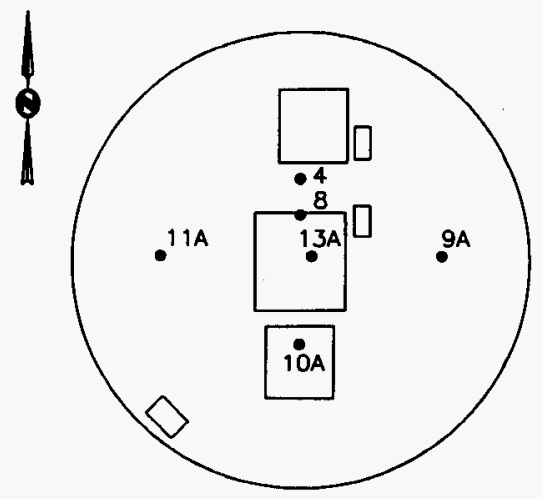

\begin{tabular}{|c|c|c|c|c|}
\hline \multirow{2}{*}{ RISER } & \multirow{2}{*}{ DESCRIPTION } & \multicolumn{2}{|c|}{ RESISTANCE(OHMS) } & \multirow{2}{*}{ COMMENTS } \\
\hline & & RISER & TOP & \\
\hline 4 & 4" TEMP PROBE & .52 & .52 & \\
\hline 8 & 4" LIQ LEVEL REEL & .50 & .91 & \\
\hline \begin{tabular}{|l|}
$9 A$ \\
\end{tabular} & 4" SPARE & .47 & .52 & \\
\hline $10 \mathrm{~A}$ & $4^{\prime \prime}$ BREATHER & $4.7 \mathrm{~K}$ & $4.7 \mathrm{~K}$ & U.G.R. \\
\hline $11 \mathrm{~A}$ & 4" FIBERGLASS LOW & .53 & & \\
\hline $13 A$ & 12" OBSV PORT & .64 & .64 & \\
\hline & & & & \\
\hline & & & & \\
\hline & & & & \\
\hline & & & & \\
\hline & & $\begin{array}{l}\text { TEST } \\
\text { CALI }\end{array}$ & $\begin{array}{l}\text { UIP \# } \\
\text { UE DAT }\end{array}$ & $\frac{785-77-47-001}{3-12-97}$ \\
\hline
\end{tabular}




\section{RISER RESISTANCE DATA SHEET}

TANK TX-107

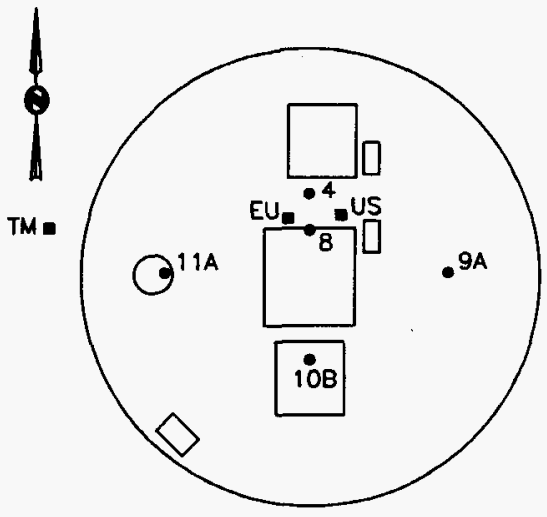

\begin{tabular}{|c|c|c|c|c|}
\hline \multirow{2}{*}{ RISER } & \multirow{2}{*}{ DESCRIPTION } & \multicolumn{2}{|c|}{ RESISTANCE(OHMS) } & \multirow{2}{*}{ COMMENTS } \\
\hline & & RISER & TOP & \\
\hline 4 & 4" TEMP PROBE & .70 & .70 & \\
\hline 8 & 4" FIC & .61 & .61 & \\
\hline $9 A$ & 4" SPARE & .71 & .71 & \\
\hline $10 \mathrm{~B}$ & 12" OBSV PORT & 6.02 & 6.02 & U.G.R. \\
\hline $11 \mathrm{~A}$ & 4" BREATHER & .58 & .58 & \\
\hline EU & ENRAF UNISTRUT & & $6.7 \mathrm{~K}$ & \\
\hline TM & TMACS & & .59 & \\
\hline US & UNISTRUT STAND & & .77 & \\
\hline & & & & \\
\hline & & & & \\
\hline
\end{tabular}

TEST EQUIP \# 785-77-47-001

CALIB DUE DATE 3-12-97 
WHC-SD-WM-TR-034, REV. 0

\section{RISER RESISTANCE DATA SHEET}

TANK TX-108

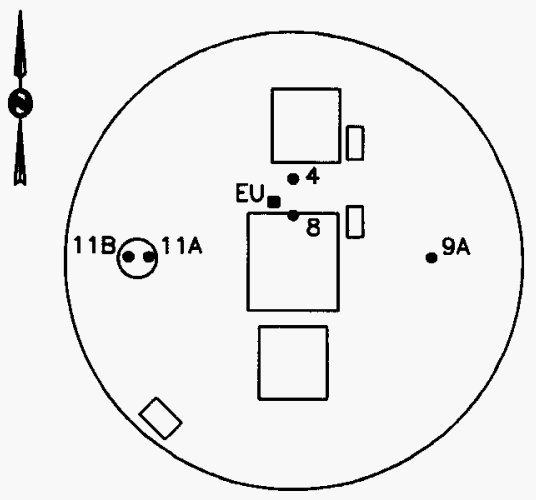

\begin{tabular}{|c|l|c|c|l|}
\hline \multirow{2}{*}{ RISER } & \multirow{2}{*}{ DESCRIPTION } & \multicolumn{2}{c|}{ RESISTANCE(OHMS) } & \multirow{2}{*}{ COMMENTS } \\
\cline { 3 - 4 } & & RISER & TOP & \\
\hline \hline 4 & $4 "$ TEMP PROBE & 1.03 & 1.03 & \\
\hline 8 & $4 "$ FIC & .64 & .64 & \\
\hline 9A & $4 "$ SMP LOW & 5.46 & & U.G.R. \\
\hline $11 \mathrm{~A}$ & $4 "$ BREATHER & 1.03 & 1.03 & \\
\hline $11 \mathrm{~B}$ & $12 "$ OBSV PORT & 1.03 & 1.03 & \\
\hline EU & ENRAF UNISTRUT & & $6.5 \mathrm{~K}$ & \\
\hline & & & & \\
\hline & & & & \\
\hline & & & & \\
\hline & & & & \\
\hline
\end{tabular}

TEST EQUIP \# 785-77-47-001

CALIB DUE DATE 3-12-97 


\section{RISER RESISTANCE DATA SHEET}

TANK TX-109

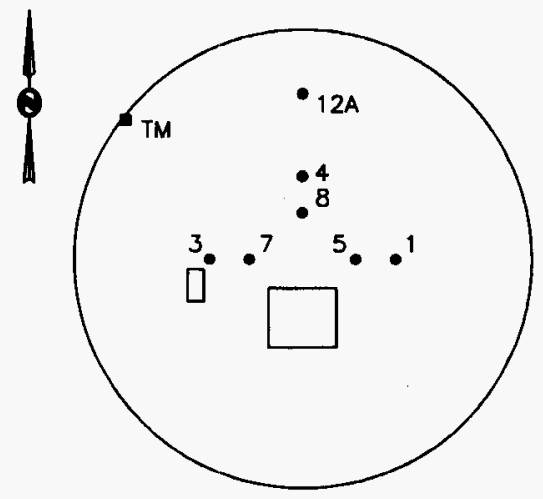

\begin{tabular}{|c|c|c|c|c|}
\hline \multirow{2}{*}{ RISER } & \multirow{2}{*}{ DESCRIPTION } & \multicolumn{2}{|c|}{ RESISTANCE(OHMS) } & \multirow{2}{*}{ COMMENTS } \\
\hline & & RISER & TOP & \\
\hline 1 & 4" LIQ LEVEL REEL & .57 & $.75(P)$ & PEDESTAL \\
\hline 3 & 4" FIBERGLASS LOW & .27 & & \\
\hline 4 & 4" ENRAF & .54 & .87 & \\
\hline 5 & $12 "$ SPARE & .55 & 1.37 & \\
\hline 7 & 12" OBSV PORT & .27 & .27 & \\
\hline 8 & 4" TEMP PROBE & .53 & 1.09 & \\
\hline $12 \mathrm{~A}$ & 4" BREATHER & .88 & .88 & \\
\hline TM & TMACS & & .41 & \\
\hline & & & & \\
\hline & & & & \\
\hline
\end{tabular}

TEST EQUIP \# 785-77-47-001

CALIB DUE DATE 3-12-97 


\section{RISER RESISTANCE DATA SHEET}

TANK TX-110

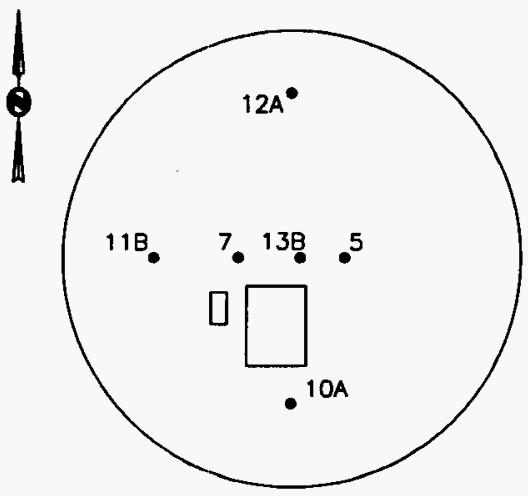

\begin{tabular}{|c|c|c|c|c|}
\hline \multirow{2}{*}{ RISER } & \multirow{2}{*}{ DESCRIPTION } & \multicolumn{2}{|c|}{ RESISTANCE(OHMS) } & \multirow{2}{*}{ COMMENTS } \\
\hline & & RISER & TOP & \\
\hline 5 & 12" OBSV PORT & .69 & .69 & \\
\hline 7 & 12" LIQ LEVEL REEL & .48 & $.48(P)$ & PEDESTAL \\
\hline $10 A$ & 4" SPARE & .49 & .49 & \\
\hline 11B & 4" SPARE & .63 & 4.05 & \\
\hline $12 \mathrm{~A}$ & 4" BREATHER & .44 & .44 & \\
\hline $13 \mathrm{~B}$ & 4" FIBERGLASS LOW & 1.06 & & \\
\hline & & & & \\
\hline & & & & \\
\hline & & & & \\
\hline & & & & \\
\hline
\end{tabular}

TEST EQUIP \# 785-77-47-001

CALIB DUE DATE 3-12-97 


\section{RISER RESISTANCE DATA SHEET}

TANK TX-111

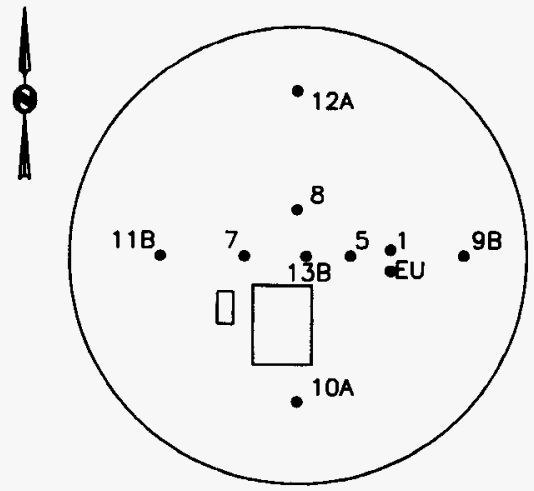

\begin{tabular}{|c|c|c|c|c|}
\hline \multirow{2}{*}{ RISER } & \multirow{2}{*}{ DESCRIPTION } & \multicolumn{2}{|c|}{ RESISTANCE(OHMS) } & \multirow{2}{*}{ COMMENTS } \\
\hline & & RISER & TOP & \\
\hline 1 & 4" LIQ LEVEL REEL & .49 & .49 & \\
\hline 5 & $12^{\prime \prime}$ SPARE & .50 & .50 & \\
\hline 7 & 12" OBSV PORT & .58 & .58 & \\
\hline 8 & 4" TEMP PROBE & .54 & .54 & \\
\hline $9 \mathrm{~B}$ & 4" SPARE & .52 & 140 & \\
\hline $10 \mathrm{~A}$ & 4" SPARE & .54 & .54 & \\
\hline 118 & 4" FIBERGLASS LOW & 2.33 & & \\
\hline $12 \mathrm{~A}$ & 4" BREATHER & .51 & .51 & \\
\hline $13 \mathrm{~B}$ & 4" SPARE & .54 & .54 & \\
\hline EU & ENRAF UNISTRUT & & 4.83 & \\
\hline
\end{tabular}

TEST EQUIP \# 785-77-47-001

CALIB DUE DATE 3-12-97 
WHC-SD-WM-TR-034, REV. 0

\section{RISER RESISTANCE DATA SHEET}

TANK TX-112

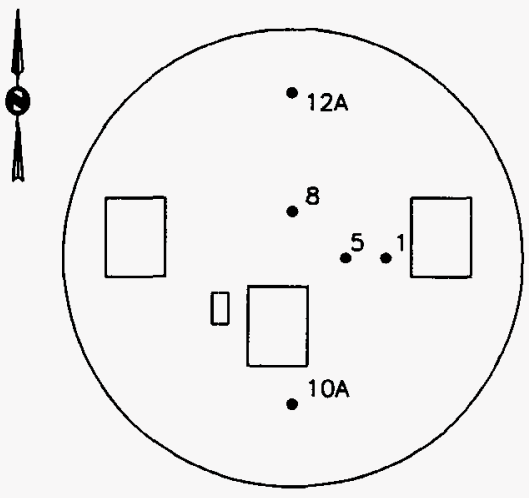

\begin{tabular}{|c|c|c|c|c|}
\hline \multirow{2}{*}{ RISER } & \multirow{2}{*}{ DESCRIPTION } & \multicolumn{2}{|c|}{ RESISTANCE(OHMS) } & \multirow{2}{*}{ COMMENTS } \\
\hline & & RISER & TOP & \\
\hline 1 & 4" LIQ LEVEL REEL & .82 & OPEN & \\
\hline 5 & 12" OBSV PORT & .51 & 98.9 & \\
\hline 8 & 4" TEMP PROBE & .56 & .56 & \\
\hline $10 \mathrm{~A}$ & 4" BREATHER & 4.36 & 4.45 & \\
\hline $12 \mathrm{~A}$ & 4" FIBERGLASS LOW & .56 & & \\
\hline & & & & \\
\hline & & & & \\
\hline & & & & \\
\hline & & & & \\
\hline & & & & \\
\hline
\end{tabular}

TEST EQUIP \# 785-77-47-001 CALIB DUE DATE 3-12-97 


\section{RISER RESISTANCE DATA SHEET}

TANK TX-113

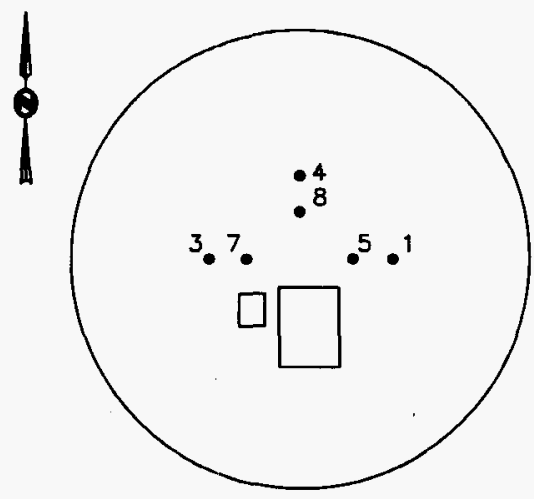

\begin{tabular}{|c|l|c|c|c|}
\hline \multirow{2}{*}{ RISER } & \multirow{2}{*}{ DESCRIPTION } & \multicolumn{2}{|c|}{ RESISTANCE(OHMS) } & \multirow{2}{*}{ COMMENTS } \\
\cline { 3 - 5 } & & RISER & TOP & \\
\hline 1 & $4 "$ & .33 & .36 & \\
\hline 3 & $4 "$ SIQ LEVEL REEL & .31 & .33 & \\
\hline 4 & $4 "$ FIBERGLASS LOW & .30 & & \\
\hline 5 & $12 "$ OBSV PORT & .32 & .34 & \\
\hline 7 & $12 "$ BREATHER & .28 & .41 & \\
\hline 8 & $4 "$ TEMP PROBE & .33 & .35 & \\
\hline & & & & \\
\hline & & & & \\
\hline & & & & \\
\hline & & & & \\
\hline
\end{tabular}

TEST EQUIP \# 785-77-47-001

CALIB DUE DATE 3-12-97 


\section{RISER RESISTANCE DATA SHEET}

TANK TX-114

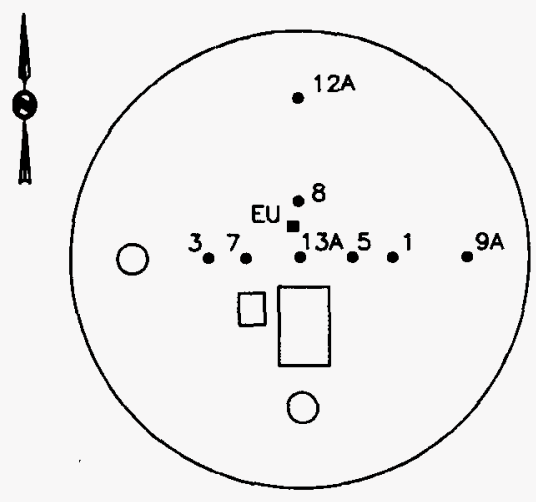

\begin{tabular}{|c|l|c|c|c|}
\hline \multirow{2}{*}{ RISER } & \multirow{2}{*}{ DESCRIPTION } & \multicolumn{2}{c|}{ RESISTANCE(OHMS) } & \multirow{2}{*}{ COMMENTS } \\
\cline { 3 - 4 } & & RISER & TOP & \\
\hline 1 & $4 "$ BREATHER & .49 & .49 & \\
\hline 3 & $4 "$ SPARE & .49 & OPEN & \\
\hline 5 & $12 "$ OBSV PORT & .41 & .46 & \\
\hline 7 & $12 "$ FIBERGLASS LOW & .49 & & \\
\hline 8 & $4 "$ LIQ LEVEL REEL & .45 & $1.41 \mathrm{~K}$ & \\
\hline $9 \mathrm{~A}$ & $4 "$ SPARE & 10.9 & 10.9 & U.G.R. \\
\hline $12 \mathrm{~A}$ & $4 "$ SPARE & .49 & $1 \mathrm{~K}$ & \\
\hline 13 & $4 "$ SPARE & .31 & .33 & \\
\hline EU & ENRAF UNISTRUT & & $1.28 \mathrm{~K}$ & \\
\hline & & & & \\
\hline & & & & \\
\hline & & & & \\
\hline
\end{tabular}

TEST EQUIP \# 785-77-47-001 CALIB DUE DATE 3-12-97 
WHC-SD-WM-TR-034, REV. 0

\section{RISER RESISTANCE DATA SHEET}

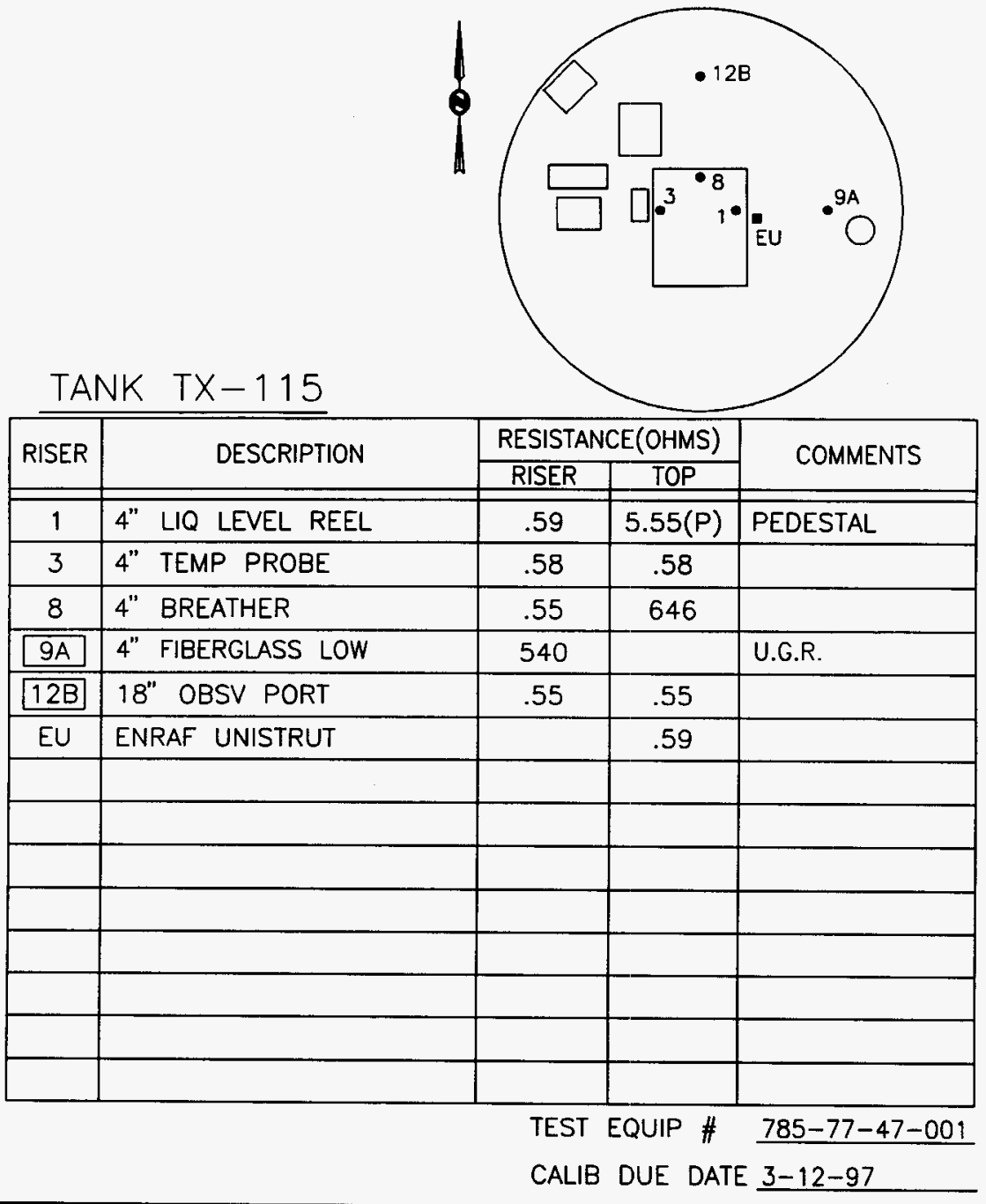




\section{RISER RESISTANCE DATA SHEET}

TANK TX-116

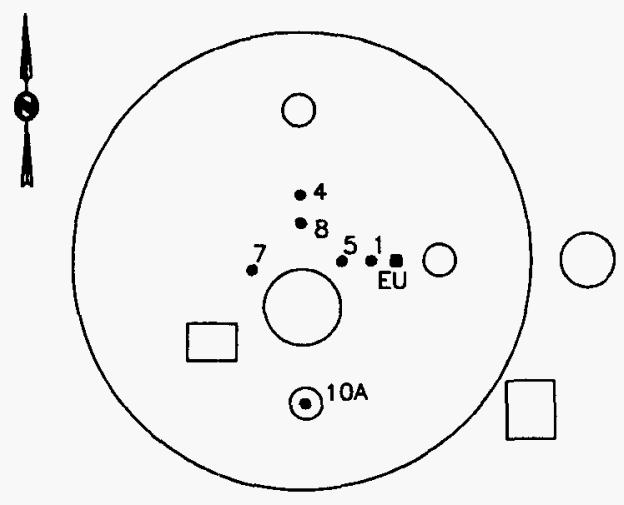

\begin{tabular}{|c|l|c|c|c|}
\hline \multirow{2}{*}{ RISER } & \multirow{2}{*}{ DESCRIPTION } & \multicolumn{2}{c|}{ RESISTANCE(OHMS) } & \multirow{2}{*}{ COMMENTS } \\
\cline { 3 - 4 } & & RISER & TOP & \\
\hline 1 & $4 "$ LIQ LEVEL REEL & .92 & & \\
\hline 4 & $\begin{array}{l}4^{\prime \prime} \text { FLANGE TEMP } \\
\text { CUT IN RISER }\end{array}$ & .90 & 1.01 & \\
\hline 5 & $12 "$ OBSV PORT & .91 & .92 & \\
\hline 7 & ENRAF UNISTRUT & .92 & 1.04 & \\
\hline 8 & $\begin{array}{l}\text { 4" FLANGE TEMP } \\
\text { CUT IN RISER }\end{array}$ & .91 & 1.01 & \\
\hline $10 A$ & $4 "$ BREATHER & 334 & 338 & U.G.R. \\
\hline EU & ENRAF UNISTRUT & & 492 & \\
\hline & & & & \\
\hline & & & & \\
\hline & & & & \\
\hline
\end{tabular}

TEST EQUIP \# 785-77-47-001

CALIB DUE DATE 3-12-97 
WHC-SD-WM-TR-034, REV. 0

\section{RISER RESISTANCE DATA SHEET}

TANK TX-117

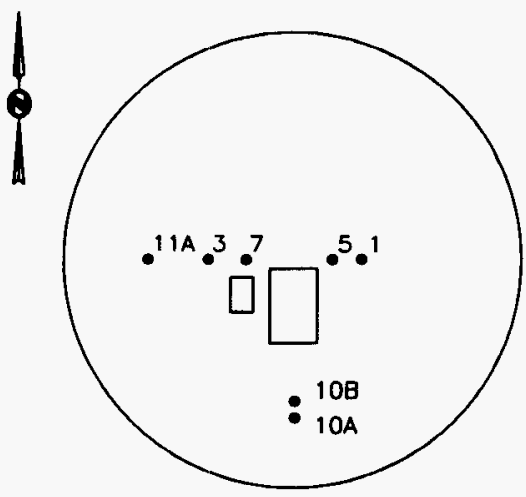

\begin{tabular}{|c|l|c|c|l|}
\hline \multirow{2}{*}{ RISER } & \multirow{2}{*}{ DESCRIPTION } & \multicolumn{2}{|c|}{ RESISTANCE(OHMS) } & \multirow{2}{*}{ COMMENTS } \\
\cline { 3 - 4 } & & RISER & TOP & \\
\hline 1 & $4 "$ SPARE & .90 & .91 & \\
\hline 3 & $4 "$ SPARE & .90 & .90 & \\
\hline 5 & $12 "$ OBSV PORT & .89 & 498 & \\
\hline 7 & $12^{\prime \prime}$ FIBERGLASS LOW & 2.53 & & \\
\hline $10 \mathrm{~A}$ & $4 "$ SPARE & 90 & 90 & U.G.R. \\
\hline $10 \mathrm{~B}$ & $12 "$ BREATHER & 90 & 106.9 & U.G.R. \\
\hline $11 \mathrm{~A}$ & $4 "$ LIQ LEVEL REEL & 459 & OPEN & U.G.R. \\
\hline & & & & \\
\hline & & & & \\
\hline & & & & \\
\hline
\end{tabular}

TEST EQUIP \# 785-77-47-001 CALIB DUE DATE 3-12-97 


\section{RISER RESISTANCE DATA SHEET}

TANK TX-118
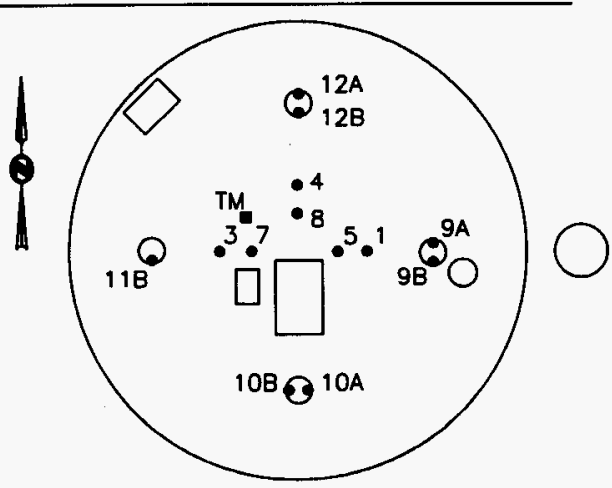

\begin{tabular}{|c|c|c|c|c|}
\hline \multirow{2}{*}{ RISER } & \multirow{2}{*}{ DESCRIPTION } & \multicolumn{2}{|c|}{ RESISTANCE(OHMS) } & \multirow{2}{*}{ COMMENTS } \\
\hline & & RISER & TOP & \\
\hline 1 & 4" THERMO-TREE & .54 & .66 & \\
\hline 3 & 4" TEMP PROBE & .52 & .52 & \\
\hline 4 & 4" ENRAF & .58 & .58 & \\
\hline 5 & $12^{n}$ FIBERGLASS LOW & .52 & & \\
\hline 7 & $12^{\prime \prime}$ SPARE & .51 & 11.22 & \\
\hline 8 & $\begin{array}{ll}4^{\prime \prime} & \text { BREATHER } \\
\end{array}$ & .57 & 1.47 & \\
\hline$\overline{\overline{9 A}}$ & 4" SPARE & .81 & .81 & \\
\hline \begin{tabular}{|c|}
$9 \mathrm{~B}$ \\
\end{tabular} & $12^{\prime \prime}$ SPARE & .75 & .75 & \\
\hline $10 \mathrm{~A}$ & 4" SPARE & .52 & .52 & \\
\hline $10 \mathrm{~B}$ & $12 "$ SPARE & .52 & .52 & \\
\hline $11 \mathrm{~B}$ & 12" OBSV PORT & .56 & .56 & \\
\hline $12 \mathrm{~A}$ & 4" SPARE & .54 & .54 & \\
\hline $12 \mathrm{~B}$ & $12^{\prime \prime}$ SPARE & .55 & .55 & \\
\hline$T M$ & TMACS & & .52 & \\
\hline & & & & \\
\hline
\end{tabular}

TEST EQUIP \# 785-77-47-001

CALIB DUE DATE 3-12-97 


\section{RISER RESISTANCE DATA SHEET}

TANK TY-101

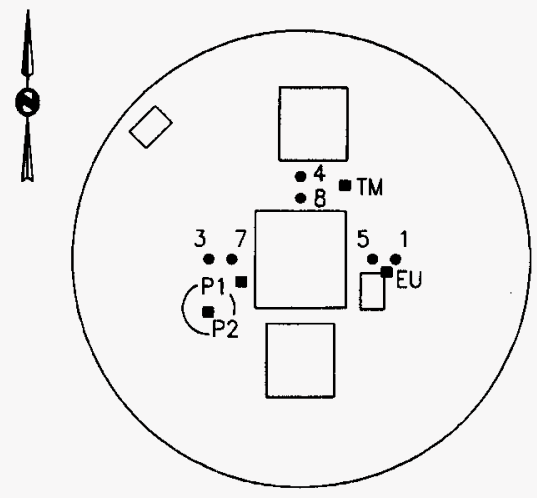

\begin{tabular}{|c|l|c|c|c|}
\hline \multirow{2}{*}{ RISER } & \multirow{2}{*}{ DESCRIPTION } & \multicolumn{2}{|c|}{ RESISTANCE(OHMS) } & \multirow{2}{*}{ COMMENTS } \\
\cline { 3 - 4 } & & RISER & TOP & \\
\hline \hline 1 & $4 "$ & .29 & .29 & \\
\hline 3 & $4 "$ TBNRAF & .27 & .27 & \\
\hline 4 & $4 "$ TEMP PROBE & .37 & .37 & \\
\hline 5 & $12 "$ OBSV PORT & .48 & .48 & \\
\hline 7 & $12 "$ BREATHER & .48 & .48 & \\
\hline 8 & $12 "$ SPARE & .48 & OPEN & \\
\hline EU & ENRAF UNISTRUT & & .29 & \\
\hline TM & TMACS & & .30 & \\
\hline P1 & 2" ELBOW & & .47 & \\
\hline P2 & $2 "$ ELBOW & & .51 & \\
\hline
\end{tabular}

TEST EQUIP \# 785-77-47-001

CALIB DUE DATE 3-12-97 


\section{RISER RESISTANCE DATA SHEET}

TANK TY-102

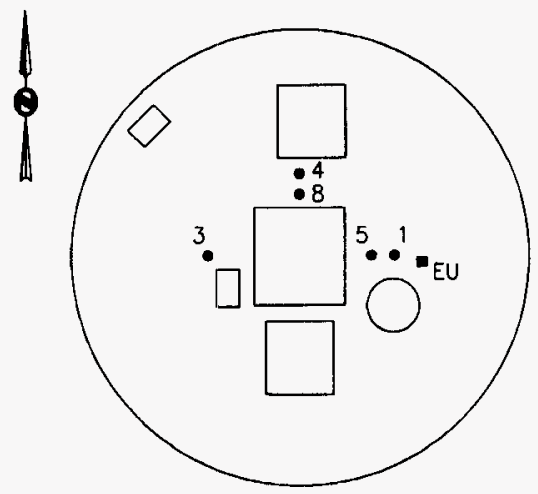

\begin{tabular}{|c|l|c|c|c|}
\hline \multirow{2}{*}{ RISER } & \multirow{2}{*}{ DESCRIPTION } & \multicolumn{2}{c|}{ RESISTANCE(OHMS) } & \multirow{2}{*}{ COMMENTS } \\
\cline { 3 - 4 } & & RISER & TOP & \\
\hline \hline 1 & $4 "$ ENRAF & .97 & .97 & \\
\hline 3 & $4 "$ SPARE & 1.92 & 1.92 & \\
\hline 4 & $4 "$ TEMP PROBE & 1.15 & 1.18 & \\
\hline 5 & $12 "$ BREATHER & 1.05 & 1.05 & \\
\hline 8 & $12 "$ SPARE & 1.04 & 1.04 & \\
\hline EU & ENRAF UNISTRUT & & 1.12 & \\
\hline & & & & \\
\hline & & & & \\
\hline & & & & \\
\hline & & & & \\
\hline
\end{tabular}

TEST EQUIP \# 785-77-47-001

CALIB DUE DATE 3-12-97 


\section{RISER RESISTANCE DATA SHEET}

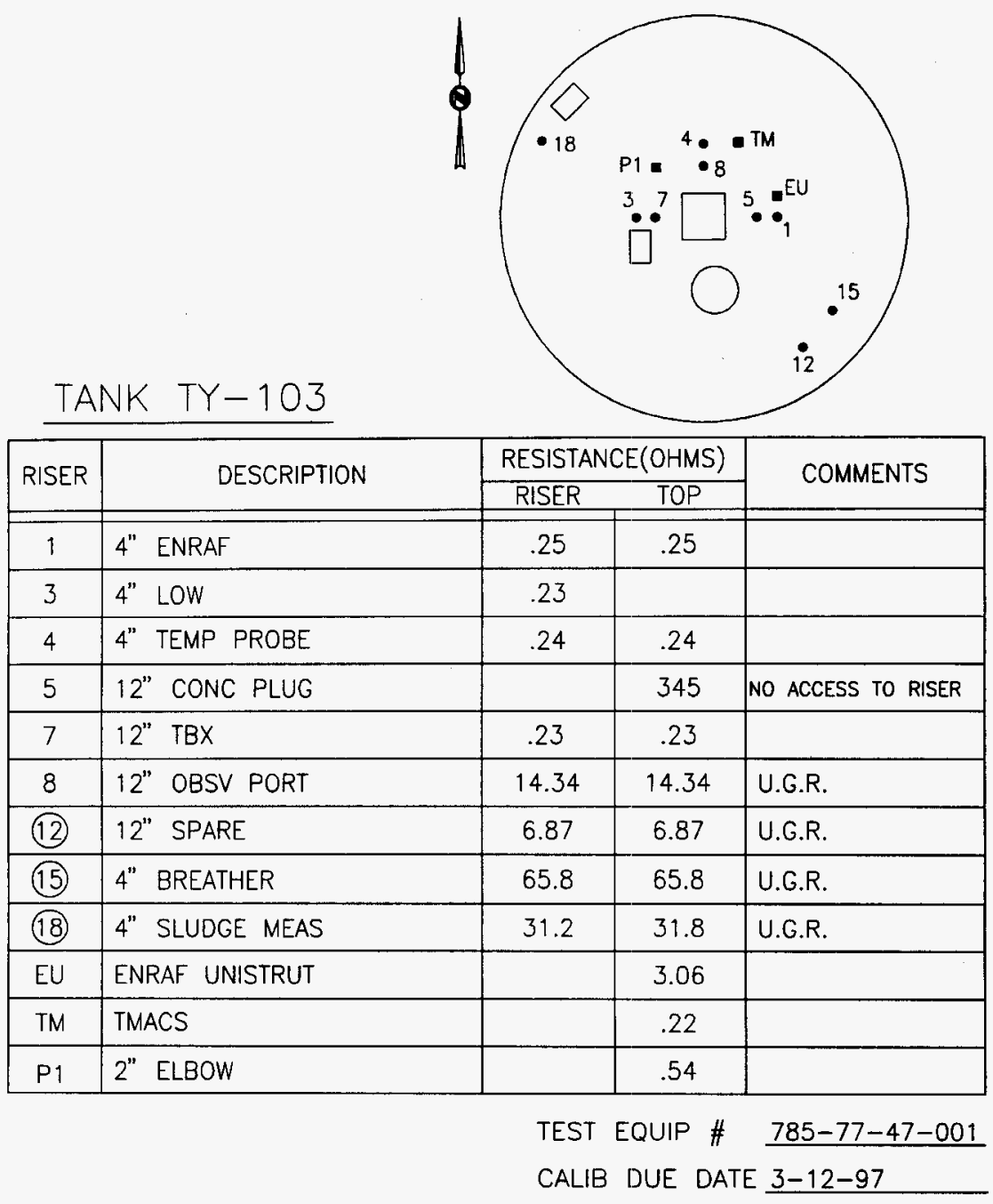




\section{RISER RESISTANCE DATA SHEET}

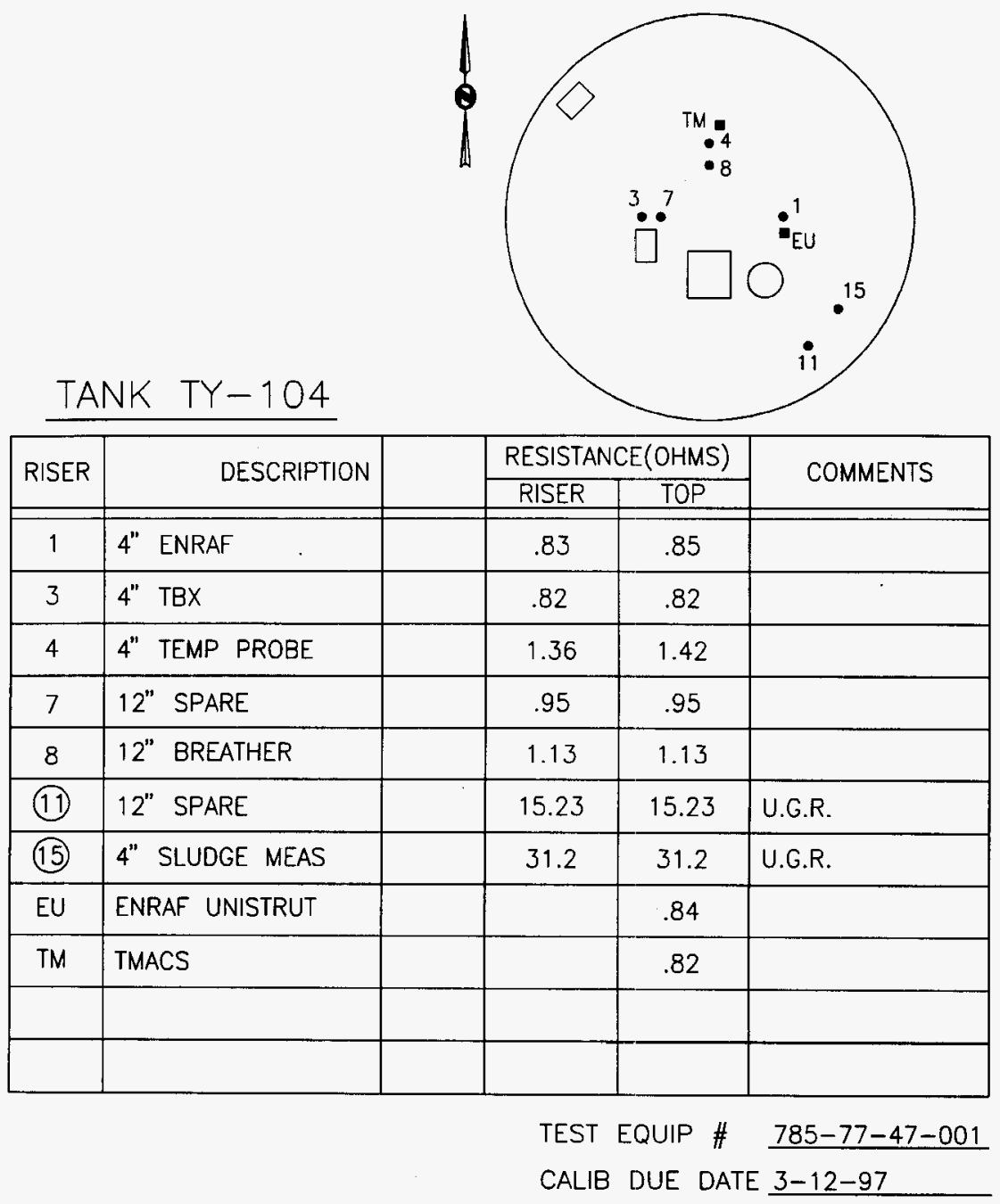




\section{RISER RESISTANCE DATA SHEET}

TANK TY-105

\begin{tabular}{|c|l|c|c|c|}
\hline \multirow{2}{*}{ RISER } & \multirow{2}{*}{ DESCRIPTION } & \multicolumn{2}{c|}{ RESISTANCE(OHMS) } & \multirow{2}{*}{ COMMENTS } \\
\cline { 3 - 4 } & & RISER & TOP & \\
\hline \hline 2 & $4 "$ & .30 & .30 & \\
\hline 3 & $4 "$ TEMARE & .29 & .29 & \\
\hline 4 & $4 "$ ENRAF & .30 & .30 & \\
\hline 6 & $12 "$ BREATHER & .31 & .31 & \\
\hline 7 & $12 "$ PLUGGED & .31 & .31 & \\
\hline 8 & $12 "$ SPARE & .30 & .30 & \\
\hline 10 & $4 "$ SPARE & 61.1 & 61.1 & U.G.R. \\
\hline 12 & $4 "$ SPARE & 105.2 & 114.7 & U.G.R. \\
\hline EU & ENRAF UNISTRUT & & .30 & \\
\hline & & & & \\
\hline & & & & \\
\hline
\end{tabular}

TEST EQUIP \# 785-77-47-001 CALIB DUE DATE $\underline{3-12-97}$ 


\section{RISER RESISTANCE DATA SHEET}

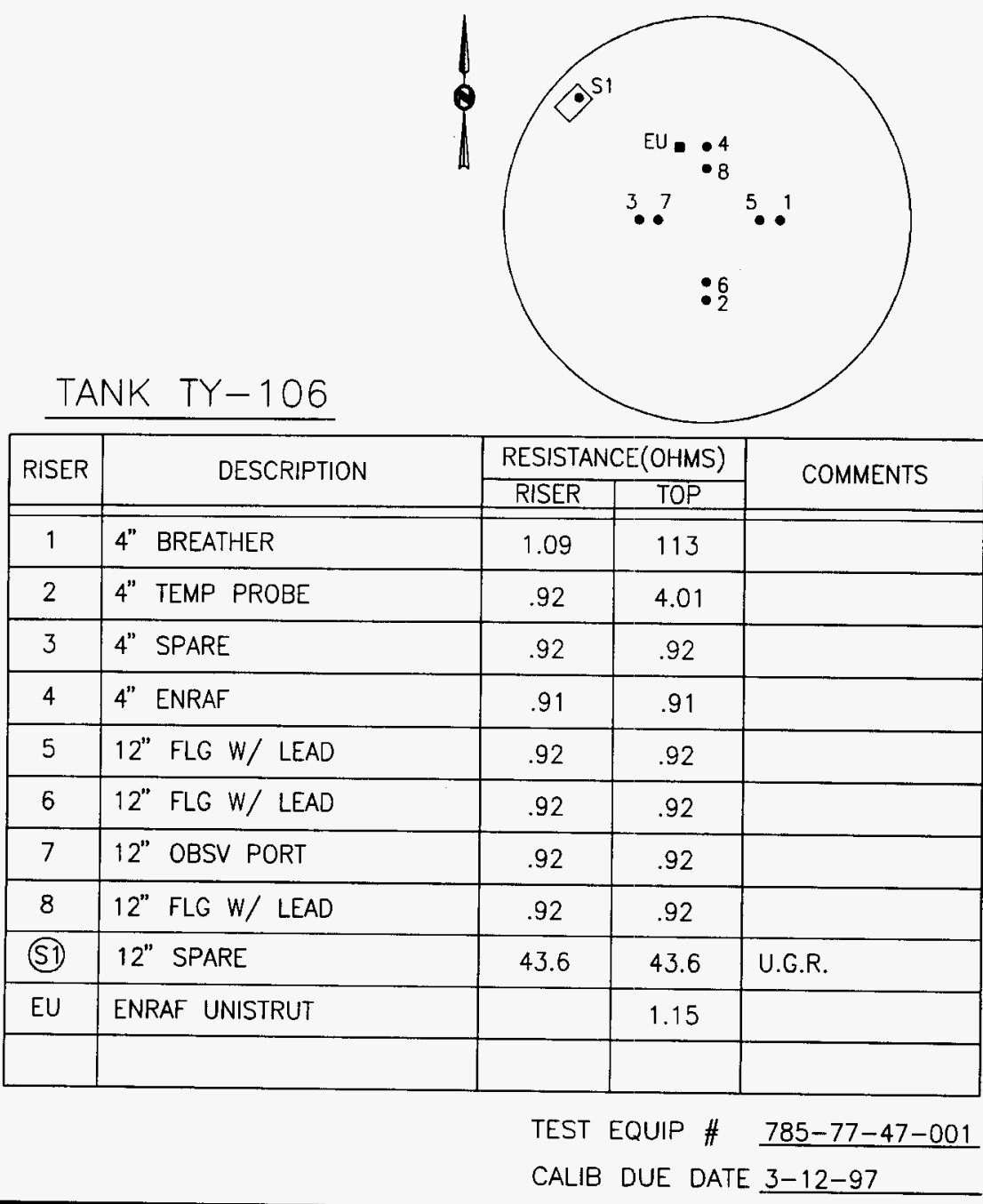




\section{RISER RESISTANCE DATA SHEET}

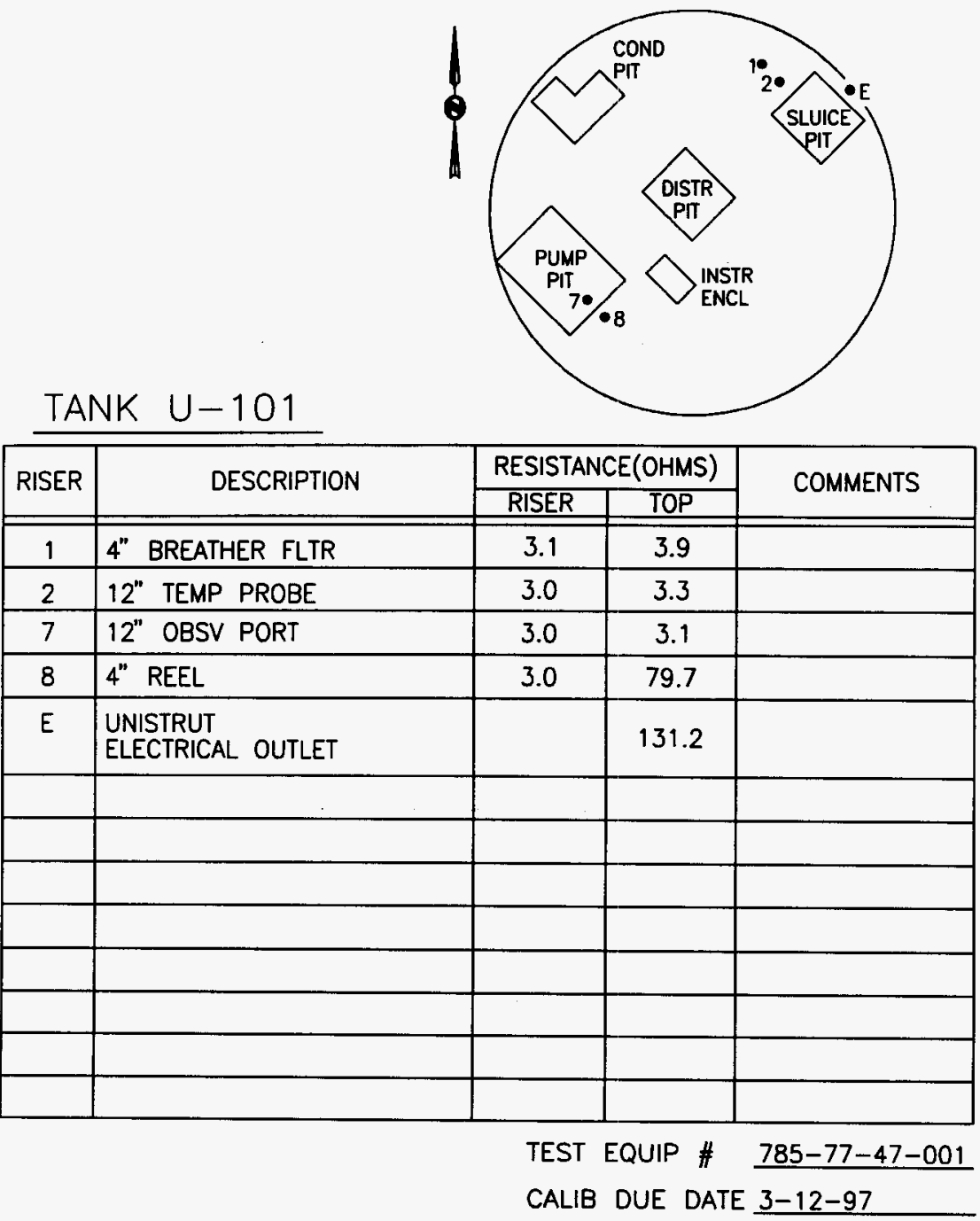




\section{RISER RESISTANCE DATA SHEET}

TANK U-102

\begin{tabular}{|c|c|c|c|c|}
\hline \multirow{2}{*}{ RISER } & \multirow{2}{*}{ DESCRIPTION } & \multicolumn{2}{|c|}{ RESISTANCE(OHMS) } & \multirow{2}{*}{ COMMENTS } \\
\hline & & RISER & TOP & \\
\hline 1 & 4" TEMP PROBE & 3.02 & 3.02 & \\
\hline 2 & $12^{\prime \prime}$ LOW & 3.02 & & \\
\hline 7 & 12" OBSV PORT & 3.00 & 3.02 & \\
\hline 8 & 4" ENRAF & 3.07 & 3.17 & \\
\hline (9) & 4" BREATHER FLTR & 3.08 & 3.08 & \\
\hline (19) & 4" SLUDGE MEAS & 40.1 & 40.6 & U.G.R. \\
\hline EU & ENRAF UNISTRUT & & 3.07 & \\
\hline LD & LEAK DET STATION & & 349 & \\
\hline LS & LEAK DET STAND & & 3.31 & \\
\hline$P C$ & PUMP CONT STA & & 3.24 & \\
\hline T1 & INSTR TUBING & & 299 & \\
\hline WFE & WF ENCLOSURE & & 3.22 & \\
\hline & & & & \\
\hline & & & & \\
\hline & & & & \\
\hline
\end{tabular}

TEST EQUIP \# 785-77-47-001

CALIB DUE DATE 3-12-97 


\section{RISER RESISTANCE DATA SHEET}

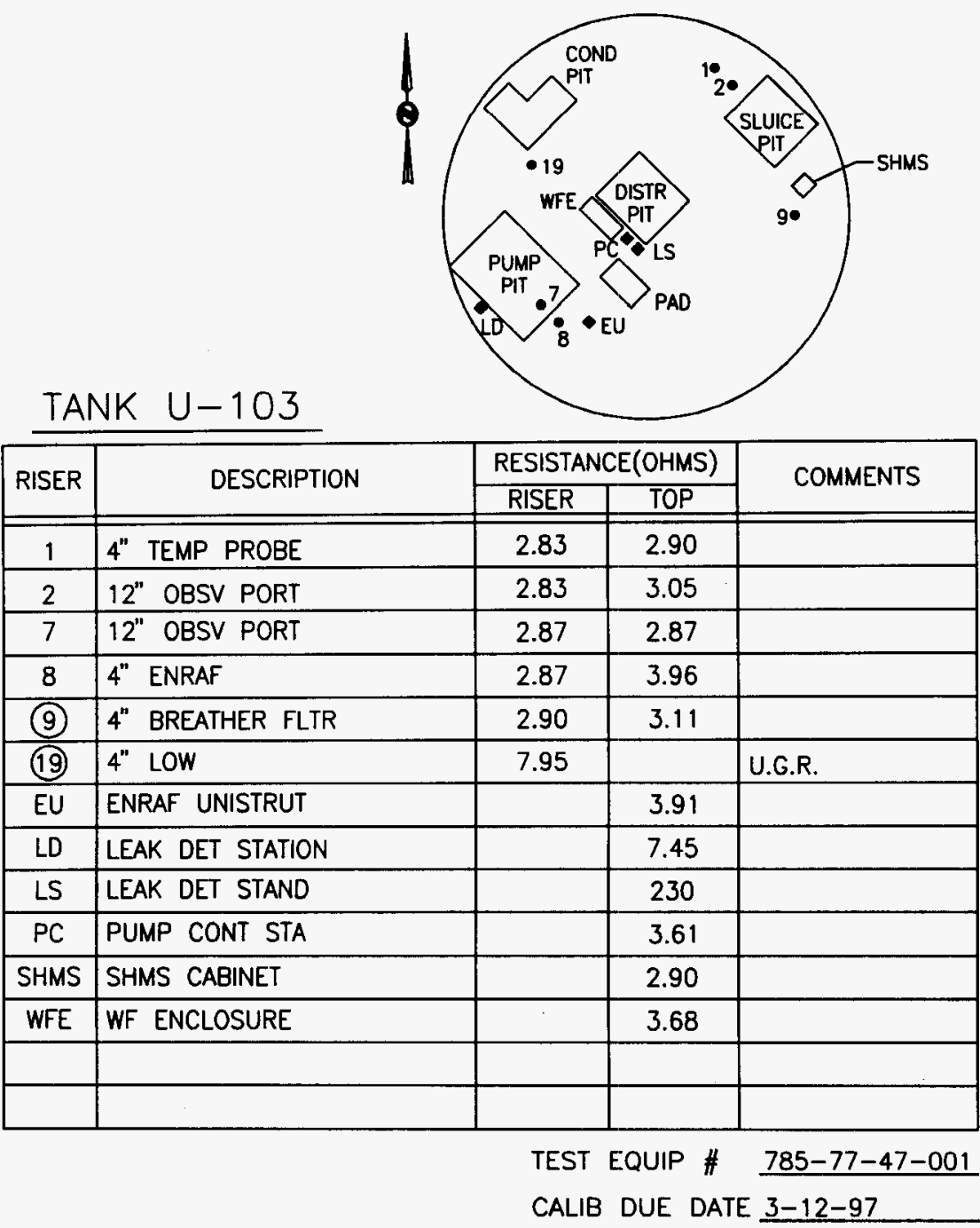




\section{RISER RESISTANCE DATA SHEET}

TANK U-104

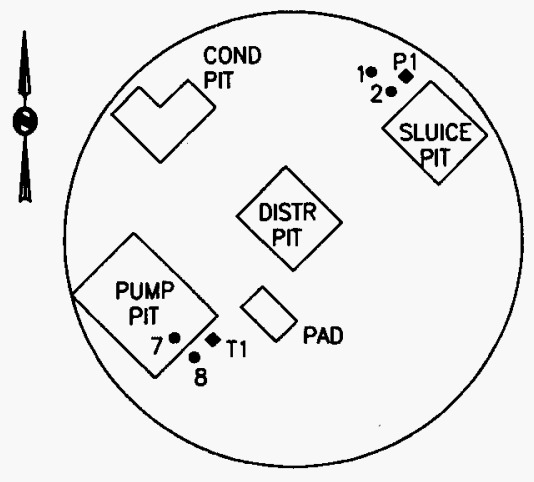

\begin{tabular}{|c|l|c|c|c|}
\hline \multirow{2}{*}{ RISER } & \multirow{2}{*}{ DESCRIPTION } & \multicolumn{2}{c|}{ RESISTANCE(OHMS) } & \multirow{2}{*}{ COMMENTS } \\
\cline { 3 - 4 } & & RISER & TOP & \\
\hline \hline 1 & $4 "$ BREATHER FLTR & 80.2 & 80.4 & \multirow{2}{*}{ U.G.R. } \\
\hline 2 & $12^{\prime \prime}$ OBSV PORT & 2.81 & 3.46 & \\
\hline 7 & $12^{\prime \prime}$ LIQ LEV REEL & 3.0 & $3.0(P)$ & PEDESTAL \\
\hline 8 & $4 "$ TEMP PROBE & 2.97 & 2.97 & \\
\hline P1 & PIPE & & 18.8 & \\
\hline T1 & INSTR TUNING & & 79.6 & \\
\hline & & & & \\
\hline & & & & \\
\hline & & & & \\
\hline & & & & \\
\hline & & & & \\
\hline & & & & \\
\hline & & & & \\
\hline & & & & \\
\hline
\end{tabular}

TEST EQUIP \# 785-77-47-001 CALIB DUE DATE $3-12-97$ 


\section{RISER RESISTANCE DATA SHEET}

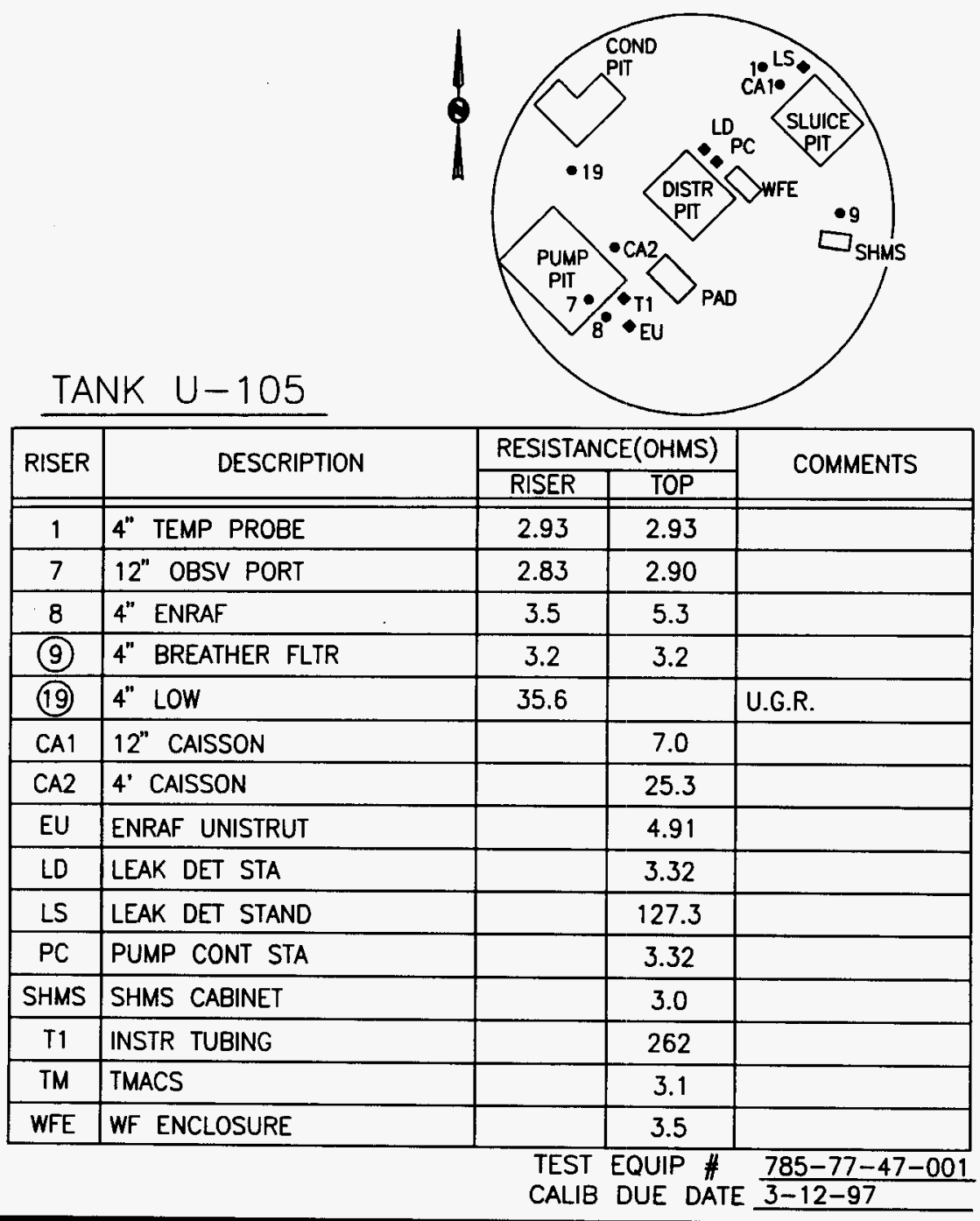




\section{RISER RESISTANCE DATA SHEET}

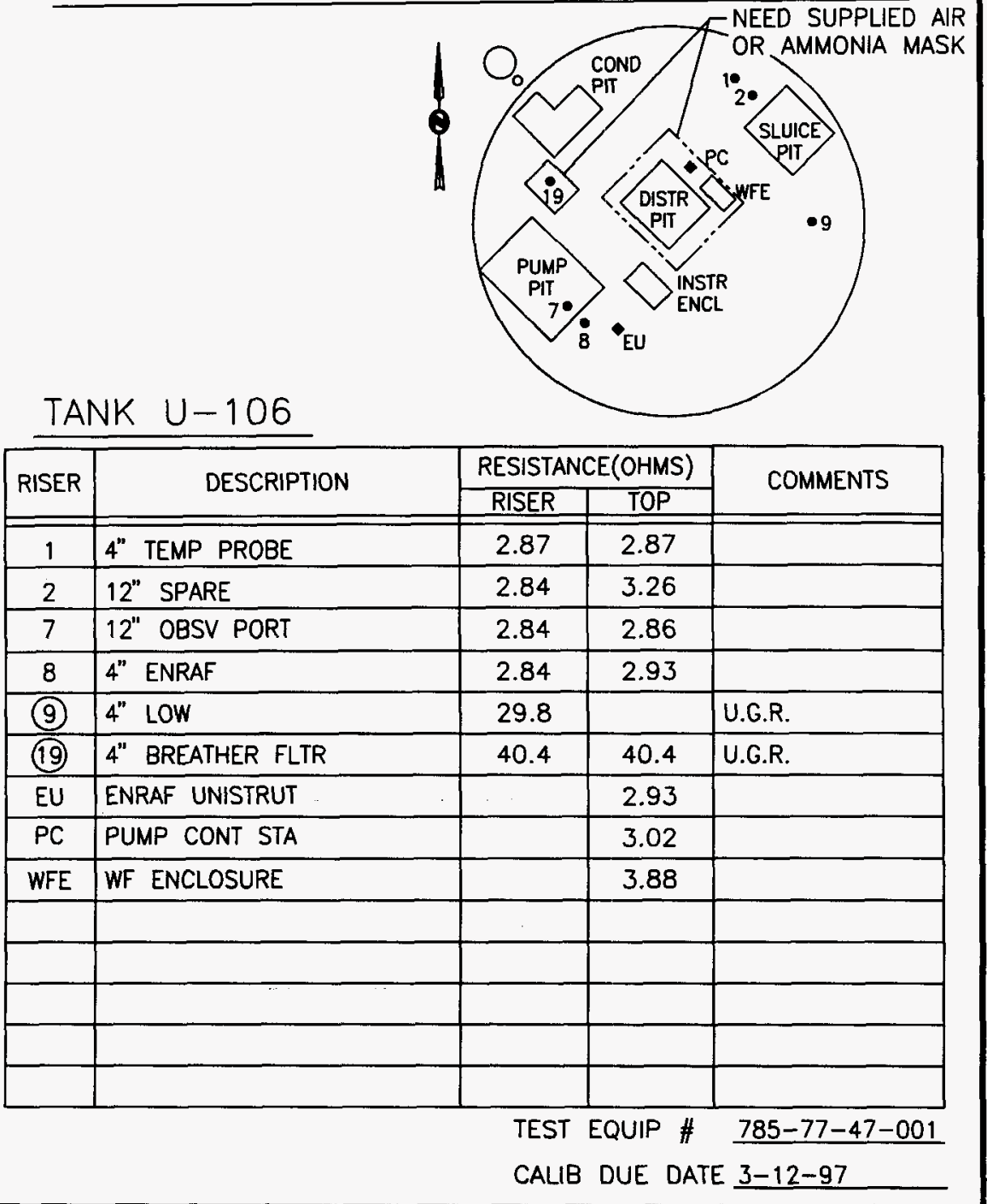


WHC-SD-WM-TR-034, REV. 0

\section{RISER RESISTANCE DATA SHEET}

TANK U-107

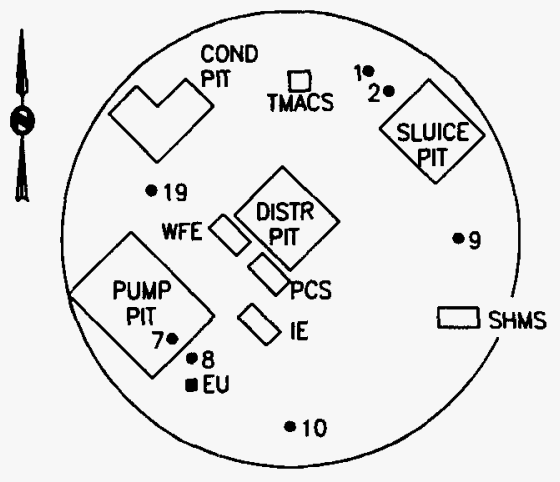

\begin{tabular}{|c|l|c|c|c|}
\hline \multirow{2}{*}{ RISER } & \multicolumn{1}{|c|}{ DESCRIPTION } & \multicolumn{2}{c|}{ RESISTANCE(OHMS) } & \multirow{2}{*}{ COMMENTS } \\
\cline { 3 - 4 } & & RISER & TOP & \\
\hline \hline TMACS & TMACS & & 3.09 & \\
\hline 1 & $4^{\prime \prime}$ TEMP PROBE & 2.84 & 2.84 & \\
\hline 2 & $12^{\prime \prime}$ SPARE & 2.81 & 2.84 & \\
\hline 7 & $12^{\prime \prime}$ SPARE & 2.83 & 2.87 & \\
\hline 8 & $4^{\prime \prime}$ ENRAF & 2.89 & 2.94 & \\
\hline$(9)$ & $4^{\prime \prime}$ FLANGE & 39.9 & 40.0 & U.G.R. \\
\hline (10) & $4^{\prime \prime}$ BREATHER FLTR & 3.10 & 3.12 & \\
\hline (19) & $4^{n}$ LOW & 41.8 & & U.G.R. \\
\hline EU & ENRAF UNISTRUT & & 2.96 & \\
\hline PCS & PUMP CONT STA & & 3.66 & \\
\hline SHMS & SHMS CABINET & & 3.04 & \\
\hline WFE & WF ENCLOSURE & & 3.62 & \\
\hline & & & & \\
\hline & & & & \\
\hline
\end{tabular}

TEST EQUIP \# 785-77-47-001

CALIB DUE DATE 3-12-97 


\section{RISER RESISTANCE DATA SHEET}

TANK U-108
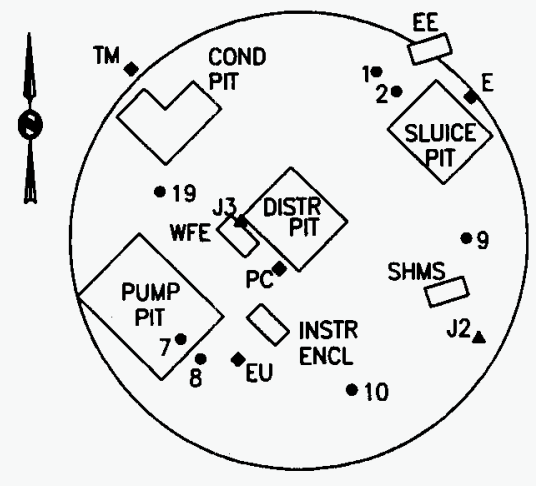

\begin{tabular}{|c|l|c|c|c|}
\hline \multirow{2}{*}{ RISER } & \multirow{2}{*}{ DESCRIPTION } & \multicolumn{2}{c|}{ RESISTANCE(OHMS) } & \multirow{2}{*}{ COMMENTS } \\
\cline { 3 - 4 } & & RISER & TOP & \\
\hline \hline 1 & $4^{\prime \prime}$ THERMO PROBE & 2.81 & 2.82 & \\
\hline 2 & $12^{\prime \prime}$ SPARE & 2.82 & 2.82 & \\
\hline 7 & $12^{\prime \prime}$ OBSV PORT & 2.81 & 2.81 & \\
\hline 8 & $4^{\prime \prime}$ ENRAF & 2.81 & 3.06 & \\
\hline$(9)$ & $4^{\prime \prime}$ SLUDGE MEAS & 37.0 & 37.1 & U.G.R. \\
\hline$(10)$ & $4^{\prime \prime}$ BREATHER FLTR & 3.01 & 3.01 & \\
\hline (19) & $4^{\prime \prime}$ LOW & 35.8 & & U.G.R. \\
\hline E & UNISTRUT ELEC OUT & & 3.77 & \\
\hline EE & ELEC EQPT 8'X2' & & 2.91 & \\
\hline EU & ENRAF UNISTRUT & & 3.05 & \\
\hline J1 & 2 JBOXES & & 3.49 & \\
\hline J2 & 2 JBOXES & & 2.99 & \\
\hline PC & PUMP CONT STA & & 3.77 & \\
\hline SHMS & SHMS CABINET & & 2.91 & \\
\hline TM & TMACS & & 2.88 & \\
\hline WFE & WF ENCLOSURE & & 3.88 & \\
\hline & & & & \\
\hline & & & & \\
\hline
\end{tabular}

TEST EQUIP \# 785-77-47-001

CALIB DUE DATE 3-12-97 


\section{RISER RESISTANCE DATA SHEET}

TANK U-109

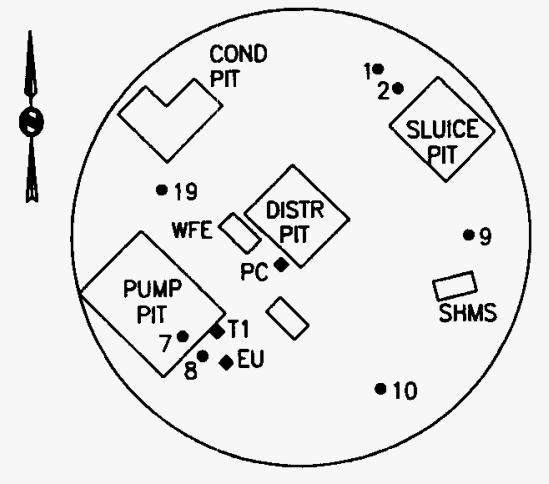

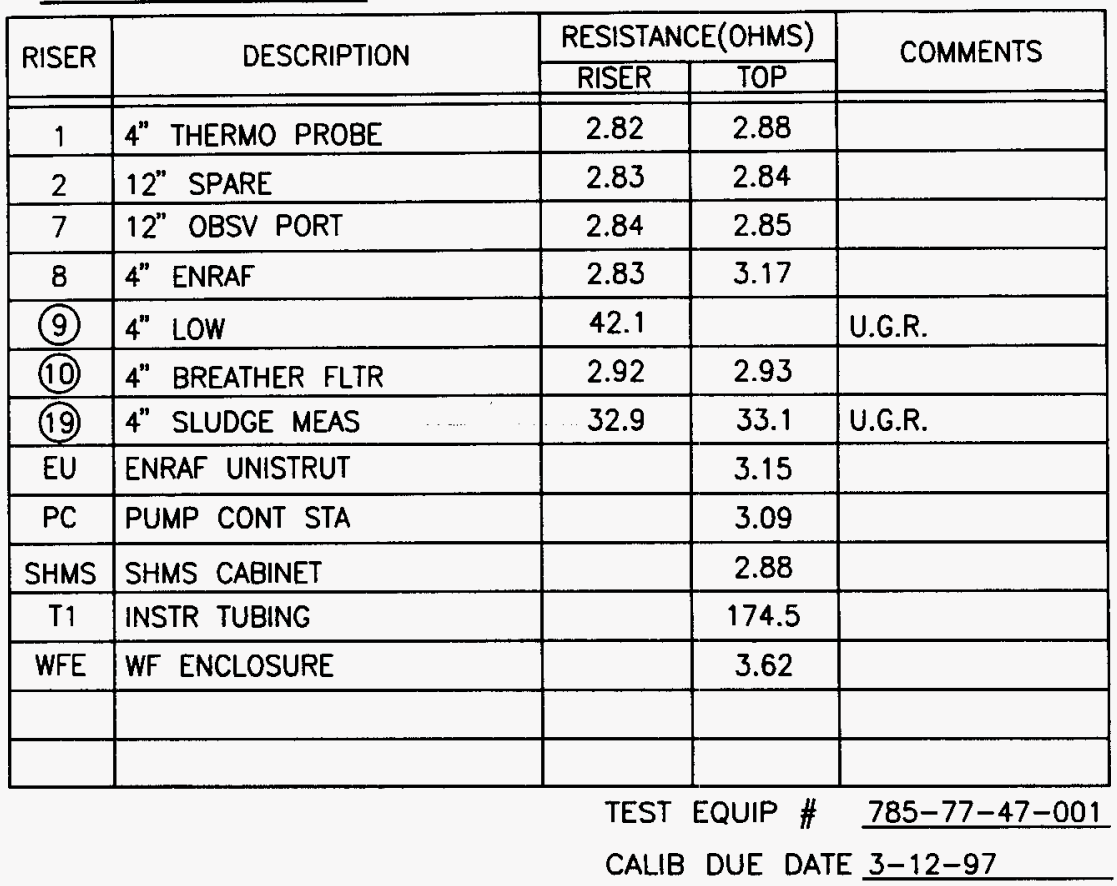




\section{RISER RESISTANCE DATA SHEET}

TANK U-110

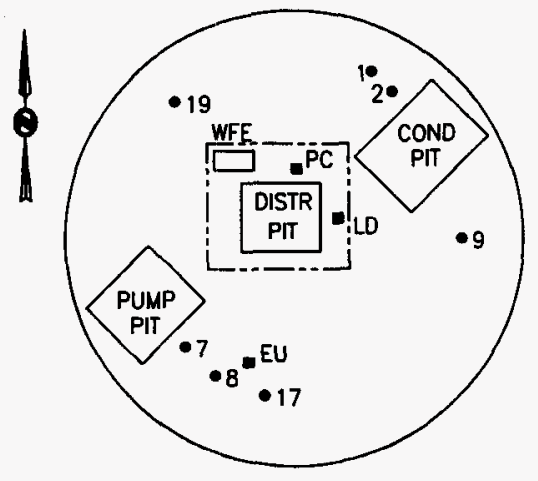

\begin{tabular}{|c|l|c|c|c|}
\hline \multirow{2}{*}{ RISER } & \multicolumn{1}{|c|}{ DESCRIPTION } & \multicolumn{2}{c|}{ RESISTANCE(OHMS) } & \multirow{2}{*}{ COMMENTS } \\
\cline { 3 - 4 } & & RISER & TOP & \\
\hline 1 & $4 "$ THERMO PROBE & 3.05 & 3.09 & \\
\hline 2 & $12^{\prime \prime}$ OBSV PORT & 3.05 & 3.05 & \\
\hline 7 & $12^{\prime \prime}$ SPARE & 3.2 & 67.9 & \\
\hline 8 & $4 "$ ENRAF & 3.2 & 3.5 & \\
\hline$(9)$ & $4 "$ BREATHER FLTR & 33.1 & 33.1 & U.G.R. \\
\hline (17) & $4 "$ SPARE & 18.8 & 18.8 & U.G.R. \\
\hline (19) & $4 "$ SLUDGE MEAS & 29.1 & 29.1 & U.G.R. \\
\hline EU & ENRAF UNISTRUT & & 3.6 & \\
\hline LD & LEAK DET STA & & 4.23 & \\
\hline PC & PUMP CONT STA & & 31.2 & \\
\hline WF & WF ENCLOSURE & & 3.72 & \\
\hline & & & & \\
\hline & & & & \\
\hline & & TEST EQUIP \# & DUE DATE 3-12-97 \\
\hline
\end{tabular}




\section{RISER RESISTANCE DATA SHEET}

TANK U-111

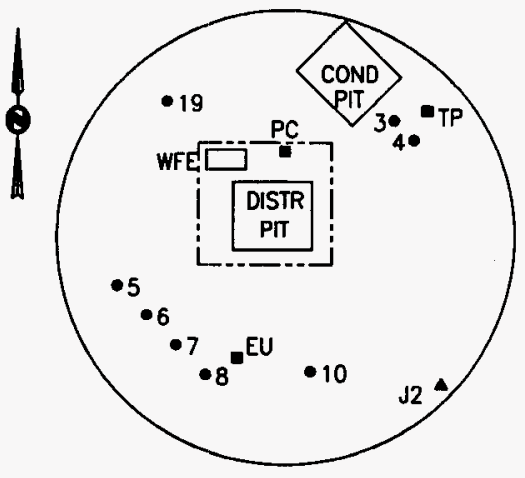

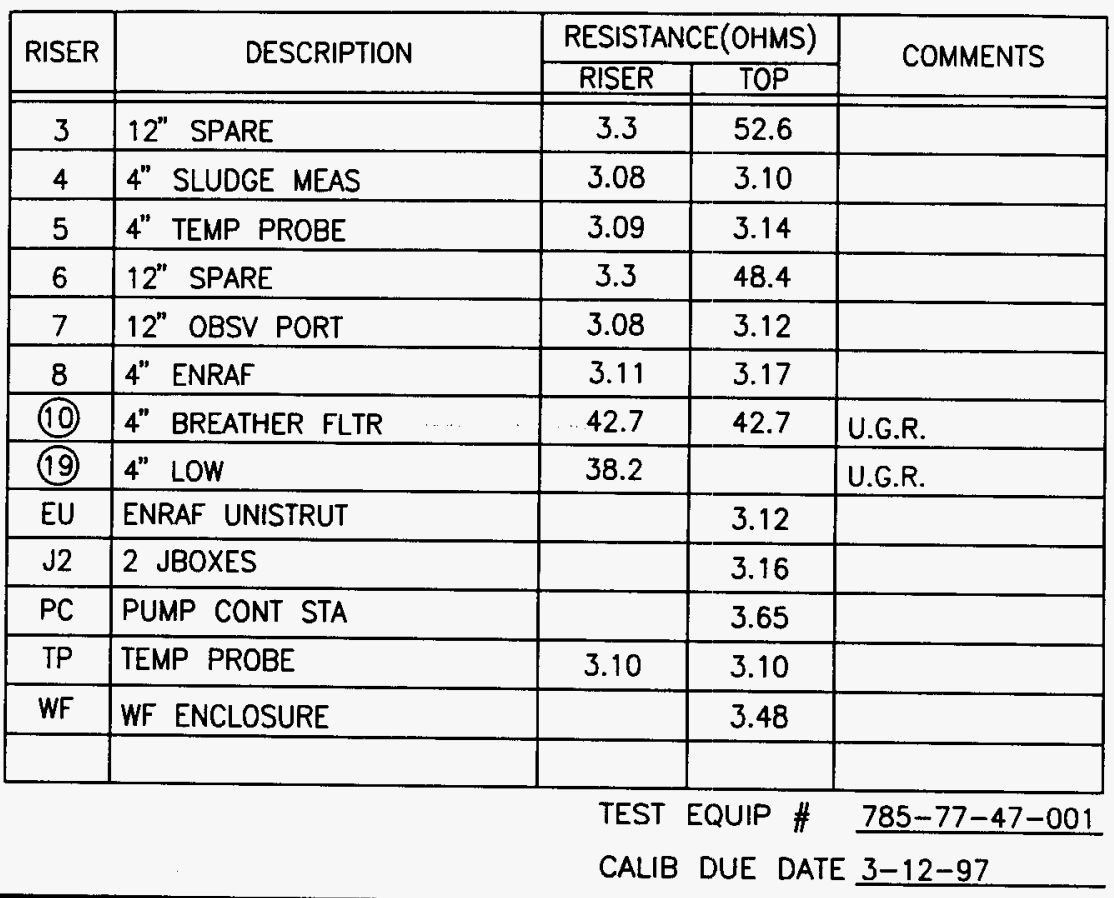




\section{RISER RESISTANCE DATA SHEET}

\begin{tabular}{|c|c|c|c|c|}
\hline \multirow{2}{*}{ RISER } & \multirow{2}{*}{ DESCRIPTION } & \multicolumn{2}{|c|}{ RESISTANCE(OHMS) } & \multirow{2}{*}{ COMMENTS } \\
\hline & & RISER & TOP & \\
\hline 1 & 4" BREATHER FLTR & 3.02 & 3.18 & \\
\hline 2 & 12" DIP TUBES & 3.03 & 5.28 & \\
\hline 3 & $12^{\prime \prime}$ SPARE & 3.09 & 7.10 & \\
\hline 5 & 4" THERMO PROBE & 3.03 & 2.99 & G.T.W. \\
\hline 6 & 12" SPARE & 3.02 & 65 & \\
\hline 7 & $12^{\prime \prime}$ OBSV PORT & 3.03 & 3.03 & \\
\hline 8 & 4" REEL & 3.03 & OPEN & \\
\hline$T M$ & TMACS & & 3.06 & \\
\hline & & & & \\
\hline & & & & \\
\hline & & & & \\
\hline & & & & \\
\hline & & & & \\
\hline & & & & \\
\hline & & TES & UUIP \# & $785-77-47-001$ \\
\hline & & $\mathrm{CAL}$ & UE DAT & $3-12-97$ \\
\hline
\end{tabular}


WHC-SD-WM-TR-034, REV. O

\section{RISER RESISTANCE DATA SHEET}

TANK U-201
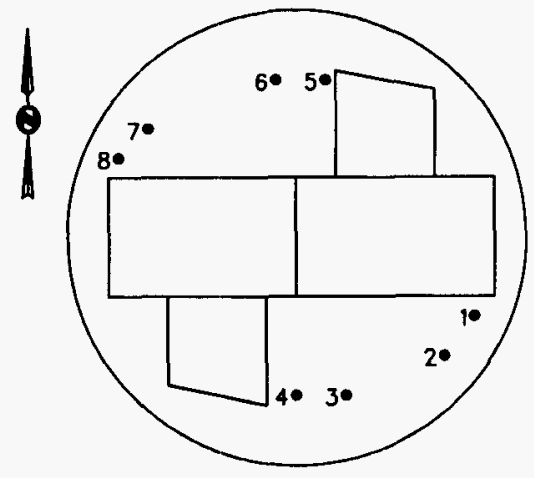

\begin{tabular}{|c|l|c|c|l|}
\hline \multirow{2}{*}{ RISER } & \multirow{2}{*}{ DESCRIPTION } & \multicolumn{2}{c|}{ RESISTANCE(OHMS) } & \multirow{2}{*}{ COMMENTS } \\
\cline { 3 - 4 } & & RISER & TOP & \\
\hline \hline 1 & $4^{\prime \prime}$ REEL & 46.0 & OPEN & \multirow{2}{*}{ U.G.R. } \\
\hline 2 & $12^{\prime \prime} 3^{\prime \prime}$ SM/4"SPARE & 3.13 & 3.13 & \\
\hline 3 & $12^{\prime \prime}$ DIP TUBES & 3.18 & 3.20 & \\
\hline 4 & $4^{\prime \prime}$ THERMO TREE & 3.18 & 3.04 & G.T.W. \\
\hline 5 & $4 "$ THERMOWELL & & 480 & RISER ENCASED \\
\hline 6 & $12^{\prime \prime}$ OBSV PORT & 26.3 & 26.3 & U.G.R. \\
\hline 7 & $12^{\prime \prime}$ DIP TUBES & 27.9 & 27.9 & U.G.R. \\
\hline 8 & $4 "$ BREATHER FLTR & 48.5 & 48.6 & U.G.R. \\
\hline & & & & \\
\hline & & & & \\
\hline & & & & \\
\hline & & & & \\
\hline & & & & \\
\hline & & & & \\
\hline
\end{tabular}

TEST EQUIP \# 785-77-47-001

CALIB DUE DATE 3-12-97 


\section{RISER RESISTANCE DATA SHEET}

TANK U-202

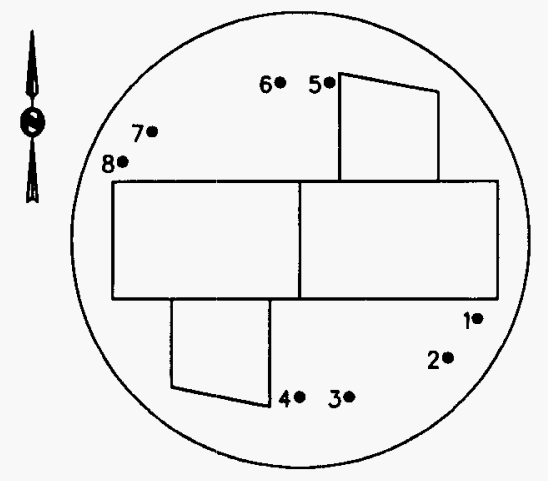

\begin{tabular}{|c|c|c|c|c|}
\hline \multirow{2}{*}{ RISER } & \multirow{2}{*}{ DESCRIPTION } & \multicolumn{2}{|c|}{ RESISTANCE(OHMS) } & \multirow{2}{*}{ COMMENTS } \\
\hline & & RISER & TOP & \\
\hline 1 & 4" REEL & 46.4 & OPEN & U.G.R. \\
\hline 2 & 12" $3^{\prime \prime}$ SM/4" SPARE & 36.2 & 36.4 & U.G.R. \\
\hline 3 & $12^{\prime \prime}$ DIP TUBES & 34.7 & 437 & U.G.R. \\
\hline 4 & 4" THERMO TREE & 4.07 & 4.58 & \\
\hline 5 & 4" THERMOWELL & & 474 & RISER ENCASED \\
\hline 6 & 12" OBSV PORT & 3.62 & 3.62 & \\
\hline 7 & $12^{\prime \prime}$ DIP TUBES & 31.6 & 31.7 & U.G.R. \\
\hline 8 & $4^{\prime \prime}$ BREATHER FLTR & 3.69 & $1.19 \mathrm{~K}$ & \\
\hline & & & & \\
\hline & & & & \\
\hline & & & & \\
\hline & & & & \\
\hline & & & & \\
\hline & & & & \\
\hline & & $\begin{array}{l}\text { TES } \\
\text { CAL }\end{array}$ & $\begin{array}{l}\text { QUIP \# } \\
\text { DUE DA }\end{array}$ & $\begin{array}{l}785-77-47-001 \\
3-12-97\end{array}$ \\
\hline
\end{tabular}




\section{RISER RESISTANCE DATA SHEET}

TANK U-203

\begin{tabular}{|c|c|c|c|c|}
\hline \multirow{2}{*}{ RISER } & \multirow{2}{*}{ DESCRIPTION } & \multicolumn{2}{|c|}{ RESISTANCE(OHMS) } & \multirow{2}{*}{ COMMENTS } \\
\hline & & RISER & TOP & \\
\hline 1 & 4" REEL & 52.3 & OPEN & U.G.R. \\
\hline 2 & 12" $3 "$ SM/4" SPARE & 3.67 & 4.82 & \\
\hline 3 & 12" DIP TUBES & 3.76 & 6.34 & \\
\hline 4 & 4" THERMO TREE & 3.83 & 3.80 & G.T.W. \\
\hline 5 & 4" THERMOWELL & & 474 & RISER ENCASED \\
\hline 6 & 12" OBSV PORT & 27.4 & 27.4 & U.G.R. \\
\hline 7 & 12" DIP TUBES & 22.3 & 22.3 & U.G.R. \\
\hline 8 & 4" BREATHER FLTR & 22.3 & 22.3 & U.G.R. \\
\hline & & & & \\
\hline & & & & \\
\hline & & & & \\
\hline & & & & \\
\hline & & & & \\
\hline & & & & \\
\hline & & $\begin{array}{l}\text { TEST } \\
\text { CALI }\end{array}$ & $\begin{array}{l}\text { QUIP \# } \\
\text { DUE DA }\end{array}$ & $\begin{array}{l}785-77-47-001 \\
3-12-97\end{array}$ \\
\hline
\end{tabular}




\section{RISER RESISTANCE DATA SHEET}

TANK U-204

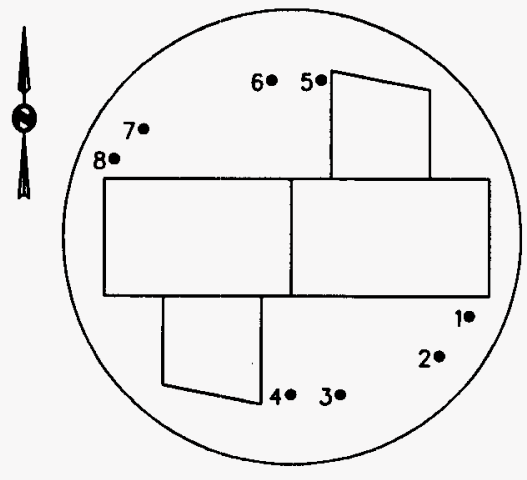

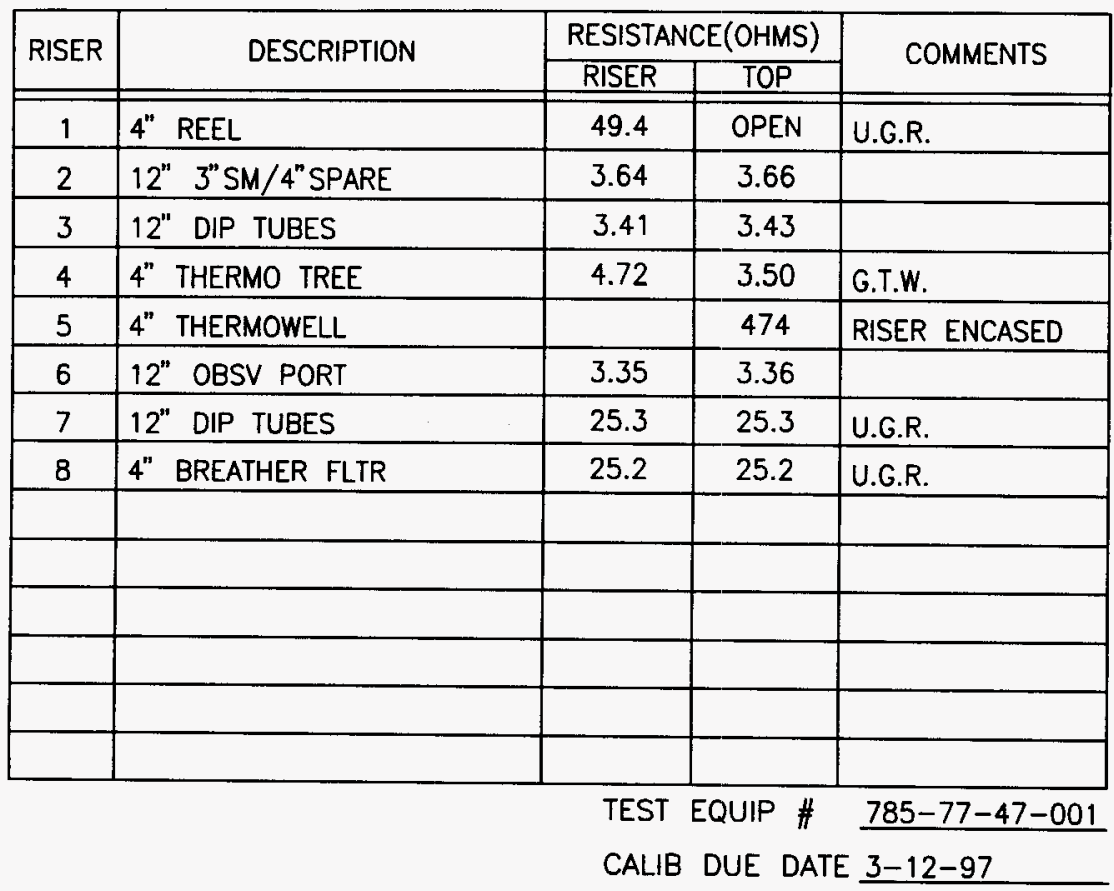




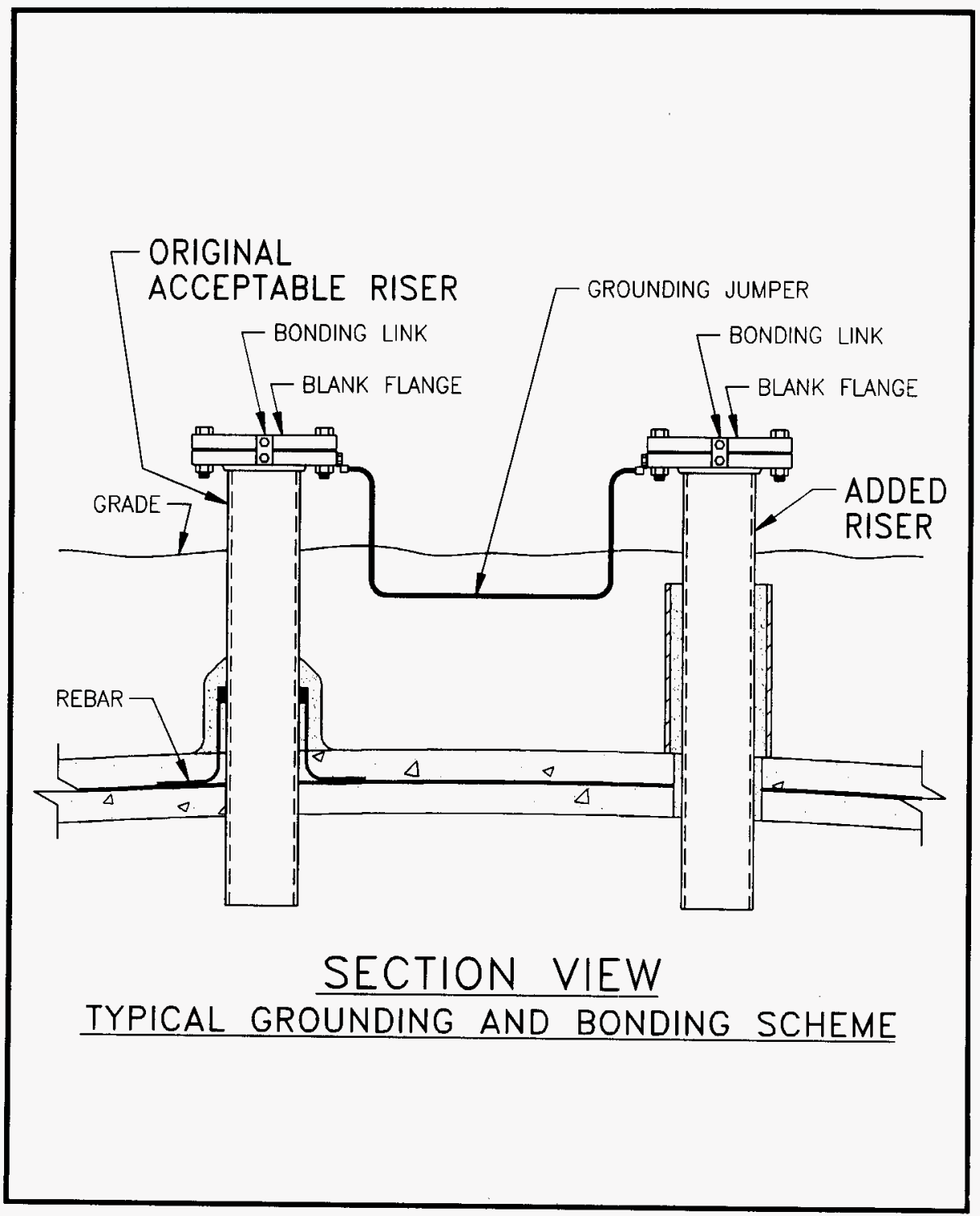

$D-1$ 


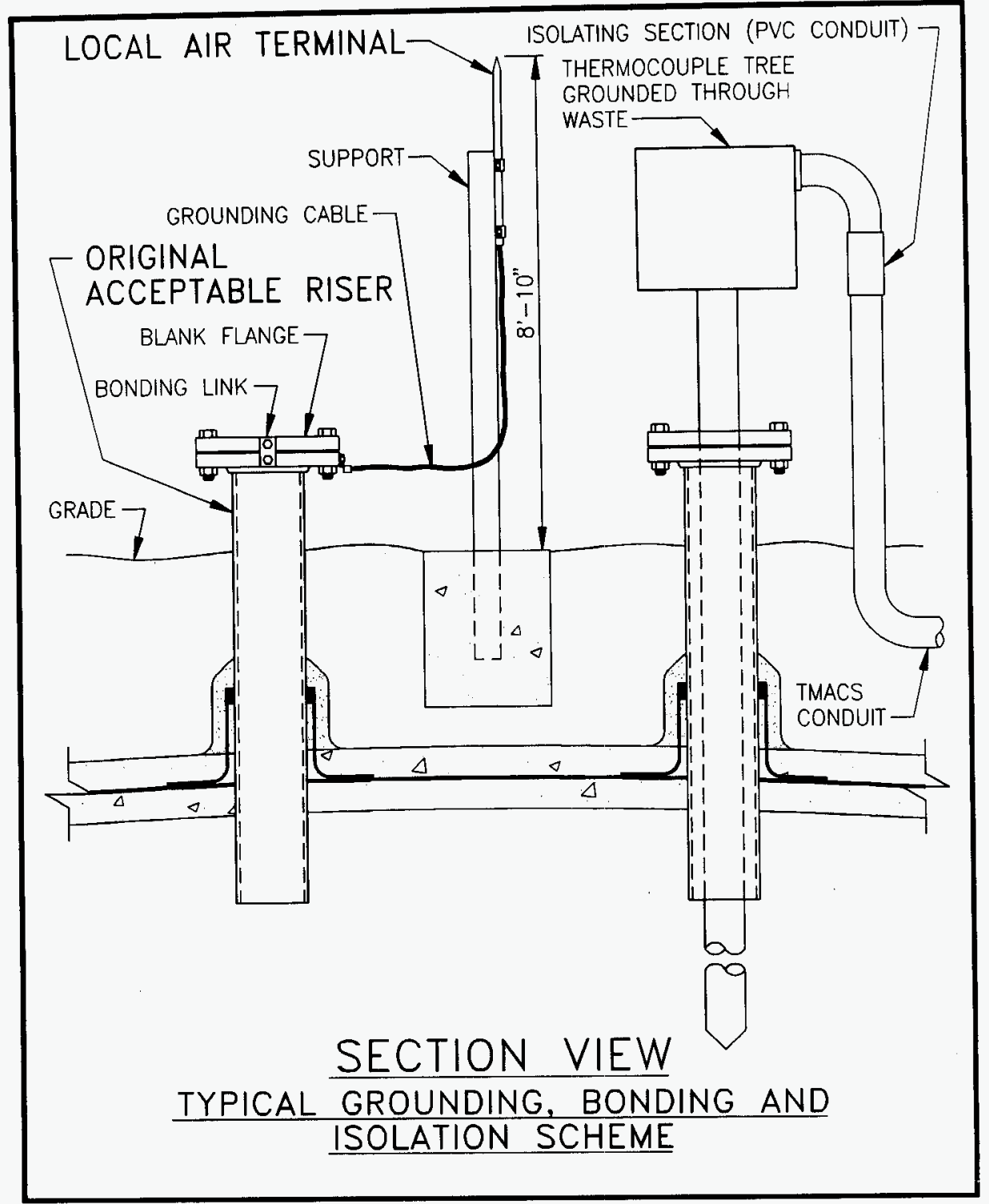




\section{DISTRIBUTION SHEET}

\begin{tabular}{|c|c|c|c|c|c|}
\hline \multirow{2}{*}{$\begin{array}{l}\text { To } \\
\text { Distribution }\end{array}$} & \multirow{2}{*}{\multicolumn{3}{|c|}{$\begin{array}{l}\text { From } \\
\text { Plant Controls } 74 E 41\end{array}$}} & \multirow{2}{*}{\multicolumn{2}{|c|}{ Page 1 of 1}} \\
\hline & & & & & \\
\hline \multirow{2}{*}{\multicolumn{4}{|c|}{$\begin{array}{l}\text { Project Title/Work Order } \\
\text { Lightning Hazard }\end{array}$}} & \multicolumn{2}{|c|}{ EDT No. 614427} \\
\hline & & & & \multicolumn{2}{|l|}{ ECN No. } \\
\hline Name & MSIN & $\begin{array}{l}\text { Text } \\
\text { With All } \\
\text { Attach. }\end{array}$ & Text Only & $\begin{array}{l}\text { Attach./ } \\
\text { Appendix } \\
\text { Only }\end{array}$ & $\begin{array}{c}\text { EDT/ECN } \\
\text { Only }\end{array}$ \\
\hline L. Kiewert & & $52-02$ & $\bar{x}$ & & \\
\hline W. L. Cowley & & A3-37 & $x$ & & \\
\hline P. F. Kison & & T4-07 & $x$ & & \\
\hline V. P. Ocampo & & G3-17 & $x$ & & \\
\hline J. G. Propson & & $\mathrm{R} 2-36$ & $x$ & & \\
\hline D. M. Stenkamp & & $S 2-02$ & $x$ & & \\
\hline R. A. Huckfeldt & & R3-01 & $x$ & & \\
\hline J. E. Wells & & S5-05 & $x$ & & \\
\hline J. J. Zach & & S7-14 & $x$ & & \\
\hline S. H. Rifaey & & $R 1-56$ & $x$ & & \\
\hline W. W. Jenkins & & $S 2-24$ & $x$ & & \\
\hline D. A. Bragg & & $S 5-05$ & $x$ & & \\
\hline G. T. Frater & & $\$ 7-16$ & $x$ & & \\
\hline
\end{tabular}

